\title{
Cardiovascular drugs and the risk of systemic autoimmune diseases : pharmacoepidemiological and experimental approaches
}

Citation for published version (APA):

de Jong, H. J. I. (2014). Cardiovascular drugs and the risk of systemic autoimmune diseases : pharmacoepidemiological and experimental approaches. [Doctoral Thesis, Maastricht University]. Maastricht University. https://doi.org/10.26481/dis.20141218hj

Document status and date:

Published: 01/01/2014

DOI:

10.26481/dis.20141218hj

Document Version:

Publisher's PDF, also known as Version of record

Please check the document version of this publication:

- A submitted manuscript is the version of the article upon submission and before peer-review. There can be important differences between the submitted version and the official published version of record.

People interested in the research are advised to contact the author for the final version of the publication, or visit the DOI to the publisher's website.

- The final author version and the galley proof are versions of the publication after peer review.

- The final published version features the final layout of the paper including the volume, issue and page numbers.

Link to publication

\footnotetext{
General rights rights.

- You may freely distribute the URL identifying the publication in the public portal. please follow below link for the End User Agreement:

www.umlib.nl/taverne-license

Take down policy

If you believe that this document breaches copyright please contact us at:

repository@maastrichtuniversity.nl

providing details and we will investigate your claim.
}

Copyright and moral rights for the publications made accessible in the public portal are retained by the authors and/or other copyright owners and it is a condition of accessing publications that users recognise and abide by the legal requirements associated with these

- Users may download and print one copy of any publication from the public portal for the purpose of private study or research.

- You may not further distribute the material or use it for any profit-making activity or commercial gain

If the publication is distributed under the terms of Article 25fa of the Dutch Copyright Act, indicated by the "Taverne" license above, 


\section{ISBN}

978-94-6259-480-7

\section{Design and lay-out}

Promotie In Zicht, Arnhem

\section{Print}

Ipskamp Drukkers, Enschede

(C) H.J.I. de Jong 2014

All rights reserved. No part of this book may be reproduced in any form or by any means without permission of the author.

Financial support by the Dutch Arthritis Association (Reumafonds), the Royal Dutch Pharmacists Association (KNMP), the Dutch Heart Foundation (Nederlandse Hartstichting), Netherlands Side Affects Fund (Nederlandse Bijwerkingen Fonds) and the National Institute for Public Health and the Environment (RIVM) for the publication of this thesis are gratefully acknowledged. 


\section{Cardiovascular drugs and the risk of systemic autoimmune diseases \\ Pharmacoepidemiological and experimental approaches}

\section{Proefschrift}

ter verkrijging van de graad van doctor aan de Universiteit Maastricht, op gezag van de Rector Magnificus, Prof. Dr. L.L.G. Soete, volgens het besluit van het College van Decanen,

in het openbaar te verdedigen op donderdag 18 december 2014

om 16.00 uur

door

Hielkje Johanna Ida de Jong

Geboren te Dronten

op 13 juli 1976 


\section{Promotores}

Prof. dr. H. van Loveren

Prof. dr. J.W. Cohen Tervaert

\section{Copromotores}

Dr. R.J. Vandebriel

Dr. O.H. Klungel

\section{Beoordelingscommissie}

Prof. dr. H. ten Cate (voorzitter)

Prof. dr. W. van den Berg (Radboud Universiteit Nijmegen)

Prof. dr.A.C.G. Egberts (Universitair Medisch Centrum Utrecht)

Prof. dr. J. Kleinjans

Prof. dr. P.W. de Leeuw

Funding for this research project was provided by the National Institute for Public Health and the Environment (RIVM) (Project S340040). 


\section{CONTENTS}

$\begin{array}{ll}\text { Abbreviations } & 6\end{array}$

$\begin{array}{lll}\text { Chapter } 1 \text { General introduction } & 9\end{array}$

Chapter 2 Signal detection for statin-assocated autoimmune disorders in $\quad 35$ spontaneous reporting databases

2.1 Association between statin use and lupus-like syndrome $\quad 37$ using spontaneous reports

2.2 Statin-associated polymyalgia rheumatica. An analysis using WHO Global Individual Case Safety Database: A case/non-case approach

2.3 Giant cell arteritis and polymyalgia rheumatica after re-exposure to a statin

Chapter 3 Testing the association between cardiovascular drug use and the incidence of autoimmune diseases in electronic health record databases

3.1 Use of statins is associated with an increased risk of rheumatoid arthritis

3.2 Pattern of risks of rheumatoid arthritis among patients using statins: a cohort study with the clinical practice research datalink

3.3 Angiotensin-converting enzyme inhibitors or angiotensin II receptor blockers and the risk of developing rheumatoid arthritis in antihypertensive drug users

Chapter 4 Statin use and autoimmunity

4.1 Statin use and markers of immunity in the Doetinchem cohort study

Chapter 5 Causal relationship statin use and autoimmune diseases

5.1 Statins accelerate the onset of collagen type II-induced arthritis in mice

$\begin{array}{lll}\text { Chapter } 6 & \text { Summary and general discussion } & 187\end{array}$

$\begin{array}{lll}\text { Chapter } 7 & \text { Valorisation Addendum } & 225\end{array}$

$\begin{array}{lll}\text { Chapter } 8 & \text { Dutch summary | Nederlandse samenvatting } 237\end{array}$

Acknowledgements | Dankwoord 249

List of Publications 255

List of Co-authors 258

Curriculum vitae 261 


\section{ABBREVIATIONS}

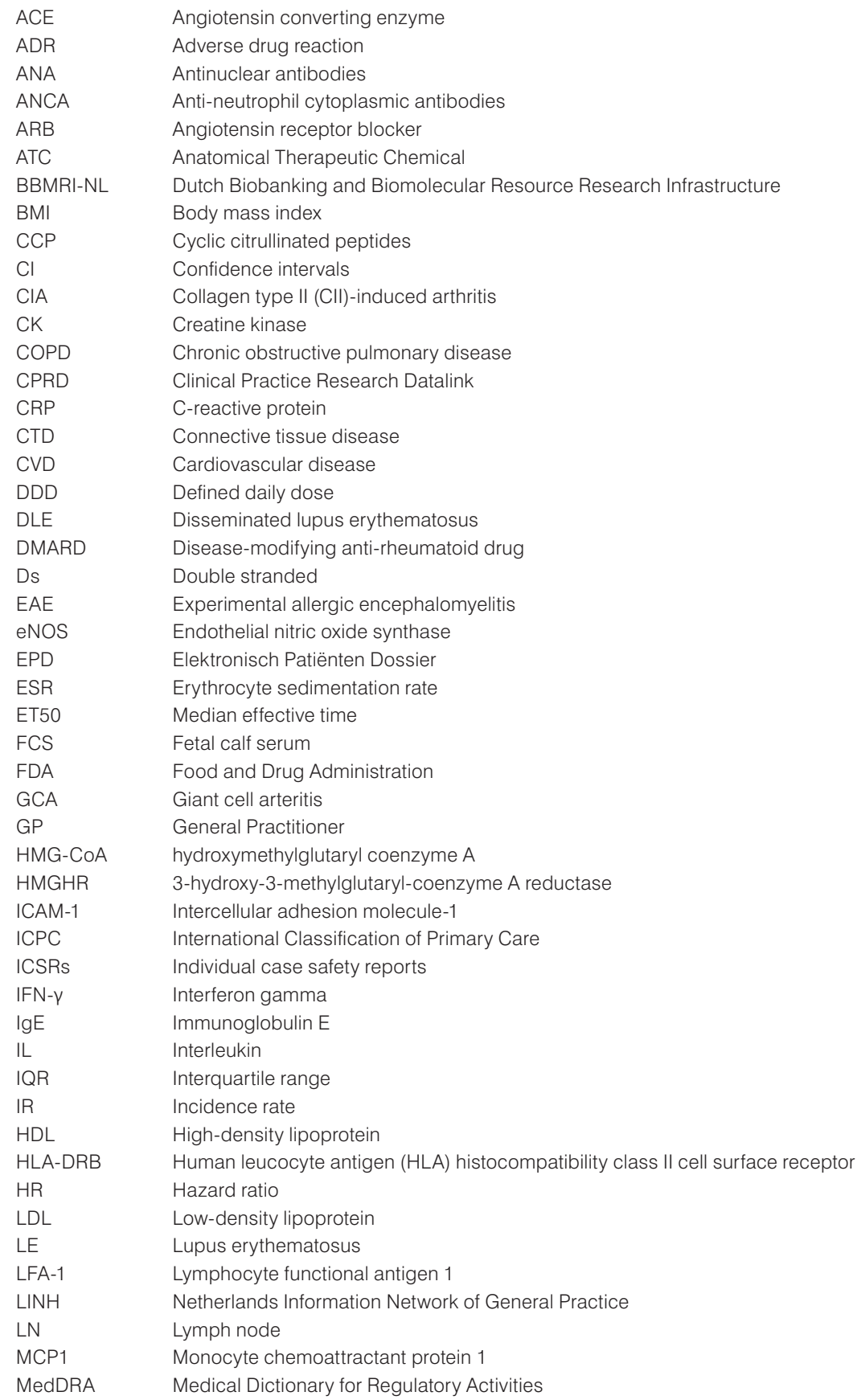




$\begin{array}{ll}\text { MHC } & \text { Major histocompatibility complex } \\ \text { MPO } & \text { Myeloperoxidase } \\ \text { MS } & \text { Multiple sclerosis } \\ \text { NET } & \text { Neutrophil extracellular traps } \\ \text { NSAID } & \text { Non-steroidal anti-inflammatory drug } \\ \text { OR } & \text { Odds ratio } \\ \text { PBS } & \text { Phosphate buffered saline } \\ \text { PHARMO-RLS } & \text { PHARMO-Record Linkage System } \\ \text { PMR } & \text { Polymyalgia rheumatica } \\ \text { PPI } & \text { Proton pump inhibitors } \\ \text { RA } & \text { Rheumatoid arthritis } \\ \text { RCT } & \text { Randomised controlled trial } \\ \text { RF } & \text { Rheumatoid factor } \\ \text { ROR } & \text { Reporting odds ratio } \\ \text { SCORE } & \text { Systematic Coronary Risk Evaluation Project } \\ \text { SD } & \text { Standard deviations } \\ \text { SLE } & \text { Systemische lupus erythematosus } \\ \text { TGF- } \beta & \text { Transforming factpr beta } \\ \text { Th } & \text { Thelper } \\ \text { TIA } & \text { Transient ischaemic attack } \\ \text { TNF-a } & \text { Tumor necrosis factor alpha } \\ \text { UK } & \text { United Kingdom } \\ \text { WHO-ART } & \text { World Health Organisation Adverse Reaction Terminology } \\ \text { WHO UMC } & \text { World Health Organisation Uppsala Monitoring Centre } \\ & \end{array}$




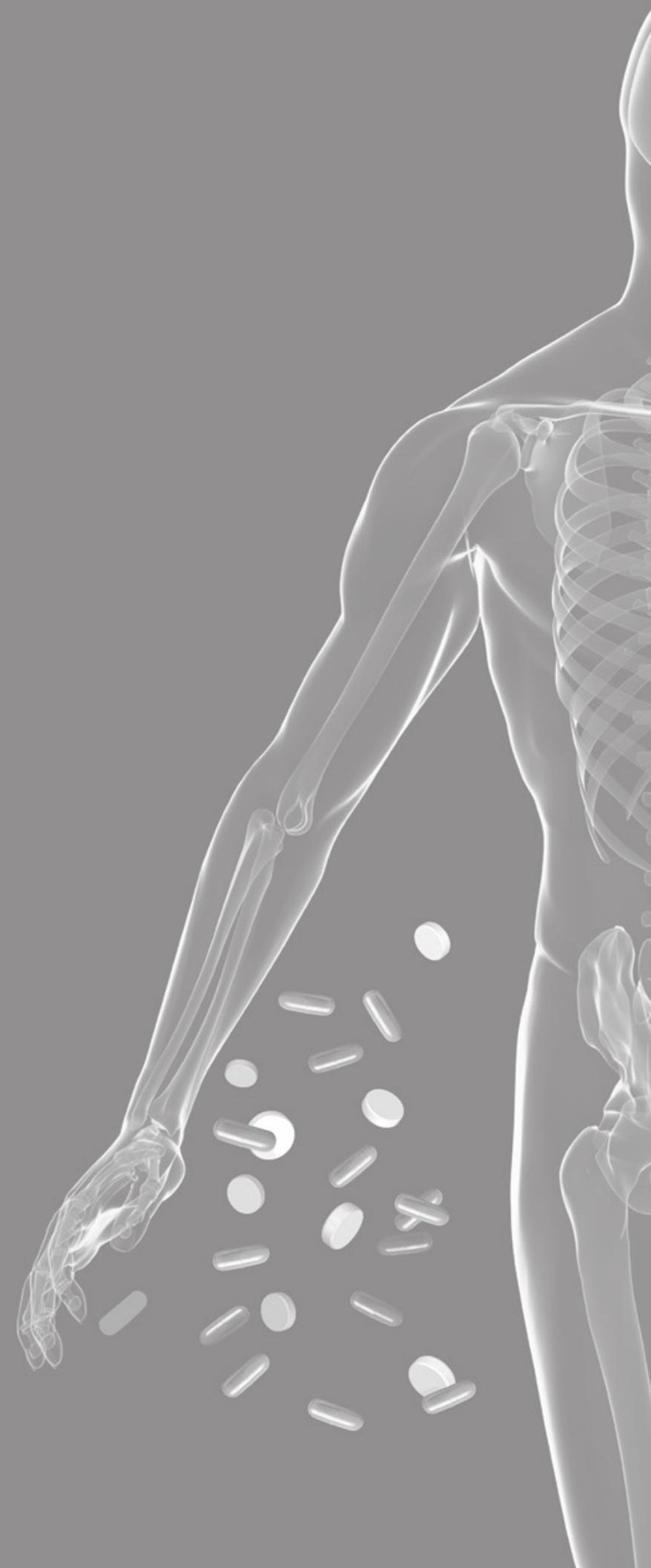





\section{INTRODUCTION}

Cardiovascular disease (CVD) is the most common cause of death in the Netherlands, accounting for approximately 39,642 deaths each year 1 . Worldwide, the number of deaths from cardiovascular disease was estimated at 17.3 million in 2008, and it is expected to increase to approximately 23.6 million patients by 20302 . Treatment with aspirin, statins and anti-hypertensive agents is currently being recommended for primary and secondary prevention of cardiovascular diseases ${ }^{3}$. Besides their lipid and blood pressure lowering effects, emerging evidence indicates that statins and anti-hypertensive agents, specifically angiotensin converting enzyme (ACE) inhibitors and angiotensin receptor blockers (ARBs), could also be related to an improvement in endothelial dysfunction, anti-inflammatory and immunomodulatory effects ${ }^{4-23}$.

The immune system is a system of biological structures and processes, intended to protect the body from potentially harmful substances by recognising and responding to a wide variety of agents, from viruses to tumour cells, and distinguish them from the body's own healthy tissue. When the immune system fails to distinguish between its own healthy tissue and these potential harmful substances, it results in erroneously attacks and destroys healthy body tissue, a condition also known as autoimmunity 24 . The development of autoimmunity is a multi-factorial process, including genetic, epigenetic and environmental factors ${ }^{25}$, and despite many years of extensive research, exact mechanisms responsible for the initiation of autoimmunity have not been fully elucidated. Several studies have demonstrated that environmental factors play a major role in this process of initiating autoimmunity 26-30 and may be responsible for the increased prevalence of autoimmune diseases in highly industrialised countries 31 . Environmental factors operating in a genetically susceptible individual may directly initiate, facilitate, or exacerbate pathological immune process, induce mutations in genes coding for immunoregulatory factors, or modify immune tolerance or regulatory and immune effector pathways ${ }^{31}$. One of the environmental factors that may be of importance in the development or progression of autoimmunity is exposure to certain drugs 32 .

\section{DRUGS AND AUTOIMMUNITY}

Adverse drug reactions may occur in approximately five to fifteen percent of therapeutic drug courses ${ }^{33,34}$, and in the United States of America (USA) yearly, more than 100 000 deaths were ascribed to serious adverse drug reactions ${ }^{34-36}$. The first cases in literature on drug-induced autoimmunity were patients developing various lupus-like symptoms after receiving sulphadiazine 37 and hydralazine 38,39. In 1953, approximately seven percent of the patients treated with hydralazine developed these lupus-like 
features, and similar percentages have been observed recently 32,40. In the last decade, there has been an increase in reports of drug-induced autoimmunity $41-43$. Numerous drugs have been reported to be associated with autoimmunity or resemble autoimmune disorders (table 1), and these numbers continue to increase as new therapeutics are introduced into practice 40,44 . There is an increasing number of immunomodulatory drugs on the market, however, only a small group of patients who were treated with these drugs over a prolonged period of time, develop a systemic drug-induced syndrome 45. For instance, only ten percent of systemic lupus erythematosus (SLE) patients in the USA are defined as drug-induced lupus, representing an estimation of 15000 to 30000 patients per year 40 .

Table 1 Drugs associated with lupus-like syndrome

\begin{tabular}{ll} 
ACE inhibitors & Captopril, Enalapril, Lisinopril, Cilazapril \\
\hline Antiarrthmics & Procainamide, Quinidine, Disopyramide, Propafenone, Amiodarone \\
\hline Antibiotics & Isoniazid, Minocycline, Nitrofurantoin \\
\hline Anticonvulsants & $\begin{array}{l}\text { Carbamazepine, Ethosuximide, Phenytoin, Primidone, } \\
\text { Trimethadione }\end{array}$ \\
\hline $\begin{array}{l}\text { Antihypertensive } \\
\text { agents }\end{array}$ & Hydralazine, Methyldopa, Clonidine, Minoxidil, Prazosin \\
\hline Anti-inflammatory & D-Penicillamine, Sulfasalazine, Phenylbutazone \\
\hline Antipsychotics & Chlorpromazine, Chlorprothixene, Lithium carbonate, Phenelzine \\
\hline Antithyroid & Propylthiouracil, Methimazole, Carbimazole \\
\hline Beta blockers & Acebutolol, Atenolol, Labetalol, Pindolol \\
\hline Biologicals & $\begin{array}{l}\text { Etanercept, Infliximab, Adalimumab, Ipilimumab (anti-CTLA-4), } \\
\text { IFN-a, IFN- } \beta \text {, IL-2 }\end{array}$ \\
\hline Diuretics & Hydrochlorothiazide, Chlorthalidone \\
\hline Statins & Atorvastatin, Fluvastatin, Lovastatin, Pravastatin, Simvastatin \\
\hline Adapted from Borchers et al. ${ }^{40,}$, Vasoo 45, Wiik 46, Rubin 50, Fadel et al.254
\end{tabular}

To date, no definite diagnostic criteria for drug-induced autoimmunity are available; however, there is a general consensus in setting this diagnosis. According to this general consensus, there should be a temporal relationship between continuous drug exposure (> one month) and clinical findings. The clinical findings should not be present in the patient prior to the use and should be resolved upon cessation of the offending drug 32,44-46. In addition, drug-induced autoimmunity was identified when 
patients showed similar symptoms after re-exposure to the drug ${ }^{32}$, or was accompanied by the presence of specific antibodies indicative of drug-induced autoimmunity, i.e., anti-histone or human neutrophil elastase anti-neutrophil cytoplasmic antibodies (HNE-ANCA) 47-49.

Several hypotheses for the mechanism underlying drug-induced autoimmunity have been postulated but none of them have been clearly demonstrated. Currently, available data strongly suggest that there is no single mechanism responsible for drug-induced autoimmunity. To date, four main mechanisms have been hypothesised in the literature 50,51 .

The first mechanism is based on the presumed capacity of either the drug or its metabolites to produce stable complexes with self-macromolecules or stimulate lymphocytes directly. Essentially, this concept is a drug hypersensitivity mechanism in which the drug or its reactive metabolites bind to the protein (hapten), making it 'foreign' and stimulating an immune response against the hapten or possibly self-antigens (molecular mimicry) 43,45,52,53. As suggested in several studies, drugs or their reactive metabolites are capable of inducing specific $T$ cell responses, apparently by the altered self-proteins, however, there is no convincing evidence that this results in autoimmune diseases 40,50 .

Secondly, it has been postulated that certain reactive drug metabolites directly cause cell death via a non-immune mediated process of direct cytotoxicity 45,52. To date, six drugs (i.e. hydralazine, procainamide, isoniazid, quinidine, propylthiouracil and chlorpromazine) have been associated with a relatively high incidence of drug-induced lupus-like syndrome. These drugs can develop into highly reactive metabolites by myeloperoxidase (MPO), an enzyme found in neutrophils and monocytes ${ }^{54}$. These reactive metabolites may cross-stimulate $T$ cells from healthy individuals 55 , stimulate Thelper (Th) cells to act on $B$ cells 56 , thereby enhancing, in the presence of interleukin (IL)-17, the production of MPO-ANCA, an autoantibody often associated with drug-induced lupus-like syndrome and drug-induced vasculitis 57-60

A second mechanism of the cytotoxicity of reactive metabolites involves apoptosis and NETosis. Vasoo postulated that these reactive metabolites alter degradation and clearance of apoptotic cells, which could lead to the loss of self-tolerance ${ }^{45}$. Recently, it has been demonstrated that these drugs, quinidine and procainamide, could indeed inhibit the uptake of apoptotic and necrotic cells by macrophages 61 , which may increase the autoantibody production against cellular antigens 62 . In addition, certain drugs including hydralazine, isoniazid, quinidine, or chlorpromazine, but not procainamide induce apoptosis of activated peripheral blood mononuclear cells 63 . Alternatively, ANCA can directly induce NETosis, a unique form of cell death other than apoptosis 64,65 . In contrast to apoptotic cells, NETotic cells do not display 'eat-me' signals, e.g., phosphatidylserine on the surface of apoptotic cells 66 , before plasma membrane disruption, preventing clearance of NETotic cells by phagocytosis 
64. During NETosis, neutrophil extracelllar traps (NETs) are released, which are assembled from nuclear chromatin, associated with histones, cytoplasmic and granular proteins, including the enzymes elastase and MPO 64,67. NETs trap and kill invading microbes extracellularly, even after the neutrophils are dead 65,67. However, several studies have demonstrated that impaired regulation of NETs could trigger an autoimmune response against components of NETs and induce autoimmune diseases 68-70. Recently, Nakazawa et al. showed that the anti-thyroid drug propylthiouracil impairs conformation and degradation of NETs, and triggers the production of MPO-ANCA antibodies, resulting in the development of MPO ANCA-associated vasculitis 71 .

The third mechanism in the pathogenesis of drug-induced autoimmunity is based on DNA hypomethylation, an epigenetic process where changes in gene expression occur without an alteration in the DNA sequence. Several studies have shown that both procainamide and hydralazine inhibit DNA methylation in T cells, thereby changing the T cell gene expression profiles and T cell function. Inhibition of DNA methylation is associated with an overexpression of lymphocyte functional antigen 1 (LFA-1 or CD11a), an adhesion molecule with important functions in co-stimulation and stabilisation of the interaction between $T$ cells and antigen-presenting cells, resulting in hyper-responsiveness of $\mathrm{T}$ cells to signals that normally do not trigger activation, e.g., self-antigens with low-affinity binding to T-cell receptors (TCRs) ${ }^{72-75}$. Finally, drug-induced autoimmunity may be due to interference with central immune tolerance. The thymus provides tolerance by deleting auto-reactive $T$ cells during thymus maturation. Two studies in mouse models have shown that during positive selection of thymocytes, intra-thymic injections of reactive drug metabolites interfere with tolerance to low-affinity (self) endogenous antigens that are normally presented by $\mathrm{MHC}$ on thymic epithelial cells. As a result, autoreactive T cells are migrated from the thymus to the periphery where they provide Th cell function to B cells with the potential to produce autoantibodies 76,77 .

As described earlier, the confirmation of drug-induced autoimmunity may be achieved by re-exposure to the drug. However, deliberate rechallenge is usually not advocated, as patients are likely to have a severe response when they are re-exposed to the drug. As straightforward as this approach seems, making a diagnosis of drug-induced autoimmunity often proves to be challenging in clinical practice ${ }^{32}$. Many patients, especially elderly patients, may have multiple diagnoses and treatments, or have a medical history that is not well documented, which could make it difficult to establish an association between the drug in question and the patient's symptoms. Furthermore, various drug-induced autoimmune mechanisms may involve processes that are not readily or rapidly reversed, suggesting that withdrawal of the suspected offending drug may not modify the clinical course of the disease ${ }^{44}$ as has been found in lupus-like syndrome where antinuclear antibodies (ANA) and elastase ANCA are 
persistently positive many months after drug discontinuation 59,78. Another difficulty that may be encountered in setting a diagnosis of drug-induced autoimmunity is the nature of autoimmune disease. Although each autoimmune disease is unique, many share features with other diseases, making it difficult to establish whether the presence of autoantibodies is part of the underlying disease or attributable to the drug. For example, anti-histone antibodies are not specific for drug-induced autoimmune diseases but may also be detected in "idiopathic" rheumatoid arthritis (RA) 79 . There are a number of drugs (e.g. statins) that have been prescribed as powerful modulators of disease processes (e.g. cardiovascular disease), and as a consequence physicians and patients are understandably concerned when the use of these drugs are discontinued without proof of an adverse effect 44 .

Drug-induced autoimmune disorders are of major concern and often reason for withdrawal from the market, especially when high numbers of serious adverse drug reactions (ADRs) are identified during post-marketing surveillance. In daily practice, it is rather difficult to detect these side effects because they are relatively uncommon, its symptoms are generally mild to moderate, appear after prolonged exposure to the drug, and may occasionally persist, despite drug discontinuation ${ }^{44}$. According to a French survey using the French pharmacovigilance system, approximately $0.2 \%$ of the ADRs are reported or published in literature as systemic autoimmune ADRs 80 . Not only for pharmacovigilance working parties but also for physicians it is a major challenge to set a diagnosis of drug-induced autoimmunity. Nevertheless, it must be emphasised that drug-induced autoimmunity can be chronic, with long-term morbidity, and therefore may potentially impose a heavy burden on public health.

\section{SYSTEMIC AUTOIMMUNITY}

Since the initial description of drug-induced autoimmunity, more than half a century ago, a wide range of pharmacologic agents have been implicated in interfering with specific functions of the immune system and induce autoimmune disorders. Drug-induced autoimmune disorders often exhibit heterogeneity in clinical features and drugs are capable of eliciting an immune response to certain auto-antigens. These symptoms of drug-induced autoimmunity show some resemblance to typical systemic autoimmune diseases, e.g., vasculitis 46, scleroderma 81 , sjögren's syndrome 82 , dermato- and polymyositis 83,84 , and lupus-like syndrome 40,45 . Indeed, lupus-like syndrome has been associated with nearly 100 drugs, including the aforementioned drugs hydralazine, procainamide, chlorpromazine, isoniazid, methyldopa, minocycline and quinidine 85 . On the other hand, several organ-specific autoimmune diseases, e.g., membraneous glomerulonephritis 86, autoimmune hepatitis 87 , thyroiditis 88 , haemolytic anamia 89 , myasthenia gravis 90 , and the skin 
diseases pemphigus and pemphigoid ${ }^{91-93}$ have been reported to be associated with drug intake. In this thesis, we focus on the so-called systemic autoimmune diseases.

\section{CARDIOVASCULAR DRUGS}

As previously described, CVD is the leading cause of death in the Netherlands and in the world 1,2. Currently, important interventions to prevent and to treat CVD are available, e.g., pharmacological treatment of elevated low density lipoprotein (LDL) cholesterol levels 94 , elevated blood pressure 95-97 and inhibiting platelet function 98,99 with statins, anti-hypertensive agents (thiazides, beta blockers, calcium channel blockers, angiotensin converting enzyme (ACE) inhibitors, or angiotensin II receptor blockers (ARBs)) and aspirin, respectively. Statins, ACE inhibitors and ARBs were listed in the top 20 of most prescribed drugs in the USA in the year 2011. With more than 260 million prescriptions for statins and other lipid-lowering drugs, these drugs were, after antidepressant drugs, the most prescribed drugs whereas ACE inhibitors and ARBs were listed as the fifth and sixteenth most prescribed drug with 164 and 86 million dispensed prescriptions respectively 100. Since statins, ACE inhibitors and ARBs are widely prescribed for patients with cardiovascular diseases, and have shown to exert immunomodulatory effects ${ }^{4,14}$, we focus on these types of cardiovascular drugs in this thesis.

\section{STATINS}

Statins, 3-hydroxy-3-methylglutaryl coenzyme A (HMG-CoA) reductase inhibitors have been extensively used in the last decades to reduce cardiovascular morbidity and mortality. Several clinical trials have demonstrated that this reduction is due to lowering serum lipid levels, specifically low-density lipoprotein cholesterol (LDL-C), non-high-density lipoprotein cholesterol (non-HDL-C) and apolipoprotein B which are risk factors in cardiovascular diseases 101,102. Statins were first identified as a successful therapy in 1976 by Endo et al. 103,104. A subsequent study by Brown and Goldstein, winners of the 1985 Nobel Prize in Medicine for discovering the LDL receptor and its role in regulating cholesterol 105, demonstrated that statins act as inhibitors of HMG-CoA reductase, an enzyme that catalyses the rate-limiting step of the cholesterol synthesis pathway in the liver, resulting in an upregulation of LDL receptors in the liver and thereby lowering blood cholesterol levels 106,107. Inhibition of this enzyme in the mevalonate or cholesterol synthesis pathway reduces the downstream biosynthesis of cholesterol and other intermediate metabolites (figure 1) 108, 


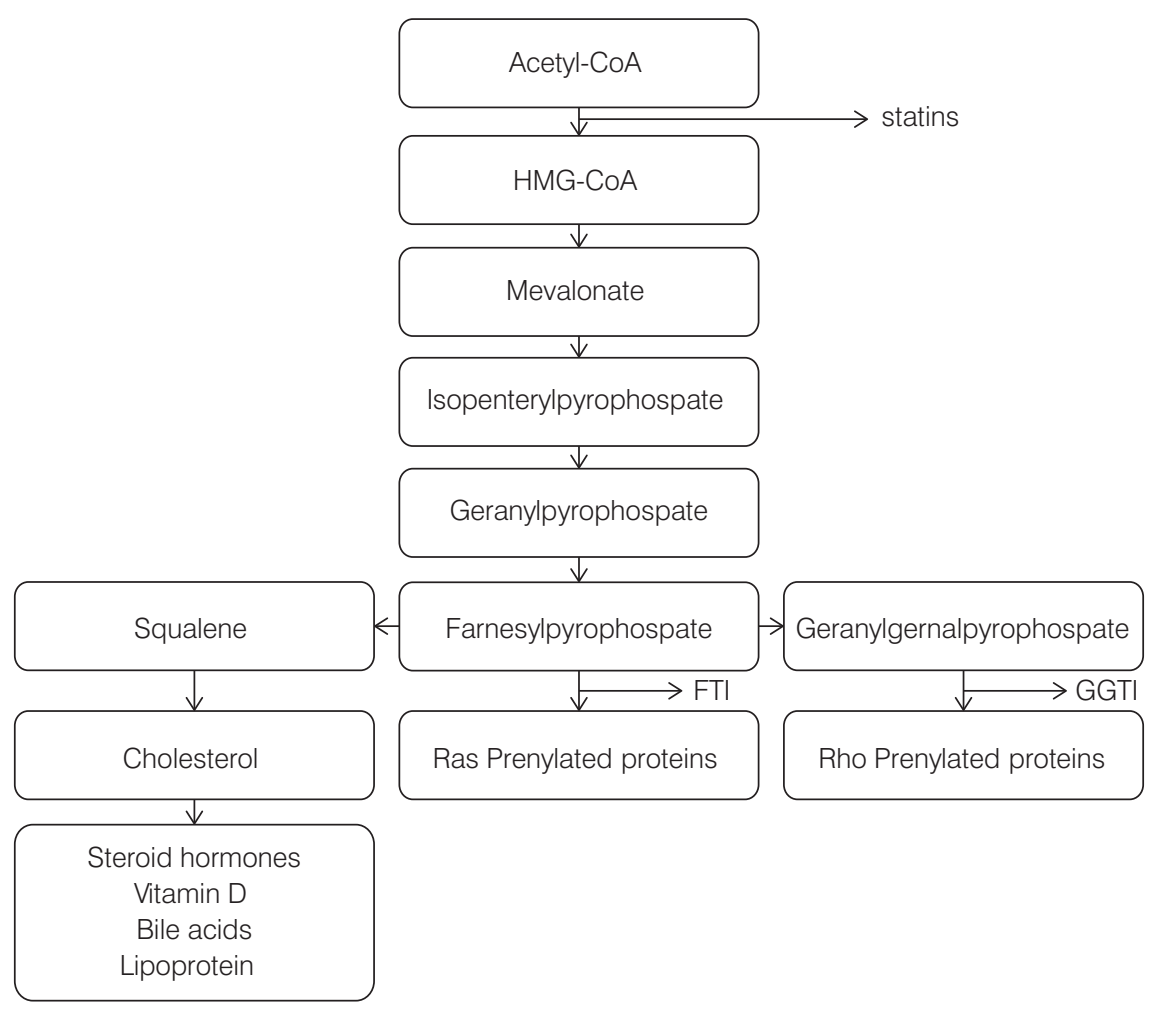

Figure 1 The cholesterol biosynthetic pathway.

HMG-CoA, 3-hydroxy-3-methylglutaryl coenzyme A; FTI, farnesyltransferase inhibitor; GGTI, geranylgeranyl transferase inhibitor.

including the isoprenoids farnesyl and geranylgeranyl pyrophosphate, which are important for prenylation (lipid post-translational modification) of cellular proteins, such as the GTP-binding proteins Ras and Rho ${ }^{109}$. Members of the Ras and Rho family are involved in regulation of cell growth, cell-to-cell signalling, cell proliferation and apoptosis, and have been proposed to have antineoplastic potential 110-112. As described above, statins inhibit the Ras and Rho isoprenylation, thereby resulting in an accumulation of the inactive forms of these proteins in the cytoplasm ${ }^{113}$. This inhibitory effect of statins in cellular metabolism modulates the signalling pathways that involve endothelial nitric oxide synthase (eNOS) 114,115 , tissue plasminogen activator and plasminogen activator inhibitor 1 116,117, endothelin 1 118,119, and apoptosis of the smooth muscle cells 120,121. Several studies have shown that statins reduce platelet activation and adhesion 122,123, and decrease the biosynthesis of thromboxane A2 
124,125. In addition, statins effectively inhibit secretion of pro-inflammatory cytokines, tumor necrosis factor alpha (TNF-a), IL- $\beta$, IL-6 and IL-8 126-128, chemokine monocyte chemoattractant protein 1 (MCP1) 129,130 and matrix metalloproteinase 131,132. Several clinical trials addressed the anti-inflammatory effects of statins by measuring C-reactive protein (CRP), a biomarker of innate immune responses that has been rigorously established as a risk indicator for CVD 133-137. Indeed, results from these clinical trials showed that statins decrease levels of CRP 134-136. Since statin therapy reduces the incidence of acute and chronic rejection in heart and renal transplant patients 138-141, the immunomodulating effects have been further studied. Statins have been reported to suppress interferon- $\gamma$ (IFN- $\gamma$ )-inducible expression of major histocompatibility complex (MHC) class II proteins in endothelial cells, as well as in monocytes/macrophages and $\mathrm{T}$ cells, but do not affect dendritic cells and B-lymphocytes 14,142,143. Another beneficial effect of statins is the effect on the Th1/ Th2 balance, as demonstrated in a murine model of Experimental Allergic Encephalomyelitis (EAE), an animal model mimicking $\mathrm{MS}^{16}$. In this model, statin treatment induced secretion of Th2 cytokines (IL-4, IL-5 and IL-10) and transforming factor $\beta$ (TGF- $\beta$ ), whereas secretion of Th1 cytokines (IL-2, IL-12, IFN- $\gamma$ and TNF- $\alpha$ ) were suppressed. Similarly, in a murine model of autoimmune retinal disease and in patients with acute myocardial infarction, statins decreased the production of IFN- $\gamma$, whereas no effect on Th2 cytokine production was observed 144,145 . Previous studies indicated that statins may enhance regulatory $T$ cell (Treg) responses by promoting chemokine-dependent recruitment into inflammatory sites or by inducing the transcription factor FoxP3 146,147. Recently, it has been suggested that statins skew T cell differentiation towards Tregs and away from pro-inflammatory Th17 cells via geranylgeranylation of proteins, resulting in promoting Treg differentiation in the periphery, while blocking Th17 cell differentiation 148. Statins also down-regulate expression of the co-stimulatory molecule CD40 in various cell types, e.g., endothelial cells, smooth muscle cells and macrophages 149,150. Interestingly, certain types of statins selectively block the $\beta-2$ integrin, leukocyte function antigen-1 (LFA-1), thereby blocking binding to intercellular adhesion molecule-1 (ICAM-1) and thus reducing T cell activation ${ }^{17}$. Taken together, these data support that statins possess anti-inflammatory and immunomodulating properties that may be beneficial in the treatment of immune-mediated disorders other than atherosclerosis. Indeed, beneficial effects of statins were observed in clinical trials and experimental models of RA, MS, SLE, systemic sclerosis and antiphospholipid syndrome 18-23,151-153.

In general, statins are considered to be safe although the market withdrawal of cerivastatin has demonstrated that some serious adverse effects were not detected during clinical trials. This is mostly true because rare adverse effects of statins appear only many months after starting the therapy 83,154 . The most commonly reported side effects of statins are marked elevation of liver enzymes and muscular abnormalities 
155. The spectrum of statin-associated muscular side effects ranges from the more common but less severe myalgia (5-10\%) to the less common but more severe myopathy $(0.1 \%)$ and its potentially fatal complication, rhabdomyolysis (0.01\%) 156 . Moreover, an increasing number of case reports suggest that prolonged use of statins may also trigger auto-immune diseases such as SLE, dermatomyositis, polymyositis, vasculitis, polymyalgia rheumatica, myasthenia gravis, guillain barre, autoimmune hepatitis and autoimmune skin diseases 83,92,157-164. Three pathogenic mechanisms may be suspected in statin-associated autoimmunity. First, statins are potent pro-apoptotic agents and may trigger or exacerbate cellular apoptosis ${ }^{165}$, thereby releasing nuclear antigens into the circulation, which may foster the production of pathogenic autoantibodies 78 . Second, as described above, it has been suggested that statins induce a shift from a Th1 to Th2 immune response by their direct effect on T cells. Promoting a shift from Th1 to Th2 immune responses may dysregulate the immune homeostasis and can lead to the breakdown of self-tolerance, precipitating autoimmunity $16,83,166$. Third, statins may enhance the formation of extracellular traps (ETs) by phagocytic cells such as neutrophils, mast cells and eosinophils ${ }^{167}$. These ETs by phagocytic cells have been shown not only to exert antibacterial effects but may also provoke inflammation and pathological release of ETs in inflammatory and autoimmune diseases such as psoriasis, vasculitis, SLE and lupus nephritis ${ }^{168-173 .}$

\section{ANGIOTENSIN-CONVERTING ENZYME INHIBITORS AND ANGIOTENSIN II RECEPTOR BLOCKERS}

Anti-hypertensive drugs, ACE inhibitors and ARBs, are widely used in the treatment of hypertension ${ }^{174}$, cardiac failure ${ }^{175}$, left ventricular systolic dysfunction ${ }^{176}$, acute myocaridial infarction 177 and as antiproteinuric drugs in non- and diabetic nephropathy 178,179 . The history of ACE inhibitors and ARBs can be traced back to the fifties. In 1954, Skeggs et al. recognised for the first time substrates participating in the renin-angiotensin system, and in 1956, they reported the purification of an enzyme which converted inactive angiotensin I to the active vasoconstrictor angiotensin II in the presence of chloride ions from horse plasma ${ }^{180-182}$. They named this enzyme ACE. It appeared that ACE not only cleaves angiotensin I but is also responsible for the degradation of bradykinin 183,184. In 1965, Ferreira showed that a non-toxic peptide of the venom from the Brazilian viper, Bothrops jararaca, enhanced the effects of bradykinin: smooth muscle contraction, hypotension and increased capillary permeability ${ }^{185}$. Based on this finding, a novel class of anti-hypertensive agents was developed by Ondetti and Cushman in the early seventies: the angiotensinconverting enzyme (ACE) inhibitors 186,187. Captopril was the first oral ACE inhibitor introduced into clinical practice and was approved by the USA Food and Drug 
Administration (FDA) in 1981 188. In the subsequent years, orally active ARBs have been developed as an alternative to inhibit the effects of angiotensin II. Losartan was the first ARB discovered in 1986 and approved by the FDA in 1995189.

ACE inhibitors inhibit ACE which converts angiontensin I to vasoconstrictor angiotensin II, thereby producing vasodilation ${ }^{190}$. In contrast to ACE inhibitors, ARBs block the effects of angiotensin II through binding to angiotensin II type 1 receptors 191. The efficacy of both ACE inhibitors and ARBs in hypertension, diabetic nephropathy and congestive heart failure is well established, and generally, ACE inhibitors and ARBs are well tolerated, with a low incidence of side effects ${ }^{192}$. ACE inhibitors and ARBs may induce skin rashes, angio-oedema, diarrhoea, cough, upper respiratory tract infection, dizziness and renal dysfunction 193-196, although ARBs produce these side effects to a much lesser extent than ACE inhibitors. As cough is one of the most frequent side effects associated with ACE inhibitors, several studies with ARBs have specifically addressed this side effect. The frequency of cough in ARB users was significantly lower than that observed in ACE inhibitor users 197,198 , but was comparable to patients that are treated with a diuretic or placebo 197-202. The adverse effect profile of ARBs is comparable to what is seen in the placebo groups 203-205, no class-specific and dose-dependent adverse effects can be attributed to the drug itself 205. However, concerns have been raised about possible associations between ARBs and increased risks of cancer 206. The mechanism by which ARBs increase the occurrence of cancer is unknown. In experimental studies, using cancer cell lines, it has been implicated that the renin-angiotensin system is involved in the regulation of cell proliferation, tumour growth, angiogenesis and metastasis 207,208. In one in vivo study of Walther et al., it was shown that by blocking the angiotensin I receptor with losartan, a stimulation of tumour angiogenesis was induced 209.

Recently, much interest has focused on the potential for ACE inhibitors and ARBs to exert anti-inflammatory and immunomodulating properties ${ }^{4-12}$. The immunomodulatory properties of ACE inhibitors and ARBs have been attributed to several mechanisms, including anti-proliferative activity 210,211, inhibition of metalloproteases 210 and elevation of immunomodulatory prostaglandins 212. In two studies of RA patients treated with ACE inhibitors, decreased levels of CRP were found whereas another study found decreased levels of erythrocyte sedimentation rate (ESR) in RA patients treated with ARBs 10-12. Since the ACE enzyme has been shown to be involved in immune functions 213,214 and is elevated in inflammatory conditions 215,216, inhibition of ACE itself may have immune suppressive effects. Furthermore, ACE inhibitors and ARBs may alter cellular immunity, and inhibit adhesion molecule regulation, chemotaxis and production of several proinflammatory cytokines, e.g., TNF-a, IL-1, IL-6 and IL-12 4-9. In a study of angiotensin-II-infused hypertensive rats with kidney injury, the imbalance between Th1 and Th2 cells was corrected by treatment with 
ARBs 217. In addition, in the EAE mouse model it was demonstrated that ACE inhibitors and ARBs suppress the auto-reactive Th1 and Th17 cells and promote regulatory T cells ${ }^{4}$. These beneficial effects of ACE inhibitors and/or ARBs result in the prevention of nephropathy of insulin-dependent diabetes mellitus 178,218 and anti-inflammatory effects in RA 10-12. Similarly, ACE inhibitors and ARBs successfully suppress inflammation in experimental conditions, including experimental lupus disease 219,220, autoimmune myocarditis 221 and encephalomyelitis (EAE) 222.

On the other hand, it has been demonstrated that the immunomodulating effects of ACE inhibitors or ARBs may facilitate autoimmune responses. Several case reports have suggested that treatment with ACE inhibitors and/or ARBs may induce the development of autoantibodies 223-225 and autoimmune diseases, e.g., lupus-like disease, vasculitis and pemphigus 226-247. It has been demonstrated that ACE inhibitors enhance Trypanosoma cruzi infection of human monocytes, reduce the expression of the modulatory cytokine IL-10 while inducing Th17 cells ${ }^{248}$, which have been shown to be associated with autoimmunity 249 . Finally, two studies have shown that ACE inhibitors block activation-induced apoptosis in T cells 250,251, supporting the idea that ACE inhibitors may hamper clonal deletion and interfere with maintenance of self-tolerance, thereby precipitating autoimmunity.

\section{OBJECTIVE OF THE THESIS}

Several studies demonstrated that statins, ACE inhibitors and ARBs are effective in reducing the risk of cardiovascular morbidity and mortality 101,102,176,177. In addition to their effects on cholesterol levels and blood pressure, recent studies have shown that these agents have anti-inflammatory and immunomodulatory properties, which also may contribute to the beneficial effects of these drugs in the treatment of cardiovascular disease and certain autoimmune diseases $4-23$. While much is known about the beneficial effects of statins, ACE inhibitors and ARBs, the use of these drugs may also adversely influence immune regulation and may therefore facilitate the development of autoimmune diseases. In daily practice, it is rather difficult to detect these side effects as they are relatively uncommon, may be less severe, appear after prolonged use, and may induce persistent immune deviations after cessation of these drugs $78,252,253$. Therefore, the objective of this thesis is to assess the association between prolonged use of cardiovascular drugs, particularly statins, ACE inhibitors, and ARBs, and the incidence of autoimmune disorders. 


\section{OUTLINE OF THIS THESIS}

This thesis contains eight studies described in four chapters. The present chapter, chapter 1, introduces the topic of this thesis.

In order to strengthen our hypothesis that certain cardiovascular drugs facilitate autoimmune disorders, we have performed three studies, using data on spontaneously reported adverse drug reactions collected during use of statins in daily clinical practice (chapter 2). In chapter 2.1 and chapter 2.2, respectively, we have assessed the association between statin use and the occurrence of autoimmune disorders by carrying out a case/non-case approach based on individual case safety reports of adverse drug reactions. Chapter 2.1 focuses on lupus-like syndrome while chapter 2.2 focuses on polymyalgia rheumatica (PMR). One of the individual case safety reports included in the study presented in chapter 2.2, we were able to collect additional medical information from the reporter. In chapter 2.3, we have described the case reports' nature of statin-associated PMR and giant cell arteritis (GCA) in more detail to illustrate the possible causality of statin-associated PMR/GCA.

As the spontaneous reporting database is primarily used for signal detection (hypothesis strengthening) purposes and not for hypothesis testing, we have tested our hypothesis in electronic health record databases. In chapter 3, we focus on cardiovascular drug use and the incidence of RA, using population-based studies. In chapter 3.1, the risk of developing RA associated with different exposure aspects (duration, dose, type and potency) of statin use has been evaluated by a case-control study. Chapter 3.2 describes the association between the use of statins and the risk of RA, with a special focus on describing the patterns of the hazard rates (i.e. the absolute risks) of RA with changes in statin exposure by conducting a retrospective cohort study. Another approach of testing our hypothesis was to perform a study where we have examined whether the use of ACE inhibitors or ARBs is associated with incident RA. In chapter 3.3, the use of ACE inhibitors or ARBs and incident RA have been investigated in a case-control study among patients with anti-hypertensive drugs.

Chapter 4 focuses on statin use and (auto) immunity. We have assessed the effects of statins on innate and adaptive immunity, and self-tolerance by measuring serological levels of CRP, neopterin and IgE antibodies, and the presence of autoantibodies in serum collected from the general population cross-sectionally and longitudinally.

Chapter 5 addresses the causal relationship of the findings presented in chapter 3.1 and 3.2 by evaluating the effects of statin administration on arthritis in the collagen type II-induced arthritis (CIA) mouse model.

Finally, in chapter 6 the findings presented in this thesis are discussed and recommendations are given for clinical practice and future research. 


\section{REFERENCES}

1. Vaartjes I, Van Dis I, Visseren F, Bots M. [Hart- en vaatziekten in Nederland 2010. Cijfers over leefstijl- en risicofactoren, ziekte en sterfte.] The Hague, The Netherlands: Nederlandse Hartstichting, 2010.

2. World Health Organisation, World Heart Federation, World Stroke Organisation. Global atlas on cardiovascular disease prevention and control. Policies, strategies and interventions. Geneva, Switzerland: World Health Organisation, 2011.

3. Wald NJ, Law MR. A strategy to reduce cardiovascular disease by more than $80 \%$. BMJ 2003; 326(7404): 1419.

4. Platten M, Youssef S, Hur EM, et al. Blocking angiotensin-converting enzyme induces potent regulatory T cells and modulates TH1- and TH17-mediated autoimmunity. Proc Natl Acad Sci U S A 2009; 106(35): 14948-53.

5. Sukumaran V, Watanabe K, Veeraveedu PT, et al. Olmesartan, an AT1 antagonist, attenuates oxidative stress, endoplasmic reticulum stress and cardiac inflammatory mediators in rats with heart failure induced by experimental autoimmune myocarditis. Int J Biol Sci 2011; 7(2): 154-67.

6. Ruiz-Ortega M, Bustos C, Hernandez-Presa MA, Lorenzo O, Plaza JJ, Egido J. Angiotensin II participates in mononuclear cell recruitment in experimental immune complex nephritis through nuclear factor-kappa B activation and monocyte chemoattractant protein-1 synthesis. J Immunol 1998; 161(1): 430-9.

7. Constantinescu CS, Goodman DB, Ventura ES. Captopril and lisinopril suppress production of interleukin-12 by human peripheral blood mononuclear cells. Immunol Lett 1998; 62(1): 25-31.

8. Fukuzawa M, Satoh J, Sagara M, et al. Angiotensin converting enzyme inhibitors suppress production of tumor necrosis factor-alpha in vitro and in vivo. Immunopharmacology 1997; 36(1): 49-55.

9. Schindler R, Dinarello CA, Koch KM. Angiotensin-converting-enzyme inhibitors suppress synthesis of tumour necrosis factor and interleukin 1 by human peripheral blood mononuclear cells. Cytokine 1995; 7(6): 526-33

10. Martin MF, Surrall KE, McKenna F, Dixon JS, Bird HA, Wright V. Captopril: a new treatment for rheumatoid arthritis? Lancet 1984; 1(8390): 1325-8

11. Bird HA, Le Gallez P, Dixon JS, et al. A clinical and biochemical assessment of a nonthiol ACE inhibitor (pentopril; CGS-13945) in active rheumatoid arthritis. J Rheumatol 1990; 17(5): 603-8.

12. Perry ME, Chee MM, Ferrell WR, Lockhart JC, Sturrock RD. Angiotensin receptor blockers reduce erythrocyte sedimentation rate levels in patients with rheumatoid arthritis. Ann Rheum Dis 2008; 67(11): 1646-7.

13. Ridker PM, Danielson E, Fonseca FA, et al. Reduction in C-reactive protein and LDL cholesterol and cardiovascular event rates after initiation of rosuvastatin: a prospective study of the JUPITER trial. Lancet 2009; 373(9670): 1175-82.

14. Arnaud C, Braunersreuther V, Mach F. Toward immunomodulatory and anti-inflammatory properties of statins. Trends Cardiovasc Med 2005; 15(6): 202-6.

15. Kwak BR, Mulhaupt F, Mach F. Atherosclerosis: anti-inflammatory and immunomodulatory activities of statins. Autoimmun Rev 2003; 2(6): 332-8.

16. Youssef S, Stuve O, Patarroyo JC, et al. The HMG-CoA reductase inhibitor, atorvastatin, promotes a Th2 bias and reverses paralysis in central nervous system autoimmune disease. Nature 2002; 420(6911): 78-84.

17. Weitz Schmidt G, Welzenbach K, Brinkmann V, et al. Statins selectively inhibit leukocyte function antigen-1 by binding to a novel regulatory integrin site. Nat Med 2001; 7(6): 687-92.

18. Leung BP, Sattar N, Crilly A, et al. A novel anti-inflammatory role for simvastatin in inflammatory arthritis. J Immunol 2003; 170(3): 1524-30.

19. McCarey DW, Mclnnes IB, Madhok R, et al. Trial of Atorvastatin in Rheumatoid Arthritis (TARA): double-blind, randomised placebo-controlled trial. Lancet 2004; 363(9426): 2015-21.

20. Neuhaus O, Stuve O, Zamvil SS, Hartung HP. Are statins a treatment option for multiple sclerosis? Lancet Neurol 2004; 3(6): 369-71

21. Vollmer T, Key L, Durkalski V, et al. Oral simvastatin treatment in relapsing-remitting multiple sclerosis. Lancet 2004; 363(9421): 1607-8. 
22. Ferreira GA, Navarro TP, Telles RW, Andrade LEC, Sato El. Atorvastatin therapy improves endothelialdependent vasodilation in patients with systemic lupus erythematosus: an 8 weeks controlled trial. Rheumatology 2007; 46(10): 1560-5.

23. López-Pedrera C, Aguirre MA, Barbarroja N, Cuadrado MJ. Accelerated atherosclerosis in systemic lupus erythematosus: role of proinflammatory cytokines and therapeutic approaches. J Biomed Biotechnol 2010; 2010: pii:607084.

24. Lleo A, Invernizzi P, Gao B, Podda M, Gershwin ME. Definition of human autoimmunity--autoantibodies versus autoimmune disease. Autoimmun Rev 2010; 9(5): A259-66.

25. Kuchroo V, Ohashi P, Sartor RB, Vinuesa C. Dysregulation of immune homeostasis in autoimmune diseases. Nat Med 2012; 18(1): 42-7.

26. Molina $V$, Shoenfeld $Y$. Infection, vaccines and other environmental triggers of autoimmunity. Autoimmunity 2005; 38(3): 235-45.

27. Singh N, Singh U, Singh B, Price R, Nagarkatti M, Nagarkatti P. Activation of aryl hydrocarbon receptor (AhR) leads to reciprocal epigenetic regulation of FoxP3 and IL-17 expression and amelioration of experimental colitis. PLoS ONE 2011; 6(8): e23522.

28. Todd J. D'oh! genes and environment cause Crohn's disease. Cell 2010; 141(7): 1114-6.

29. Marrack P, Kappler J, Kotzin BL. Autoimmune disease: why and where it occurs. Nat Med 2001; 7(8): 899-905.

30. Klareskog L, Malmström V, Lundberg K, Padyukov L, Alfredsson L. Smoking, citrullination and genetic variability in the immunopathogenesis of rheumatoid arthritis. Semin Immunol 2011; 23(2): 92-8.

31. WHO Collaborating Centre for Immunotoxicology and Allergic Hypersensitivity. Principles and methods for assessing autoimmunity associated with exposure to chemicals: Environmental Health Criteria. Bilthoven, The Netherlands: World Health Organisation, 2006.

32. Dedeoglu F. Drug-induced autoimmunity. Curr Opin Rheumatol 2009; 21(5): 547-51.

33. Ditto A. Drug allergy. In: Grammer L, Greenberger P, editors. Patterson's Allergic diseases. 6th ed. Philadelphia: Lippincott Williams \& Wilkins; 2002. p.295-334.

34. Jick H. Adverse drug reactions: the magnitude of the problem. J Allergy Clin Immunol 1984; 74(4): 555-7.

35. Lazarou J, Pomeranz BH, Corey PN. Incidence of adverse drug reactions in hospitalized patients: a meta-analysis of prospective studies. JAMA 1998; 279(15): 1200-5.

36. Einarson TR. Drug-related hospital admissions. Ann Pharmacother 1993; 27(7-8): 832-40.

37. Hoffmann B. Sensitivity to sulfadiazine resembling acute disseminated lupus erythematosus. Arch Dermatol Syph 1945; 51: 190-2.

38. Alarcón Segovia D, Wakim KG, Worthington JW, Ward LE. Clinical and experimental studies on the hydralazine syndrome and its relationship to systemic lupus erythematosus. Medicine 1967; 46(1): 1-33.

39. Ullman S, Wiik A, Kobayasi T, Halberg P. Drug-induced lupus erythematosus syndrome. Immuno-pathological, electronmicroscopical and serological studies. Acta Derm Venereol 1974; 54(5): 387-90.

40. Borchers AT, Keen CL, Gershwin ME. Drug-induced lupus. Ann N Y Acad Sci 2007; 1108: 166-82.

41. Mongey A, Hess E. Drug insight: autoimmune effects of medications-what's new? Nat Clin Pract Rheumatol 2008; 4(3): 136-44. doi:10.1038/ncprheum0708.

42. Mor A, Pillinger M, Wortmann R, Mitnick H. Drug-induced arthritic and connective tissue disorders. Semin Arthritis Rheum 2008; 38(3): 249-64.

43. Vedove CD, Del Giglio M, Schena D, Girolomoni G. Drug-induced lupus erythematosus. Arch Dermatol Res 2009; 301(1): 99-105.

44. Olsen N. Drug-induced autoimmunity. Best Pract Res Clin Rheumatol 2004; 18(5): 677-88.

45. Vasoo S. Drug-induced lupus: an update. Lupus 2006; 15(11): 757-61.

46. Wiik A. Drug-induced vasculitis. Curr Opin Rheumatol 2008; 20(1): 35-9.

47. Hess E. Drug-related lupus. N Engl J Med 1988; 318(22): 1460-2.

48. Dolman KM, Gans RO, Vervaat TJ, et al. Vasculitis and antineutrophil cytoplasmic autoantibodies associated with propylthiouracil therapy. Lancet 1993; 342(8872): 651-2.

49. Choi HK, Merkel PA, Walker AM, Niles JL. Drug-associated antineutrophil cytoplasmic antibody-positive vasculitis: prevalence among patients with high titers of antimyeloperoxidase antibodies. Arthritis Rheum 2000; 43(2): 405-13 
50. Rubin RL. Drug-induced lupus. Toxicology 2005; 209(2): 135-47.

51. Uetrecht J. Current trends in drug-induced autoimmunity. Autoimmun Rev 2005; 4(5): 309-14.

52. Marzano AV, Vezzoli P, Crosti C. Drug-induced lupus: an update on its dermatologic aspects. Lupus 2009; 18(11): 935-40

53. Griem P, Wulferink M, Sachs B, González JB, Gleichmann E. Allergic and autoimmune reactions to xenobiotics: how do they arise? Immunol Today 1998; 19(3): 133-41.

54. Jiang X, Khursigara G, Rubin RL. Transformation of lupus-inducing drugs to cytotoxic products by activated neutrophils. Science 1994; 266(5186): 810-3.

55. Popa ER, Franssen CF, Limburg PC, Huitema MG, Kallenberg CG, Tervaert JW. In vitro cytokine production and proliferation of $\mathrm{T}$ cells from patients with anti-proteinase 3- and antimyeloperoxidase-associated vasculitis, in response to proteinase 3 and myeloperoxidase. Arthritis Rheum 2002; 46(7): 1894-904.

56. Abdulahad WH, Lamprecht P, Kallenberg CG. T-helper cells as new players in ANCA-associated vasculitides. Arthritis Res Ther 2011; 13(4): 236.

57. Shapiro LE, Uetrecht J, Shear NH. Minocycline, perinuclear antineutrophilic cytoplasmic antibody, and pigment: the biochemical basis. J Am Acad Dermatol 2001; 45(5): 787-9.

58. Pillinger M, Staud R. Wegener's granulomatosis in a patient receiving propylthiouracil for Graves' disease. Semin Arthritis Rheum 1998; 28(2): 124-9.

59. Nässberger L, Johansson AC, Björck S, Sjöholm AG. Antibodies to neutrophil granulocyte myeloperoxidase and elastase: autoimmune responses in glomerulonephritis due to hydralazine treatment. $J$ Intern Med 1991; 229(3): 261-5

60. Slot M, Links T, Stegeman C, Tervaert JWC. Occurrence of antineutrophil cytoplasmic antibodies and associated vasculitis in patients with hyperthyroidism treated with antithyroid drugs: A long-term followup study. Arthritis Rheum 2005; 53(1): 108-13

61. Ablin J, Verbovetski I, Trahtemberg U, Metzger S, Mevorach D. Quinidine and procainamide inhibit murine macrophage uptake of apoptotic and necrotic cells: a novel contributing mechanism of drug-induced-lupus. Apoptosis 2005; 10(5): 1009-18.

62. Navratil JS, Ahearn JM. Apoptosis, clearance mechanisms, and the development of systemic lupus erythematosus. Curr Rheumatol Rep 2001; 3(3): 191-8.

63. Hieronymus T, Grötsch P, Blank N, et al. Chlorpromazine induces apoptosis in activated human lymphoblasts: a mechanism supporting the induction of drug-induced lupus erythematosus? Arthritis Rheum 2000; 43(9): 1994-2004.

64. Remijsen Q, Kuijpers TW, Wirawan E, Lippens S, Vandenabeele P, Vanden Berghe T. Dying for a cause: NETosis, mechanisms behind an antimicrobial cell death modality. Cell Death Differ 2011; 18(4): 581-8.

65. Brinkmann V, Reichard U, Goosmann C, et al. Neutrophil extracellular traps kill bacteria. Science 2004; 303(5663): 1532-5.

66. Ravichandran K. Find-me and eat-me signals in apoptotic cell clearance: progress and conundrums. J Exp Med 2010; 207(9): 1807-17.

67. Fuchs $T$, Abed $U$, Goosmann C, et al. Novel cell death program leads to neutrophil extracellular traps. J Cell Biol 2007; 176(2): 231-41.

68. Hakkim A, Fürnrohr B, Amann K, et al. Impairment of neutrophil extracellular trap degradation is associated with lupus nephritis. Proc Natl Acad Sci U S A 2010; 107(21): 9813-8

69. Kessenbrock K, Krumbholz M, Schönermarck U, et al. Netting neutrophils in autoimmune small-vessel vasculitis. Nat Med 2009; 15(6): 623-5.

70. Lande R, Ganguly D, Facchinetti V, et al. Neutrophils activate plasmacytoid dendritic cells by releasing self-DNA-peptide complexes in systemic lupus erythematosus. Science translational medicine 2011; 3(73): 73ra19.

71. Nakazawa D, Tomaru U, Suzuki A, et al. Abnormal conformation and impaired degradation of propylthiouracil-induced neutrophil extracellular traps: Implications of disordered neutrophil extracellular traps in a rat model of myeloperoxidase antineutrophil cytoplasmic antibody-associated vasculitis. Arthritis Rheum 2012; 64(11): 3779-87.

72. Deng C, Lu Q, Zhang Z, Rao T, Attwood J, Yung R, Richardson B. Hydralazine may induce autoimmunity by inhibiting extracellular signal-regulated kinase pathway signaling. Arthritis Rheum 2003; 48(3): 746-56. 
73. Balada E, Ordi Ros J, Vilardell Tarrés M. DNA methylation and systemic lupus erythematosus. Ann N Y Acad Sci 2007; 1108: 127-36.

74. Yung R, Powers D, Johnson K, et al. Mechanisms of drug-induced lupus. II. T cells overexpressing lymphocyte function-associated antigen 1 become autoreactive and cause a lupuslike disease in syngeneic mice. J Clin Invest 1996; 97(12): 2866-71.

75. Zhou Y, Lu Q. DNA methylation in T cells from idiopathic lupus and drug-induced lupus patients. Autoimmunity Rev 2008; 7(5): 376-83.

76. Kretz Rommel A, Duncan SR, Rubin RL. Autoimmunity caused by disruption of central T cell tolerance. A murine model of drug-induced lupus. J Clin Invest 1997; 99(8): 1888-96.

77. Kretz Rommel A, Rubin RL. Disruption of positive selection of thymocytes causes autoimmunity. Nat Med 2000; 6(3): 298-305.

78. Noël B. Statins and lupus erythematosus. Rheumatology 2004; 43(3): 397-8.

79. Cohen MG, Webb J. Antihistone antibodies in rheumatoid arthritis and Felty's syndrome. Arthritis Rheum 1989; 32(10): 1319-24.

80. Vial T, Nicolas B, Descotes J. Drug-induced autoimmunity: experience of the French pharmacovigilance system. Toxicology 1997; 119(1): 23-7.

81. Biasi D, Caramaschi P, Carletto A, Bambara LM. Scleroderma and eosinophilic fasciitis in patients taking fosinopril. J Rheumatol 1997; 24(6): 1242.

82. Laroche M, Borg S, Lassoued S, De Lafontan B, Roché H. Joint pain with aromatase inhibitors: abnormal frequency of Sjögren's syndrome. J Rheumatol 2007; 34(11): 2259-63.

83. Noël B. Lupus erythematosus and other autoimmune diseases related to statin therapy: a systematic review. J Eur Acad Dermatol Venereol 2007; 21(1): 17-24.

84. Fauchais A, Iba Ba J, Maurage P, et al. [Polymyositis induced or associated with lipid-lowering drugs: five cases]. Rev Méd Interne 2004; 25(4): 294-8.

85. Chang C, Gershwin ME. Drugs and autoimmunity--a contemporary review and mechanistic approach. J Autoimmun 2010; 34(3): J266-75.

86. Ravnskov U. Glomerular, tubular and interstitial nephritis associated with non-steroidal antiinflammatory drugs. Evidence of a common mechanism. Br J Clin Pharmacol 1999; 47(2): 203-10.

87. Björnsson E, Talwalkar J, Treeprasertsuk S, et al. Drug-induced autoimmune hepatitis: clinical characteristics and prognosis. Hepatology 2010; 51(6): 2040-8

88. Savvas SP, Papakostas N, Giannaris M, Malaktari S, Koskinas J, Archimandritis AJ. Interferon alpha-induced hashimoto thyroiditis followed by transient graves disease in a patient with chronic HCV infection. South Med J 2010; 103(6): 585-8.

89. Garratty G. Immune hemolytic anemia caused by drugs. Expert Opin Drug Saf 2012; 11(4): 635-42.

90. Wittbrodt ET. Drugs and myasthenia gravis. An update. Arch Intern Med 1997; 157(4): 399-408.

91. Brenner S, Goldberg I. Drug-induced pemphigus. Clin Dermatol 2011; 29(4): 455-7.

92. Murad A, Connolly M, Tobin A. Rosuvastatin-induced pemphigoid. BMJ Case Rep 2012; 2012. pii:bcr1120115180.

93. Ruocco V, Sacerdoti G. Pemphigus and bullous pemphigoid due to drugs. Int J Dermatol 1991; 30(5): 307-12.

94. Law MR, Wald NJ, Rudnicka AR. Quantifying effect of statins on low density lipoprotein cholesterol, ischaemic heart disease, and stroke: systematic review and meta-analysis. BMJ 2003; 326(7404): 1423.

95. PROGRESS Collaborative Group. Randomised trial of a perindopril-based blood-pressure-lowering regimen among 6,105 individuals with previous stroke or transient ischaemic attack. Lancet 2001; 358(9287): 1033-41

96. MacMahon S, Peto R, Cutler J, et al. Blood pressure, stroke, and coronary heart disease. Part 1, Prolonged differences in blood pressure: prospective observational studies corrected for the regression dilution bias. Lancet 1990; 335(8692): 765-74.

97. Collins R, Peto R, MacMahon S, et al. Blood pressure, stroke, and coronary heart disease. Part 2, Short-term reductions in blood pressure: overview of randomised drug trials in their epidemiological context. Lancet 1990; 335(8693): 827-38. 
98. Antithrombotic Trialists' Collaboration. Collaborative meta-analysis of randomised trials of antiplatelet therapy for prevention of death, myocardial infarction, and stroke in high risk patients. BMJ 2002; 324(7329): 71-86.

99. Antiplatelet Trialists' Collaboration. Collaborative overview of randomised trials of antiplatelet therapy--I: Prevention of death, myocardial infarction, and stroke by prolonged antiplatelet therapy in various categories of patients. BMJ 1994; 308(6921): 81-106.

100. IMShealth.com. [internet] Parsippany, NJ: IMS Institute for Healthcare Informatics, c2011 [updated 2012 February 17; cited 2012 July 3]. Available from: http://www.imshealth.com/ims/Global/Content/Insights/ IMSInstituteforHealthcarelnformatics/IHII_Medicines_in_U.S_Report_2011.pdf.

101. Baigent C, Keech A, Kearney PM, et al. Efficacy and safety of cholesterol-lowering treatment: prospective meta-analysis of data from 90,056 participants in 14 randomised trials of statins. Lancet 2005; 366(9493): 1267-78.

102. Boekholdt SM, Arsenault B, Mora S, et al. Association of LDL cholesterol, non-HDL cholesterol, and apolipoprotein B levels with risk of cardiovascular events among patients treated with statins: a meta-analysis. JAMA 2012; 307(12): 1302-9

103. Endo A, Kuroda M, Tsujita Y. ML-236A, ML-236B, and ML-236C, new inhibitors of cholesterogenesis produced by Penicillium citrinium. J Antibiot 1976; 29(12): 1346-8.

104. Endo A. A gift from nature: the birth of the statins. Nat Med 2008; 14(10): 1050-2.

105. Goldstein JL, Brown MS. Familial hypercholesterolemia: identification of a defect in the regulation of 3-hydroxy-3-methylglutaryl coenzyme A reductase activity associated with overproduction of cholesterol. Proc Natl Acad Sci U S A 1973; 70(10): 2804-8.

106. Brown MS, Faust JR, Goldstein JL, Kaneko I, Endo A. Induction of 3-hydroxy-3-methylglutaryl coenzyme A reductase activity in human fibroblasts incubated with compactin (ML-236B), a competitive inhibitor of the reductase. J Biol Chem 1978; 253(4): 1121-8.

107. Corsini A, Maggi FM, Catapano AL. Pharmacology of competitive inhibitors of HMG-CoA reductase. Pharmacol Res 1995; 31(1): 9-27.

108. Brown MS, Goldstein JL. A receptor-mediated pathway for cholesterol homeostasis. Science 1986; 232(4746): 34-47.

109. Steffens S, Mach F. Drug insight: Immunomodulatory effects of statins--potential benefits for renal patients? Nat Clin Prac Nephrol 2006; 2(7): 378-87.

110. Glomset JA, Farnsworth CC. Role of protein modification reactions in programming interactions between ras-related GTPases and cell membranes. Annu Rev Cell Biol 1994; 10: 181-205.

111. Waldman A, Kritharides L. The pleiotropic effects of HMG-CoA reductase inhibitors: their role in osteoporosis and dementia. Drugs 2003; 63(2): 139-52.

112. Wennerberg K, Rossman K, Der C. The Ras superfamily at a glance. J Cell Sci 2005; 118(5): 843-6.

113. Veillard NR, Mach F. Statins: the new aspirin? Cell Mol Life Sci 2002; 59(11): 1771-86.

114. Laufs U, La Fata V, Plutzky J, Liao JK. Upregulation of endothelial nitric oxide synthase by HMG CoA reductase inhibitors. Circulation 1998; 97(12): 1129-35.

115. Dimmeler S, Fleming I, Fisslthaler B, Hermann C, Busse R, Zeiher AM. Activation of nitric oxide synthase in endothelial cells by Akt-dependent phosphorylation. Nature 1999; 399(6736): 601-5

116. Kimura T, Mogi C, Tomura $\mathrm{H}$, et al. Induction of scavenger receptor class $\mathrm{B}$ type I is critical for simvastatin enhancement of high-density lipoprotein-induced anti-inflammatory actions in endothelial cells. $J$ Immunol 2008; 181(10): 7332-40.

117. Kunieda Y, Nakagawa K, Nishimura H, et al. HMG CoA reductase inhibitor suppresses the expression of tissue factor and plasminogen activator inhibitor-1 induced by angiotensin II in cultured rat aortic endothelial cells. Thromb Res 2003; 110(4): 227-34.

118. Ohkita M, Sugii M, KaY, et al. Differential effects of different statins on endothelin-1 gene expression and endothelial NOS phosphorylation in porcine aortic endothelial cells. Exp Biol Med 2006; 231(6): 772-6.

119. Hernández Perera O, Pérez Sala D, Navarro Antolín J, Sánchez Pascuala R, Hernández G, Díaz C, Lamas S. Effects of the 3-hydroxy-3-methylglutaryl-CoA reductase inhibitors, atorvastatin and simvastatin, on the expression of endothelin-1 and endothelial nitric oxide synthase in vascular endothelial cells. J Clin Invest 1998; 101(12): 2711-9. 
120. Nishimura T, Vaszar L, Faul J, et al. Simvastatin rescues rats from fatal pulmonary hypertension by inducing apoptosis of neointimal smooth muscle cells. Circulation 2003; 108(13): 1640-5.

121. Erl W, Hristov M, Neureuter M, Yan Z, Hansson G, Weber P. HMG-CoA reductase inhibitors induce apoptosis in neointima-derived vascular smooth muscle cells. Atherosclerosis 2003; 169(2): 251-8.

122. Schäfer A, Fraccarollo D, Eigenthaler M, et al. Rosuvastatin reduces platelet activation in heart failure: role of NO bioavailability. Arterioscler Thromb Vasc Biol 2005; 25(5): 1071-7.

123. Tailor A, Lefer DJ, Granger DN. HMG-CoA reductase inhibitor attenuates platelet adhesion in intestinal venules of hypercholesterolemic mice. Am J Physiol Heart Circ Physiol 2004; 286(4): H1402-7.

124. Casani L, Sanchez Gomez S, Vilahur G, Badimon L. Pravastatin reduces thrombogenicity by mechanisms beyond plasma cholesterol lowering. Thromb Haemost 2005; 94(5): 1035-41.

125. Squizzato A, Romualdi E, Ageno W. Why should statins prevent venous thromboembolism? A systematic literature search and a call for action. J Thromb Haemost 2006; 4(9): 1925-7.

126. Tang D, Park H, Georgescu S, Sebti S, Hamilton A, Galper J. Simvastatin potentiates tumor necrosis factor alpha-mediated apoptosis of human vascular endothelial cells via the inhibition of the geranylgeranylation of RhoA. Life Sci 2006; 79(15): 1484-92.

127. Rezaie-Majd A, Maca T, Bucek RA, et al. Simvastatin reduces expression of cytokines interleukin-6, interleukin-8, and monocyte chemoattractant protein-1 in circulating monocytes from hypercholesterolemic patients. Arterioscler Thromb Vasc Biol 2002; 22(7): 1194-9.

128. Inoue I, Goto S, Mizotani K, et al. Lipophilic HMG-CoA reductase inhibitor has an anti-inflammatory effect: reduction of MRNA levels for interleukin-1beta, interleukin-6, cyclooxygenase-2, and p22phox by regulation of peroxisome proliferator-activated receptor alpha (PPARalpha) in primary endothelial cells. Life Sci 2000; 67(8): 863-76.

129. Tanimoto A, Murata Y, Wang K, Tsutsui M, Kohno K, Sasaguri Y. Monocyte chemoattractant protein-1 expression is enhanced by granulocyte-macrophage colony-stimulating factor via Jak2-Stat5 signaling and inhibited by atorvastatin in human monocytic U937 cells. J Biol Chem 2008; 283(8): 4643-51.

130. Veillard N, Braunersreuther V, Arnaud C, Burger F, Pelli G, Steffens S, Mach F. Simvastatin modulates chemokine and chemokine receptor expression by geranylgeranyl isoprenoid pathway in human endothelial cells and macrophages. Atherosclerosis 2006; 188(1): 51-8.

131. Sundararaj K, Samuvel D, Li Y, et al. Simvastatin suppresses LPS-induced MMP-1 expression in U937 mononuclear cells by inhibiting protein isoprenylation-mediated ERK activation. J Leukoc Biol 2008; 84(4): 1120-9.

132. Fujimoto S, Hartung D, Ohshima S, et al. Molecular imaging of matrix metalloproteinase in atherosclerotic lesions: resolution with dietary modification and statin therapy. J Am Coll Cardiol 2008; 52(23): 1847-57.

133. Wensley F, Gao P, Burgess S, et al. Association between C reactive protein and coronary heart disease: mendelian randomisation analysis based on individual participant data. BMJ 2011; 342: d548.

134. Ridker PM, Danielson E, Fonseca FA, et al. Rosuvastatin to prevent vascular events in men and women with elevated C-reactive protein. N Engl J Med 2008; 359(21): 2195-207.

135. Crouse J, Raichlen J, Riley W, et al. Effect of rosuvastatin on progression of carotid intima-media thickness in low-risk individuals with subclinical atherosclerosis: the METEOR Trial. JAMA 2007; 297(12): 1344-53.

136. Albert MA, Danielson E, Rifai N, Ridker PM, PRINCE Investigators. Effect of statin therapy on C-reactive protein levels: the pravastatin inflammation/CRP evaluation (PRINCE): a randomized trial and cohort study. JAMA 2001; 286(1): 64-70.

137. Heart Protection Study Collaborative Group, Jonathan E, Derrick B, et al. C-reactive protein concentration and the vascular benefits of statin therapy: an analysis of 20,536 patients in the Heart Protection Study. Lancet 2011; 377(9764): 469-76.

138. Katznelson S, Wilkinson AH, Kobashigawa JA, et al. The effect of pravastatin on acute rejection after kidney transplantation--a pilot study. Transplantation 1996; 61(10): 1469-74.

139. Kobashigawa JA, Katznelson S, Laks H, et al. Effect of pravastatin on outcomes after cardiac transplantation. N Engl J Med 1995; 333(10): 621-7.

140. Tuncer M, Süleymanlar G, Ersoy FF, Yakupo lu G. Comparison of the effects of simvastatin and pravastatin on acute rejection episodes in renal transplant patients. Transplant Proc 2000; 32(3): 622-5. 
141. Wenke K, Meiser B, Thiery J, et al. Simvastatin reduces graft vessel disease and mortality after heart transplantation: a four-year randomized trial. Circulation 1997; 96(5): 1398-402.

142. Kwak B, Mulhaupt F, Myit S, Mach F. Statins as a newly recognized type of immunomodulator. Nat Med 2000; 6(12): 1399-402.

143. Sadeghi MM, Tiglio A, Sadigh K, O'Donnell L, Collinge M, Pardi R, Bender JR. Inhibition of interferon-gamma-mediated microvascular endothelial cell major histocompatibility complex class II gene activation by HMG-CoA reductase inhibitors. Transplantation 2001; 71(9): 1262-8.

144. Shimada K, Miyauchi K, Daida $H$. Early intervention with atorvastatin modulates $T H 1 / T H 2$ imbalance in patients with acute coronary syndrome: from bedside to bench. Circulation 2004; 109(18): e213-4.

145. Gegg M, Harry R, Hankey D, et al. Suppression of autoimmune retinal disease by lovastatin does not require Th2 cytokine induction. J Immunol 2005; 174(4): 2327-35.

146. Mausner-Fainberg K, Luboshits G, Mor A, Maysel-Auslender S, Rubinstein A, Keren G, George J. The effect of HMG-CoA reductase inhibitors on naturally occurring CD4+CD25+ T cells. Atherosclerosis 2008; 197(2): 829-39.

147. Mira E, León B, Barber D, et al. Statins induce regulatory T cell recruitment via a CCL1 dependent pathway. J Immunol 2008; 181(5): 3524-34.

148. Kagami S, Owada $\mathrm{T}$, Kanari $\mathrm{H}$, et al. Protein geranylgeranylation regulates the balance between Th17 cells and Foxp3 regulatory T cells. Int Immunol 2009; 21(6): 679-89.

149. Wagner A, Gebauer M, GÃfÂA $1 / 4$ ldenzoph B, Hecker M. 3-hydroxy-3-methylglutaryl coenzyme A reductase-independent inhibition of CD40 expression by atorvastatin in human endothelial cells. Arterioscler Thromb Vasc Biol 2002; 22(11): 1784-9

150. Mulhaupt F, Matter CM, Kwak BR, et al. Statins (HMG-CoA reductase inhibitors) reduce CD40 expression in human vascular cells. Cardiovasc Res 2003; 59(3): 755-66.

151. Ferrara D, Liu X, Espinola R, Meroni P, Abukhalaf I, Harris EN, Pierangeli S. Inhibition of the thrombogenic and inflammatory properties of antiphospholipid antibodies by fluvastatin in an in vivo animal model. Arthritis Rheum 2003; 48(11): 3272-9

152. Kuwana M, Kaburaki J, Okazaki Y, Yasuoka H, Kawakami Y, Ikeda Y. Increase in circulating endothelial precursors by atorvastatin in patients with systemic sclerosis. Arthritis Rheum 2006; 54(6): 1946-51.

153. López Pedrera C, Ruiz Limón P, Aguirre MÃ, et al. Global effects of fluvastatin on the prothrombotic status of patients with antiphospholipid syndrome. Ann Rheum Dis 2011; 70(4): 675-82

154. Dormuth CR, Hemmelgarn BR, Paterson JM, et al. Use of high potency statins and rates of admission for acute kidney injury: multicenter, retrospective observational analysis of administrative databases. BMJ 2013; 346: f880.

155. Alsheikh Ali A, Ambrose M, Kuvin J, Karas R. The safety of rosuvastatin as used in common clinical practice: a postmarketing analysis. Circulation 2005; 111(23): 3051-7.

156. Jacobson T. Toward "pain-free" statin prescribing: clinical algorithm for diagnosis and management of myalgia. Mayo Clin Proc 2008; 83(6): 687-700.

157. Golomb BA, Evans MA. Statin adverse effects : a review of the literature and evidence for a mitochondrial mechanism. Am J Cardiovasc Drugs 2008; 8(6): 373-418.

158. Haroon M, Devlin J. A case of ANCA-associated systemic vasculitis induced by atorvastatin. Clin Rheumatol 2008; 27 Suppl 2: S75-7.

159. Purvin V, Kawasaki A, Smith K, Kesler A. Statin-associated myasthenia gravis: report of 4 cases and review of the literature. Medicine 2006; 85(2): 82-5.

160. Rudski L, Rabinovitch MA, Danoff D. Systemic immune reactions to HMG-CoA reductase inhibitors. Report of 4 cases and review of the literature. Medicine 1998; 77(6): 378-83.

161. Stoebner PE, Michot C, Ligeron C, Durand L, Meynadier J, Meunier L. Simvastatin-induced lichen planus pemphigoides. Ann Dermatol Venereol 2003; 130(2 Pt 1): 187-90.

162. Moulis G, Bene J, Sommet A, Sailler L, Lapeyre-Mestre M, Montastruc JL, French Association of PharmacoVigilance Centres. Statin-induced lupus: a case/non-case study in a nationwide pharmacovigilance database. Lupus 2012; 21(8): 885-9.

163. Russo MW, Scobey M, Bonkovsky HL. Drug-induced liver injury associated with statins. Semin Liver Dis 2009; 29(4): 412-22 
164. Rajabally YA, Varakantam V, Abbott RJ. Disorder resembling Guillain-Barre syndrome on initiation of statin therapy. Muscle Nerve 2004; 30(5): 663-6.

165. Knapp AC, Huang J, Starling G, Kiener PA. Inhibitors of HMG-CoA reductase sensitize human smooth muscle cells to Fas-ligand and cytokine-induced cell death. Atherosclerosis 2000; 152(1): 217-27.

166. Gurevich V, Shovman O, Slutzky L, Meroni P, Shoenfeld Y. Statins and autoimmune diseases. Autoimmun Rev 2005; 4(3): 123-9

167. Chow OA, von Kockritz-Blickwede M, Bright AT, et al. Statins enhance formation of phagocyte extracellular traps. Cell Host Microbe 2010; 8(5): 445-54.

168. Gupta AK, Joshi MB, Philippova M, Erne P, Hasler P, Hahn S, Resink TJ. Activated endothelial cells induce neutrophil extracellular traps and are susceptible to NETosis-mediated cell death. FEBS Lett 2010; 584(14): 3193-7.

169. Lin AM, Rubin CJ, Khandpur R, et al. Mast cells and neutrophils release IL-17 through extracellular trap formation in psoriasis. J Immunol 2011; 187(1): 490-500.

170. Kessenbrock K, Krumbholz M, Schonermarck U, et al. Netting neutrophils in autoimmune small-vessel vasculitis. Nat Med 2009; 15(6): 623-5

171. Leffler J, Martin M, Gullstrand B, et al. Neutrophil extracellular traps that are not degraded in systemic lupus erythematosus activate complement exacerbating the disease. J Immunol 2012; 188(7): 3522-31.

172. Villanueva E, Yalavarthi S, Berthier CC, et al. Netting neutrophils induce endothelial damage, infiltrate tissues, and expose immunostimulatory molecules in systemic lupus erythematosus. J Immunol 2011; 187(1): 538-52.

173. Hakkim A, Furnrohr BG, Amann K, et al. Impairment of neutrophil extracellular trap degradation is associated with lupus nephritis. Proc Natl Acad Sci U S A 2010; 107(21): 9813-8.

174. Matchar D, McCrory D, Orlando L, et al. Systematic review: comparative effectiveness of angiotensin-converting enzyme inhibitors and angiotensin II receptor blockers for treating essential hypertension. Ann Intern Med 2008; 148(1): 16-29.

175. Mielniczuk L, Stevenson L. Angiotensin-converting enzyme inhibitors and angiotensin II type I receptor blockers in the management of congestive heart failure patients: what have we learned from recent clinical trials? Curr Opin Cardiol 2005; 20(4): 250-5.

176. Young J, Dunlap M, Pfeffer M, et al. Mortality and morbidity reduction with Candesartan in patients with chronic heart failure and left ventricular systolic dysfunction: results of the CHARM low-left ventricular ejection fraction trials. Circulation 2004; 110(17): 2618-26.

177. Singh V. Review: ACE-Is or ARBs reduce adverse CV outcomes regardless of baseline systolic blood pressure. Ann Intern Med 2012; 157(2): JC1-8.

178. Lewis EJ, Hunsicker LG, Bain RP, Rohde RD. The effect of angiotensin-converting-enzyme inhibition on diabetic nephropathy. The Collaborative Study Group. N Engl J Med 1993; 329(20): 1456-62.

179. Imai E, Chan JCN, Ito S, Yamasaki T, Kobayashi F, Haneda M, Makino H. Effects of olmesartan on renal and cardiovascular outcomes in type 2 diabetes with overt nephropathy: a multicentre, randomised, placebo-controlled study. Diabetologia 2011; 54(12): 2978-86.

180. Skegss LT, Marsh WH, Kahn JR, Shumway NP. The existence of two forms of hypertensin. J Exp Med 1954; 99(3): 275-82

181. Skeggs LT, Marsh WH, Kahn JR, Shumway NP. The purification of hypertensin I. J Exp Med 1954; 100(4): 363-70.

182. Skeggs LT, Kahn JR, Shumway NP. The preparation and function of the hypertensin-converting enzyme. J Exp Med 1956; 103(3): 295-9.

183. $\mathrm{Ng} \mathrm{KK}$, Vane JR. Fate of angiotensin I in the circulation. Nature 1968; 218(5137): 144-50.

184. Vane JR. The release and fate of vaso-active hormones in the circulation. Br J Pharmacol 1969; 35(2): $209-42$.

185. Ferreira SH. A Bradykinin-potentiating factor (BPF) present in the venom of Bothrops Jararca. $\mathrm{Br} J$ Pharmacol Chemother 1965; 24: 163-9.

186. Cushman DW, Ondetti MA. Design of angiotensin converting enzyme inhibitors. Nat Med 1999; 5(10): 1110-3.

187. Ondetti MA, Rubin B, Cushman DW. Design of specific inhibitors of angiotensin-converting enzyme: new class of orally active antihypertensive agents. Science 1977; 196(4288): 441-4. 
188. Food Drug Administration. Captopril approved for hypertension. FDA Drug Bull 1981; 11(2): 10-1.

189. Timmermans PB, Duncia JV, Carini DJ, Chiu AT, Wong PC, Wexler RR, Smith RD. Discovery of losartan, the first angiotensin II receptor antagonist. J Hum Hypertens 1995; 9 Suppl 5: S3-18.

190. Erdös EG, Skidgel RA. Human angiotensin I converting enzyme: unusual substrate specificity and the distribution. Hypertension 1986; 8(suppl 1): 1-34,1-37.

191. Ellis ML, Patterson JH. A new class of antihypertensive therapy: angiotensin II receptor antagonists. Pharmacotherapy 1996; 16(5): 849-60.

192. Alderman CP. Adverse effects of the angiotensin-converting enzyme inhibitors. Ann Pharmacother 1996; 30(1): 55-61.

193. Acker CG, Greenberg A. Angioedema induced by the angiotensin II blocker losartan. N Engl J Med 1995; 333(23): 1572

194. Barreras A, Gurk Turner C. Angiotensin II receptor blockers. Proc (Bayl Univ Med Cent) 2003; 16(1): 123-6.

195. Sica D. Angiotensin-converting enzyme inhibitors side effects--physiologic and non-physiologic considerations. J Clin Hypertens (Greenwich) 2004; 6(7): 410-6.

196. Van Rijnsoever EW, Kwee-Zuiderwijk WJ, Feenstra J. Angioneurotic edema attributed to the use of losartan. Arch Intern Med 1998; 158(18): 2063-5.

197. Lacourciere $\mathrm{Y}$, Brunner $\mathrm{H}$, Irwin R, et al. Effects of modulators of the renin-angiotensin-aldosterone system on cough. Losartan Cough Study Group. J Hypertens 1994; 12(12): 1387-93.

198. Benz J, Oshrain C, Henry D, Avery C, Chiang YT, Gatlin M. Valsartan, a new angiotensin II receptor antagonist: a double-blind study comparing the incidence of cough with lisinopril and hydrochlorothiazide. J Clin Pharmacol 1997; 37(2): 101-7.

199. Lacourciere $Y$. The incidence of cough: a comparison of lisinopril, placebo and telmisartan, a novel angiotensin II antagonist. Telmisartan Cough Study Group. Int J Clin Pract 1999; 53(2): 99-103.

200. Goa KL, Wagstaff AJ. Losartan potassium: a review of its pharmacology, clinical efficacy and tolerability in the management of hypertension. Drugs 1996; 51(5): 820-45

201. McClellan KJ, Markham A. Telmisartan. Drugs 1998; 56(6): 1039,44; discussion 1045-6.

202. Sever P. Candesartan cilexetil: a new, long-acting, effective angiotensin II type 1 receptor blocker. J Hum Hypertens 1997; 11 Suppl 2: S91-5.

203. Siragy $\mathrm{H}$. A current evaluation of the safety of angiotensin receptor blockers and direct renin inhibitors. Vasc Health Risk Manag 2011; 7: 297-313.

204. Burnier M, Brunner HR. Angiotensin II receptor antagonists. Lancet 2000; 355(9204): 637-45.

205. Mazzolai L, Burnier M. Comparative safety and tolerability of angiotensin II receptor antagonists. Drug Saf 1999; 21(1): 23-33.

206. Sipahi I, Debanne S, Rowland D, Simon D, Fang J. Angiotensin-receptor blockade and risk of cancer: meta-analysis of randomised controlled trials. Lancet Oncol 2010; 11(7): 627-36.

207. Deshayes F, Nahmias C. Angiotensin receptors: a new role in cancer? Trends Endocrinol Metab 2005; 16(7): 293-9

208. Soto-Pantoja DR, Menon J, Gallagher PE, Tallant EA. Angiotensin-(1-7) inhibits tumor angiogenesis in human lung cancer xenografts with a reduction in vascular endothelial growth factor. Mol Cancer Ther 2009; 8(6): 1676-83.

209. Walther T, Menrad A, Orzechowski HD, Siemeister G, Paul M, Schirner M. Differential regulation of in vivo angiogenesis by angiotensin II receptors. FASEB J 2003; 17(14): 2061-7.

210. Volpert OV, Ward WF, Lingen MW, et al. Captopril inhibits angiogenesis and slows the growth of experimental tumors in rats. J Clin Invest 1996; 98(3): 671-9.

211. Small W, Molteni A, Kim YT, Taylor JM, Chen Z, Ward WF. Captopril modulates hormone receptor concentration and inhibits proliferation of human mammary ductal carcinoma cells in culture. Breast Cancer Res Treat 1997; 44(3): 217-24

212. Katayama S, Inaba M, Maruno Y, Omoto A, Itabashi A, Kawazu S, Ishii J. Effect of captopril or enalapril on renal prostaglandin E2. Prostaglandins 1989; 38(4): 401-11.

213. Goto M, Fujisawa M, Yamada A, Okabe T, Takaku F, Sasano M, Nishioka K. Spontaneous release of angiotensin converting enzyme and interleukin 1 beta from peripheral blood monocytes from patients with rheumatoid arthritis under a serum free condition. Ann Rheum Dis 1990; 49(3): 172-6. 
214. Walsh DA, Catravas J, Wharton J. Angiotensin converting enzyme in human synovium: increased stromal [(125)I]351A binding in rheumatoid arthritis. Ann Rheum Dis 2000; 59(2): 125-31.

215. Constantinescu CS, Goodman DB, Grossman RI, Mannon LJ, Cohen JA. Serum angiotensin-converting enzyme in multiple sclerosis. Arch Neurol 1997; 54(8): 1012-5.

216. Lovreci L, Risti S, Starcevi Cizmarevi N, Jazbec SS, Sepci J, Kapovi M, Peterlin B. Angiotensin-converting enzyme I/D gene polymorphism and risk of multiple sclerosis. Acta Neurol Scand 2006; 114(6): 374-7.

217. Shao J, Nangaku M, Miyata T, Inagi R, Yamada K, Kurokawa K, Fujita T. Imbalance of T-cell subsets in angiotensin II-infused hypertensive rats with kidney injury. Hypertension 2003; 42(1): 31-8.

218. Multicenter Captopril Study Group. Captopril reduces the risk of nephropathy in IDDM patients with microalbuminuria. The Microalbuminuria Captopril Study Group. Diabetologia 1996; 39(5): 587-93.

219. Herlitz H, Tarkowski A, Svalander C, Volkmann R, Westberg G. Beneficial effect of captopril on systemic lupus erythematosus-like disease in MRL Ipr/Ipr mice. Int Arch Allergy Appl Immunol 1988; 85(3): 272-7.

220. Herlitz H, Svalander C, Tarkowski A, Westberg G. Effect of captopril on murine systemic lupus erythematosus disease. J Hypertens Suppl 1988; 6(4): S684-6.

221. Godsel LM, Leon JS, Wang K, Fornek JL, Molteni A, Engman DM. Captopril prevents experimental autoimmune myocarditis. J Immunol 2003; 171(1): 346-52.

222. Constantinescu CS, Ventura E, Hilliard B, Rostami A. Effects of the angiotensin converting enzyme inhibitor captopril on experimental autoimmune encephalomyelitis. Immunopharmacol Immunotoxicol 1995; 17(3): 471-91.

223. Kallenberg CG, Hoorntje SJ, Smit AJ, Weening JJ, Donker AJ, Hoedemaeker PH, The TH. Antinuclear and antinative DNA antibodies during captopril treatment. Acta Med Scand 1982; 211(4): 297-300.

224. Reidenberg MM, Case DB, Drayer DE, Reis S, Lorenzo B. Development of antinuclear antibody in patients treated with high doses of captopril. Arthritis Rheum 1984; 27(5): 579-81.

225. Schwartz D, Pines A, Averbuch M, Levo Y. Enalapril-induced antinuclear antibodies. Lancet 1990; 336(8708): 187.

226. Sieber C, Grimm E, Follath F. Captopril and systemic lupus erythematosus syndrome. BMJ 1990; 301(6753): 669.

227. Ratliff NB, 3rd. Captopril induced lupus. J Rheumatol 2002; 29(8): 1807-8.

228. Ruocco V, Satriano RA, Guerrera V. "Two-step" pemphigus induction by ACE-inhibitors. Int J Dermatol 1992; 31(1): 33-6.

229. Ong CS, Cook N, Lee S. Drug-related pemphigus and angiotensin converting enzyme inhibitors. Australas J Dermatol 2000; 41(4): 242-6.

230. Parodi A, Cozzani E, Milesi G, Drosera M, Rebora A. Fosinopril as a possible pemphigus-inducing drug. Dermatology 2002; 204(2): 139-41.

231. Patterson CR, Davies MG. Pemphigus foliaceus: an adverse reaction to lisinopril. J Dermatolog Treat 2004; 15(1): 60-2.

232. Pelayo M, Vargas V, Gonzales A, Vallano A, Esteban R, Guardia J. Drug-induced lupus-like reaction and captopril. Ann Pharmacother 1993; 27(12): 1541-2

233. Patri P, Nigro A, Rebora A. Lupus erythematosus-like eruption from captopril. Acta Derm Venereol 1985; 65(5): 447-8.

234. Pinto GM, Lamarao P, Vale T. Captopril-induced pemphigus vegetans with Charcot-Leyden crystals. J Am Acad Dermatol 1992; 27(2 Pt 2): 281-4.

235. Vignes S, Paul C, Flageul B, Dubertret L. Ramipril-induced superficial pemphigus. Br J Dermatol 1996; 135(4): 657-8.

236. Moots RJ, Keeling PJ, Morgan SH. Adult Schonlein-Henoch purpura after enalapril. Lancet 1992; 340(8814): 304-5.

237. Kuechle MK, Hutton KP, Muller SA. Angiotensin-converting enzyme inhibitor-induced pemphigus: three case reports and literature review. Mayo Clin Proc 1994; 69(12): 1166-71.

238. Kaplan RP, Potter TS, Fox JN. Drug-induced pemphigus related to angiotensin-converting enzyme inhibitors. J Am Acad Dermatol 1992; 26(2 Pt 2): 364-6.

239. Gupta S, Gandhi NM, Ferguson J. Cutaneous vasculitis secondary to ramipril. J Drugs Dermatol 2004; 3(1): 81-2. 
240. Goncalves R, Cortez Pinto H, Serejo F, Ramalho F. Adult Schonlein-Henoch purpura after enalapril. J Intern Med 1998; 244(4): 356-7.

241. Fernandez-Diaz ML, Herranz P, Suarez-Marrero MC, Borbujo J, Manzano R, Casado M. Subacute cutaneous lupus erythematosus associated with cilazapril. Lancet 1995; 345(8946): 398

242. Disdier P, Harle JR, Verrot D, Jouglard J, Weiller PJ. Adult Schonlein-Henoch purpura after lisinopril. Lancet 1992; 340(8825): 985.

243. Buzon E, Perez-Bernal AM, de la Pena F, Rios JJ, Camacho F. Pemphigus foliaceus associated with cilazapril. Acta Derm Venereol 1998; 78(3): 227

244. Butt A, Burge SM. Pemphigus vulgaris induced by captopril. Br J Dermatol 1995; 132(2): 315-6.

245. Bertin P, Kamdem J, Bonnet C, Arnaud M, Treves R. Captopril-induced lupus. Clin Exp Rheumatol 1993; 11(6): 695.

246. Bastiaens MT, Zwan NV, Verschueren GL, Stoof TJ, Nieboer C. Three cases of pemphigus vegetans: induction by enalapril--association with internal malignancy. Int J Dermatol 1994; 33(3): 168-71.

247. Bae YI, Yun SJ, Lee SC, Park GT, Lee JB. Pemphigus foliaceus induced by an angiotensin II receptor blocker. Clin Exp Dermatol 2008; 33(6): 721-3.

248. Coelho dos Santos JS, Menezes CA, Villani FN, Magalhaes LM, Scharfstein J, Gollob KJ, Dutra WO. Captopril increases the intensity of monocyte infection by Trypanosoma cruzi and induces human $T$ helper type 17 cells. Clin Exp Immunol 2010; 162(3): 528-36.

249. Tesmer L, Lundy S, Sarkar S, Fox D. Th17 cells in human disease. Immunol Rev 2008; 223: 87-113.

250. Odaka C, Mizuochi T. Angiotensin-converting enzyme inhibitor captopril prevents activation-induced apoptosis by interfering with T cell activation signals. Clin Exp Immunol 2000; 121(3): 515-22.

251. Deas O, Dumont C, Mollereau B, et al. Thiol-mediated inhibition of FAS and CD2 apoptotic signaling in activated human peripheral T cells. Int Immunol 1997; 9(1): 117-25.

252. Noël B, Panizzon R. Lupus-like syndrome associated with statin therapy. Dermatology 2004; 208(3): 276-7.

253. Noël B. Risks and benefits of statins in lupus erythematosus. Arch Intern Med 2004; 164(1): 107-8

254. Fadel F, El Karoui K, Knebelmann B. Anti-CTLA4 antibody-induced lupus nephritis. N Eng J Med 2009; 361(2): 211-2. 


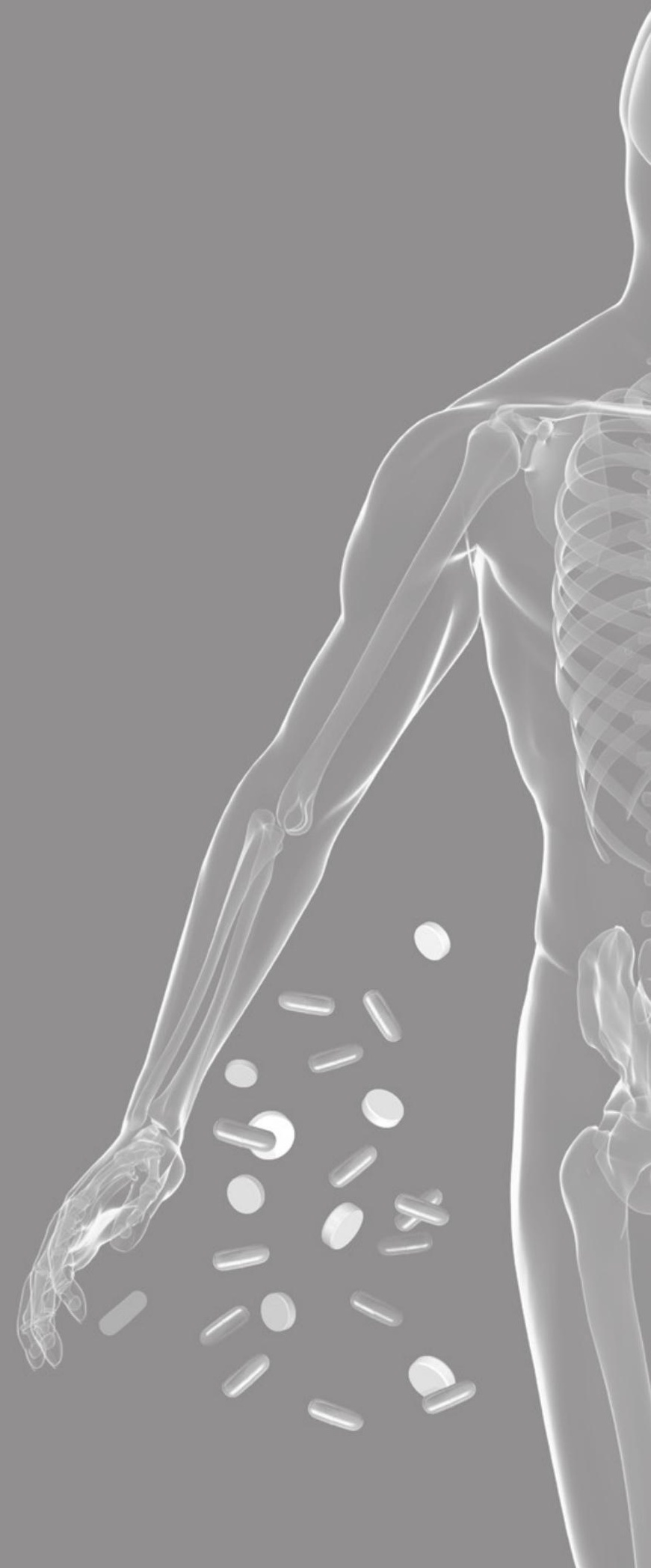




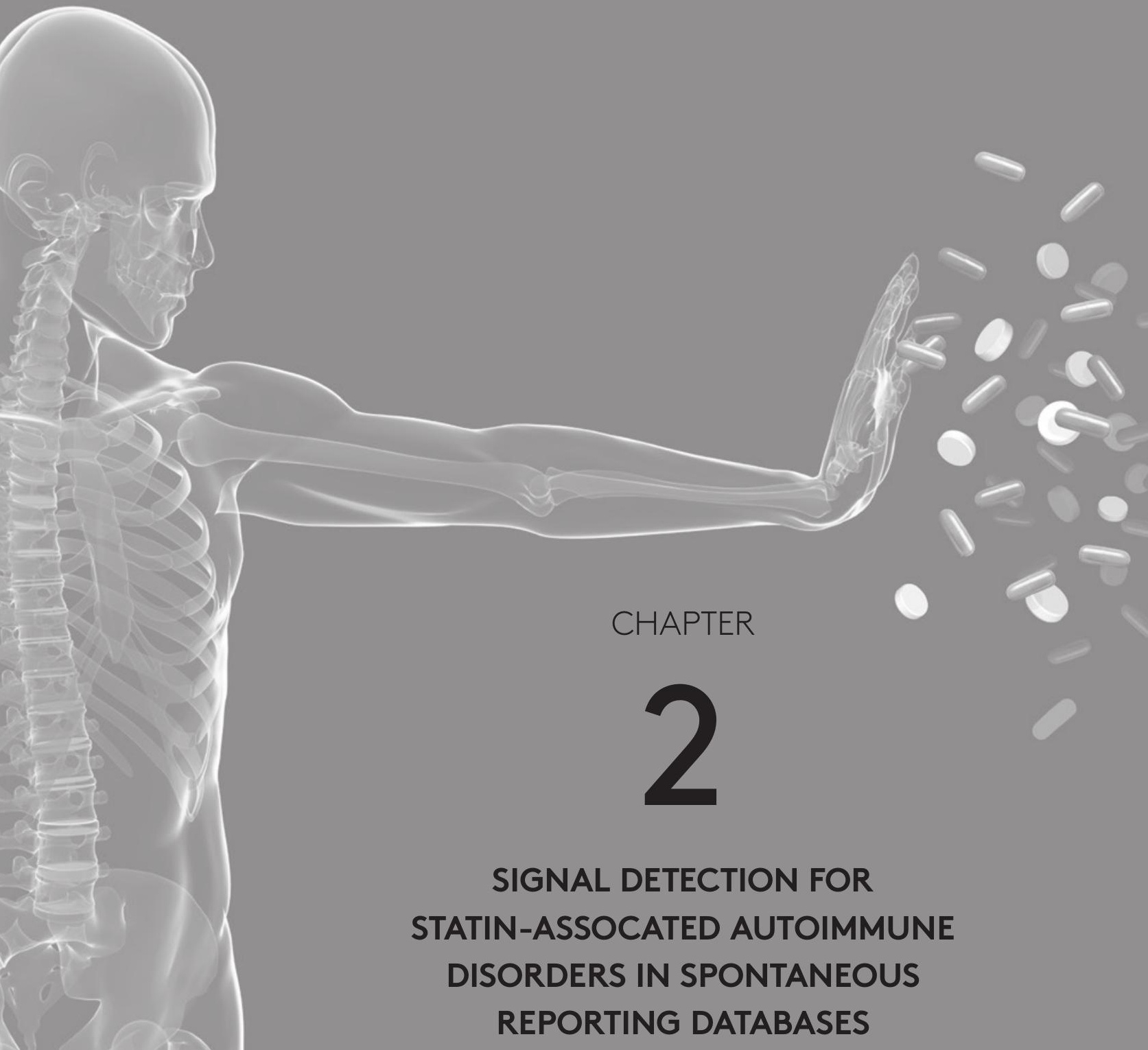




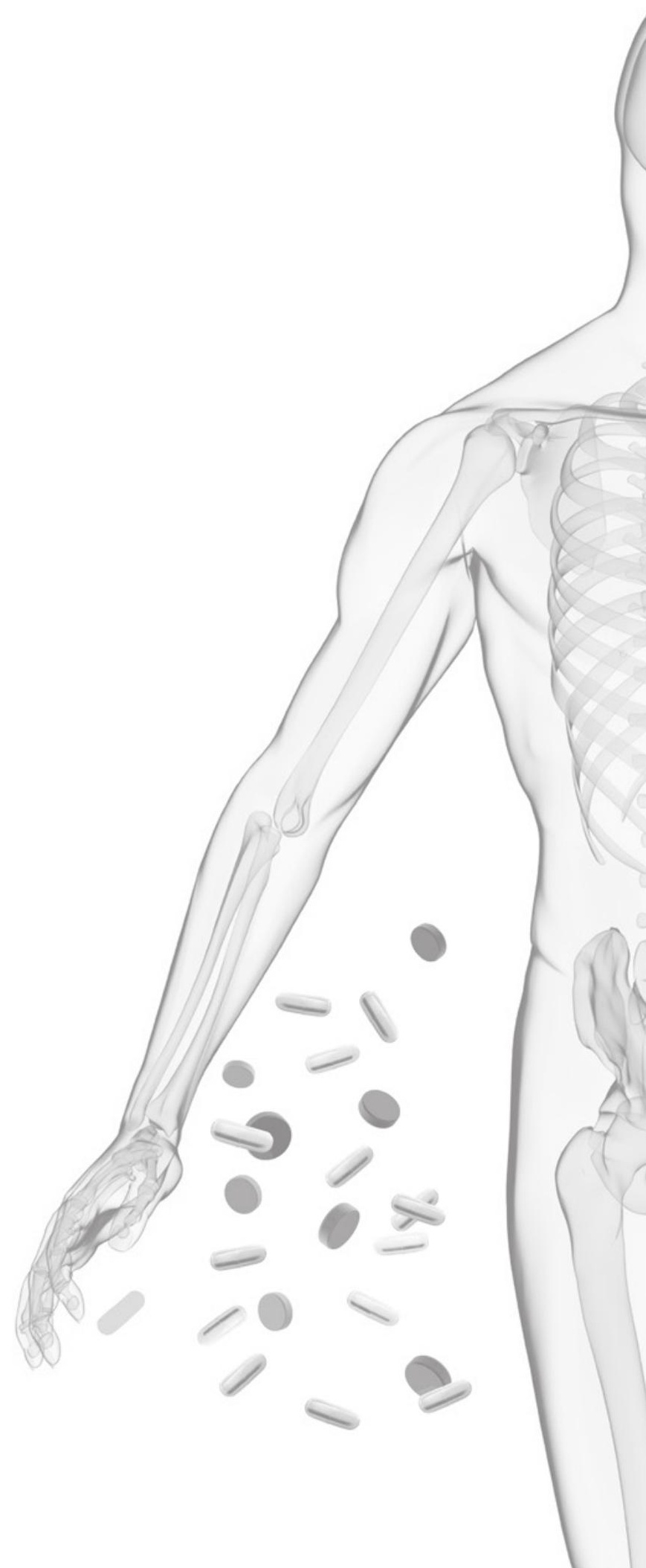




\section{CHAPTER}

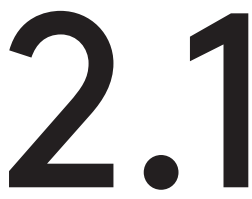

\section{ASSOCIATION BETWEEN STATIN USE AND LUPUS-LIKE SYNDROME USING SPONTANEOUS REPORTS}

H. J. I. de Jong, JW. Cohen Tervaert, S. R. F. Saldi, R. J. Vandebriel, P.C. Souverein, R. H. B. Meyboom, H. van Loveren, O. H. Klungel 


\section{ABSTRACT}

\section{Objective}

Several case reports of lupus-like syndrome suggest that statins could have triggered the development of this rare autoimmune disease. However, data on the association between statin use and lupus-like syndrome are scarce. We assessed whether there was an association between statin use and the occurrence of lupus-like syndrome.

\section{Methods}

A case/noncase study based on individual case safety reports listed in the World Health Organisation global individual case safety reports database (VigiBase) was conducted. According to World Health Organisation adverse reaction terminology, cases were defined as reports of lupus-like syndrome. Each case was matched with five non-cases by age, sex, and time of reporting. The use of statins was classified according to the Anatomical Therapeutic Chemical classification code system. Covariates, i.e., use of corticosteroids, immunosuppressive drugs, non-steroidal anti-inflammatory drugs, antidepressants, anti-epileptics, proton pump inhibitors and cardiovascular drugs, were determined. Multivariate logistic regression was used to calculate the reporting odds ratios with 95\% confidence intervals.

\section{Results}

We identified 3362 reports of lupus-like syndrome as cases and 27092 reports of other adverse drug reactions as non-cases. Statins were more frequently reported as suspected drug in cases (3.2\%) than in non-cases (1.5\%). After adjustment for several covariates, statins were associated with the reporting of lupus-like syndrome (reporting odds ratios 2.01; 95\% confidence intervals 1.61 to 2.51 ).

\section{Conclusions}

We found an association between reporting of statins and lupus-like syndrome. Further studies are needed to confirm this finding in more detail and establish causality. 


\section{INTRODUCTION}

Since the introduction of lovastatin in 1987, 3-hydroxy-3-methylglutaryl coenzyme A (HMG-CoA) reductase inhibitors, or statins, have been shown to be effective in reducing the risk of cardiovascular morbidity and mortality in patients with hyperlipidaemia, hypertension, or diabetes mellitus type II ${ }^{1-5}$. In addition to their cholesterol-lowering activity, several studies have shown that statins have anti-inflammatory and immunomodulatory properties. These properties may eventually lead to dysregulation of immune responses ${ }^{6-8}$. Hence, statins may facilitate the development of autoimmunity, eventually resulting in autoimmune diseases. An increasing number of case reports suggest that statins could trigger the development of lupus-like syndrome (Table 1) 9-20. Recently, two reviews have summarised the case reports of lupus-like syndrome in patients using statins 21,22. Based on these case reports alone, the incidence of statin-induced lupus-like syndrome cannot be established. We postulated that this possible statin-induced lupus-like syndrome is relevant in daily practice and may be reported to pharmacovigilance centres. The aim of our study was to assess the association between statin use and the occurrence of lupus-like syndrome by carrying out a case/non-case approach based on individual case safety reports (ICSRs) of adverse drug reactions (ADRs).

\section{METHODS}

\section{Study Population}

The association between the use of statins and lupus-like syndrome was evaluated using the database of the World Health Organisation Uppsala Monitoring Centre (WHO UMC), Sweden. The database (VigiBase) contains the global ICSRs of suspected adverse reactions to pharmaceutical products submitted through National Pharmacovigilance Centres by 90 countries around the world. In February 2009, this database contained more than 4.6 million ICSRs of suspected ADRs 23 . At the national level, ADRs are reported by health care professionals and in some countries by patients and pharmaceutical companies. Details about suspected ADRs such as age, sex, reporting date, country, nature of the ADR, suspected drugs, concomitantly used drugs, and interacting drugs are available in the VigiBase. ADRs are coded according to the WHO Adverse Reaction Terminology (WHO-ART) or the Medical Dictionary for Regulatory Activities 23 . The suspected drugs are classified according to the Anatomical Therapeutic Chemical classification. Information in these reports is not homogenous, at least with regard to origin, completeness of documentation, or the likelihood that the suspected drugs caused the adverse events 23 . 
Table 1 Overview case-reports of lupus-like syndrome

\begin{tabular}{|c|c|c|c|c|c|c|c|}
\hline Ref & Sex/Age & Diagnosis & $\begin{array}{l}\text { Exposure } \\
\text { statins }\end{array}$ & $\begin{array}{l}\text { Other } \\
\text { drugs }\end{array}$ & ANA $^{*}$ & $\begin{array}{l}\text { Anti- } \\
\text { DNA }\end{array}$ & $\begin{array}{l}\text { Anti- } \\
\text { histone }\end{array}$ \\
\hline 10 & $F / 64$ & $\begin{array}{l}\text { Lupus-like } \\
\text { syndrome }\end{array}$ & $\begin{array}{l}\text { Lovastatin } \\
20 \text { mg/day }\end{array}$ & - & Pos & Neg & Neg \\
\hline 10 & $\mathrm{M} / 40$ & $\mathrm{DIL}^{\dagger}$ & Lovastatin & - & Pos & Neg & Pos \\
\hline 11 & $\mathrm{~F} / 70$ & $\begin{array}{l}\text { Lupus-like } \\
\text { syndrome }\end{array}$ & Simvastatin 10 mg/day & - & Pos & Neg & Neg \\
\hline 12 & $M / 39$ & $\begin{array}{l}\text { Lupus-like } \\
\text { syndrome }\end{array}$ & Simvastatin 20 mg/day & - & Pos & Neg & Neg \\
\hline
\end{tabular}

\begin{tabular}{|c|c|c|c|c|c|c|c|}
\hline 14 & $\mathrm{M} / 79$ & DIL & Simvastatin & - & Pos & Neg & Pos \\
\hline 16 & $\mathrm{M} / 78$ & SLE $\ddagger$ & $\begin{array}{l}\text { Pravastatin } \\
40 \mathrm{mg} / \text { day }\end{array}$ & $\begin{array}{l}\text { Metoprolol } \\
\text { Nifedipine } \\
\text { Terazocin } \\
\text { Fluoxetine } \\
\text { Lorazepam } \\
\text { Cimetidine } \\
\text { Nitroglycerin }\end{array}$ & Pos & Pos & - \\
\hline 17 & $\mathrm{~F} / 67$ & $\begin{array}{l}\text { Lupus-like } \\
\text { syndrome, ARDS§ }\end{array}$ & $\begin{array}{l}\text { Fluvastatin } \\
20 \mathrm{mg} / \text { day }\end{array}$ & $\begin{array}{l}\text { Atenolol } \\
\text { Aspirin }\end{array}$ & Pos & Pos & Neg \\
\hline 9 & $\mathrm{M} / 42$ & DIL & Simvastatin 20 mg/day & $\begin{array}{l}\text { Metformin } \\
\text { Glipizide }\end{array}$ & Pos & Neg & Pos \\
\hline 13 & $M / 26$ & DIL & $\begin{array}{l}\text { Atorvastatin } \\
20 \mathrm{mg} / \text { day }\end{array}$ & - & Pos & Neg & Pos \\
\hline 19 & $F / 58$ & $\begin{array}{l}\text { Lupus-like } \\
\text { syndrome, } \\
\text { autoimmune } \\
\text { hepatitis }\end{array}$ & Atorvastatin & & Pos & - & - \\
\hline 20 & $M / 58$ & SCLE"I & Pravastatin & Atenolol & Pos & Neg & Neg \\
\hline 20 & $F / 53$ & SCLE & Simvastatin & Ibuprofen & Pos & Neg & Neg \\
\hline 15 & $M / 59$ & $\begin{array}{l}\text { Lupus-like } \\
\text { syndrome }\end{array}$ & $\begin{array}{l}\text { Simvastatin } \\
20 \mathrm{mg} / \text { day }\end{array}$ & - & Pos & Pos & $\mathrm{Neg}$ \\
\hline 18 & $F / 82$ & SCLE & Simvastatin & $\begin{array}{l}\text { Aspirin } \\
\text { Lansoprazole }\end{array}$ & Pos & Neg & $\mathrm{Neg}$ \\
\hline
\end{tabular}

*antinuclear antibodies, †drug-induced lupus, ‡systemic lupus erythematosus, §adult respiratory distress syndrome, "subacute cutane lupus erythematosus, "anti-nucleosome antibodies 


\begin{tabular}{|c|c|c|c|}
\hline $\begin{array}{l}\text { Other } \\
\text { antibodies }\end{array}$ & $\begin{array}{l}\text { Time to } \\
\text { onset }\end{array}$ & treatment & development \\
\hline & $2 \mathrm{yr}$ & - Prednisone 20 mg/day & - resolved in 6 wk \\
\hline & $2 \mathrm{yr}$ & - Prednisone 20 mg/day & - resolved in 6 wk \\
\hline & $18 \mathrm{mo}$ & - Prednisone 15 mg/day & - resolved in 2 wk \\
\hline & $4 \mathrm{yr}$ & - Nifedipine for Raynaud's symptoms & $\begin{array}{l}\text { - improved after } 2 \text { mo } \\
\text { - at } 5 \text { mo mild Raynaud's }\end{array}$ \\
\hline & $3 \mathrm{mo}$ & - Prednisone 40 mg/day & - resolved in 2 wk \\
\hline $\begin{array}{l}\text { - Pos cryoglobulin } \\
\text { - Pos anticardiolipin }\end{array}$ & $6 \mathrm{yr}$ & $\begin{array}{l}\text { - Prednisone } 50 \text { mg/day, } \\
\text { - Plaquenil }\end{array}$ & - disease active at $24 \mathrm{mo}$ \\
\hline
\end{tabular}

\begin{tabular}{|c|c|c|c|}
\hline & $1 w k$ & $\begin{array}{l}\text { - Methylprednisolone, } \\
\text { - Cyclophosphamide }\end{array}$ & - Died due to ARDS \\
\hline & $2 w k$ & - & - resolved in 2 wk \\
\hline & $1 \mathrm{yr}$ & - & - resolved in $11 \mathrm{mo}$ \\
\hline - Pos Nuc" & $7 \mathrm{mo}$ & $\begin{array}{l}\text { - Tacrolimus } 3 \mathrm{mg} / \mathrm{twice} \text { day, } \\
\text { - Mycophenolate mofetil } 1000 \mathrm{mg} / \mathrm{twice} \text { day, } \\
\text { - prednisolone } 10 \mathrm{mg} / \mathrm{day}\end{array}$ & - in remission after $12 \mathrm{mo}$ \\
\hline - Pos Ro/SSA & 6 wks & - topical corticosteroids & - improved in 6-12 wk \\
\hline \multirow[t]{2}{*}{ - Pos Ro/SSA } & $8 w k$ & - topical corticosteroids & - improved in 6-12 wk \\
\hline & $2 \mathrm{yr}$ & & - resolved in $12 \mathrm{mo}$ \\
\hline - Pos Ro/SSA & $3 \mathrm{mo}$ & $\begin{array}{l}\text { - Hydroxychloroquine, } \\
\text { - Prednisolone, } \\
\text { - topical corticosteroids }\end{array}$ & - resolved in $2 \mathrm{wk}$ \\
\hline
\end{tabular}




\section{Design}

A case/non-case approach was used to evaluate the association between the use of statins and lupus-like syndrome. In the VigiBase, cases of lupus-like syndrome were defined as all available ADR reports containing the following WHO-ART adverse reaction terms: "lupus erythematosus (LE) rash," "LE discoid," "LE-like butterfly rash," "LE-like discoid rash," "LE systemic," "Disseminated lupus erythematosus (DLE)," "DLE," "LE arthritis," "LE type reaction," "Lupus erythematosus systemic (SLE)," "SLE-like symptoms," "Systemic lupus erythematosus syndrome," and "hydralazine LE type reaction" (Table 2) ${ }^{23}$. These WHO-ART adverse reaction terms were attached to the original report by the national pharmacovigilance centre or the reporter of the ADR. Reports were only included when data on sex and age were available. Since we are interested in incident cases of lupus-like syndrome, ADR reports with the preferred terms "LE syndrome aggravated" were excluded from the study. Each case was matched with five non-cases by age, sex and calendar year of reporting. Non-cases were considered as reports containing other adverse reaction terms than lupus-like syndrome, including other autoimmune drug reactions.

\section{Definition of Exposure}

Exposure to statins was defined as reports in which the reporter suspected that a prescribed statin was a causal factor in the development of lupus-like syndrome. The Anatomical Therapeutic Chemical codes for statins were C10AA (HMG-CoA reductase inhibitors), C10BA (HMG-CoA reductase inhibitors in combination with other lipid modifying agents), and C10BX (HMG-CoA reductase inhibitors and other combinations) 24 .

\section{Covariates}

Concomitant medication, i.e., use of anti-arrhythmic drugs, anti-hypertensive and anti-diabetic agents, non-steroidal anti-inflammatory drugs, corticosteroids, disease modifying anti-rheumatic drugs, antidepressants, anti-epileptics and proton pump inhibitors, associated with lupus-like syndrome were considered as covariates 25 .

\section{Statistical Analysis}

Characteristics of the cases and non-cases were analysed, using Student's t test or Chi-square test. Means and standard deviations or percentages were obtained for continuous and categorical variables, respectively. The strength of the association between reporting of statins and lupus-like syndrome was assessed using logistic regression analysis and expressed as reporting odds ratios (ROR) accompanied with $95 \%$ confidence intervals $(\mathrm{Cl})$. The ROR provides an estimate of the extent to which lupus-like syndrome is reported in association with statins as suspected drug relative to reports of lupus-like syndrome in which other drugs were the suspected 
Table 2 Terms for coding lupus-like syndrome defined by the preferred terms of the WHO Adverse Reaction Terminology (WHO-ART) 2007

\begin{tabular}{lllll} 
High level term & Recno* & Preferred term & Seq $^{\dagger}$ & Included term \\
\hline LE Syndrome & 0080 & LE Rash & & \\
\hline & & & 004 & LE-like butterfly rash \\
\hline & & 005 & LE-like discoid rash \\
\hline & & 003 & Lupus erythematosus discoid \\
\hline
\end{tabular}

\begin{tabular}{llll}
\hline 0081 & LE Systemic $\neq, \S, \|$ & 005 & Disseminated lupus erythematosus \\
\hline & 003 & DLE \\
\hline 004 & Hydralazine LE type reaction \\
\hline 006 & LE arthritis \\
\hline 007 & LE type reaction \\
\hline 012 & Lupus encephalitis \\
\hline 008 & Lupus erythematosus systemic \\
\hline 009 & SLE-like symptoms \\
\hline 010 & $\begin{array}{l}\text { Systemic lupus erythematosus } \\
\text { syndrome }\end{array}$ \\
\hline &
\end{tabular}

0082 LE test abnormal§,॥ $004 \quad$ LE cells present

\begin{tabular}{|c|c|c|c|}
\hline 1082 & $\begin{array}{l}\text { Antinuclear factor } \\
\text { test positive§,॥ }\end{array}$ & 004 & Anti-dsDNA \\
\hline & & 003 & Antinuclear factor \\
\hline \multicolumn{4}{|c|}{$\begin{array}{l}\text { *record number, }{ }^{\dagger} \text { sequence, } \neq \text { sensitivity analysis } 2 \text { with the preferred term (and included term), } \\
\text { §sensitivity analysis } 3 \text { with the preferred term (and included term), "sensitivity analysis } 4 \text { with preferred } \\
\text { term (and included term), "anti-double-stranded DNA }\end{array}$} \\
\hline
\end{tabular}

agent. Covariates that acted as confounders were included in the model if each of them induced a change of crude $\beta$ estimates of the exposure-outcome association of at least $10 \% 26$. All tests were 2 -sided with a rejection of the null hypothesis at a $p$ value of less than 0.05 . All the analyses were conducted using SPSS 16.0 statistical software (SPSS Inc., Chicago, Illinois, USA). 


\section{Sensitivity Analysis}

Since the reporters may not always be aware of statin-induced lupus-like syndrome, we performed a sensitivity analysis where we expanded our definition of statin use to reports where statins were classified as suspected or concomitant drug for an ADR. To explore the influence of potential misclassification of patients with an ADR report of lupus-like syndrome, we conducted one analysis where we only included ADR reports submitted by physicians and three analyses with different definitions of lupus-like syndrome (Table 2). First, cases of lupus-like syndrome were defined as only a report with the preferred term "LE Systemic" in the database. Second, cases were defined as all ADR reports of lupus-like syndrome and ADR reports that listed the presence of antinuclear antibodies (ANA) (preferred terms "Antinuclear factor test positive," "Antinuclear factor," and "Anti-DNA antibodies") and/or lupus erythematosus (LE) cells (preferred terms "LE test abnormal" and "LE cells present") (Table 2) 23. Third, lupus-like syndrome was defined as only a report with the preferred term "LE Systemic," which were verified by the presence of ANA and/or LE cells.

\section{RESULTS}

In VigiBase, we identified 3362 reports of lupus-like syndrome (cases) that were matched with 27092 reports of other ADRs (non-cases). Of the 3362 cases of lupus-like syndrome, 421 (12.4\%) cases listed an ADR of lupus-like syndrome with the presence of ANA and/or LE cells. The distribution of baseline characteristics for the cases of lupus-like syndrome and non-cases is shown in Table 3. The use of antiarrhythmic drugs, antihypertensive agents and non-steroidal anti-inflammatory drugs was more frequently reported in cases of lupus-like syndrome than in non-cases. In 144 of the 3362 cases of lupus-like syndrome (4.3\%), statins were reported as suspected $(n=106)$ or concomitant drug $(n=38)$.

Table 4 shows the characteristics of 106 cases of lupus-like syndrome with reported statins as suspected drug. The ADR reports were coded with the WHO-ART preferred terms "Systemic Lupus Erythematosus," "DLE," "LE type reaction," "SLE-like symptoms," and "LE rash" and "LE-like butterfly rash". Of the 106 reported cases with statins, 22 cases reported positive ANA results of whom no one reported positive anti-dsDNA or LE cells results. Most of these cases were reported in the early 1990s and originated from the United States of America. Lovastatin and simvastatin were more often reported as the suspected drug for the occurrence of lupus-like syndrome than other types of statins. The range of time to onset of lupus-like syndrome after starting statins was 1 day to 15.8 years (mean, 18.6 months; median, 7.1 months). Of the 106 reported cases, 14 listed recovery of lupus-like syndrome, while 17 listed no recovery of lupus-like syndrome. In 45 of the 106 cases statin withdrawal was 
Table 3 Baseline characteristics of the study population

\begin{tabular}{|c|c|c|c|}
\hline \multirow[t]{2}{*}{ Characteristics } & Cases & Non-cases & $p$ value \\
\hline & $(n=3362)$ & \multicolumn{2}{|l|}{$(n=27092)$} \\
\hline Mean Age (SD), y & $50.6 \pm 19.2$ & $50.7 \pm 19.5$ & $N A^{*}$ \\
\hline \multicolumn{4}{|l|}{ Sex, \% } \\
\hline Male & $975(29.0)$ & $7986(29.5)$ & $N A^{*}$ \\
\hline Female & $2387(71.0)$ & $19106(70.5)$ & $N A^{*}$ \\
\hline Reported ANA ${ }^{\dagger}, \%$ & $392(11.7)$ & - & - \\
\hline Reported Anti-dsDNA $\ddagger$ & $6(0.2)$ & - & - \\
\hline Reported LE cells§, \% & $23(0.7)$ & - & - \\
\hline \multicolumn{4}{|l|}{ Statins, \% } \\
\hline Suspected & $106(3.2)$ & $407(1.5)$ & $<0.001$ \\
\hline Concomitant & $38(1.1)$ & $318(1.2)$ & 0.768 \\
\hline \multicolumn{4}{|l|}{ Co-medication, \% } \\
\hline Anti-arrhythmic drugs, & $228(6.8)$ & $1140(4.2)$ & $<0.001$ \\
\hline Antihypertensives & $626(18.6)$ & $3293(12.2)$ & $<0.001$ \\
\hline Antidiabetics & $52(1.6)$ & $615(2.3)$ & 0.007 \\
\hline Non-statins Lipid modifying agents & $21(0.6)$ & $147(0.5)$ & 0.545 \\
\hline NSAIDs"l & $122(3.6)$ & $354(1.3)$ & $<0.001$ \\
\hline Corticosteroids & $115(3.4)$ & $376(1.4)$ & $<0.001$ \\
\hline DMARDs & $92(2.7)$ & $353(1.3)$ & $<0.001$ \\
\hline Antidepressants & $112(3.3)$ & $801(3.0)$ & 0.229 \\
\hline Antiepileptics & $88(2.6)$ & $420(1.6)$ & $<0.001$ \\
\hline Proton pump inhibitor agents & $68(2.0)$ & $386(1.4)$ & 0.007 \\
\hline Intestinal anti-inflammatory agents & $22(0.7)$ & $66(0.2)$ & $<0.001$ \\
\hline
\end{tabular}

reported, while in 6 cases no change in dose regimen was reported. Of these 45 cases where statins were withdrawn, 35 listed that lupus-like syndrome abated, while 6 listed no effect.

Of the specific 35 cases who reported that lupus-like syndrome abated on withdrawal of statins, 7 cases reported recovery, whereas 2 cases reported no recovery. Seven cases reported a rechallenge with statins of which four resulted in the recurrence of lupus-like syndrome. 
Table 4 Detailed information on the 106 case reports of lupus-like syndrome with statins as suspected drug.

\begin{tabular}{|c|c|c|c|}
\hline Characteristics & $\begin{array}{c}\text { No. } \\
\text { suspected } \\
\text { statins } \\
(n=106)\end{array}$ & Characteristics & $\begin{array}{c}\text { No. } \\
\text { suspected } \\
\text { statins } \\
(n=106)\end{array}$ \\
\hline Year of reporting & & Time to onset (days) & \\
\hline $1980-1990$ & 9 & $1-90$ & 22 \\
\hline 1990-1999 & 65 & $91-365$ & 20 \\
\hline 2000-2006 & 32 & $>365$ & 25 \\
\hline Type of statin & & Not recorded & 39 \\
\hline Simvastatin & 31 & Outcome & \\
\hline Pravastatin & 19 & Recovered & 14 \\
\hline Lovastatin & 33 & Not (or not yet) recovered & 17 \\
\hline Atorvastatin & 15 & Recovered with sequelae & 5 \\
\hline Fluvastatin & 4 & Died-reaction may be contributory & 1 \\
\hline Cerivastatin & 3 & Not recorded & 69 \\
\hline Rosuvastatin & 1 & Dechallenge (Action) & \\
\hline Country of origin & & Drug withdrawn & 45 \\
\hline Australia & 4 & Dose not changed & 6 \\
\hline Austria & 1 & Not recorded & 55 \\
\hline Belgium & 1 & Dechallenge (Result) & \\
\hline Canada & 3 & Reaction abated ${ }^{*}$ & 35 \\
\hline Finland & 2 & No effect observed & 6 \\
\hline France & 7 & Not applicable & 8 \\
\hline Germany & 9 & Not recorded & 57 \\
\hline Great Britain & 11 & Rechallenge (Action) & \\
\hline New Zealand & 1 & Rechallenge & 7 \\
\hline South Africa & 1 & No rechallenge & 16 \\
\hline Spain & 3 & Not recorded & 83 \\
\hline Sweden & 4 & Rechallenge (Result) & \\
\hline United States of America & 59 & Reaction recurred & 4 \\
\hline Reporter & & No recurrence & 1 \\
\hline General Practitioner & 24 & Not applicable & 16 \\
\hline Physician & 8 & Not recorded & 85 \\
\hline Specialist & 3 & Causality & \\
\hline Hospital & 7 & Probable & 7 \\
\hline Pharmacist & 1 & Possible & 14 \\
\hline Consumer & 1 & Not (yet) assessed & 3 \\
\hline \multirow[t]{2}{*}{ Other $^{\dagger}$} & 62 & Not assessed & 2 \\
\hline & & Not recorded & 80 \\
\hline
\end{tabular}

* Of the specific 35 ADR reports that reported abatement of lupus-like syndrome, 7 reported that the patient recovered after discontinuation of statins, while 2 reported no recovery upon withdrawal of statins. " "Other" includes consumer reports and various types of reports from other health professionals than physicians or dentists. 


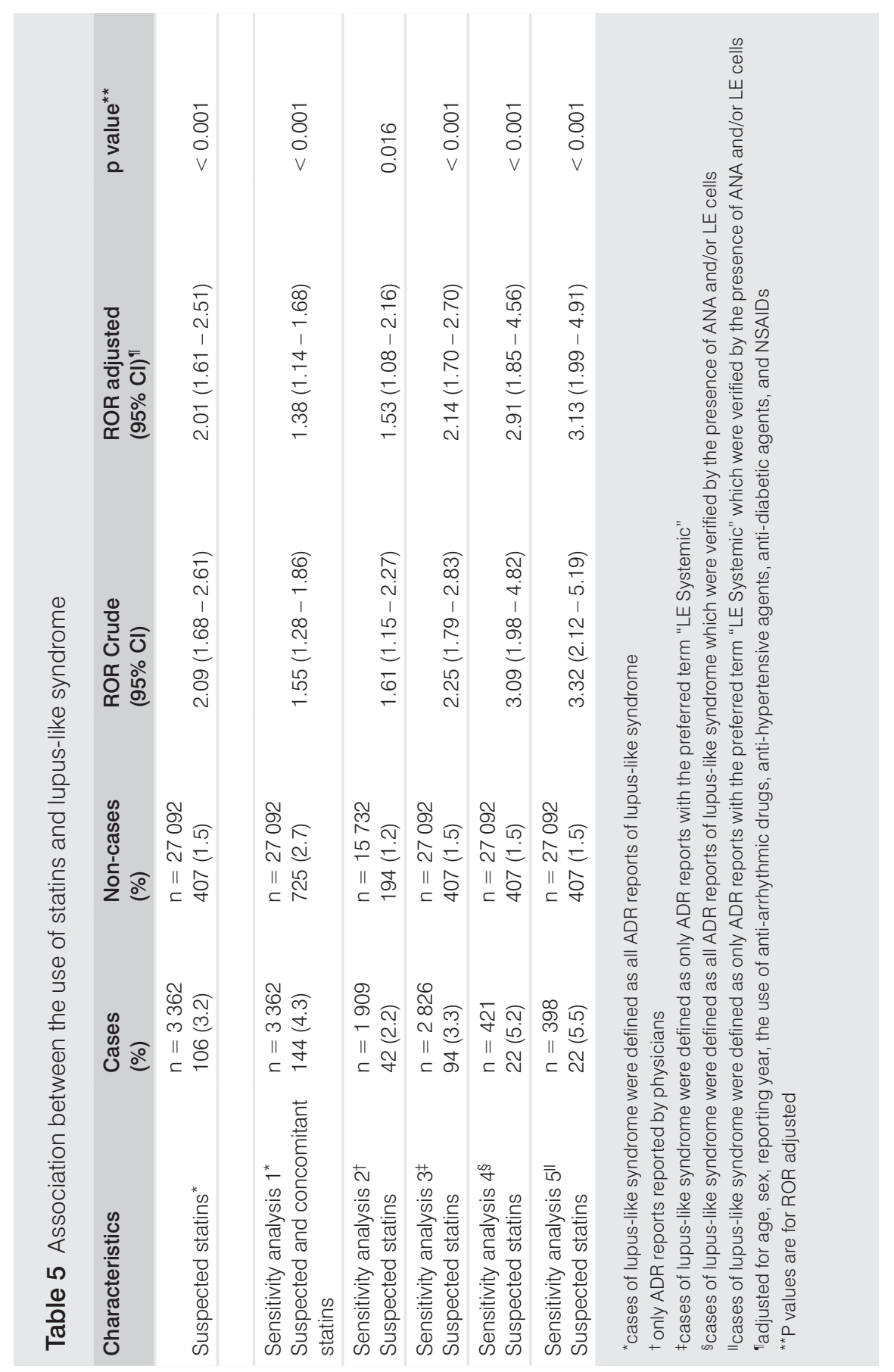


The association between the use of statins and lupus-like syndrome is shown in Table 5. Overall, statins were more often reported with lupus-like syndrome than other ADRs (adjusted ROR, 2.01 [95\% Cl 1.61 to 2.51]; $p=0.001$ ). The results were similar when we conducted the five sensitivity analyses. By changing the exposure to statins in suspected or concomitant drug for an ADR, the adjusted ROR for the occurrence of lupus-like syndrome was $1.38(95 \% \mathrm{Cl} 1.14$ to $1.68 ; \mathrm{p}=0.001)$. Including only ADR reports from physicians did not influence the results (adjusted ROR 1.53 [95\% Cl 1.08 to 2.16]; $p=0.016$ ). When we restricted our cases of lupus-like syndrome to only ADR reports with the preferred term "LE Systemic," the ROR for the association between statin use and the occurrence of lupus-like syndrome was 2.14 (95\% Cl 1.70 to 2.70; $p=0.001$ ). Similar results were obtained when we defined lupus-like syndrome as all ADR reports of lupus- like syndrome, which could be verified by the presence of ANA and/or LE cells (adjusted ROR 2.91 [95\% Cl 1.85 to 4.56]; $p=0.001$ ). Results remained similar when we included only ADR reports with the preferred term "LE Systemic" and ADR reports in which the presence of ANA and/or LE cells was listed (adjusted ROR 3.13 [95\% Cl 1.99 to 4.91]; $p=0.001$ ).

\section{DISCUSSION}

In the present study, we found a 2 -fold increased risk of reporting the use of statins in relation to the reporting of lupus-like syndrome compared with other ADR reports in VigiBase. Our findings were relatively insensitive to variations in the definition of lupuslike syndrome and the inclusion of reports that listed concomitant use of statins.

Several published case reports support the notion that statins could facilitate the development of lupus-like syndrome. Fourteen cases of lupus-like syndrome were reported in the literature, of whom four were considered drug-induced lupus, six lupus-like syndrome, one SLE, and three drug-induced subacute cutaneous lupus erythematosus (Table 1) 9-20. The cases in our study and the patients described in the published case reports were, on average, 50 years of age and predominantly female. Based on all the ADR reports in our study, lovastatin and simvastatin were more often reported as the suspected drug for the occurrence of lupus-like syndrome, whereas in the published case reports simvastatin was more often reported as the suspected drug. Based on the case reports in the medical literature, the course of lupus-like syndrome varies from case to case. Clinical improvement was seen in some patients within weeks after discontinuation of statins, while other cases showed clinical improvements only after treatment with immunosuppressive therapy. In our study, several ADR reports listed that the symptoms of lupus-like syndrome abated after discontinuation of statins. In these ADR reports, however, we could not ascertain whether the clinical improvements were related to statin withdrawal or to treatment 
with immunosuppressive therapy because the ADR reports did not present information on treatment regimes for lupus-like syndrome after discontinuation of statins. Suchak et al. reported a patient who developed subacute cutaneous lupus erythematosus on rechallenge with simvasatin ${ }^{18}$, hence suggesting a causal link. Of all the 106 ADR reports of lupus-like syndrome with statins as suspected drug, 4 reported lupus-like syndrome on rechallenge with statins.

In several case reports of lupus-like syndrome in the literature, the auto-antibodies remained positive months after withdrawal of statins despite clinical improvements. In our study, we had no data on laboratory evaluations (e.g. the presence of ANA) after the date of onset of lupus-like syndrome. Hence, the true underlying mechanism of statin-induced lupus-like syndrome is unclear, although in vitro and animal studies demonstrated that statins may exacerbate or trigger cellular apoptosis 27,28 and induce a shift in the T helper (Th) 1/Th2 balance leading to B-cell reactivity and production of pathogenic auto-antibodies 29,30. Recent studies have suggested that Treg cells can be unstable in the periphery and may promote autoimmunity 31,32 . According to these studies, we hypothesize that statins do not cause autoimmunity but may promote a pre-existing autoimmune-prone condition to progress toward clinical disease such as SLE 33.

There are several limitations in this study. First, due to underreporting of ADRs, only a fraction $(<10 \%)$ of the actual adverse events that occur are reported 34,35 . ADRs more likely to be reported than others are ADRs of relatively new drugs, severe ADRs, and ADRs that are not listed in the summary of product characteristics 34,36 . To control for possible time trends of reporting, we matched the non-cases for the calendar year of reporting.

Second, protopathic bias 37 could also have influenced the results. Since lupus-like syndrome and cardiovascular risk factors such as hyperlipidaemia often coexist 38,39 , and the diagnosis of lupus-like syndrome is often difficult to assess, lupus-like syndrome may already be present before the start of statin therapy. This may result in over-reporting of this ADR due to statin use. Data on the medical history of the patient are not available in the reports, making it impossible to control for protopathic bias. Third, the definition of lupus-like syndrome in our study was based on ADRs terms used in the ICSRs submitted by physicians, manufacturers, and consumers. In light of the heterogeneity of the ADR reports, we may have used a relatively sensitive, but non-specific, definition of lupus-like syndrome. The definition of lupus-like syndrome in our study could be a collection of several diseases such as SLE, drug-induced lupus, and acute, subacute and chronic cutaneous lupus erythematosus. The etiology and criteria for diagnosing these diseases are various 25,40,41. A patient is diagnosed with SLE when at least four of the eleven criteria from the guidelines from the American College of Rheumatology are present 42. Patients with drug-induced lupus, however, often do not meet the American College of Rheumatology criteria for 
SLE 43 . We did not have explicit information on the criteria set for making a distinction between these diseases, and for diagnosing lupus-like syndrome in our study population. To minimise misclassification of lupus-like syndrome, we conducted a sensitivity analysis where we defined lupus-like syndrome as a report with the WHO-ART preferred term "lupus erythematosus systemic" in which the presence of ANA and/or LE cells were listed. The association between statin use and the occurrence of lupus-like syndrome was still present. These markers are usually presented in $>80 \%$ of patients with SLE ${ }^{44}$. A limitation of this approach is that selection bias could have been introduced due to the absence of reporting ANA and/or LE cells in several ADR reports. WHO-ART adverse reaction terms do not provide preferred terms for negative ANA results, and therefore; we could not make a distinction between negative ANA results or underreporting of ANA. Another approach of minimizing misclassification of lupus-like syndrome was limiting the heterogeneity of the ADR reports by conducting a sensitivity analysis where we only included ADRs from physicians; the association between statin use and the occurrence of lupus-like syndrome was still present.

Fourth, we cannot exclude the possibility of unmeasured and/or inadequately measured residual confounding. In the present study, we did not account for the confounders such as smoking, family history, body mass index, sunlight and/or ethnicity 45,46.

Fifth, selective underreporting of the association between statin use and the occurrence of lupus-like syndrome may have occurred because the reporter may not always be alert on this possible association, especially when the patient was treated with more than one drug. As a result, the ADR of interest may not have been reported or the exposure to statins may have been defined as concomitant drug for an ADR. Therefore, we conducted a sensitivity analysis with all reports of statins classified as suspected or concomitant for an ADR, and we found an association between statin use and the occurrence of lupus-like syndrome, although weaker in effect.

To our knowledge, this is the first study to assess the association between statin use and lupus-like syndrome in a large spontaneous reporting database. Our findings were consistent in various sensitivity analyses with regard to the definition of lupus-like syndrome and the exposure to statins.

\section{CONCLUSIONS}

The result of this study underlines findings of individual case reports suggesting that the use of statins may be associated with the occurrence of lupus-like syndrome. Our finding warrants awareness about the possibility of developing lupus-like syndrome in patients treated with statins. Additional studies are needed to further confirm our finding, establish causality, and perform an analysis of the quantitative risk of lupuslike syndrome possibly brought on by statins. 


\section{Acknowledgments}

The authors wish to acknowledge the help of $\mathrm{R}$. Janssen in providing background data for the study and K. Star in commenting on an early draft of the article. In addition, we thank the National Centres for their contribution to the WHO-ADR database. The opinions and conclusions in this study are not necessarily those of the various National Centres or of the World Health Organisation. 


\section{REFERENCES}

1. Krukemyer JJ, Talbert RL. Lovastatin: a new cholesterol-lowering agent. Pharmacotherapy 1987; 7(6): 198-210.

2. Bellosta S, Paoletti R, Corsini A. Safety of statins: focus on clinical pharmacokinetics and drug interactions. Circulation 2004; 109(23 Suppl 1): III50-7.

3. Baigent C, Keech A, Kearney PM, et al. Efficacy and safety of cholesterol-lowering treatment: prospective meta-analysis of data from 90,056 participants in 14 randomised trials of statins. Lancet 2005; 366(9493): 1267-78.

4. Colhoun HM, Betteridge DJ, Durrington PN, et al. Primary prevention of cardiovascular disease with atorvastatin in type 2 diabetes in the Collaborative Atorvastatin Diabetes Study (CARDS): multicentre randomised placebo-controlled trial. Lancet 2004; 364(9435): 685-96.

5. Sever PS, Dahlof B, Poulter NR, et al. Prevention of coronary and stroke events with atorvastatin in hypertensive patients who have average or lower-than-average cholesterol concentrations, in the Anglo-Scandinavian Cardiac Outcomes Trial--Lipid Lowering Arm (ASCOT-LLA): a multicentre randomised controlled trial. Lancet 2003; 361(9364): 1149-58.

6. Ridker PM, Danielson E, Fonseca FA, et al. Reduction in C-reactive protein and LDL cholesterol and cardiovascular event rates after initiation of rosuvastatin: a prospective study of the JUPITER trial. Lancet 2009; 373(9670): 1175-82.

7. Arnaud C, Braunersreuther V, Mach F. Toward immunomodulatory and anti-inflammatory properties of statins. Trends Cardiovasc Med 2005; 15(6): 202-6.

8. Kwak BR, Mulhaupt F, Mach F. Atherosclerosis: anti-inflammatory and immunomodulatory activities of statins. Autoimmun Rev 2003; 2(6): 332-8.

9. Ahmad A, Fletcher MT, Roy TM. Simvastatin-induced lupus-like syndrome. Tenn Med 2000; 93(1): $21-2$.

10. Ahmad S. Lovastatin-induced lupus erythematosus. Arch Intern Med 1991; 151(8): 1667-8.

11. Bannwarth B, Miremont G, Papapietro PM. Lupuslike syndrome associated with simvastatin. Arch Intern Med 1992; 152(5): 1093.

12. Hanson J, Bossingham D. Lupus-like syndrome associated with simvastatin. Lancet 1998; 352(9133): 1070.

13. Jiménez-Alonso J, Jaimez L, Sabio J, Hidalgo C, Leon L. Atorvastatin-induced reversible positive antinuclear antibodies. Am J Med 2002; 112(4): 329-30.

14. Khosla R, Butman AN, Hammer DF. Simvastatin-induced lupus erythematosus. South Med J 1998; 91(9): 873-4.

15. Noël B, Panizzon R. Lupus-like syndrome associated with statin therapy. Dermatology 2004; 208(3): 276-7.

16. Rudski L, Rabinovitch MA, Danoff D. Systemic immune reactions to HMG-CoA reductase inhibitors. Report of 4 cases and review of the literature. Medicine 1998; 77(6): 378-83.

17. Sridhar MK, Abdulla A. Fatal lupus-like syndrome and ARDS induced by fluvastatin. Lancet 1998; 352(9122): 114.

18. SuchakR, Benson K, Swale V. Statin-induced Ro/SSa-positive subacute cutaneous lupus erythematosus. Clin Exp Dermatol 2007; 32(5): 589-91.

19. Graziadei IW, Obermoser GE, Sepp NT, Erhart KH, Vogel W. Drug-induced lupus-like syndrome associated with severe autoimmune hepatitis. Lupus 2003; 12(5): 409-12.

20. Srivastava M, Rencic A, Diglio G, et al. Drug-induced, Ro/SSA-positive cutaneous lupus erythematosus. Arch Dermatol 2003; 139(1): 45-9.

21. Noël B. Lupus erythematosus and other autoimmune diseases related to statin therapy: a systematic review. J Eur Acad Dermatol Venereol 2007; 21(1): 17-24.

22. Golomb BA, Evans MA. Statin adverse effects: a review of the literature and evidence for a mitochondrial mechanism. Am J Cardiovasc Drugs 2008; 8(6): 373-418.

23. Lindquist M. Vigibase, the WHO global ICSR database system: basic facts. Drug Inf J 2008; 42: 408-19.

24. World Health Organisation. Guidelines for ATC classification and DDD assigment. Oslo, Norway: World Health Organisation Collaborating Centre for Drug Statistics Methodology - Nordic Council on Medicines, 1999. 
25. Rubin RL. Drug-induced lupus. Toxicology 2005; 209(2): 135-47.

26. Greenland S, Robins JM. Confounding and misclassification. Am J Epidemiol 1985; 122(3): 495-506.

27. Blanco-Colio LM, Villa A, Ortego M, Hernández-Presa MA, Pascual A, Plaza JJ, Egido J. 3-Hydroxy-3-methyl-glutaryl coenzyme A reductase inhibitors, atorvastatin and simvastatin, induce apoptosis of vascular smooth muscle cells by downregulation of Bcl-2 expression and Rho A prenylation. Atherosclerosis 2002; 161(1): 17-26.

28. Chapman-Shimshoni D, Yuklea M, Radnay J, Shapiro H, Lishner M. Simvastatin induces apoptosis of B-CLL cells by activation of mitochondrial caspase 9. Exp Hematol 2003; 31(9): 779-83.

29. Youssef S, Stüve O, Patarroyo JC, et al. The HMG-CoA reductase inhibitor, atorvastatin, promotes a Th2 bias and reverses paralysis in central nervous system autoimmune disease. Nature 2002; 420(6911): 78-84.

30. Hakamada-Taguchi R, Uehara Y, Kuribayashi K, et al. Inhibition of hydroxymethylglutaryl-coenzyme a reductase reduces Th1 development and promotes Th2 development. Circ Res 2003; 93(10): 948-56.

31. Zhou X, Bailey-Bucktrout SL, Jeker LT, et al. Instability of the transcription factor Foxp3 leads to the generation of pathogenic memory T cells in vivo. Nat Immunol 2009; 10(9): 1000-7.

32. Komatsu N, Mariotti-Ferrandiz ME, Wang Y, Malissen B, Waldmann H, Hori S. Heterogeneity of natural Foxp3+ T cells: a committed regulatory T-cell lineage and an uncommitted minor population retaining plasticity. Proc Natl Acad Sci U S A 2009; 106(6): 1903-8.

33. Bonelli M, Smolen JS, Scheinecker C. Treg and lupus. Ann Rheum Dis 2010; 69 Suppl 1: i65-66.

34. Martin RM, Kapoor KV, Wilton LV, Mann RD. Underreporting of suspected adverse drug reactions to newly marketed ("black triangle") drugs in general practice: observational study. BMJ 1998; 317(7151): 119-20.

35. Lumley CE, Walker SR, Hall GC, Stanton N, Grob PR. The under-reporting of adverse drug reactions in general practice. Pharmaceut Med 1986; 1: 205-12

36. Milstien JB, Faich GA, Hsu JP, Knapp DE, Baum C, Dreis MW. Factors affecting physician reporting of adverse drug reactions. Drug Inf J 1986; 20: 157-64.

37. Horwitz RI, Feinstein AR. The problem of "protopathic bias" in case-control studies. Am J Med 1980; 68(2): $255-8$

38. Santos MJ, Vinagre F, Silva JJ, Gil V, Fonseca JE. Cardiovascular risk profile in systemic lupus erythematosus and rheumatoid arthritis: a comparative study of female patients. Acta Reumatol Port 2010; 35(3): 325-32.

39. Gabriel S. Heart disease in rheumatoid arthritis: changing the paradigm of systemic inflammatory disorders. J Rheumatol 2007; 34(1): 220-3

40. Vasoo S. Drug-induced lupus: an update. Lupus 2006; 15(11): 757-61.

41. Vedove CD, Del Giglio M, Schena D, Girolomoni G. Drug-induced lupus erythematosus. Arch Dermatol Res 2009; 301(1): 99-105.

42. Petri M. Review of classification criteria for systemic lupus erythematosus. Rheum Dis Clin North Am 2005; 31(2): 245-54, vi

43. Lee KJ, Wenzel MM. Systemic lupus erythematosus-like syndrome. In: Drug-induced diseases: prevention, detection, and management. Second ed. American Society of Health-system Pharmacists Inc. Bethesda, 2010. pp. 98-110.

44. Tomer Y, Buskila D, Shoenfeld Y. Pathogenic significance and diagnostic value of lupus autoantibodies. Int Arch Allergy Immunol 1993; 100(4): 293-306.

45. Bengtsson AA, Rylander L, Hagmar L, Nived O, Sturfelt G. Risk factors for developing systemic lupus erythematosus: a case-control study in southern Sweden. Rheumatology (Oxford) 2002; 41(5): 563-71.

46. Washio M, Horiuchi T, Kiyohara C, et al. Smoking, drinking, sleeping habits, and other lifestyle factors and the risk of systemic lupus erythematosus in Japanese females: findings from the KYSS study. Mod Rheumatol 2006; 16(3): 143-50. 


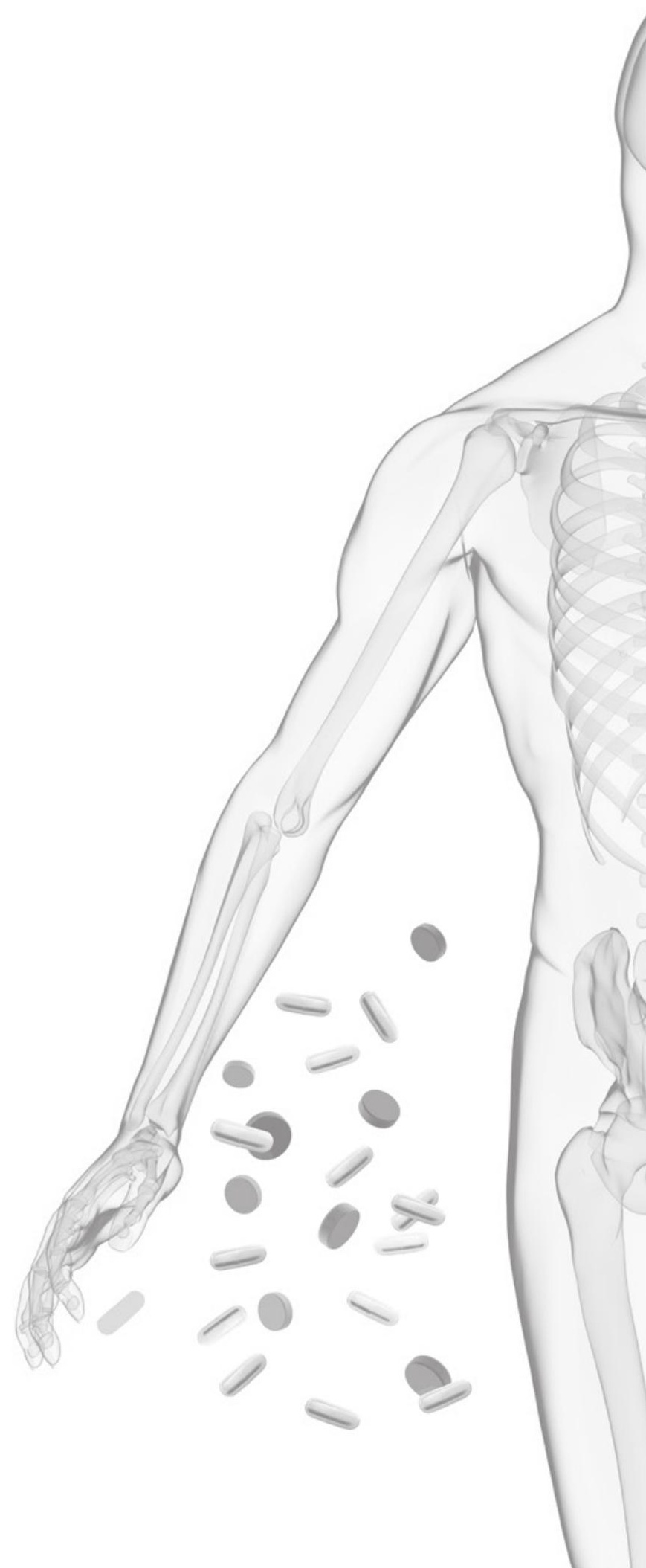




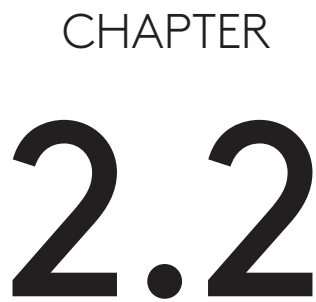

STATIN-ASSOCIATED POLYMYALGIA

RHEUMATICA: AN ANALYSIS USING WHO GLOBAL INDIVIDUAL CASE SAFETY DATABASE:

A CASE/NON-CASE APPROACH

H. J. I. de Jong, S. R. F. Saldi, O. H. Klungel, R. J. Vandebriel, P.C. Souverein,

R. H. B. Meyboom, J. L. M. Passier, H. van Loveren, JW. Cohen Tervaert 


\section{ABSTRACT}

\section{Objective}

To assess whether there is an association between statin use and the occurrence of polymyalgia rheumatic (PMR) in the spontaneous reporting database of the World Health Organisation (WHO).

\section{Methods}

We conducted a case/non-case study based on individual case safety reports (ICSR) in the WHO global ICSR database (VigiBase). Case reports containing the adverse event term polymyalgia rheumatica (WHOART or MedDRA Preferred Term) were defined as cases. Non-cases were all case reports containing other adverse event terms. Each case was matched to five non-cases by age, sex, and time of reporting. Case reports regarding a statin as suspected or concomitant drug were identified using the Anatomical Therapeutic Chemical (ATC) classification. Multivariate logistic regression was used to calculate reporting odds ratios (RORs) with 95\% confidence intervals $(\mathrm{Cl})$.

\section{Results}

We identified 327 reports of PMR as cases and 1635 reports of other ADRs as non-cases. Among cases, statins were more frequently reported as suspected agent (29.4\%) compared to non-cases (2.9\%). After adjustment for several covariates, statins were significantly associated with reports of PMR (ROR $14.21 ; 95 \% \mathrm{Cl} 9.89$ to 20.85).

\section{Conclusions}

The results of this study lends support to previous anecdotal case reports in the literature suggesting that the use of a statin may be associated with the occurrence of PMR. Further studies are needed to study the strength of the association in more detail and to elucidate the underlying mechanism. 


\section{INTRODUCTION}

The 3-hydroxy-3-methylglutaryl coenzyme A (HMG-CoA) reductase inhibitors, or statins, effectively lower cholesterol levels and significantly reduce the risk of cardiovascular events ${ }^{1}$. Recently, several studies have shown that these agents have anti-inflammatory and immunomodulatory properties which may eventually lead to immune dysregulation 2,3. Hence, statins might facilitate the development of autoimmunity, eventually resulting in autoimmune diseases. Previously, we observed in a population-based study that statins were associated with an increased risk of developing RA 4. Furthermore, cases of statin-associated lupus-like syndrome, dermatomyositis, and vasculitis have been reported ${ }^{5-11}$. Moreover, in a study comprising data from spontaneous case reports we have found an association between statin use and the occurrence of a lupus-like syndrome ${ }^{12}$. Three case reports suggested that statin use can trigger the development of polymyalgia rheumatica (PMR) ${ }^{9-11}$. PMR is an inflammatory rheumatic disease predominantly seen in the elderly and characterised by muscle pain and morning stiffness in the neck, shoulders, and/or pelvic girdle 13,14. The association between statin use and PMR has not yet been studied in depth. Therefore, we evaluated the association between statin use and the occurrence of PMR, using a case/non-case approach, in Vigibase the database of the WHO Uppsala Monitoring Centre containing individual case safety reports (ICSRs) of adverse drug reactions (ADRs).

\section{METHODS}

\section{Study Population}

The association between the use of statins and PMR was evaluated using the database of the World Health Organisation Uppsala Monitoring Centre (WHO UMC), Sweden. The database (VigiBase) contains the global ICSRs of suspected adverse reactions to pharmaceutical products submitted through National Pharmacovigilance Centres by 90 countries around the world. When we extracted the data for the present study, the database contained more than 4.6 million ICSRs of suspected ADRs 15. At the national level, ADRs are reported by health-care professionals, pharmaceutical companies, and in some countries by patients. Details about suspected ADRs such as age, sex, reporting date, country, nature of the ADR, suspected drugs, concomitantly used drugs, and interacting drugs are available in the VigiBase. ADRs are coded according to WHO Adverse Reaction Terminology (WHO-ART) or the Medical Dictionary for Regulatory Activities (MedDRA) 15. The reported drugs are encoded using the WHO Drug Dictionary Enhanced, which includes the WHO Anatomical Therapeutic Chemical (ATC) classification 16. Information in these reports is not homogenous, at 
least with regard to origin, completeness of documentation or the likelihood that the suspected drugs caused the adverse events 15 .

\section{Design}

A case/non-case approach was used to evaluate the association between the use of statins and PMR. In VigiBase, cases were identified as all ICSRs of ADRs containing the WHO-ART or MedDRA preferred term 'polymyalgia rheumatica' ${ }^{15}$. Reports were only included when data on sex and age were available. Since we were interested in incident cases of PMR, ADR reports with the preferred term 'polymyalgia rheumatica aggravated' were excluded from the study. Each case was matched to five non-cases by age, sex, and calendar year of reporting. Non-cases were reports concerning all other adverse reactions.

\section{Definition of Exposure}

Exposure to statins was defined as the reporting of statins as a suspected or concomitant drug for an ADR. The ATC codes for statins were C10AA (HMG-CoA reductase inhibitors), C10BA (HMG-CoA reductase inhibitors in combination with other lipid modifying agents), and C10BX (HMG-CoA reductase inhibitors and other combinations) 16 .

\section{Covariates}

Concomitant medication such as drugs with immunomodulatory activity, i.e., use of anti-arrhythmic drugs, anti-hypertensive and anti-diabetic agents, non-steroidal antiinflammatory drugs (NSAIDs), antidepressants, anti-epileptics, and proton pump inhibitors were considered as covariates 17.

\section{Statistical Analysis}

Characteristics of the cases and non-cases were analysed using Student's t test, Chi-square test or Fisher's exact test as appropriate. Means and standard deviations (SD) or percentages were obtained for continuous and categorical variables, respectively. The association between reporting of statins and PMR was assessed using logistic regression analysis and expressed as Reporting Odds Ratios (ROR) accompanied with 95\% confidence intervals $(\mathrm{Cl})$. The ROR provides a ratio of the odds of exposure in reports of cases and non-cases. The ROR was calculated by dividing the numerator (the number of cases with statins as the suspected drug divided by the number of cases with another suspected drug) by the denominator (the number of non-cases with statins as the suspected drug divided by the number of non-cases with another suspected drug). Covariates that acted as confounders were included in the model if each of them induced a change of crude $\beta$ estimates of the exposure-outcome association of at least $10 \% 18$. All tests were two-sided with a 
rejection of the null hypothesis at a p value of less than 0.05 . All the analyses were conducted using SPSS 16.0 statistical software (SPSS Inc., Chicago, Illinois, USA).

\section{Sensitivity Analysis}

We carried out five sensitivity analyses: 1) since reporters may not always be aware of statin-associated PMR we expanded the definition of statin use by including reports where statins were classified as concomitant (i.e. unsuspected) drug for an ADR. To explore the influence of potential misclassification of patients with an ADR report of PMR, we conducted four additional sensitivity analyses: 2) including only ADR reports reported by physicians, 3) including only ADR reports of patients older than 50 years $19,20,4)$ defining cases of PMR as an ADR with the single WHO-ART or MedDRA adverse reaction term PMR in which statins were reported as suspected drug, and 5) defining cases of PMR as only ADR of WHO-ART adverse reaction term PMR in which statins were reported as suspected or concomitant drug. In the latter two analyses, ADRs with two or more WHO-ART adverse reaction terms were excluded from the analysis.

\section{RESULTS}

\section{Baseline Characteristics}

In VigiBase, we identified 327 reports of PMR (cases) that were matched with 1635 reports of other ADRs (non-cases). The distribution of baseline characteristics for cases of PMR and non-cases are shown in Table 1. Characteristics were similar between cases and non-cases except for anti-depressant and anti-arrhythmic drugs that were more frequently reported in non-cases than in patients with PMR. In 104 of 327 cases of PMR, statins were reported as suspected or concomitant drug. Of these 104 cases, 96 reported statins as suspected drug whereas 8 reported statins as concomitant drug. The distribution of statins reported as suspected drug in cases and non-cases are presented in figure 1. The characteristics of 96 cases of PMR with reported statins as suspected drug are presented in table 2. Most of these cases were reported by physicians in the late ' 90 s and mainly originated from the United States of America, Germany, and Great Britain. Simvastatin and atorvastatin were more often reported as the suspected drug than other types of statins. The time to onset of PMR after starting statins ranged from 1 day to 5.7 years (mean, 11.9 months; median, 3.7 months). In 26 of 96 ADR reports statin withdrawal was recorded. Of these 26 reported cases, 8 cases reported that PMR abated while 8 cases reported no effect. In six cases, a rechallenge with statins was reported resulting in the recurrence of PMR. 
Table 1 Baseline characteristics of the polymyalgia rheumatica study population

\begin{tabular}{lccc}
\hline Characteristics & $\begin{array}{c}\text { Cases } \\
(\mathbf{n}=327)\end{array}$ & $\begin{array}{c}\text { Non-cases } \\
(\mathbf{n = 1 6 3 5 )}\end{array}$ & p value \\
\hline Mean Age (SD), y & $67.7(11.0)$ & $67.7(11.0)$ & $N^{*}$ \\
\hline Age categories, \% & $20(6.1)$ & $100(6.1)$ & $N^{*}$ \\
\hline$<50$ & $307(93.9)$ & $1535(93.9)$ & $N^{*}$ \\
\hline$\geq 50$ & & & \\
\hline Sex, \% & $124(37.9)$ & $620(37.9)$ & $N^{*} A^{*}$ \\
\hline Male & $203(62.1)$ & $1015(62.1)$ & $N^{*}$ \\
\hline Female & & & \\
\hline Statins, \% & $96(29.4)$ & $47(2.9)$ & $<0.001$ \\
\hline Suspected & $104(31.8)$ & $129(7.9)$ & 0.08 \\
\hline Suspected or Concomitant & & & \\
\hline Co-medication, \% & $9(2.8)$ & $93(5.7)$ & 0.03 \\
\hline Anti-arrhythmic drugs & $85(26.0)$ & $354(21.7)$ & 0.09 \\
\hline Anti-hypertensive agents & $14(4.3)$ & $86(5.3)$ & 0.46 \\
\hline Anti-diabetic agents & $3(0.9)$ & $12(0.7)$ & 0.73 \\
\hline Non-statins Lipid modifying agents & $7(2.1)$ & $24(1.5)$ & 0.34 \\
\hline NSAIDs ${ }^{\dagger}$ & $8(2.5)$ & $25(1.5)$ & 0.24 \\
\hline Corticosteroids & $4(1.2)$ & $30(1.8)$ & 0.64 \\
\hline DMARDs ${ }^{\ddagger}$ & $4(1.2)$ & $74(4.5)$ & 0.003 \\
\hline Antidepressants & $1(0.3)$ & $25(1.5)$ & 0.10 \\
\hline Anti-epileptics & $15(4.6)$ & $59(3.6)$ & 0.40 \\
\hline Acid inhibitors & & & \\
\hline
\end{tabular}

*NA indicate not applicable because cases and non-cases were matched by age and sex †NSAIDs: Non-steroidal anti-inflammatory drugs

FDMARDs: Disease-modifying anti-rheumatic drugs

\section{Association between Reporting of Statins and PMR}

The association between the use of statins and PMR is shown in Table 3. Overall, statins were more often reported in patients with PMR in comparison with patients who had experienced other ADRs (adjusted ROR 14.21 [95\% Cl 9.89 to 20.85]; $p=0.001$ ).

The results were consistent when we conducted five sensitivity analyses; 1) by expanding the exposure definition to statins as suspected or concomitant drug for an ADR, the adjusted ROR for the occurrence of PMR was 6.03 (95\% Cl 4.40 to 8.25; 
A

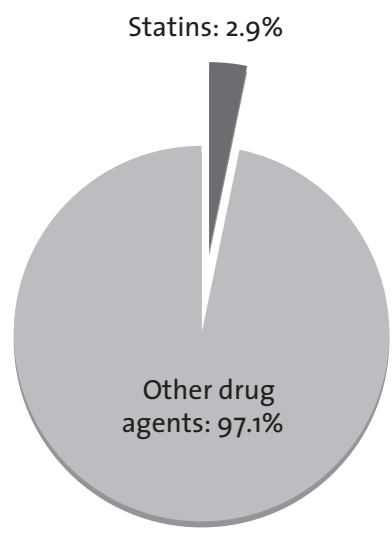

B

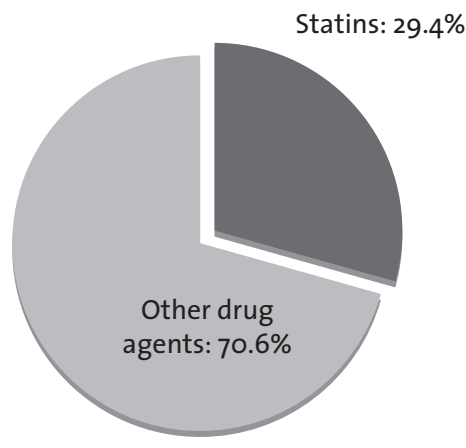

Figure 1 Exposure to statins in cases and non-cases

$1 \mathrm{~A}$ Exposure to drugs in reports of other adverse drug reactions (non-cases)

1B Exposure to drugs in reports of polymyalgia rheumatica (cases)

$p=0.001$ ), 2) including only ADR reports reported by physicians: (adjusted ROR 14.70 [95\% Cl 7.07 to 30.65]; $p=0.001$ ), 3) including only ADR reports of patients older than 50 years: (adjusted ROR 14.01 [95\% Cl 6.98 to 24.83]; $p=0.001$ ), 4) including only ADR reports of PMR without other ADRs, in which statins were reported as suspected drug: (adjusted ROR 13.90 [95\% Cl 8.65 to 22.35]; $p=0.001$ ), or 5) including only ADR reports of PMR without other ADRs and in addition where statins were reported as suspected or concomitant drug: (adjusted ROR 5.75 [95\% Cl 3.74 to 8.83]; $p=0.001$ ). 
Table 2 Detailed information on the 96 case reports with statins as suspected drug.

\begin{tabular}{|c|c|c|c|}
\hline Characteristics & $\begin{array}{c}\text { No. } \\
\text { suspected } \\
\text { statins } \\
(\mathrm{n}=96)\end{array}$ & Characteristics & $\begin{array}{c}\text { No. } \\
\text { suspected } \\
\text { statins } \\
(\mathrm{n}=96)\end{array}$ \\
\hline Year of reporting & & Time to onset (days) & \\
\hline 1990-1999 & 59 & $1-90$ & 35 \\
\hline 2000-2006 & 37 & $91-365$ & 17 \\
\hline Type of statin & & $>365$ & 23 \\
\hline Simvastatin & 35 & Not recorded & 21 \\
\hline Pravastatin & 8 & Outcome & \\
\hline Lovastatin & 10 & Recovered & 15 \\
\hline Atorvastatin & 26 & Not (or not yet) recovered & 23 \\
\hline Fluvastatin & 6 & Recovered with sequelae & 3 \\
\hline Cerivastatin & 6 & Not recorded & 55 \\
\hline Rosuvastatin & 5 & Dechallenge (Action) & \\
\hline Country of origin & & Drug withdrawn & 26 \\
\hline Australia & 9 & Dose not changed & 12 \\
\hline Canada & 4 & Not recorded & 58 \\
\hline Finland & 2 & Dechallenge (Result) & \\
\hline France & 2 & Reaction abated & 8 \\
\hline Germany & 18 & No effect observed & 8 \\
\hline Great Britain & 15 & Not applicable & 12 \\
\hline Ireland & 1 & Not recorded & 68 \\
\hline New Zealand & 3 & Rechallenge (Action) & \\
\hline Norway & 1 & Rechallenge & 6 \\
\hline Sweden & 3 & No rechallenge & 20 \\
\hline Switzerland & 1 & Not recorded & 70 \\
\hline The Netherlands & 3 & Rechallenge (Result) & \\
\hline United States of America & 34 & Reaction recurred & 6 \\
\hline Reporter & & Not applicable & 20 \\
\hline General Practitioner & 31 & Not recorded & 70 \\
\hline Physician & 8 & Causality & \\
\hline Specialist & 5 & Probable & 3 \\
\hline Hospital & 2 & Possible & 21 \\
\hline Manufacturer & 2 & Not assessed & 10 \\
\hline Consumer & 2 & Not recorded & 62 \\
\hline Other* & 2 & & \\
\hline Not reported & 44 & & \\
\hline
\end{tabular}




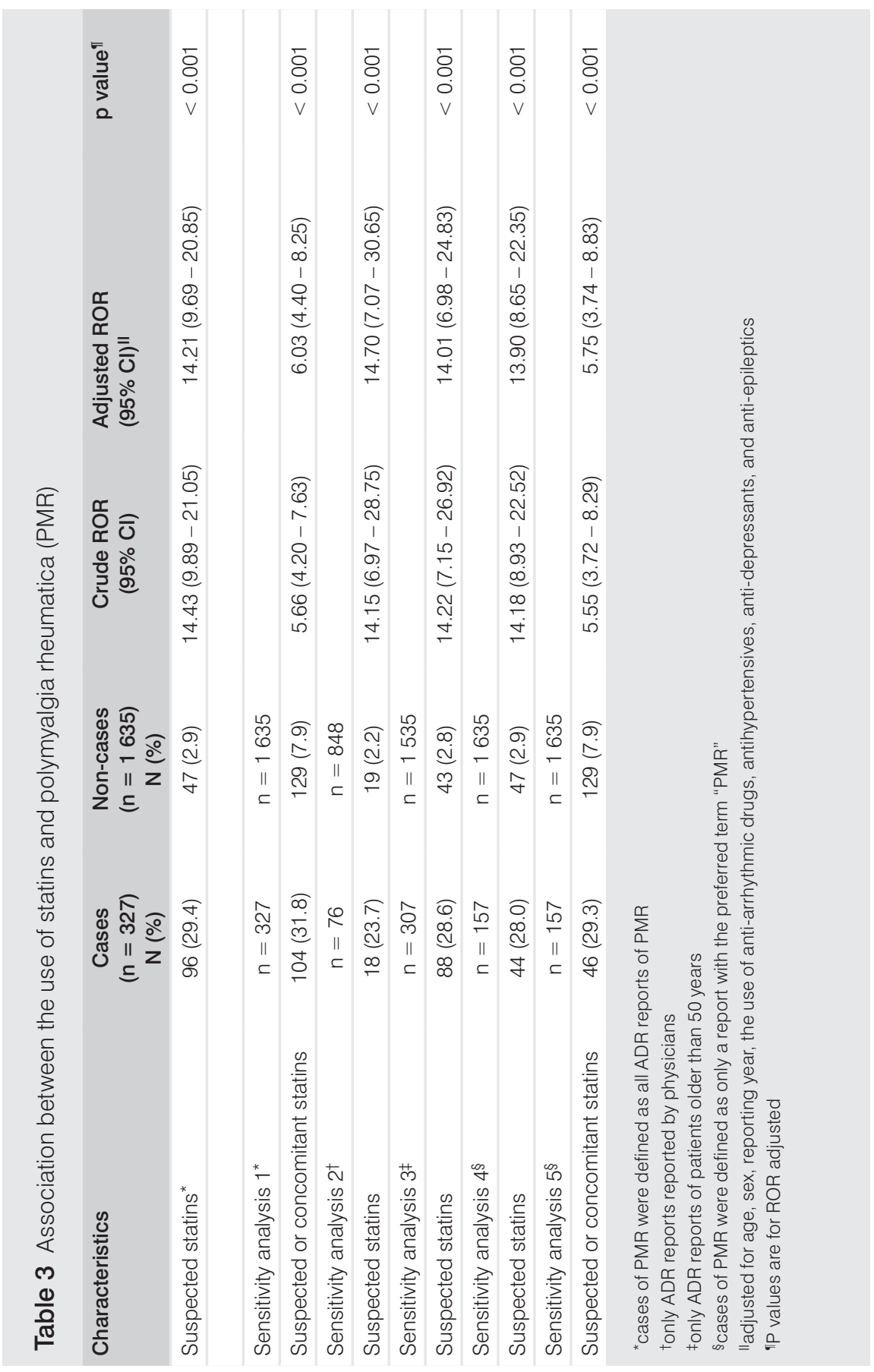




\section{DISCUSSION}

In the present study, we observed an association between statin use and reporting of PMR in VigiBase. In six reports the recurrence had been recorded of PMR after re-exposure to statins, which strengthened the suspicion regarding the drug's causal involvement in these patients. In the absence of information on the treatment of the PMR, in our study it could not be determined whether clinical improvement was related to statin discontinuation or to treatment with corticosteroids. In two previously published cases ${ }^{9-11}$, however, information on treatment regimes has been provided. The observation that clinical improvement had occurred within a month after discontinuation of the statin and without the administration of corticosteroids, was highly suggestive of a causal role of the statin in these two patients.

In our study, cases were predominantly older than 50 years and female (67\%) which is in line with published studies reporting incidence rates of non-drug associated PMR 21,22

Several limitations of our study need to be addressed. First, data were obtained from a spontaneous reporting system without additional clinical assessment or qualitative verifications by the authors. For instance, in many ADR reports no information was available about the withdrawal of statins, the course of PMR and the response to steroid therapy.

Second, there is gross but variable underreporting and it is likely that only a fraction of the actual adverse events that occurred (perhaps less than 10\%) has been reported 23,24. ADRs to relatively new drugs, severe ADRs, and ADRs which are not listed in the summary of product characteristics tend to be more often reported 23 . To control for possible time trends of reporting, we matched non-cases for the calendar year of reporting.

Third, we cannot exclude the possibility of unmeasured and/or inadequately measured residual confounding. Furthermore, in VigiBase confounders are difficult to determine because they were not always recorded in the reports. Unfortunately, with our patients no data were available on vitamin $D$ deficiency, which could be an important risk factor for statin-associated muscle complaints ${ }^{25}$. Recently, one case report 26 , case series 27 , and two cross sectional studies have found an association between vitamin D insufficiency and statin-induced myalgia 28,29. Importantly, vitamin $\mathrm{D}$ has been shown to modulate the immune response 30 . For instance in humans, high doses vitamin D therapy results in the inhibition of Thelper (Th) 1 and Th17 cells and the promotion of Th2 and regulatory T cells 31 .

Fourth, reporters may not be aware of the possible association between PMR and the use of statins use, and therefore it may not have been reported, or the statin may have been regarded as a concomitant drug only. In a sensitivity analysis including all reports of statins, suspected or not, the association between statin use and PMR was still observed, although somewhat attenuated. 
Fifth, clinical details about the patients with PMR were scarce and we were not able to recognise possible diagnostic misclassification ${ }^{32}$. The reports in Vigibase of PMR were submitted by medical specialists, GPs, manufactures or patients. Information on clinical features, such as elevation of the inflammatory markers (erythrocyte sedimentation rate (ESR) and C-reactive protein (CRP)), response to steroids and disease course, were often not recorded. However, when we only included case reports from physicians, the association between statin use and the occurrence of PMR was still present.

Since PMR occurs almost exclusively in patients aged 50 years and older 19,20, we performed a sensitivity analysis in which we excluded patients who were younger than 50 years from the study. We still found an association between statin use and the occurrence of PMR.

The symptoms of PMR are very characteristic, although other conditions may mimic PMR 13,14. Statins are an established cause of muscular injury and specifically the inclusion of cases of myositis, non-specific myalgias, and/or myopathy may have occurred, the more so since laboratory findings, i.e., serum CK were often not recorded. On the other hand, patients with statin-associated myopathy may have normal serum CK levels which make it sometimes difficult to distinguish PMR from myopathic syndromes ${ }^{33}$.

As yet, the possible pathophysiology underlying statin-associated PMR is uncertain. Recently, it has been postulated that in statin-associated necrotising myopathy, statins may induce neo-antigens as a result of muscle damage which are subsequently presented to the immune system ${ }^{34,35}$. A similar mechanism may be operative in statin-associated PMR.

We believe that the use of a case/non-case approach in a study with ICSRs of ADRs is, notwithstanding the limitations of our data, an appropriate approach in pharmacovigilance and drug safety research 36 . To our knowledge, this is the first study to assess the association between statin use and the occurrence of PMR in a large spontaneous reporting database. Our findings are consistent in various sensitivity analyses.

We postulate that the use of statins may be associated with an increased occurrence of PMR. Our study presents a pharmacovigilance signal and supports previous anecdotal case reports. We think that further research towards confirming and explaining the association between statin use and PMR is warranted.

\section{Acknowledgments}

The authors wish to acknowledge the help of Dr R. Janssen in providing background data for the study and K. Star in commenting on an early version of the manuscript. In addition, we thank the National Centres for their contribution to the WHO-ADR database. The opinions and conclusions in this study are not necessarily those of the various National Centres or of the World Health Organisation. 


\section{REFERENCES}

1. Baigent C, Keech A, Kearney PM, et al. Efficacy and safety of cholesterol-lowering treatment: prospective meta-analysis of data from 90,056 participants in 14 randomised trials of statins. Lancet 2005; 366(9493): 1267-78.

2. Arnaud $\mathrm{C}$, Braunersreuther $\mathrm{V}$, Mach F. Toward immunomodulatory and anti-inflammatory properties of statins. Trends Cardiovasc Med 2005; 15(6): 202-6.

3. Ridker PM, Danielson E, Fonseca FA, et al. Reduction in C-reactive protein and LDL cholesterol and cardiovascular event rates after initiation of rosuvastatin: a prospective study of the JUPITER trial. Lancet 2009; 373(9670): 1175-82.

4. De Jong HJ, Klungel OH, Van Dijk L, et al. Use of statins is associated with an increased risk of rheumatoid arthritis. Ann Rheum Dis 2012; 71(5): 648-54.

5. Noël B. Lupus erythematosus and other autoimmune diseases related to statin therapy: a systematic review. J Eur Acad Dermatol Venereol 2007; 21(1): 17-24.

6. Golomb BA, Evans MA. Statin adverse effects: a review of the literature and evidence for a mitochondrial mechanism. Am J Cardiovasc Drugs 2008; 8(6): 373-418.

7. Haroon M, Devlin J. A case of ANCA-associated systemic vasculitis induced by atorvastatin. Clin Rheumatol 2008; 27 Suppl 2: S75-7.

8. Sen D, Rosenstein E, Kramer N. ANCA-positive vasculitis associated with simvastatin/ezetimibe: expanding the spectrum of statin-induced autoimmunity? Int J Rheum Dis 2010; 13(3): e29-31.

9. Goëb V, Guillemant N, Vittecoq O, Le Loët X. Cerivastatin-induced polymyalgia rheumatica-like illness. Clin Rheumatol 2004; 23(2): 179.

10. Kay J, Finn D, Stone J. Case records of the Massachusetts General Hospital. Case 4-2006. A 79-year-old woman with myalgias, fatigue, and shortness of breath. N Engl J Med 2006; 354(6): 623-30.

11. Rudski L, Rabinovitch MA, Danoff D. Systemic immune reactions to HMG-CoA reductase inhibitors. Report of 4 cases and review of the literature. Medicine 1998; 77(6): 378-83.

12. De Jong HJ, Tervaert JW, Saldi SR, et al. Association between statin use and lupus-like syndrome using spontaneous reports. Semin Arthritis Rheum 2011; 41(3): 373-81.

13. Samanta A, Kendall J. A fresh look at polymyalgia rheumatica. Rheumatology (Oxford) 2002; 41 (12): 1455-6.

14. Salvarani C, Cantini F, Boiardi L, Hunder GG. Polymyalgia rheumatica and giant-cell arteritis. N Engl J Med 2002; 347(4): 261-71.

15. Lindquist M. Vigibase, the WHO global ICSR database system: basic facts. Drug Inf J 2008; 42: 408-19.

16. World Health Organisation. Guidelines for ATC classification and DDD assigment. Oslo, Norway: World Health Organisation Collaborating Centre for Drug Statistics Methodology - Nordic Council on Medicines, 1999.

17. Dedeoglu F. Drug-induced autoimmunity. Curr Opin Rheumatol 2009; 21(5): 547-51.

18. Greenland S, Robins JM. Confounding and misclassification. Am J Epidemiol 1985; 122(3): 495-506.

19. Chuang TY, Hunder GG, IIstrup DM, Kurland LT. Polymyalgia rheumatica: a 10-year epidemiologic and clinical study. Ann Intern Med 1982; 97(5): 672-80.

20. Healey LA. Long-term follow-up of polymyalgia rheumatica: Evidence for synovitis. Semin Arthritis Rheum 1984; 13: 322-8.

21. Smeeth L, Cook C, Hall AJ. Incidence of diagnosed polymyalgia rheumatica and temporal arteritis in the United Kingdom, 1990-2001. Ann Rheum Dis 2006; 65(8): 1093-8.

22. Salvarani C, Gabriel SE, O'Fallon WM, Hunder GG. Epidemiology of polymyalgia rheumatica in Olmsted County, Minnesota, 1970-1991. Arthritis Rheum 1995; 38(3): 369-73.

23. Lumley CE, Walker SR, Hall GC, Stanton N, Grob PR. The under-reporting of adverse drug reactions in general practice. Pharmaceut Med 1986; 1: 205-12.

24. Martin RM, Kapoor KV, Wilton LV, Mann RD. Underreporting of suspected adverse drug reactions to newly marketed ("black triangle") drugs in general practice: observational study. BMJ 1998; 317(7151): 119-20.

25. Gupta A, Thompson PD. The relationship of vitamin D deficiency to statin myopathy. Atherosclerosis 2011; 215(1): 23-9. 
26. Bell DS. Resolution of statin-induced myalgias by correcting vitamin D deficiency. South Med J 2010; 103(7): 690-2

27. Lee P, Greenfield JR, Campbell LV. Vitamin D insufficiency--a novel mechanism of statin-induced myalgia? Clin Endocrinol (Oxf) 2009; 71(1): 154-5

28. Ahmed W, Khan N, Glueck CJ, et al. Low serum $25(\mathrm{OH})$ vitamin D levels $(<32 \mathrm{ng} / \mathrm{mL})$ are associated with reversible myositis-myalgia in statin-treated patients. Transl Res 2009; 153(1): 11-6.

29. Duell PB, Connor WE. Abstract 3701: Vitamin D deficiency is associated with myalgias in hyperlipidemic subjects taking statins. Circulation 2008; 118: S470.

30. Peelen E, Knippenberg S, Muris $\mathrm{AH}$, et al. Effects of vitamin D on the peripheral adaptive immune system: A review. Autoimmun Rev 2011; 10(12): 733-43.

31. Smolders J, Peelen E, Thewissen M, Cohen Tervaert JW, Menheere P, Hupperts R, Damoiseaux J. Safety and $T$ cell modulating effects of high dose vitamin D3 supplementation in multiple sclerosis. PLoS One 2010; 5(12): e15235.

32. Dasgupta B, Borg FA, Hassan N, et al. BSR and BHPR guidelines for the management of polymyalgia rheumatica. Rheumatology (Oxford) 2010; 49(1): 186-90.

33. Phillips $\mathrm{P}$, Haas R, Bannykh S, et al. Statin-associated myopathy with normal creatine kinase levels. Ann Intern Med 2002; 137(7): 581-5.

34. Grable-Esposito P, Katzberg HD, Greenberg SA, Srinivasan J, Katz J, Amato AA. Immune-mediated necrotizing myopathy associated with statins. Muscle Nerve 2010; 41(2): 185-90.

35. Christopher-Stine L, Casciola-Rosen LA, Hong G, Chung T, Corse AM, Mammen AL. A novel autoantibody recognizing $200-\mathrm{kd}$ and $100-\mathrm{kd}$ proteins is associated with an immune-mediated necrotizing myopathy. Arthritis Rheum 2010; 62(9): 2757-66.

36. Wilson AM, Thabane L, Holbrook A. Application of data mining techniques in pharmacovigilance. $\mathrm{Br} \mathrm{J}$ Clin Pharmacol 2004; 57(2): 127-34 


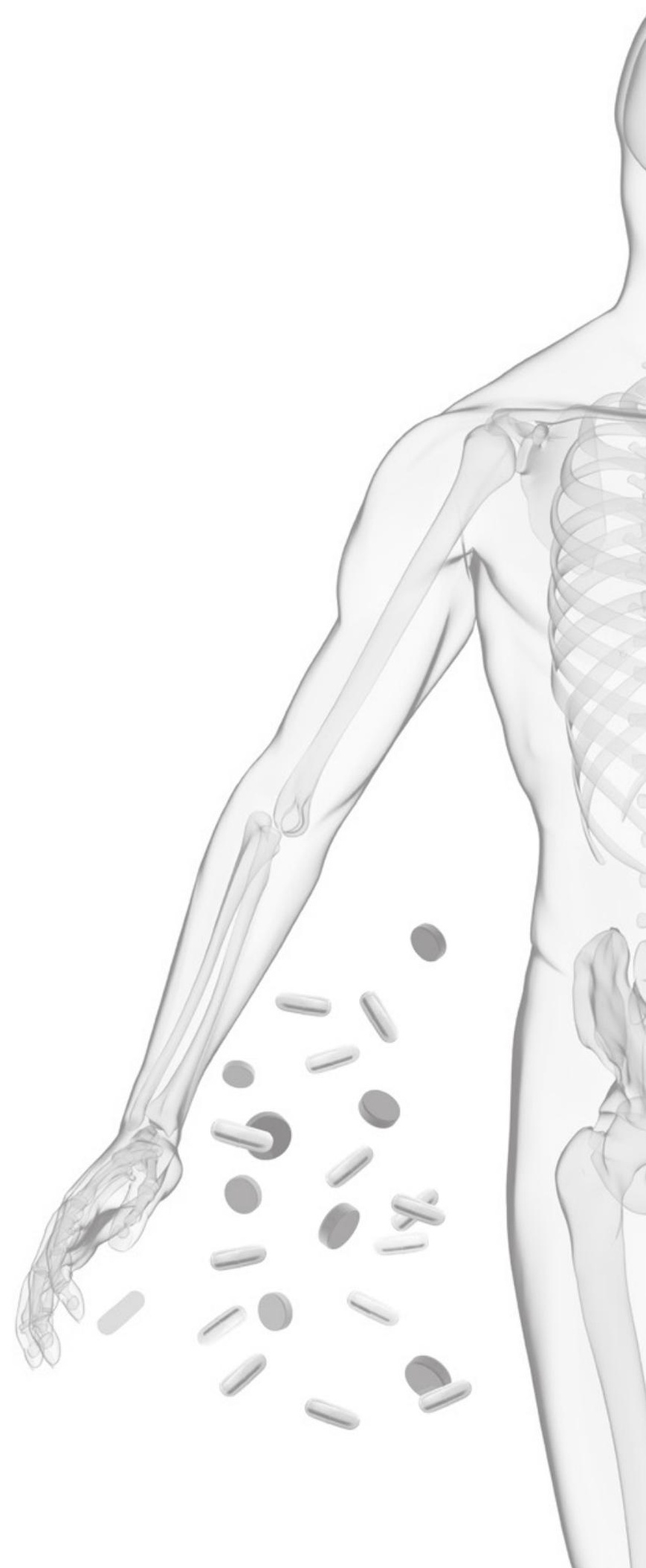




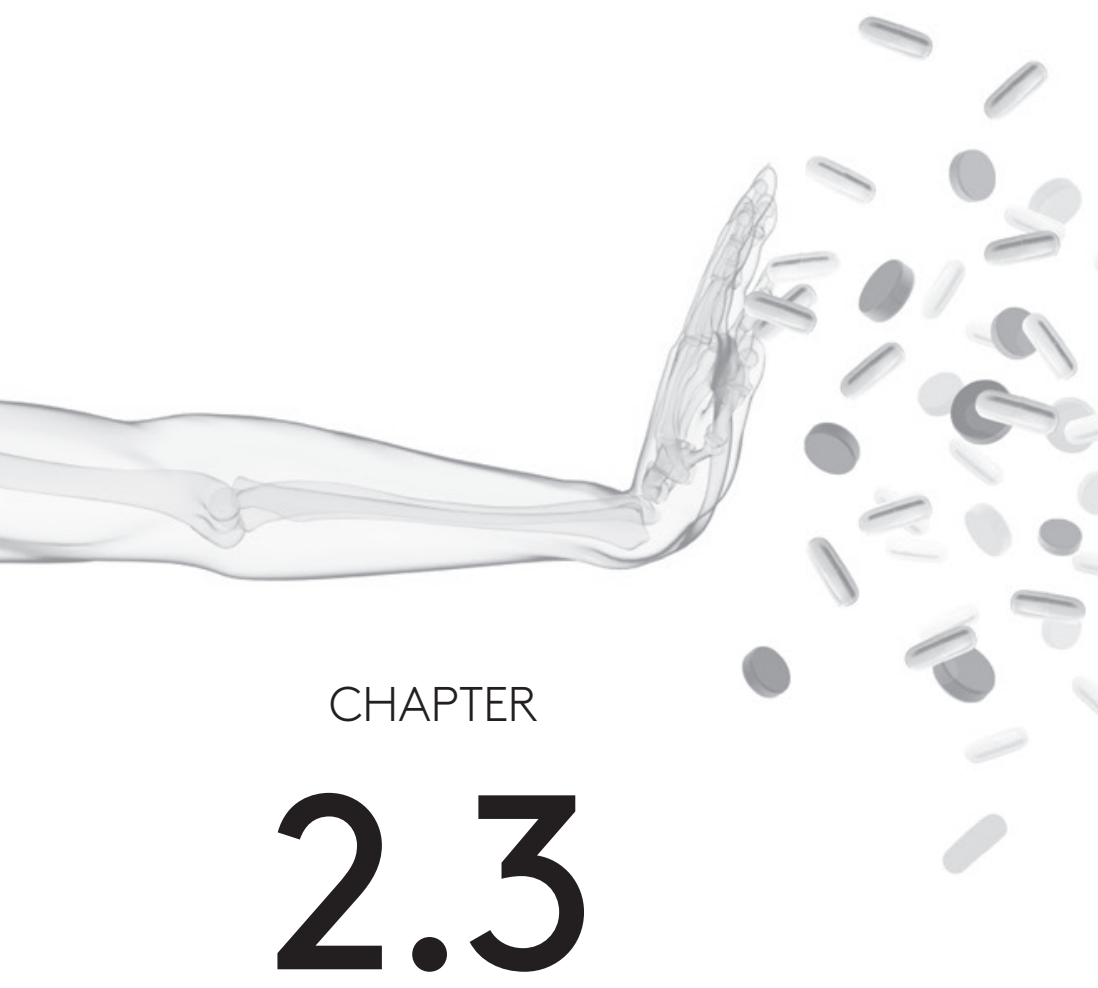

\section{GIANT CELL ARTERITIS AND POLYMYALGIA RHEUMATICA AFTER RE-EXPOSURE TO A STATIN: A CASE REPORT}

H.J.I. De Jong, R.H.B. Meyboom, M.J. Helle, O.H. Klungel, L. Niskanen, JW. Cohen Tervaert

Ann Intern Med 2014; 161(8): 614-615 



\section{CASE REPORT}

\section{Background}

Statins, or 3-hydroxy-3-methylglutaryl coenzyme A reductase inhibitors, are widely used to prevent cardiovascular diseases and are generally regarded as safe. However, adverse reactions, such as myalgia, myositis, rhabdomyolysis, muscle weakness and cramps with or without elevated serum creatine kinase levels may occur ${ }^{1}$. Furthermore, we have recently described that statins may also be associated with polymyalgia rheumatica (PMR) 2 .

\section{Case report}

A 78-year-old woman presented in December 2003 with muscle pain in the hips and shoulders and morning stiffness. She had a history of hypercholesterolaemia treated with atorvastatin $10 \mathrm{mg} /$ day for nine months and hypertension treated with candesartan $8 \mathrm{mg} /$ day and bisoprolol $2.5 \mathrm{mg} /$ day. Laboratory studies revealed an erythrocyte sedimentation rate (ESR) of $61 \mathrm{~mm} / \mathrm{h}$ and platelet count of $487 \times 10^{9} \mathrm{cells} / \mathrm{L}$. She was diagnosed with PMR and advised to stop atorvastatin therapy. Her symptoms gradually resolved. Five months after presentation, ESR was normal at $13 \mathrm{~mm} / \mathrm{h}$.

In February 2006, her low-density lipoprotein cholesterol level was $4.5 \mathrm{mmol} / \mathrm{L}$ (174 $\mathrm{mg} / \mathrm{dL}$ ), and therapy with rosuvastatin $5 \mathrm{mg}$ twice weekly was resumed. Symptoms of PMR recurred and progressed, and she reported visual disturbances.

Temporal arteries were tender on palpation. Hematoxylin-eosin staining of a biopsy specimen of the temporal artery showed panarteritis comprising lymphocytes, neutrophils, eosinophils and macrophages with giant cells (Figure 1). Intimal thickening with clear luminal narrowing was also noted (Figure 2). An ESR of $95 \mathrm{~mm} / \mathrm{h}$ and a C-reactive protein (CRP) level of $2381.0 \mathrm{nmol} / \mathrm{L}(250 \mathrm{mg} / \mathrm{L}$ ) were found on laboratory studies. Giant cell arteritis (GCA) with PMR was diagnosed.

Rosuvastatin therapy was discontinued, and the patient was treated with prednisolone $40 \mathrm{mg} /$ day, the dosage of which was slowly tapered. Her symptoms abated over the next few months, and the ESR and CRP level decreased to $6 \mathrm{~mm} / \mathrm{h}$ and less than $95.2 \mathrm{nmol} / \mathrm{L}$ (10 mg/L), respectively.

In June 2008, prednisolone therapy was stopped. A few months later, PMR and GCA relapsed. This time, relapse was not preceded by statin therapy. Again, the patient received corticosteroids, which resulted in a good response until she died in February 2009.

\section{Discussion}

The patient's presentation strongly suggested statin-induced PMR and GCA. Both are part of the same disease spectrum and are associated with the HLA-DRB1*04 allele $^{3}$, the presence of which was not determined in this patient. Between 16 to $21 \%$ of patients with PMR have GCA 3 . 


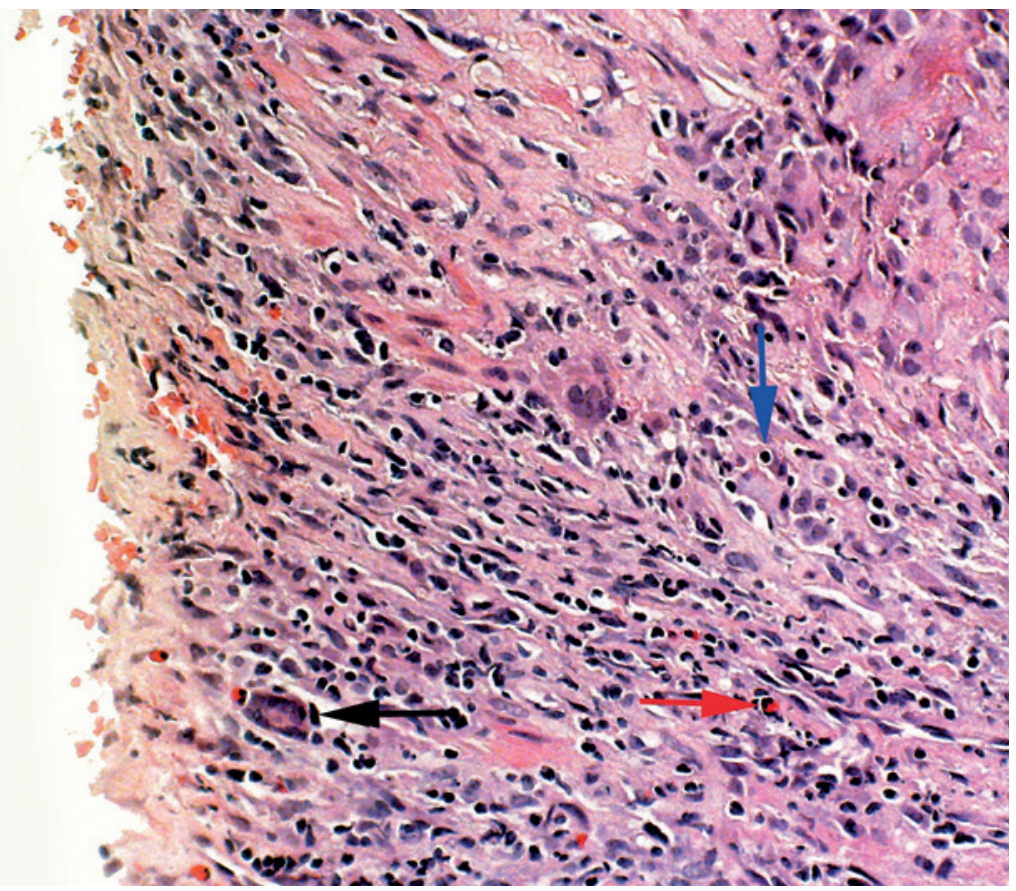

Figure 1 Inflammatory cells.

Lymphocytes (blue arrow), eosinophils (red arrow), and macrophages and giant cells (black arrow) (haematoxylin and eosin preparation, original magnification $\times 100$ ).

Of note, PMR in this patient resolved after statin therapy was discontinued and PMR and GCA recurred after statin therapy (that is, rosuvastatin) was resumed. This sequence of events suggests that statins may trigger PMR and GCA in sensitive patients. However, this patient relapsed later without a preceding statin challenge, showing that the disease process had become independent of the external trigger, which often occurs in drug-induced vasculitis ${ }^{4}$.

The mechanism of statin-associated PMR and GCA is not clear. Because many patients receiving statins develop myalgia and/or more severe muscle disease ${ }^{1}$, mild myopathic damage probably plays a role in statin-associated PMR and GCA. In statinassociated necrotising myopathy, these drugs have recently been postulated to induce neo-antigens as a result of muscle damage, which are subsequently presented to the immune system. A similar mechanism may operate in statin-associated PMR and GCA 2. Indeed, we recently showed in an animal model that statin use leads to dysregulation of immune responses, possibly resulting in autoimmunity 5 . In summary, we report the first case to our knowledge of GCA combined with PMR that was 


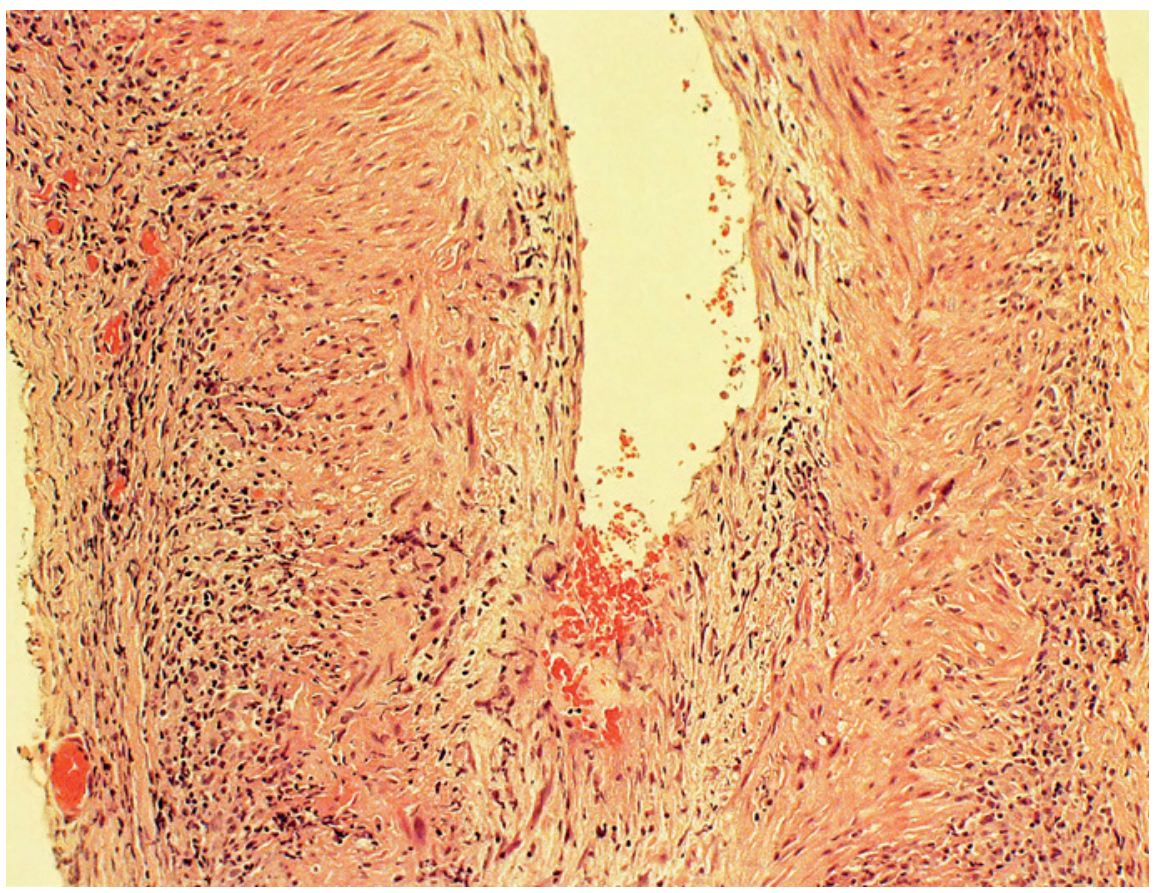

Figure 2 A pan-arteritis of the temporal artery (haematoxylin and eosin preparation, original magnification $\times 200$ )

probably induced by a statin, rosuvastatin. Of interest, in this patient, previous use of atorvastatin had been followed by a first episode of PMR. We propose that PMR should be suspected when a patient receiving statin therapy presents with myalgia of the hips and shoulders combined with morning stiffness. 


\section{REFERENCES}

1. Rallidis LS, Fountoulaki K, Anastasiou-Nana M. Managing the underestimated risk of statin-associated myopathy. Int J Cardiol 2012; 159(3): 169-76.

2. De Jong HJ, Saldi SR, Klungel OH, et al. Statin-Associated Polymyalgia Rheumatica. An Analysis Using WHO Global Individual Case Safety Database: A Case/Non-Case Approach. PLoS One 2012; 7(7): e41289

3. Salvarani C, Cantini F, Hunder GG. Polymyalgia rheumatica and giant-cell arteritis. Lancet 2008; 372(9634): 234-45.

4. Slot M, Links T, Stegeman C, Tervaert JWC. Occurrence of antineutrophil cytoplasmic antibodies and associated vasculitis in patients with hyperthyroidism treated with antithyroid drugs: A long-term followup study. Arthritis rheumatism 2005; 53(1): 108-13.

5. Vandebriel RJ, De Jong HJ, Gremmer ER, et al. Statins accelerate the onset of collagen type II-induced arthritis in mice. Arthritis Res Ther 2012; 14(2): R90. 


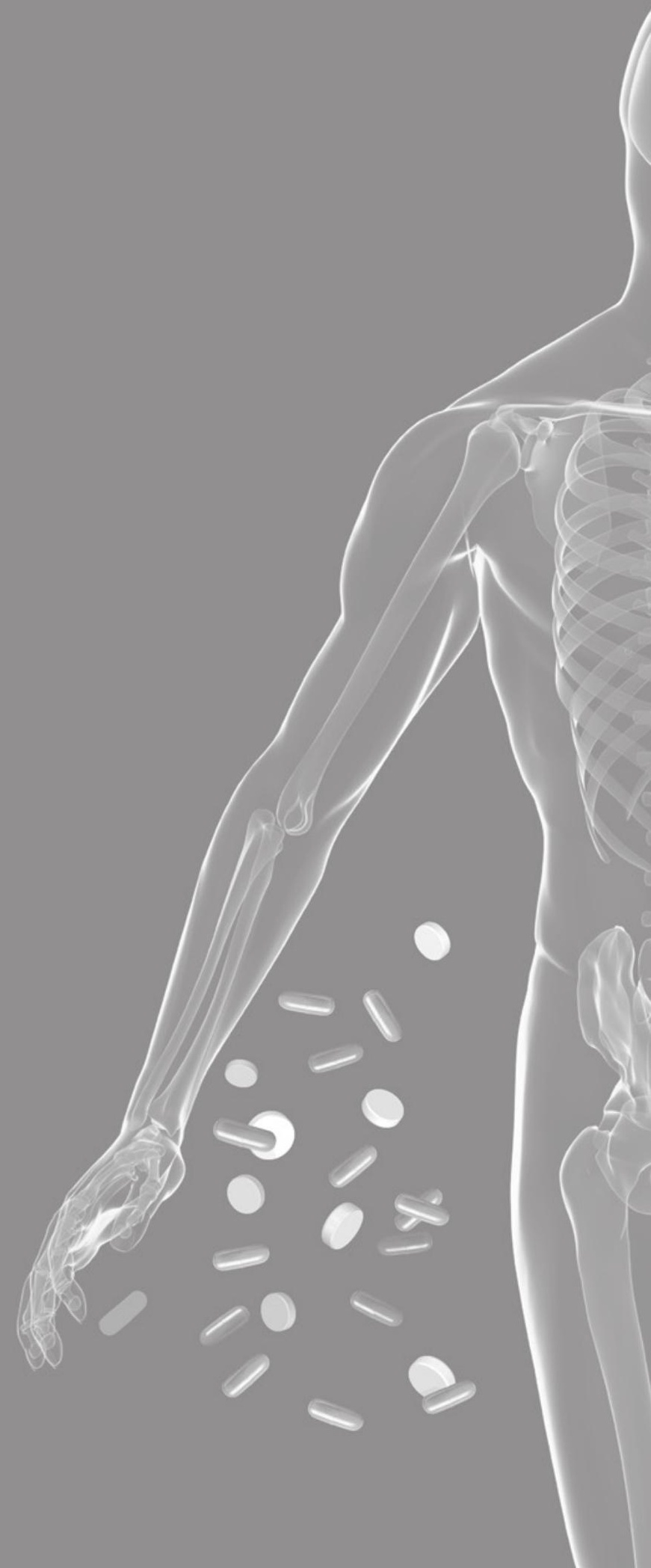




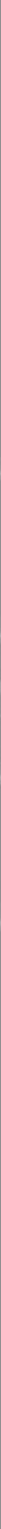




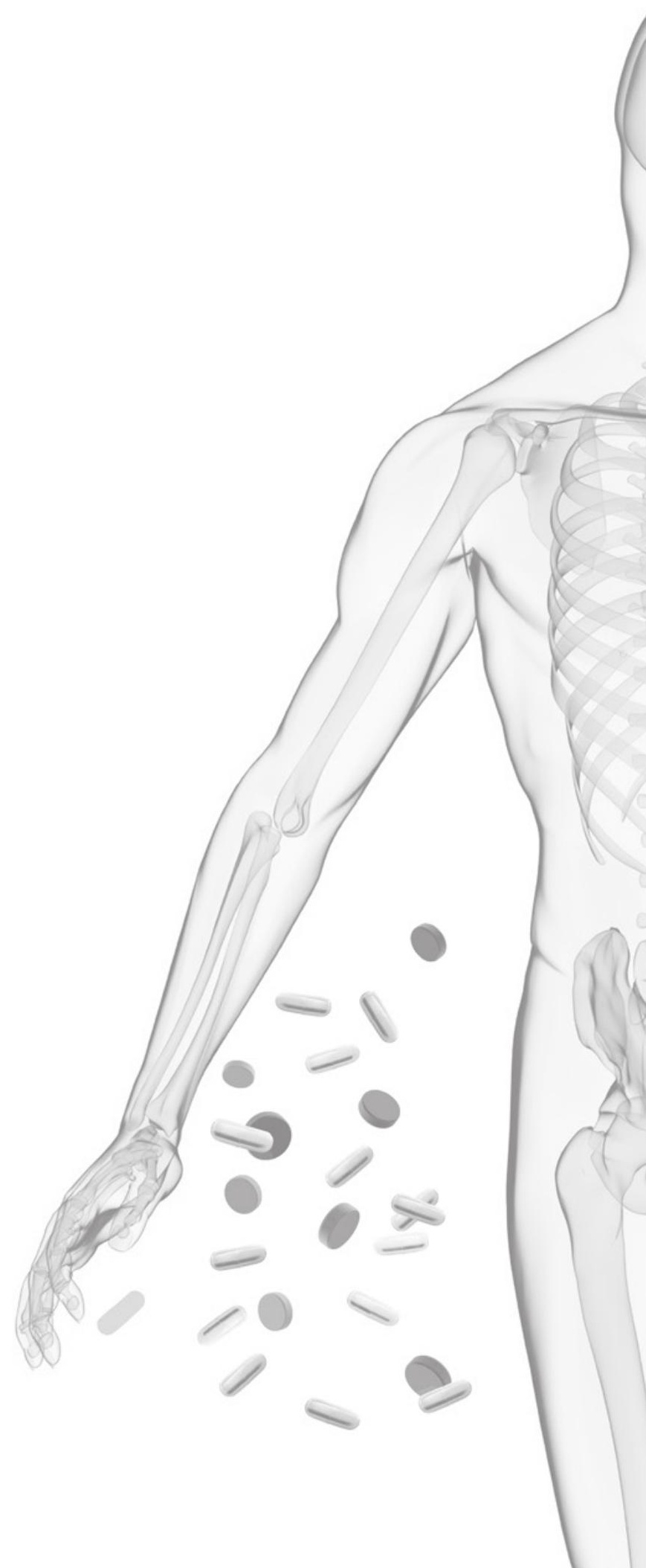





\section{ABSTRACT}

\section{Objectives}

Statins offer significant cardiovascular benefits. Their use, however, influences immune regulation, which may potentially facilitate autoimmunity, eventually resulting in autoimmune diseases such as rheumatoid arthritis (RA). The authors studied whether statin use was associated with an increased risk of developing RA by conducting a case-control study using the Netherlands Information Network of General Practice database.

\section{Methods}

The authors identified 508 patients aged 40 years or older with a first-time diagnosis of RA in the period 2001-2006. Each RA case was matched to five controls for age, sex and index date, which was selected one year before the first diagnosis of RA. Odds ratios for the first-time diagnosis of RA were verified by a referral to a rheumatologist and/or at least one prescription of disease-modifying anti-rheumatic drugs and/or two prescriptions of corticosteroids after the date of first diagnosis.

\section{Results}

Cases were more often users of statins (15.9\%) compared to controls (8.6\%). After adjustment for cardiovascular risk factors and use of co-medication, statin use was associated with an increased risk of incident RA (adjusted OR, 1.71 (95\% Cl 1.16 to 2.53); $p=0.007$ ). A consistent trend of increasing risk with increased cumulative duration, cumulative defined daily doses and number of prescriptions was not observed. However, a small trend between the potency of statin treatment and the risk of RA was found.

\section{Conclusions}

Statin use seems to be associated with an increased risk of developing RA. Our findings should be replicated by additional studies. 


\section{INTRODUCTION}

Epidemiological studies have shown an increasing prevalence of autoimmune diseases in highly industrialised countries, which cannot be attributed to better diagnosis alone but may be due to increased drug use 1,2. Concerns about preventive therapies such as statins, which are widely prescribed to reduce the risk of cardiovascular morbidity and mortality in patients with hyperlipidaemia, hypertension or diabetes, have been raised $3-5$. Several studies have shown that statins, in addition to their cholesterollowering activity, have anti-inflammatory and immunomodulatory properties, suppressing the expression of ongoing autoimmune responses 6-11. Such immunomodulating effects may hypothetically also facilitate the development of autoimmunity, potentially resulting in autoimmune diseases such as systemic lupus erythematosus and autoimmune hepatitis 12,13. Recently, a meta-analysis showed an association between statins and an increased risk of diabetes ${ }^{14}$. As no distinction between the types of diabetes was made, no conclusions on mechanisms could be drawn. Several studies demonstrated that statins may exacerbate or trigger cellular apoptosis 12,15,16 and induce a shift in T helper (Th) 1/Th2 balance, leading to production of autoantibodies 12,17. An increasing number of case reports suggest that statins can trigger rare autoimmune diseases, raising the question on whether these commonly prescribed drugs may facilitate the development of more common autoimmune diseases such as rheumatoid arthritis (RA). So far, few studies have assessed the risk of developing RA after statin treatment. A population-based case-control and cohort study reported that statins may be protective against the development of RA in patients with hyperlipidaemia, 18,19 while two other population-based cohort studies did not observe an association between statin use and incident RA 20,21. We conducted a case-control study to determine the risk of developing RA associated with different exposure aspects (duration, dose, type and potency) of statin use.

\section{METHODS}

\section{Study population}

We used the Netherlands Information Network of General Practice (LINH), a database derived from general practices that register data on morbidity, drug prescriptions and referrals in electronic medical records on a continuous basis. The LINH network includes 350000 patients who were registered at 85 practices in 2001-2006 22. For a number of practices, data on drug prescriptions were not available before the year 2001. Prescription data were classified according to Anatomical Therapeutic and Chemical (ATC) classification, 23 and morbidity was coded using the International Classification of Primary Care (ICPC) 24. 


\section{Case definition}

All patients who were first diagnosed as having RA and had a medical history of at least one year at index date were included in the study (figure 1). Subsequent diagnoses of RA were disregarded. The date exactly one year before the date of the first-time diagnosis of RA was subsequently used as the 'index date' because the diagnosis is usually made after the disease has been symptomatic for some period of time 25,26 . From the arthritis group (ICPC code L88), we verified the first-time diagnosis of RA registered by a general practitioner (GP) if:

1. The patient was referred to a rheumatologist; or

2. At least one disease-modifying anti-rheumatoid drug (DMARD; immunosuppressants, aminoquinolines, gold preparations and sulfasalazine) was prescribed after the index date; or

3. Two or more prescriptions of systemic corticosteroids (glucocorticoids and combinations) were distributed after the index date. Since statins are widely prescribed for patients with cardiovascular diseases or cardiac risk factors that are more prevalent among older patients, patients younger than 40 years of age were excluded from the study. Furthermore, patients were excluded if they had a medical record of ankylosing spondylitis (ICPC code L88.2) or were taking at least one prescription of DMARDs before the index date, if they had been diagnosed by the GP as having RA with no confirmation according to the above-mentioned criteria or if they had no registered medical history for one year.

\section{Control selection}

Five controls were matched to each RA case on age (within five years), sex and index date. Controls were required to be registered in general practice for at least one year before the index date to minimise information bias. The exclusion criteria used in case selection were applied to controls.

\section{Definition of exposure and potential confounders}

Exposure to statins was defined as the use of any approved and commercially available statins (pravastatin, simvastatin, cerivastatin, atorvastatin and fluvastatin) in the Netherlands before the index date. The hazard function between statin use and RA is unknown; therefore, different aspects of statin use were defined. Statin users were patients who had received at least one prescription of statins before the index date. We determined the type of statins based on their last prescription before the index date. The potency of statins was determined by combining the type and the dose of statin into a single potency score to control for the fact that different types and doses of statins differ with respect to percentage reduction in total cholesterol (see online supplementary table 1) 27. Potency was divided into four categories of increasing potency: potencies 2 and 3, potency 4, potency 5, and potencies 6 and 7 . 


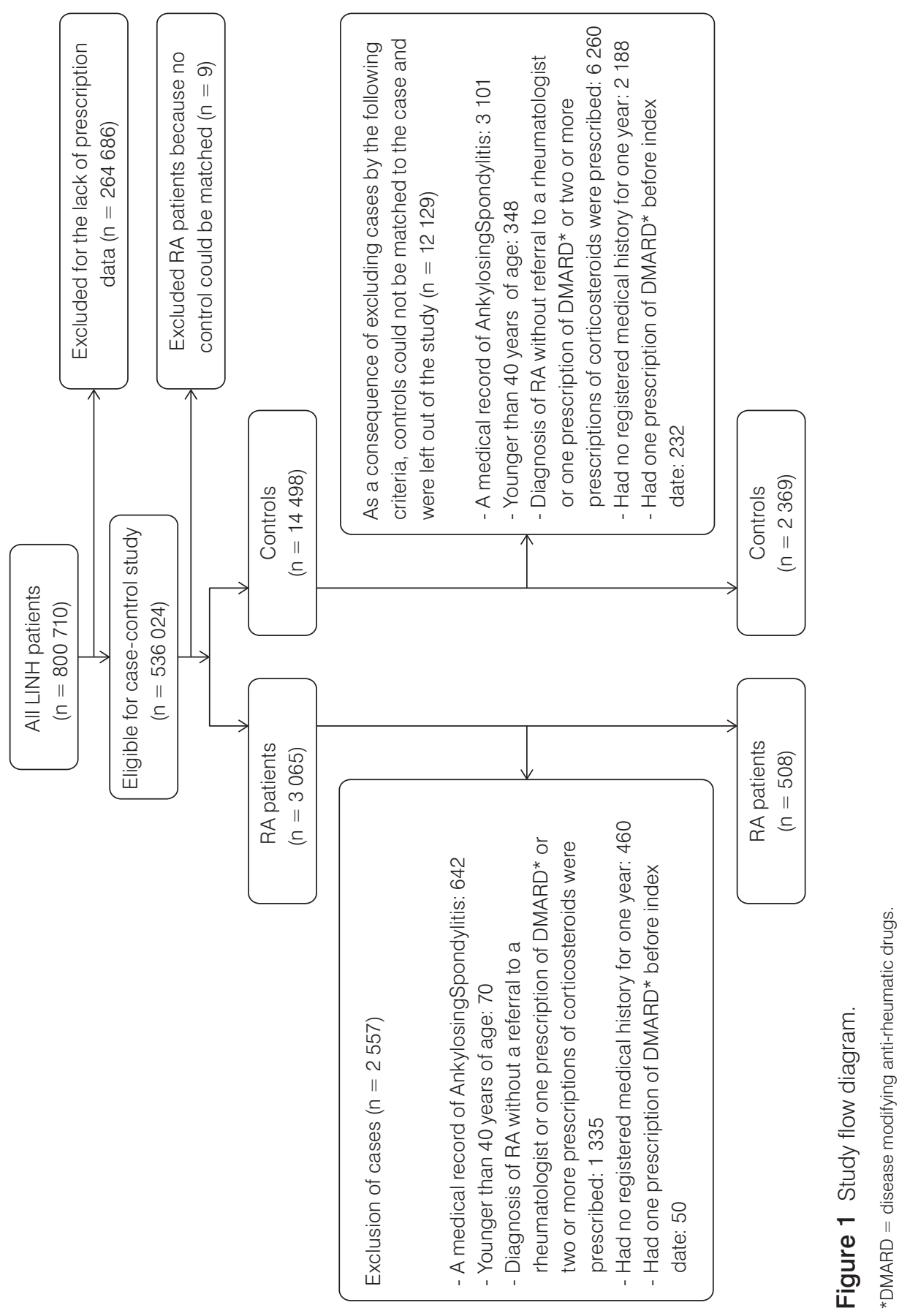


The expected duration of statin use until the index date was based on treatment time and prescribed drug supply (determined by the number of prescribed tablets). We calculated the cumulative dose of statins according to defined daily dose (DDD), which is the assumed average daily dose of a drug for its main indication in adults (see online supplementary table 2) ${ }^{23}$. Adherence to statin use was calculated by dividing the sum of the days' supply by the total number of days between the first prescription and the last prescription of statins in the year before the index date, multiplied by $100 \%$. To determine adherence to statin use, we excluded patients who received one prescription of statins and did not use statins one year before the index date. Patients who received one prescription of statins before the index date were not excluded from other analyses. Potential confounders included prescriptions (in the six months before the index date) for non-steroidal anti-inflammatory drugs (NSAIDs), corticosteroids, proton pump inhibitors, aspirin, antibiotics and hormone replacement treatment, and co-morbidity (diabetes, hyperlipidaemia, hypertension, cardiovascular disease and asthma) ${ }^{28}$. The ICPC and ATC codes for co-morbidity are presented in table 1.

\section{Statistical analysis}

Logistical regression conditioned on matching factors was used to study the association between statin use and risk of RA. Odds ratios and $95 \% \mathrm{Cl}$ were estimated. We evaluated the effects of cumulative duration, cumulative DDDs, number of prescriptions, and the specific type and potency of statins on the risk of developing RA. In addition to controlling for age, sex and calendar time by matching, we adjusted the estimates for the above-mentioned confounders. Furthermore, we evaluated the confounding effects of asthma, aspirin and antibiotics, but we did not include these covariates in the regression models because we did not observe more than $10 \%$ differences in the estimate of exposure-outcome association by adding each time one of these three variables into the model 29 . We examined whether there was a linear trend across the categories of the potencies of statins by including the categorical variables as ordinal variables in the regression model. $P$ values of $\leq 0.05$ were considered statistically significant. We used SAS V.9.1 (SAS Institute, Cary, North Carolina, USA) to analyse the data.

We performed 11 different sensitivity analyses to test the robustness of our findings, which are presented online (see online supplementary table 3). 


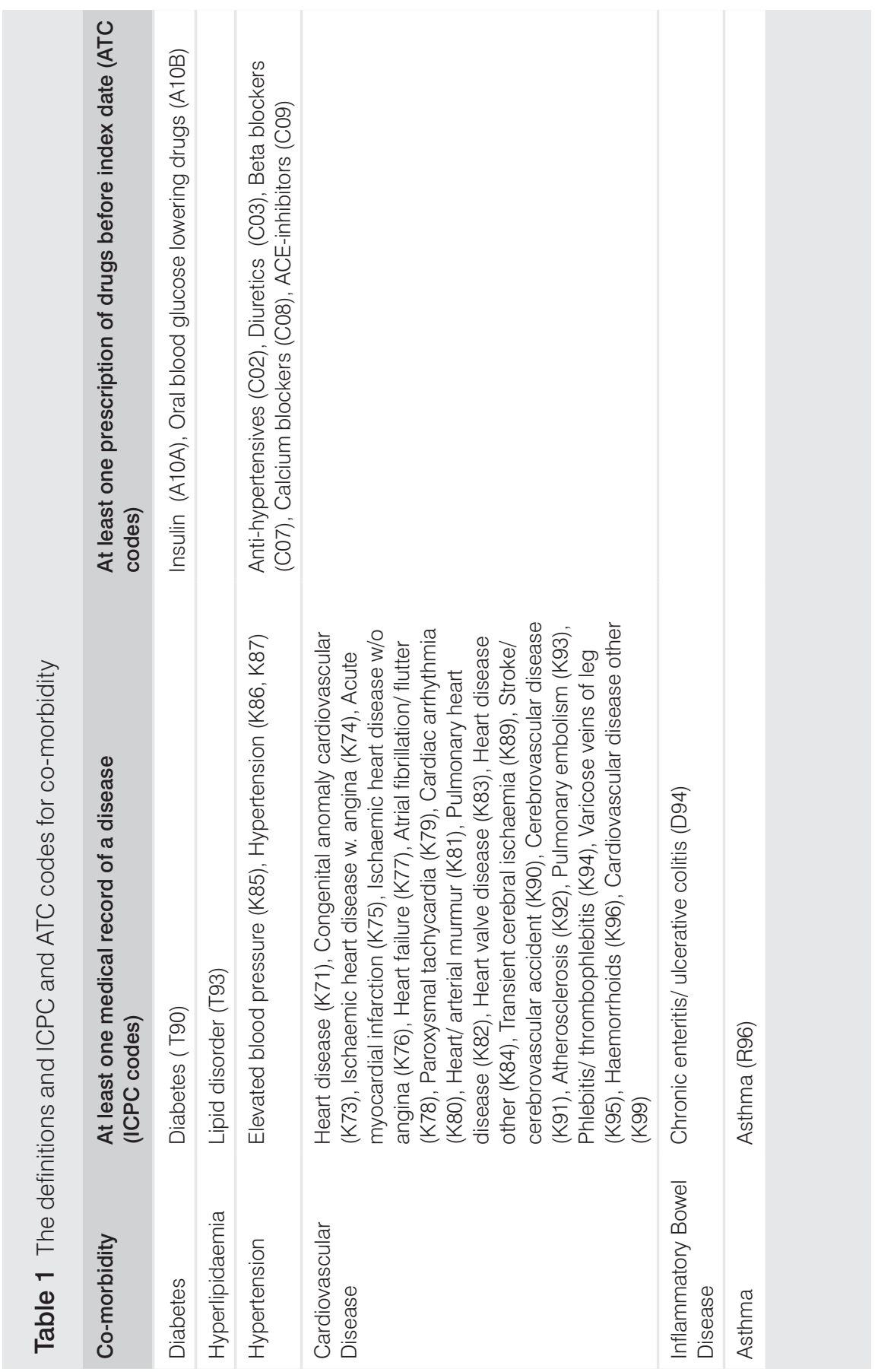




\section{RESULTS}

In the study population, 3074 patients with a first-time diagnosis were identified, of whom nine patients did not have a matched control and 642 patients had a medical record of ankylosing spondylitis. Of 2423 patients with RA, 848 patients were referred to a rheumatologist, 425 patients received at least one prescription of a DMARD and 817 patients were treated with two or more prescriptions of systemic corticosteroids after the index date. Regarding the 848 patients who were referred to a rheumatologist, 155 patients were treated with a DMARD after the index date. As a result of verifying the first-time diagnosis of RA, we identified 508 eligible cases that were matched to 2 369 controls. Of the patients with RA, the average age was 63 years, approximately $67 \%$ of the patients were female and $3 \%$ of the patients were diagnosed as having psoriasis. Fourteen percent of the patients with RA were users of statins. Two patients with RA used other lipid-lowering medications. Use of corticosteroids and NSAIDs was higher in patients with RA than in controls. The duration of registration with the GP was approximately 11.3 years in patients with RA and 10.3 years in controls. The baseline characteristics of the study population are presented in table 2 .

Table 3 shows the results of analyses with different exposure aspects of statin use. Statin use was associated with an increased risk of developing RA (adjusted OR, 1.71 (95\% $\mathrm{Cl} 1.16$ to 2.53); $p=0.007$ ). Regarding the number of prescriptions, an increased risk of developing RA was found for all categories of statin use compared to non-users. No trend between an increasing number of prescriptions and an enhanced risk of developing RA was observed. We explored the effect of the duration of statin use. After adjustment, an increased risk of developing RA was found for different categories of the duration of statin use. No clear relation between the duration of statin use and an increased risk of developing RA was observed. When data were analysed for different levels of DDD, we found no trend between the different DDD groups of statins and the risk of developing RA. Regarding confounding effects on the different strata of DDD, duration of statin prescriptions and number of statin prescriptions, the association between statin use and the risk of developing RA was weaker compared to the result of our overall analysis with ever use of statins ${ }^{30}$. Categorising exposure according to level of adherence did reveal differences in effect. Only patients with an adherence rate of more than $80 \%$ had an increased risk of incident RA. The association between individual statins, their potencies and the risk of RA is shown in table 4.

All types and potencies of statins showed an enhanced risk of incident RA. Regarding the potency of statin treatment, we observed a small trend between the different categories of the potencies of statin treatment and the risk of RA ( $p$ for trend $<0.01$ ). The results of eleven different sensitivity analyses are presented online (see online supplementary table 3). 
Table 2 Baseline characteristics of the study population before index date

\section{Baseline Characteristics}

Male, (\%)

Female, (\%)

Mean age, y (SD)

Duration registration at general practice in years, $(\%)^{\star}$

$1-5.99$

$6-11.99$

$\geq 12$

Disease history before index date (\%)

\section{Diabetes}

Hypertension

Cardiovascular disease

Hyperlipidaemia

Hepatic disease

Renal disease

Asthma

Cancer

Inflammatory bowel syndrome

Psoriasis

Drug use six months before index date (\%)

Corticosteroids

NSAIDs ${ }^{\dagger}$

Statins

Other lipid-lowering agents

Anti-hypertensive agents

Anti-diabetic agents

Aspirin

$\mathrm{HRT}^{\ddagger}$

Antibiotics

Vaccines

Anti-psychotics

Antidepressants

PPIs§

\section{Case \\ $(\mathrm{n}=508)$ \\ Control \\ ( $n=2369)$}

$165(32.5)$

$810(34.2)$

$343(67.5) \quad 1559(65.8)$

$63.4(12.9) \quad 62.8(12.8)$

$118(24.3)$

487 (23.5)

174 (34.3)

800 (33.8)

194 (38.2)

790 (33.3)

$\begin{array}{rc}44(8.7) & 167(7.1) \\ 230(45.3) & 724(30.6) \\ 125(24.6) & 330(13.9) \\ 28(5.5) & 89(3.8) \\ 10(2.0) & 16(0.7) \\ 8(1.6) & 13(0.6) \\ 44(8.7) & 57(2.4) \\ 13(2.6) & 71(3.0) \\ 4(0.8) & 7(0.3) \\ 16(3.2) & 33(1.4)\end{array}$

68 (13.4)

$145(28.5)$

$70(13.8)$

$2(0.4)$

$182(35.8)$

$34(6.7)$

$38(7.5)$

31 (6.1)

81 (15.9)

76 (14.9)

$3(0.6)$

$44(8.7)$

$72(14.2)$
46 (1.9)

$121(5.1)$

$163(6.9)$

$14(0.6)$

$530(22.4)$

$115(4.9)$

105 (4.4)

$62(2.6)$

$199(8.4)$

266 (11.2)

$19(0.8)$

$89(3.8)$

85 (3.6)

* Duration of registration with a general practitioner was defined by the date of registry at a general practice or the first medical record of the patient. No data on date of registry at a general practice before the entrance of this study were available for 314 patients.

† NSAIDs = Nonsteroidal anti-inflammatory drugs

$\ddagger \mathrm{HRT}=$ Hormone replacement therapy

$\S$ PPIs $=$ Proton pump inhibitors 


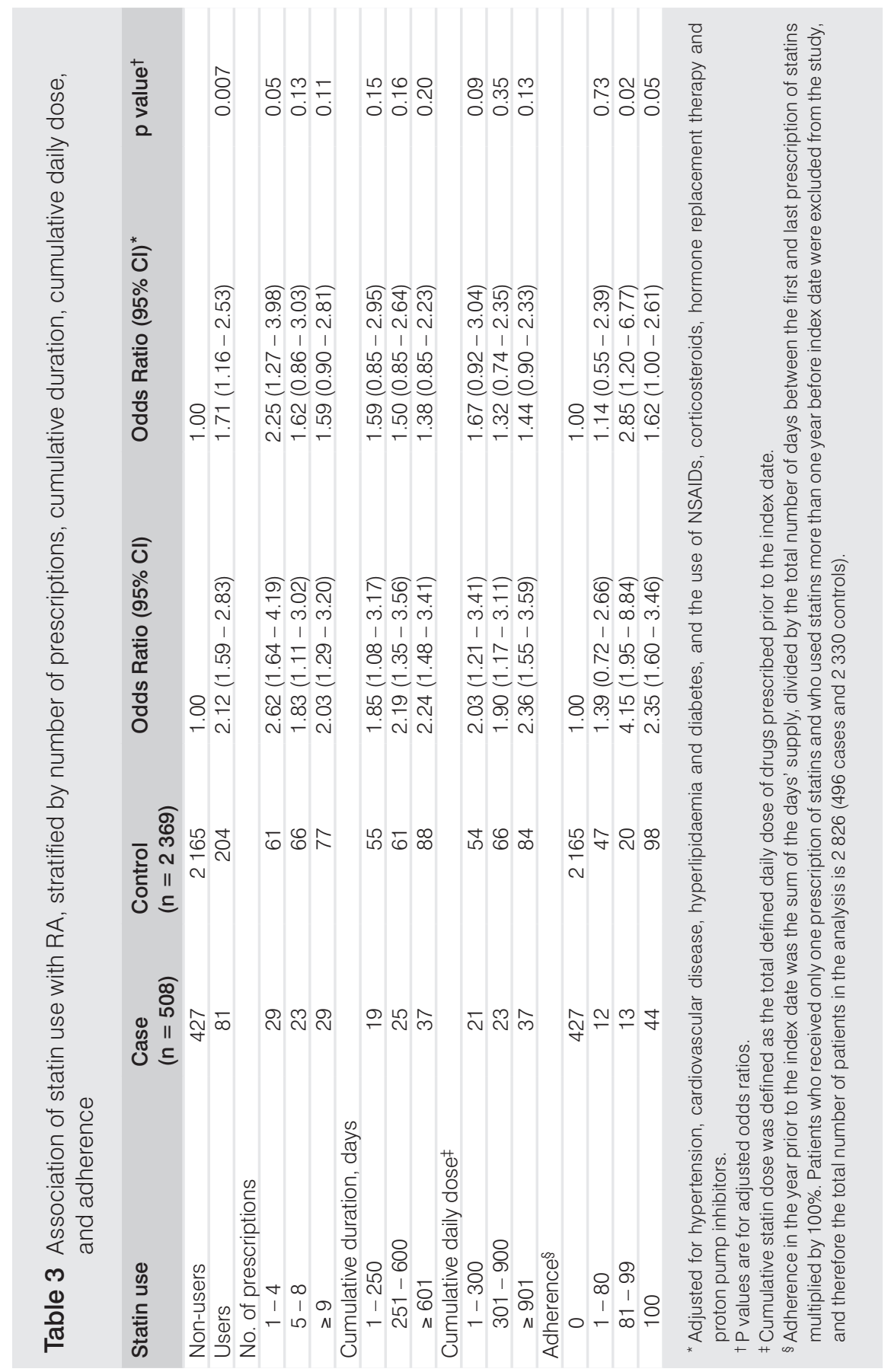




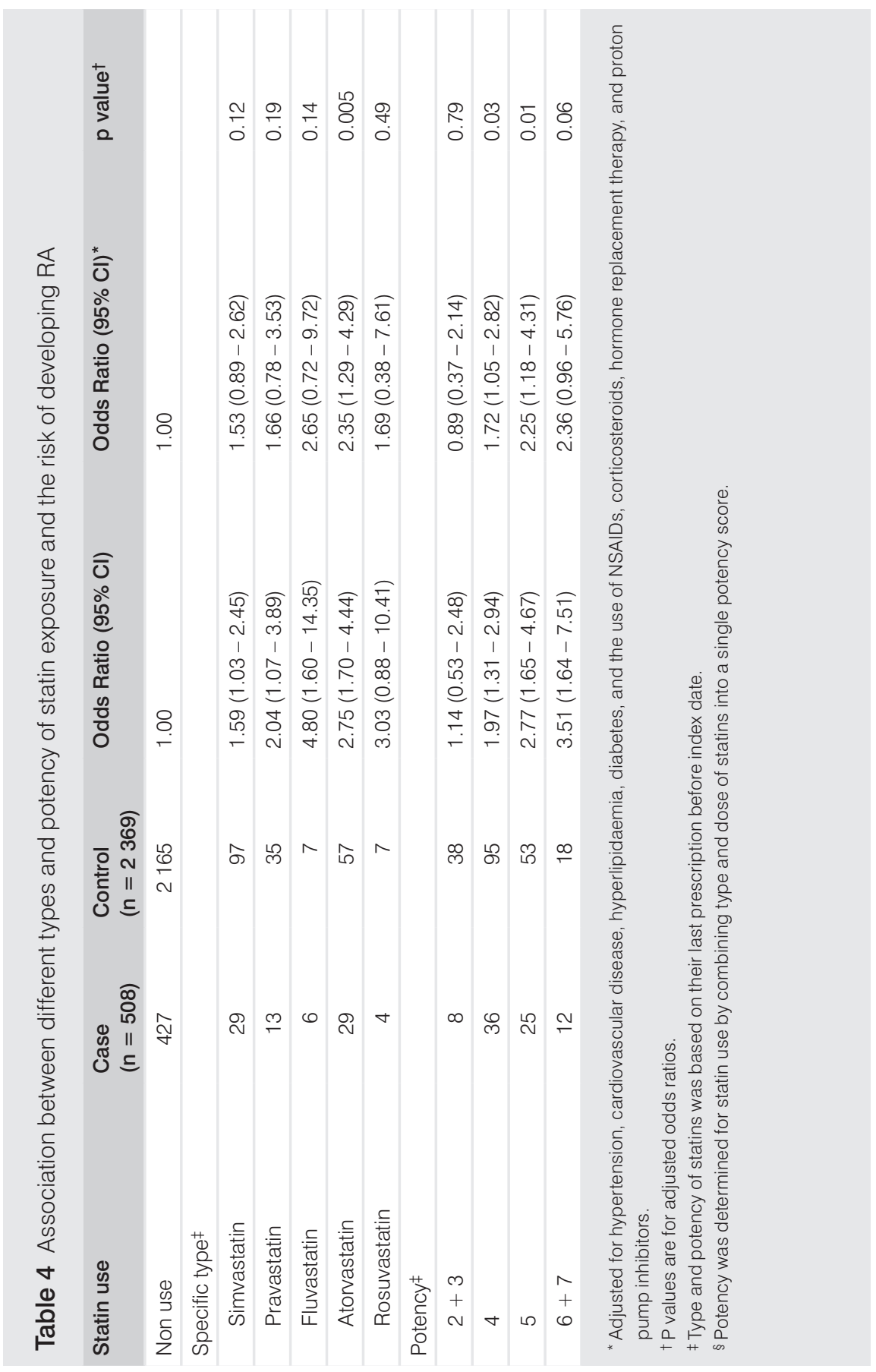




\section{DISCUSSION}

The findings were consistent with the hypothesis that statin use is associated with an increased risk of developing RA. We did not observe a consistent trend of increasing risk with increased cumulative duration, increased cumulative DDDs and increased number of prescriptions. However, we observed a small trend between the potency of statin treatment and the risk of RA. With our baseline characteristics taken into account, cardiovascular diseases and cardiovascular risk factors (e.g. hyperlipidaemia) may have influenced our results. Our hypothesis was made a priority for this study and was supported by several case reports describing the occurrence of autoimmune diseases during treatment with statins 12,13. Our findings are not in line with previous studies, which found that statins exert a protective effect or no effect on developing RA 18-21. In a nested case-control study of 313 patients with incident RA and 1252 matched controls aged 40 to 89 years, current statin use was associated with a reduced risk of developing RA in patients with a diagnosis of hyperlipidaemia, whereas no association was found for patients without a diagnosis of hyperlipidaemia ${ }^{18}$. Because of limited data on hyperlipidaemia in our study, we analysed the data of patients without a medical record for cardiovascular disease, diabetes and hypertension, and we still found an increased risk of developing RA associated with statin use. Recently, three population-based cohort studies of patients who were initially treated with statins have shown conflicting results ${ }^{19-21}$. Smeeth et al.21 reported no effect of statins on the development of RA, whereas Chodick et al. ${ }^{19}$ reported a reduction in the risk of developing RA when patients were treated with statins. Both the study of Smeeth et al. ${ }^{21}$ and the study of Chodick et al. ${ }^{19}$ excluded patients with RA in the first year after the index date. In contrast to our retrospective study, these two prospective studies hypothetically considered a lag time between exposure to statins and incident RA. This could explain the differences between these two cohort studies and our results because we found an increased risk of RA within six months of statin use. Another prospective population-based cohort study of more than two million patients from 368 general practices in England and Wales showed no association between statin use and the risk of developing RA 20. In comparison with this population-based cohort study, we used a more sensitive definition of RA by verifying the patient electronic records with a referral to a rheumatologist and/or at least one DMARD prescription and/or two corticosteroid prescriptions after the date of the first diagnosis of RA. In the study of Hippisley-Cox and Coupland, 20 patients were included as cases if they only had a diagnostic code for RA. Using a more inclusive definition of RA may have diluted the association between statin use and incident RA.

The underlying mechanisms by which statins may facilitate RA are unknown, and it was not possible to investigate them in our study. Statins are suggested to have a 
direct immunomodulating effect on $T$ cells that may promote a shift in Th1/Th2 balance, leading to production of autoantibodies 12,17 . Statins may affect regulatory $T$ cells that are critical for maintaining peripheral tolerance and preventing the development of RA 31-33. Recent studies have suggested that regulatory T cells can be unstable in the periphery and may promote autoimmunity 34,35 . According to these studies, we hypothesise that statins do not cause autoimmunity but may promote a pre-existing autoimmune-prone condition to progress towards a clinical disease such as RA. Another possibility is that self-tolerance is lost due to non-specific bystander activation provided by local inflammation (microbial infection), which could result in the formation of neo-antigens 36 . This mechanism may be induced by statins that have been proven to reduce Th1 responses 8 .

One of the strengths of our study is the use of a computerised database, allowing us to use routinely recorded medical and prescription data from GPs. Consequently, recall bias was minimised. Our study contains a relatively large number of patients with RA, which allowed an assessment of the association between statins and RA with sufficient precision. Finally, we performed a range of sensitivity analyses regarding outcome definition, exposure definition, and inclusion and exclusion criteria. All these analyses consistently have shown an increased risk of incident RA associated with statin use.

Some limitations of this study should be considered. A concern may be the power of this study; therefore, we used a definition of RA that is based on GP diagnosis, a referral to a rheumatologist or prescriptions of DMARDs or corticosteroids after the index date. Thus, we may have used a relatively sensitive but non-specific diagnosis of RA because no specific ICPC codes were available for other rheumatic diseases (e.g. systemic lupus erythematosus and psoriatic arthritis) that may have attenuated the association between statins and RA. However, our results remained unchanged when the analyses were restricted to patients with RA who were referred to a rheumatologist or who received DMARDs after their first-time diagnosis, or when we excluded from the analysis patients with a diagnosis of psoriasis or patients who used corticosteroids and NSAIDs before the index date. In addition, we have attempted to verify the first-time diagnosis of RA by the GP: if patients were referred to a rheumatologist and received at least one DMARD prescription after the index date, or if patients were prescribed DMARDs after the index date. Using these two criteria for defining RA, we have only included patients who were referred to a rheumatologist and were prescribed DMARDs by their GP, or patients who were treated with DMARDs by their GP. When DMARDs were prescribed by the rheumatologist, the prescription of the patient may not always appear in the LINH data set. The LINH database does not provide medical information from the rheumatologist, but the Dutch guidelines for optimising GP-medical specialist communication enjoin medical specialists to inform GPs of the first results of the 
diagnostics and treatments of the referred patient ${ }^{37,38}$. Due to this possible underestimation of patients with RA, we may have introduced selection bias that may produce an underestimation of the association. Using computerised prescription data from the GP may have introduced an overestimation of actual statin use. However, this misclassification is likely to be non-differential between cases and controls and, therefore, would probably have resulted in an underestimation of the association between statin use and RA. In this study, we found an effect of statin use on the risk of developing RA. We observed no effect of past statin use and no trend between different groups of duration, DDDs and prescriptions of statins and the risk of developing RA. This may be due to the small number of patients in the study. There were no available data on dietary intake, physical activity and smoking, and there were limited available data on other examinations (e.g. lipid levels, inflammatory and immunomodulatory markers (e.g. anti-nuclear antibodies, anti-citrullinated protein antibodies)) that may be important confounders. By conducting simulation analyses with potential effects of smoking, ${ }^{39}$ we estimated the impact of smoking on the association between statin use and the development of RA 40 .Our findings were similar after adjusting for smoking. Nonetheless, we cannot exclude the possibility of residual confounding.

Cardiovascular morbidity and mortality are enhanced in patients with RA, and this could be due to the inflammatory process and the increased prevalence of traditional cardiovascular risk factors such as hyperlipidaemia 41-43. Several studies have demonstrated an unfavourable lipid profile in patients with RA 41,44. Therefore, statin use could be a proxy for hyperlipidaemia, which may be responsible for an increased risk of developing RA in our study. With our baseline characteristics taken into account, cardiovascular diseases and cardiovascular risk factors (e.g. hyperlipidaemia) may have influenced our results. Therefore, we should consider that the increased risk of RA was due to hyperlipidaemia and not due to statins. Conversely, several studies did not find significant differences between the lipid profile of patients with RA and the lipid profile of the general population ${ }^{45-47}$.These conflicting results can be attributed to inflammation and treatment with lipid-lowering drugs 45,46,48. Myasoedova et al. 45 reported that reduction of lipid levels in patients with RA is unlikely to be solely due to lipid-lowering treatment. Additionally, confounding by indication may have affected the results of this study. We have limited information on hyperlipidaemia due to under-registration of this diagnosis in the GPs' records. Therefore, to minimise confounding by indication, we conducted a subgroup analysis based on cardiovascular risk profile. Statin use was associated with an increased risk of incident RA in patients with a low cardiovascular risk profile. With the potency of statins taken into account, certain assumptions about disease severity in the patients in our study can be made. We believe that a high potency of statins is related to severe cardiovascular disease/hyperlipidaemia. Based on this assumption, we would 
expect the association between the increased potency of statin treatment and the risk of developing RA to disappear or to be much more diminished among those cases with severe cardiovascular disease and/or hyperlipidaemia. However, we observed a small trend between an increased potency of statin treatment and an increased risk of RA. Taken together, it is not very likely that hyperlipidaemia is the only risk factor. Selection bias might have been introduced in this study because GPs could have paid more attention to the comorbidities of patients with RA. Consequently, these patients may have an increased probability of receiving statins. A sensitivity analysis in which we included controls who were registered and visited the GP showed similar results. Additionally, the average duration of registration with the GP is almost equal between patients with RA and controls, indicating that the patients in our study had similar opportunities to have been prescribed a statin. Differences between GPS could have biased the results of our study if there are differences in diagnoses and prescribing regimens per practice. However, because of the small number of patients with RA per GP, we did not control for general practice effects in our analysis. It is likely that statins were prescribed before the first-time diagnosis of RA. In our study, we could define the date of onset of RA by the first record of the GP for RA, but the date of onset of the RA symptoms is unknown ${ }^{49}$. Chan et al. 25 reported that the median time between the onset of symptoms and the diagnosis of RA was less than one year. To prevent this possible type of bias (protopathic bias) of defining the date of onset, we performed several sensitivity analyses by modifying the index date exactly two, three and four years before the first-time diagnosis of RA. Our results remained unchanged in all sensitivity analyses. Because of the small sample size of the study population, we used an index date exactly one year before the first-time diagnosis of RA. From approximately 500000 eligible patients, we ultimately included 508 patients with RA in our study. Our restricted sample of patients with RA may hamper the external validity of the study.

Our study shows that even if statins are effective in suppressing symptoms of ongoing autoimmunity, they also seem to be associated with an increased risk of developing RA. Our findings should be replicated by additional studies. When confirmed, these findings indicate that precaution should be taken when prescribing statins for individuals with a low risk of cardiovascular disease.

\section{Acknowledgements}

The authors would like to thank the staff of LINH for their help with data collection and data extraction, and $\mathrm{R}$ Janssen for her helpful suggestions. 


\section{REFERENCES}

1. World Health Organisation Collaborating Centre for Immunotoxicology and Allergic Hypersensitivity. Principles and methods for assessing autoimmunity associated with exposure to chemicals: Environmental Health Criteria 236. Bilthoven, The Netherlands: World Health Organisation, 2006.

2. Mongey A, Hess E. Drug insight: autoimmune effects of medications-what's new? Nature clinical practice rheumatology 2008; 4(3): 136-44.

3. Baigent C, Keech A, Kearney PM, et al. Efficacy and safety of cholesterol-lowering treatment: prospective meta-analysis of data from 90,056 participants in 14 randomised trials of statins. Lancet 2005; 366(9493): 1267-78.

4. Colhoun HM, Betteridge DJ, Durrington PN, et al. Primary prevention of cardiovascular disease with atorvastatin in type 2 diabetes in the Collaborative Atorvastatin Diabetes Study (CARDS): multicentre randomised placebo-controlled trial. Lancet 2004; 364(9435): 685-96.

5. Sever PS, Dahlöf B, Poulter NR, et al. Prevention of coronary and stroke events with atorvastatin in hypertensive patients who have average or lower-than-average cholesterol concentrations, in the Anglo-Scandinavian Cardiac Outcomes Trial--Lipid Lowering Arm (ASCOT-LLA): a multicentre randomised controlled trial. Lancet 2003; 361(9364): 1149-58.

6. Ridker PM, Danielson E, Fonseca FA, et al. Reduction in C-reactive protein and LDL cholesterol and cardiovascular event rates after initiation of rosuvastatin: a prospective study of the JUPITER trial. Lancet 2009; 373(9670): 1175-82.

7. McCarey DW, Mclnnes IB, Madhok R, et al. Trial of Atorvastatin in Rheumatoid Arthritis (TARA): double-blind, randomised placebo-controlled trial. Lancet 2004; 363(9426): 2015-21.

8. Arnaud C, Braunersreuther V, Mach F. Toward immunomodulatory and anti-inflammatory properties of statins. Trends Cardiovasc Med 2005; 15(6): 202-6.

9. Kanda H, Yokota K, Kohno C, et al. Effects of low-dosage simvastatin on rheumatoid arthritis through reduction of Th1/Th2 and CD4/CD8 ratios. Mod Rheumatol 2007; 17(5): 364-8..

10. Funk JL, Chen J, Downey KJ, Clark RA. Bone protective effect of simvastatin in experimental arthritis. J Rheumatol 2008; 35(6): 1083-91.

11. Lodi S, Evans SJ, Egger P, Carpenter J. Is there an anti-inflammatory effect of statins in rheumatoid arthritis? Analysis of a large routinely collected claims database. Br J Clin Pharmacol 2010; 69(1): 85-94.

12. Noël B. Lupus erythematosus and other autoimmune diseases related to statin therapy: a systematic review. J Eur Acad Dermatol Venereol 2007; 21(1): 17-24.

13. Alla V, Abraham J, Siddiqui J, Raina D, Wu G, Chalasani N, Bonkovsky H. Autoimmune hepatitis triggered by statins. J Clin Gastroenterol 2006; 40(8): 757-61.

14. Sattar N, Preiss D, Murray HM, et al. Statins and risk of incident diabetes: a collaborative meta-analysis of randomised statin trials. Lancet 2010; 375(9716): 735-42.

15. Mevorach D. Systemic lupus erythematosus and apoptosis: a question of balance. Clin Rev Allergy Immunol 2003; 25(1): 49-60.

16. Chapman-Shimshoni D, Yuklea M, Radnay J, Shapiro H, Lishner M. Simvastatin induces apoptosis of B-CLL cells by activation of mitochondrial caspase 9. Exp Hematol 2003; 31(9): 779-83.

17. Youssef S, Stüve O, Patarroyo JC, et al. The HMG-CoA reductase inhibitor, atorvastatin, promotes a Th2 bias and reverses paralysis in central nervous system autoimmune disease. Nature 2002; 420(6911): 78-84.

18. Jick SS, Choi H, Li L, Mclnnes IB, Sattar N. Hyperlipidaemia, statin use and the risk of developing rheumatoid arthritis. Ann Rheum Dis 2009; 68(4): 546-51.

19. Chodick G, Amital H, Shalem Y, Kokia E, Heymann AD, Porath A, Shalev V. Persistence with statins and onset of rheumatoid arthritis: a population-based cohort study. PLoS Med 2010; 7(9): e1000336.

20. Hippisley-Cox J, Coupland C. Unintended effects of statins in men and women in England and Wales: population based cohort study using the QResearch database. BMJ 2010; 340: c2197.

21. Smeeth L, Douglas I, Hall AJ, Hubbard R, Evans S. Effect of statins on a wide range of health outcomes: a cohort study validated by comparison with randomized trials. Br J Clin Pharmacol 2009; 67(1): 99-109.

22. Linh.nl [internet]. Utrecht: Netherlands Institute for health services research; c1992 - [updated 2010 Feb 23; cited 2010 Mar 4]. Available from: http://www.linh.nl/. 
23. Whocc.no [internet]. Oslo: WHO Collaborating Centre for Drug Statistics Methodology; c1996 - [updated 2010 Feb 5; cited 2010 Mar 4]. Available from: http://http://www.whocc.no/atc_ddd_index/.

24. Lamberts H, Wood M. ICPC. International Classification of Primary Care. Oxford: Oxford Unniversity Press 1987.

25. Chan KW, Felson DT, Yood RA, Walker AM. The lag time between onset of symptoms and diagnosis of rheumatoid arthritis. Arthritis Rheum 1994; 37(6): 814-20.

26. Palm $\varnothing$, Purinszky E. Women with early rheumatoid arthritis are referred later than men. Ann Rheum Dis 2005; 64(8): 1227-8.

27. Penning-van Beest FJ, Termorshuizen F, Goettsch WG, Klungel OH, Kastelein JJ, Herings RM. Adherence to evidence-based statin guidelines reduces the risk of hospitalizations for acute myocardial infarction by 40\%: a cohort study. Eur Heart J 2007; 28(2): 154-9.

28. Pedersen M, Jacobsen S, Klarlund M, Pedersen BV, Wiik A, Wohlfahrt J, Frisch M. Environmental risk factors differ between rheumatoid arthritis with and without auto-antibodies against cyclic citrullinated peptides. Arthritis Res Ther 2006; 8(4): R133.

29. Mickey RM, Greenland S. The impact of confounder selection criteria on effect estimation. Am J Epidemiol 1989; 129(1): 125-37.

30. Greenland S, Robin MR, Pearl J. Confounding and collapsibility in causal inference. Stat Science 1999; 14: 29-46.

31. Kagami S, Owada $\mathrm{T}$, Kanari $\mathrm{H}$, et al. Protein geranylgeranylation regulates the balance between Th17 cells and Foxp3 regulatory T cells. Int Immunol 2009; 21(6): 679-89.

32. Esensten $\mathrm{JH}$, Wofsy D, Bluestone JA. Regulatory T cells as therapeutic targets in rheumatoid arthritis. Nat Rev Rheumatol 2009; 5(10): 560-5.

33. Tang TT, Song Y, Ding YJ, et al. Atorvastatin up-regulates regulatory T-cell and improves clinical disease activity in patients with rheumatoid arthritis. J Lipid Res 2011; 52: 1023-32.

34. Zhou X, Bailey-Bucktrout SL, Jeker LT, et al. Instability of the transcription factor Foxp3 leads to the generation of pathogenic memory T cells in vivo. Nat Immunol 2009; 10(9): 1000-7.

35. Komatsu N, Mariotti-Ferrandiz ME, Wang Y, Malissen B, Waldmann H, Hori S. Heterogeneity of natural Foxp3+ T cells: a committed regulatory T-cell lineage and an uncommitted minor population retaining plasticity. Proc Natl Acad Sci U S A 2009; 106(6): 1903-8.

36. Kivity S, Agmon-Levin N, Blank M, Shoenfeld Y. Infections and autoimmunity--friends or foes? Trends Immunol 2009; 30(8): 409-14.

37. Preston $\mathrm{C}$, Cheater F, Baker R, Hearnshaw H. Left in limbo: patients' views on care across the primary/ secondary interface. Qual Health Care 1999; 8(1): 16-21.

38. Kvamme OJ, Olesen F, Samuelson M. Improving the interface between primary and secondary care: a statement from the European Working Party on Quality in Family Practice (EQuiP). Qual Health Care 2001; 10(1): 33-9.

39. Sugiyama D, Nishimura K, Tamaki K, Tsuji G, Nakazawa T, Morinobu A, Kumagai S. Impact of smoking as a risk factor for developing rheumatoid arthritis: a meta-analysis of observational studies. Ann Rheum Dis 2010; 69(1): 70-81.

40. Lin DY, Psaty BM, Kronmal RA. Assessing the sensitivity of regression results to unmeasured confounders in observational studies. Biometrics 1998; 54(3): 948-63.

41. Van Halm VP, Nielen MM, Nurmohamed MT, et al. Lipids and inflammation: serial measurements of the lipid profile of blood donors who later developed rheumatoid arthritis. Ann Rheum Dis 2007; 66(2): 184-8.

42. Solomon DH, Greenberg J, Reed G, Setoguchi S, Tsao P, Kremer J. Cardiovascular risk among patients with RA in CORRONA: comparing the explanatory value of traditional cardiovascular risk factors with RA risk factors. Ann Rheum Dis 2008; 67(Suppl 2): 482

43. Peters MJ, Nielen MM, Raterman HG, Verheij RA, Schellevis FG, Nurmohamed MT. Increased cardiovascular disease in patients with inflammatory arthritis in primary care: a cross-sectional observation. J Rheumatol 2009; 36(9): 1866-8.

44. Steiner G, Urowitz MB. Lipid profiles in patients with rheumatoid arthritis: mechanisms and the impact of treatment. Semin Arthritis Rheum 2009; 38(5): 372-81.

45. Myasoedova E, Crowson CS, Kremers HM, Fitz-Gibbon PD, Therneau TM, Gabriel SE. Total cholesterol and LDL levels decrease before rheumatoid arthritis. Ann Rheum Dis 2010; 69(7): 1310-4. 
46. Hurt-Camejo E, Paredes S, Masana L, et al. Elevated levels of small, low-density lipoprotein with high affinity for arterial matrix components in patients with rheumatoid arthritis: possible contribution of phospholipase A2 to this atherogenic profile. Arthritis Rheum 2001; 44(12): 2761-7.

47. Goodson NJ, Silman AJ, Pattison DJ, et al. Traditional cardiovascular risk factors measured prior to the onset of inflammatory polyarthritis. Rheumatology (Oxford) 2004; 43(6): 731-6.

48. Libby P. Role of inflammation in atherosclerosis associated with rheumatoid arthritis. Am J Med 2008; 121(10 Suppl 1): S21-31.

49. Vandenbroucke JP. Oral contraceptives and rheumatoid arthritis. Lancet 1983; 2(8343): 228-9. 
Supplementary Table S1 Potency convertibility of statins per dose (in mg)

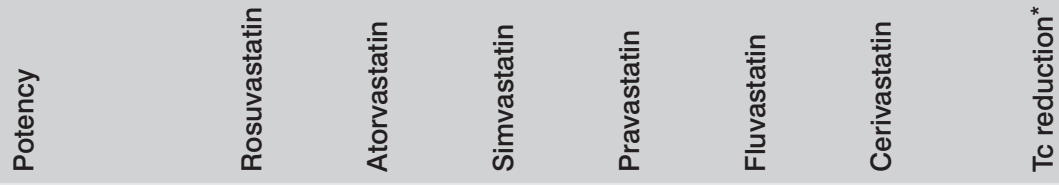

1. Low $6-15 \%$

2. Low

3. Medium

$5 \quad 10$

4. High

$10-20$

20
$15-17 \%$

5. High 10

20

40

40

0.1
$22 \%$

6. High

$10 \quad 20 \quad 40$

80
$27 \%$

7. High

$40 \quad 80$
$32 \%$

${ }^{*} \mathrm{Tc}=$ total cholesterol

\section{Supplementary text S2}

Defined Daily Dose (DDD) of statins according to the guidelines of the WHO Collaborating Centre for Drug Statistics Methodology,

1 DDD corresponds with:
simvastatin $30 \mathrm{mg}$
pravastatin $30 \mathrm{mg}$,
atorvastatin $20 \mathrm{mg}$,
fluvastatin $60 \mathrm{mg}$,
lovastatin $45 \mathrm{mg}$,
rosuvastatin $10 \mathrm{mg}$ 


\section{Supplementary Table S3 Several sensitivity analyses to test the robustness of our findings}

\begin{tabular}{|c|c|c|}
\hline \multirow[t]{2}{*}{ Sensitivity analyses } & \multicolumn{2}{|c|}{ Cases } \\
\hline & $\mathrm{N}$ & Statin use \\
\hline $\begin{array}{l}\text { Restrict to } \mathrm{RA}^{\ddagger} \text { patients referred to a rheumatologist or received at last one } \\
\text { prescription of a DMARD } \text { before index date }^{1}\end{array}$ & 327 & 44 \\
\hline Exclude RA patients with corticosteroid and NSAID" use before index date 2 & 317 & 47 \\
\hline Remove RA patients who had a recorded diagnosis of psoriasis ${ }^{3}$ & 492 & 79 \\
\hline $\begin{array}{l}\text { Define use of statins as } 4 \\
\text { Current statin use } \\
\text { Past statin use }\end{array}$ & 508 & $\begin{array}{l}70 \\
11\end{array}$ \\
\hline $\begin{array}{l}\text { Define current use of statins as receipt of at least two prescriptions within } \\
\text { one year before index date } 5\end{array}$ & 508 & 69 \\
\hline $\begin{array}{l}\text { Modify index date by using the date of the first diagnosis of RA set by the } \\
\text { GP or the date of referral to a rheumatologist } 6\end{array}$ & 508 & 98 \\
\hline \multicolumn{3}{|l|}{$\begin{array}{l}\text { Shift the index date exactly two, three and four years before the first-time } \\
\text { diagnosis of RA7 }\end{array}$} \\
\hline Two years & 370 & 65 \\
\hline Three years & 220 & 43 \\
\hline Four years & 98 & 24 \\
\hline Exclude patients who used statins six months after entrance of the study ${ }^{8}$ & 458 & 31 \\
\hline $\begin{array}{l}\text { Patients without a medical record for cardiovascular disease, diabetes, and } \\
\text { hypertension }{ }^{9}\end{array}$ & 239 & 12 \\
\hline $\begin{array}{l}\text { Exclude patients with a diagnosis for hepatic diseases, asthma and } \\
\text { psoriasis, and antibiotic usage } 10\end{array}$ & 378 & 59 \\
\hline $\begin{array}{l}\text { Exclude subjects who did not have a medical history in the general practice } \\
\text { database } 11\end{array}$ & 508 & 81 \\
\hline
\end{tabular}

* When appropriate, adjusted for hypertension, cardiovascular disease, hyperlipidaemia and diabetes, and the use of NSAIDs, corticosteroids, hormone replacement therapy and proton pump inhibitors.

$\dagger P$ values are for adjusted odds ratios.

$\ddagger R A=$ rheumatoid arthritis

$\S \mathrm{DMARD}=$ Disease modifying anti-rheumatic drug

" NSAID = Non-steroidal anti-inflammatory drugs

"GP = General Practitioner

1 Sensitivity analysis: varying the definition of the outcome (RA), specified as first-time diagnosis of RA and a referral to a rheumatologist and/or a prescription of DMARDs before index date (irrespective of corticosteroids use), a definition used by Jick et al. ${ }^{18}$

2 Sensitivity analysis: estimating the association between statin use and the risk of developing RA by excluding RA patients who use corticosteroids and NSAIDs prior to the index date to exclude any prevalent cases of RA.

3 Sensitivity analysis: investigating the influence of the inclusion of patients with psoriatic arthritis by excluding patients with a medical record for psoriasis.

4 Sensitivity analysis: evaluating the association between current and past use of statins and RA by classifying current use as receiving at least one prescription of statins within six months before index date, and past use as receiving a prescription of statins but discontinued treatment more than six months before index date. 


\section{Controls}

Odds Ratio $(95 \% \mathrm{Cl})$

Odds Ratio $(95 \% \mathrm{Cl})^{*}$

$p$ value ${ }^{\dagger}$

N Statin use

1240

87

$2.12(1.59-3.16)$

$1.97(1.15-3.36)$

0.01

1502

121

$2.06(1.41-3.00)$

$1.72(1.04-2.84)$

0.04

2296

201

$2.09(1.56-2.80)$

$1.54(1.08-2.21)$

0.02

2369

163
44

$163 \quad 2.27(1.66-3.09)$

$44 \quad 1.40(0.71-2.75)$

$1.78(1.18-2.68)$

0.006

$1.28(0.58-2.84)$

0.54

2369

166

$2.19(1.60-2.99)$

$1.71(1.13-2.58)$

0.01

2369

243

$2.20(1.68-2.89)$

$1.44(0.93-2.22)$

0.10

\begin{tabular}{ccccc}
1716 & 161 & $2.16(1.57-2.99)$ & $1.85(1.19-2.87)$ & 0.07 \\
1031 & 99 & $2.43(1.62-3.65)$ & $2.15(1.23-3.74)$ & 0.007 \\
458 & 50 & $2.89(1.63-5.15)$ & $1.64(0.73-3.68)$ & 0.47 \\
\hline 2238 & 74 & $2.11(1.36-3.26)$ & $1.68(0.98-2.91)$ & 0.06 \\
\hline 1515 & 23 & $3.50(1.71-7.14)$ & $2.92(1.33-6.40)$ & 0.008 \\
2081 & 164 & $2.15(1.56-2.98)$ & $1.73(1.12-2.65)$ & 0.01 \\
1940 & 202 & $1.67(1.24-2.18)$ & $1.65(1.13-2.43)$ & 0.01
\end{tabular}

5 Sensitivity analysis: using another definition of current statin exposure that has been postulated by Jick et al., studying the association between current statin use and RA. They defined current use of statins as receipt of at least two prescriptions within one year before index date.

6 Sensitivity analysis: modifying index date by using the date of the first diagnosis of RA set by the GP or the date of referral to a rheumatologist.

7 Sensitivity analysis: shifting the index date exactly two, three and four years before the first-time diagnosis of RA because of potential late manifestation of the clinically apparent symptoms of RA. This was done both in cases and matched controls.

8 Sensitivity analysis: investigating the influence of statin use started near the beginning of the patient's electronic record by conducting an analysis where we excluded patients who used statins six months after entrance of the study.

9 Sensitivity analysis: studying the association between statin use and RA in patients without a medical record for cardiovascular disease, diabetes, and hypertension in order to minimise confounding by indication.

10 Sensitivity analysis: investigating the influence of the inclusion of patients with a diagnosis for hepatic diseases, asthma and psoriasis, and antibiotic usage.

11 Sensitivity analysis: excluding subjects who did not have a medical history in the general practice database in order to minimise possible selection bias. 


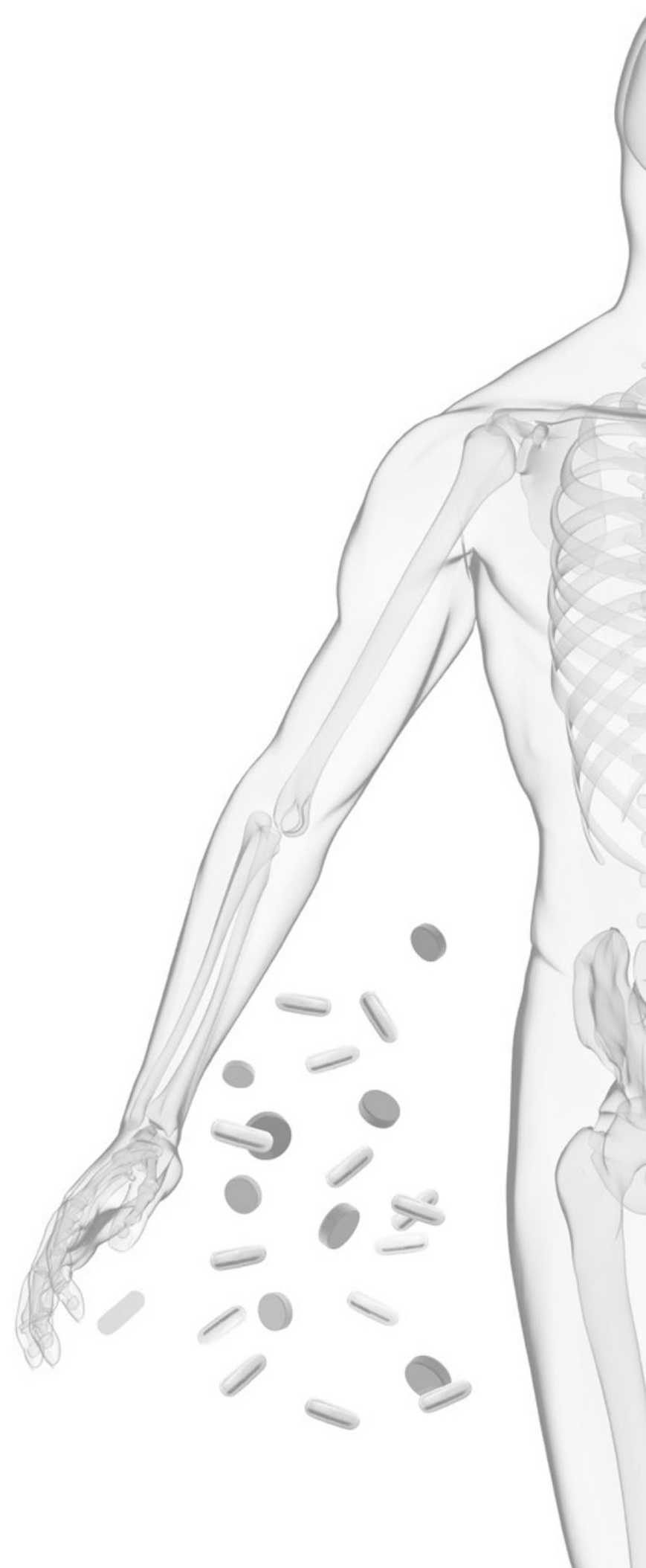




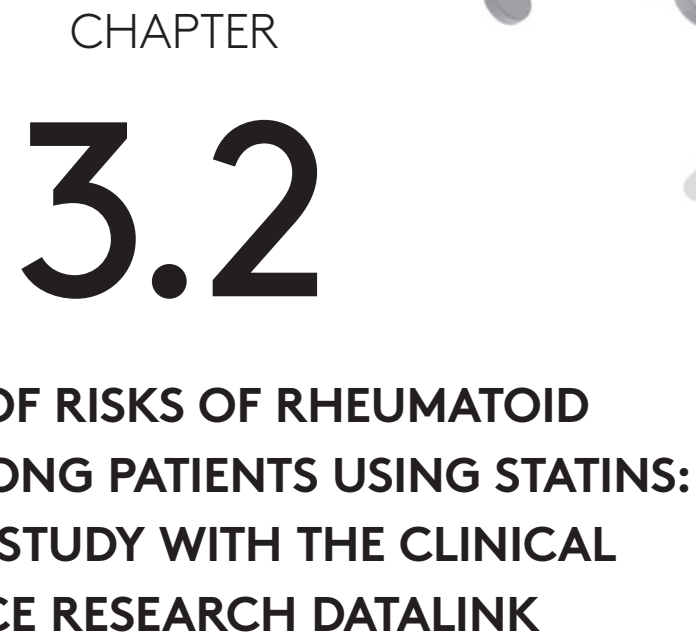

H.J.I. De Jong, J.W. Cohen Tervaert, A. Lalmohamed, F. De Vries, R.J. Vandebriel, H. Van Loveren, O.H. Klungel, T.P. Van Staa 


\section{ABSTRACT}

\section{Objective}

To examine the association between the use of statins and the risk of rheumatoid arthritis (RA), with a special focus on describing the patterns of risks of RA during statin exposure.

\section{Methods}

A retrospective cohort study using the UK Clinical Practice Research Datalink was conducted. All patients aged $\geq 40$ years, who had at least one prescription of statins during the period 1995 to 2009, were selected and matched by age, sex, practice and date of first prescription of statins to controls (non-users). The follow-up period of statin users was divided into periods of current, recent and past exposure, with patients moving between these three exposure categories over time. All patients were followed up for the development of RA. Time-dependent Cox models were used to derive hazard ratios (HRs) of RA, adjusted for disease history and previous drug use.

\section{Results}

The study population included 1023240 patients, of whom 511620 were statin users. No associations were found between RA and current or past users of statins. However, in patients who currently used statins, hazard rates were increased shortly after the first prescription of statins and then gradually decreased to baseline level. The risk of developing RA was increased in patients who recently used statins, as compared to non-users. The risk of RA was highest among women and restart users.

\section{Conclusions}

The risk of RA is substantially increased in the first year after the start of statins and then diminishes to baseline level. The risk of RA was highest among women and restart users. These findings suggest that statins might accelerate disease onset in patients (genetically) susceptible to develop RA. Alternatively, confounding by cardiovascular risk factors and ascertainment or detection bias may have influenced the findings. 


\section{INTRODUCTION}

Statins (3-hydroxy-3-methylglutaryl coenzyme A reductase inhibitors) are widely prescribed drugs to reduce the risk of cardiovascular morbidity and mortality 1 . Besides their cholesterol-lowering activity, statins exert anti-inflammatory and immunomodulatory effects, and may suppress the expression of ongoing autoimmune responses, e.g., rheumatoid arthritis (RA) and multiple sclerosis ${ }^{2-10}$. The immunomodulating effects may, on the other hand, facilitate the development of autoimmunity potentially resulting in autoimmune diseases, such as RA 11-15.

Six other studies have assessed the risk of developing RA or connective tissue disease (CTD) (the majority of the patients were coded with RA) during statin treatment, and have shown conflicting results 14, 16-20. Possible explanations for these conflicting findings may be attributed to considering a lag-time between statin use and incident RA, ${ }^{17,19}$ using different definitions of RA ${ }^{14,17-20}$ or exposure to statins, $14,16-20$ comparing to a control group of non-persistent statin users in stead of non-users, 17 controlling for other confounders, 14, 16-20 shifting the date of incident RA, ${ }^{14,16}$ propensity score matching on baseline characteristics, 20 or conducting separate analyses in patients with or without a medical history of cardiovascular risk factors ${ }^{14,16,17}$. Cardiovascular risk factors, including smoking and hormone replacement therapy have been associated with RA 21-26. Several studies have demonstrated an unfavourable lipid profile before a patient is diagnosed with $R A 21,27$. If statin use is a proxy for hyperlipidaemia then the increased risk of developing RA in our previous study 14 might be explained by hyperlipidaemia rather than by an immunomodulating effect of statins. Otherwise, subclinical RA may have been present before the initiation of statin treatment since it is well-known that autoantibodies and non-specific symptoms may be present long before patients are diagnosed as having RA 28 .

At present, it is unclear to what extent the association between statin use and the risk of developing RA is related to statins or if it is merely an association with hyperlipidaemia. Moreover, none of the previous studies 14, 16-20 studied the pattern of risks of RA with changes in statin exposure. Therefore, we examine the association between the use of statins and the risk of RA, with a special focus on describing the patterns of risks of RA with changes in statin exposure over time and confounding by cardiovascular co-morbidities in a large population-based cohort.

\section{METHODS}

\section{Data source}

Data were derived from the Clinical Practice Research Datalink (CPRD), previously known as the General Practice Research Database, which contains computerised medical records of all patients under the care of 625 general practitioners (GPs) in the 
United Kingdom, representing 8\% of the population. The CPRD has been described in detail elsewhere 29,30 . The database provides detailed information on demographics, diagnoses, prescription details, preventive care provided, specialist referrals, and hospital admissions 30 . Several independent validation studies have shown that the CPRD database has a high level of completeness and validity 31, 32. All research undertaken using data obtained from CPRD is approved by, as appropriate, an ethics committee, a scientific committee and the National Information Governance Board Ethics and Confidentiality Committee.

\section{Study population}

We conducted a matched retrospective cohort study among patients aged 40 years or older, who had at least one prescription of statins at least one year after the start of data collection (period: 1995-2009). The date of the first prescription of statins was defined as the index date. Statin users were matched by age, sex, and practice to a single patient who did not receive a statin prescription (control) any time during follow-up, with the index date of the control being the same as that of the statin user. After using a matched random sampling approach, statin users and non-users who had been diagnosed with RA, or used disease modifying anti-rheumatic drugs (DMARDs) before the index date were excluded. In addition their corresponding non-user or statin user was excluded from the study.

\section{Exposure to statins}

All prescriptions for statins were identified. Each prescription length was calculated by dividing the number of prescribed tablets by the prescribed daily dose. Since statin therapy compliance declines substantially over time 33 , the time of follow-up was divided into periods of current, recent and past exposure to statins, with patients moving between these three exposure categories over time ${ }^{34}$. Current exposure was defined as the time from the date of a prescription until three months after its expected duration of use. The expected duration of statin use was defined as three months. When the consecutive prescription of statins was prescribed within these three months, patients continued to be 'current users'. We divided current users into 'de novo' and 'restart' users. 'De novo' statin users were defined as patients who were not moving between the three periods of statin exposure over time. Current, 'de novo' and 'restart' statin users were also classified according to duration of use ( $\leq 1$ year use; $>1$ year use). Recent exposure was defined as the period of time from three to twelve months after the end date of the most recent prescription, and past exposure was the period of time from twelve months or longer after the end date of the most recent prescription of statins (Figure 1). 


\section{Patient A}

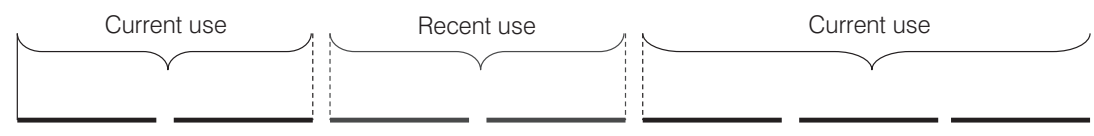

Patient B

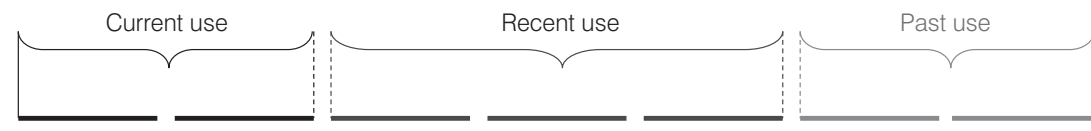

\section{Patient C}

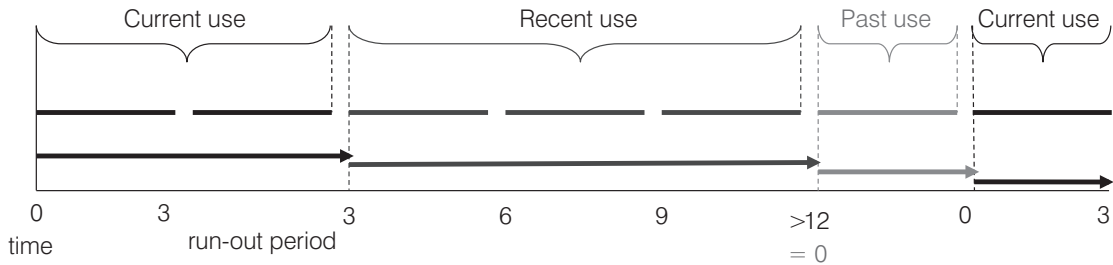

Figure 1 Three examples of time-dependent exposure to statins in patients $\mathrm{A}, \mathrm{B}$ and $\mathrm{C}$.

\section{Clinical outcome}

Each patient was followed from the index date up to either the end date of data collection, or the date when the patient left the general practice, died, or was first diagnosed with RA (identified from the CPRD Read codes) whichever date came first. Patients were considered as having a diagnosis of RA if the first-time diagnosis registered by GPs was verified by at least one prescription of DMARDs during follow-up, adapted from an algorithm proposed by Thomas et al. ${ }^{35}$. Patients who did not meet the algorithm criterion during follow-up were censored at the last date of data collection, or date when they left the practice, or date of death, whichever came first. When RA occurred or when a patient was previously referred to a rheumatologist, the date of the first-time diagnosis of RA or referral to a rheumatologist was defined as the event date.

\section{Potential confounders}

A list of potential confounders before the index date was assembled, which included body mass index (BMI), smoking and alcohol status (a record of currently smoking or drinking, ex-smoker or-drinker, or never smoked or drank before; missing data in 
both variables were treated as a separate category in the analyses), and a history of hypertension, diabetes mellitus, hyperlipidaemia, cardiovascular disease, asthma, inflammatory bowel and thyroid disease, and cancer 23, 36, 37. Patients were classified as diabetes mellitus when they had a diagnosis of diabetes mellitus or when they received anti-diabetic therapy. Hypertension was defined as using anti-hypertensive drugs or a diagnosis of hypertension. Data on the use of non-steroidal anti-inflammatory drugs (NSAIDs), aspirin, proton pump inhibitors (PPIs), antibiotics, hormone replacement therapy, antidepressants, anticonvulsants, anti-psychotics, anti-arrhythmic and other lipid-lowering agents within six months before the index date were considered as potential confounders 38 .

\section{Statistical analysis}

We estimate the hazard ratios (HRs) and 95\% confidence intervals (Cls) for the risk of developing RA among statin users, using a time-dependent Cox proportional hazards model (SAS 9.2. PHREG procedure). First, we assessed the risk of RA between current, recent and past users versus non-users of statins. Second, a descriptive analysis of the pattern of changes in the risk of RA (hazard rates) in current statin users compared to non-users (with past and recent users defined as time $=0$ ) was performed. The risk of RA was plotted against the time since the first prescription of current statin use and visualised using smoothing spline regression ${ }^{39}$, which has been advocated as an alternative to categorical analysis 40 .

Potential confounders were included in the final model if they independently changed the $\beta$ coefficient for statin use by at least $5 \%$. To examine residual confounding due to the omission of confounding variables from the adjustment model 41 , we included all potential confounders in the model.

Because of potential confounding effects due to the presence of cardiovascular diseases or related risk factors, the analysis was stratified according to history of cardiovascular diseases, hypertension and diabetes. According to a NICE clinical guideline lipid modification ${ }^{42}$, statins could have been prescribed to patients with diabetes mellitus, or low socioeconomic status, or a family history of cardiovascular disease or a high-risk ethnicity, regardless of their lipid levels, and therefore; we conducted a subgroup analysis in patients with or without a medical history of hyperlipidaemia. The incidence of RA progressively increases with age, but steadily declines after 65 years ${ }^{43}$, and women are two to three times more affected with RA than men ${ }^{43}$. Therefore, age- and sex-stratified analyses for the evaluation of effect modification were carried out.

\section{Sensitivity analysis}

We evaluated the impact of potential case misclassification by changing the definition of RA into patients with a first-time diagnosis of RA with a referral to a rheumatologist 
or one prescription of DMARDs. Secondly, we defined RA as the first-time diagnosis of RA with a referral to a rheumatologist, or at least one prescription of DMARDs and/ or at least two prescriptions of corticosteroids, a definition we used in a previous study ${ }^{14}$. Thirdly, we identified RA as having another medical record of RA after the first-time diagnosis 44 .

As previously illustrated by two population-based cohort studies ${ }^{17,19}$, it is advisable to take into account a lag time between the onset of RA and the diagnosis. We therefore excluded the first year following the initiation of statin treatment.

Because of potential late manifestation of the clinically apparent symptoms of RA, we considered the date of RA exactly one year before the first-time diagnosis of RA as suggested by Jick et al.16.

Lastly, by limiting bias due to 'peeking into the future', we considered patients as having RA when they were treated with at least one prescription of a DMARD and referred to a rheumatologist within a time span of two years after their first-time diagnosis.

\section{RESULTS}

After excluding 40320 patients who were younger than 40 years, 31460 patients with a medical history of RA and 12968 patients with prescriptions of DMARDs before the index date, 511620 statin users and 511620 non-users were enrolled in the study (Figure 2). Due to matching, statin users and non-users had similar distributions of age (statin users: mean age, 63.0 years and non-users: mean age, 62.8 years) and sex (statin users and non-users: $48 \%$ women). Statin users were more often diagnosed with cardiovascular diseases, hyperlipidaemia, hypertension, diabetes and cerebrovascular events. Remarkably, smoking was not different between statin users and non-users. However, ex-smoking was higher in statin users than non-users. Based on the high prevalence of cardiovascular risk factors and/or disease in statin users, aspirin, anti-hypertensive and anti-diabetic agents were more prescribed in statin users compared to non-users. Furthermore, statin users were more likely to have used NSAIDs, PPIs, antibiotics and antidepressants than non-users. The baseline characteristics of the statin users and non-users are presented in Table 1.

Current users did not show a risk of developing RA ( $H R_{\text {adj }}, 1.08 ; 95 \% \mathrm{Cl}, 0.94$ to 1.24) (Table 2). However, current statin users who continued the therapy for $\leq 1$ year had a 1.3-fold increased risk of developing RA, adjusted (adj) HR, 1.29; 95\% Cl, 1.08 to 1.55. The HR $\mathrm{Hadj}_{\mathrm{aj}}$ for 'de novo' users and 'restart' users were 1.01 ; $(95 \% \mathrm{Cl}, 0.87$ to 1.17$)$ and 1.18; (95\% Cl, 1.01 to 1.38), respectively. Restart users who continued the therapy for $\leq 1$ year had also a 1.3-fold increased risk of $\mathrm{RA}\left(\mathrm{HR}_{\mathrm{adj}}, 1.25 ; 95 \% \mathrm{Cl}, 1.03\right.$ to 1.53$)$ 


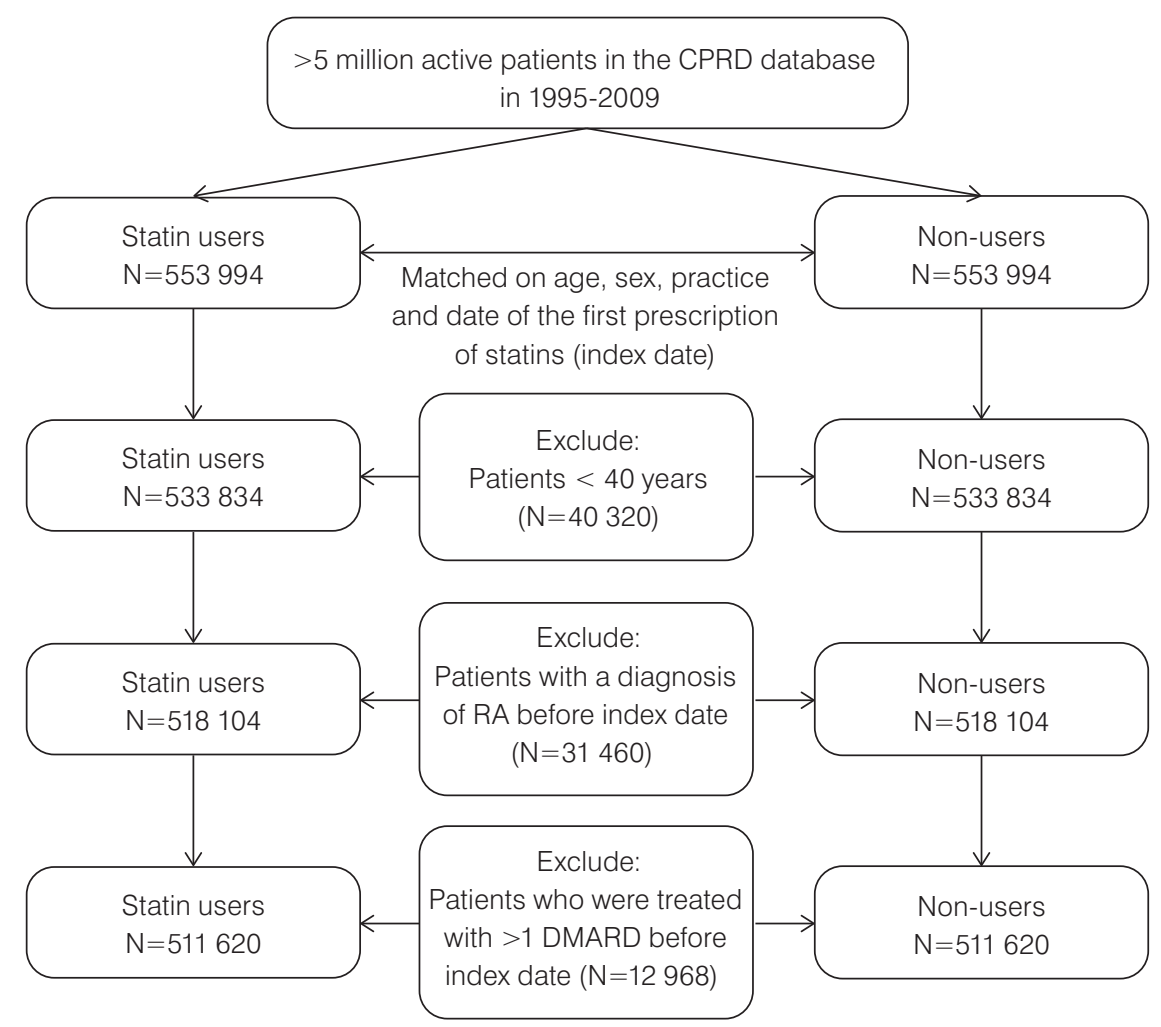

Figure 2 Flow chart Study population.

CPRD, Clinical Practice Research Datalink; RA, rheumatoid arthritis; DMARD, disease modifying antirheumatic drug

whereas 'de novo' users showed no risk of developing $\mathrm{RA}\left(\mathrm{HR}_{\mathrm{adj}}, 1.17 ; 95 \% \mathrm{Cl}, 0.95\right.$ to 1.44). Risk of RA was 1.4-fold increased with recent statin use, as compared to non-users ( $\mathrm{HR}_{\mathrm{adj}}, 1.41 ; 95 \% \mathrm{Cl}, 1.12$ to 1.79). No association was found between past statin users and incident RA.

Figure 3 shows that the risk of RA was substantially increased in the first year after the first prescription of statins compared to non-users. The $\mathrm{HR}_{\text {adj }}$ was $2.96(95 \% \mathrm{Cl}$, 2.51 - 3.49). After one year of statin exposure, the risk of RA declined to baseline level (> 1 year: $\mathrm{HR}_{\mathrm{adj}}, 0.92 ; 95 \% \mathrm{Cl}, 0.69-1.23$ ). Similar results were obtained when we included all potential confounders in our model. 
Table 1 Baseline characteristics of statin users and non-users

\begin{tabular}{|c|c|c|}
\hline Baseline characteristics & $\begin{array}{l}\text { Statin users } \\
(n=511620)\end{array}$ & $\begin{array}{c}\text { Non-users } \\
(\mathrm{n}=511620)\end{array}$ \\
\hline \multicolumn{3}{|l|}{ Duration of follow-up (years) } \\
\hline Mean (SD) & $3.4(2.5)$ & $3.1(2.6)$ \\
\hline \multicolumn{3}{|l|}{ Sex, n (\%) } \\
\hline Women & $244870(47.9)$ & $244870(47.9)$ \\
\hline \multicolumn{3}{|l|}{ Age (years) } \\
\hline Mean (SD) & $63.0(12.1)$ & $62.8(12.5)$ \\
\hline \multicolumn{3}{|l|}{ Age by category, years (\%) } \\
\hline $40-49$ & $69713(13.6)$ & $74284(14.5)$ \\
\hline $50-59$ & $147001(28.7)$ & $156841(30.7)$ \\
\hline $60-79$ & 237736 (46.5) & $216749(42.4)$ \\
\hline $80+$ & $57170(11.2)$ & $63746(12.4)$ \\
\hline \multicolumn{3}{|l|}{ BMI (kg/m²) } \\
\hline Mean (SD) & $26.9(8.4)$ & $21.0(11.6)$ \\
\hline \multicolumn{3}{|l|}{ Smoking status, n (\%) } \\
\hline Non-smoker & $213102(41.7)$ & $230927(45.1)$ \\
\hline Ex-smoker & 161885 (31.6) & 109645 (21.5) \\
\hline Smoker & 114085 (22.3) & $99340(19.4)$ \\
\hline Unknown smoking status & $22548(4.4)$ & $71708(14.0)$ \\
\hline \multicolumn{3}{|l|}{ Drinking status, n (\%) } \\
\hline Non-drinker & $63872(12.5)$ & $53309(10.4)$ \\
\hline Ex-drinker & $32104(6.3)$ & $20384(4.0)$ \\
\hline Drinker & 352827 (68.9) & $317067(62.0)$ \\
\hline Unknown drinking status & 62817 (12.3) & $120860(23.6)$ \\
\hline \multicolumn{3}{|l|}{ Drug use within previous 6 months, n (\%) } \\
\hline Antihypertensive agents & $317494(62.1)$ & $121220(23.7)$ \\
\hline Fibrates & $8436(1.6)$ & $881(0.2)$ \\
\hline Ezetimibe & $1943(0.4)$ & $130(0.03)$ \\
\hline Anti-diabetic agents & $120353(23.5)$ & $18200(3.6)$ \\
\hline Anti-arrhythmic agents & $20207(3.9)$ & $11051(2.2)$ \\
\hline Aspirin & $142209(27.8)$ & $36003(7.0)$ \\
\hline NSAIDs & $197750(38.7)$ & $86106(16.8)$ \\
\hline Proton pump inhibitors & $82939(16.2)$ & $46820(9.2)$ \\
\hline Hormone replacement therapy or oral contraceptives & $21219(4.1)$ & $20598(4.0)$ \\
\hline Oral corticosteroids & $16815(3.3)$ & $14684(2.9)$ \\
\hline
\end{tabular}


Table 1 Continued

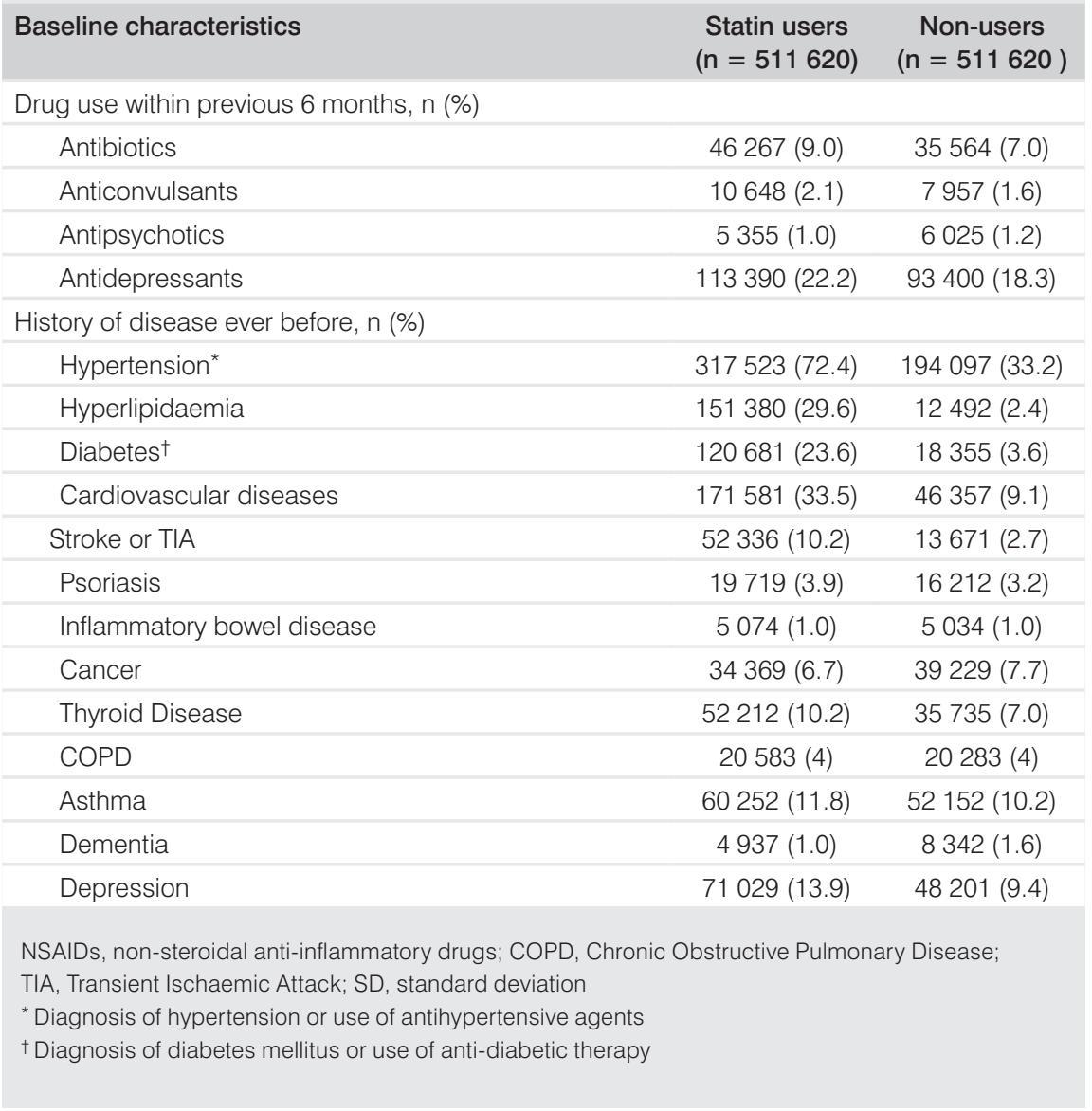

In Table 3, we present several potential factors that may have influenced the risk of developing RA after statin exposure. We found an effect modification by sex. In women, current and recent statin use was associated with incident RA, whereas no associations were found in men. The effect of developing RA in women who currently used statins was only observed in the first year of current statin use. We only observed an increased risk of developing RA in patients without a medical record of hyperlipidaemia, hypertension and diabetes who currently used statins for less than one year or who were recent users. No other effect modifiers for the association between current, recent and past statin exposure and incident RA were found. 
Table 2 Risk of rheumatoid arthritis in statin users compared to non-users

\begin{tabular}{|c|c|c|c|c|}
\hline & $\begin{array}{l}\mathrm{RA} \\
(\mathrm{n})\end{array}$ & $\mathrm{IR}^{*}$ & $\begin{array}{c}\text { age- and sex-adjusted } \\
\text { HR }(95 \% \mathrm{Cl})\end{array}$ & $\begin{array}{l}\text { fully adjusted } \\
\mathrm{HR}(95 \% \mathrm{Cl}){ }^{\dagger}\end{array}$ \\
\hline No statin use & 579 & 3.7 & 1.00 & 1.00 \\
\hline Past statin use & 105 & 4.9 & $1.33(1.08-1.64)$ & $1.20(0.96-1.49)$ \\
\hline Recent statin use & 101 & 5.6 & $1.60(1.27-2.02)$ & $1.41(1.12-1.79)$ \\
\hline Current statin use & 837 & 4.3 & $1.24(1.10-1.38)$ & $1.08(0.94-1.24)$ \\
\hline$\leq 1$ year & 386 & 11.5 & $1.47(1.25-1.73)$ & $1.29(1.08-1.55)$ \\
\hline$>1$ year & 451 & 2.8 & $1.15(1.02-1.31)$ & $1.00(0.86-1.16)$ \\
\hline 'de novo' statin use & 464 & 4.1 & $1.16(1.02-1.31)$ & $1.01(0.87-1.17)$ \\
\hline$\leq 1$ year & 172 & 15.8 & $1.33(1.10-1.61)$ & $1.17(0.95-1.44)$ \\
\hline$>1$ year & 292 & 2.9 & $1.09(0.95-1.26)$ & $0.94(0.80-1.11)$ \\
\hline 'restart' statin use & 373 & 4.7 & $1.34(1.17-1.54)$ & $1.18(1.01-1.38)$ \\
\hline$\leq 1$ year & 214 & 9.4 & $1.42(1.19-1.70)$ & $1.25(1.03-1.53)$ \\
\hline$>1$ year & 159 & 2.8 & $1.27(1.06-1.52)$ & $1.10(0.91-1.34)$ \\
\hline
\end{tabular}

RA, rheumatoid arthritis; IR, incidence rate (per 10000 person-years); HR, hazard ratio; $\mathrm{Cl}$, confidence interval * Incidence rate is calculated for each recency of statin use by dividing the number of events by the person time within each given recency of use.

† Adjusted for age, sex, smoking, cardiovascular diseases, hyperlipidaemia, hypertension, diabetes and use of non-steroid anti-inflammatory drugs.

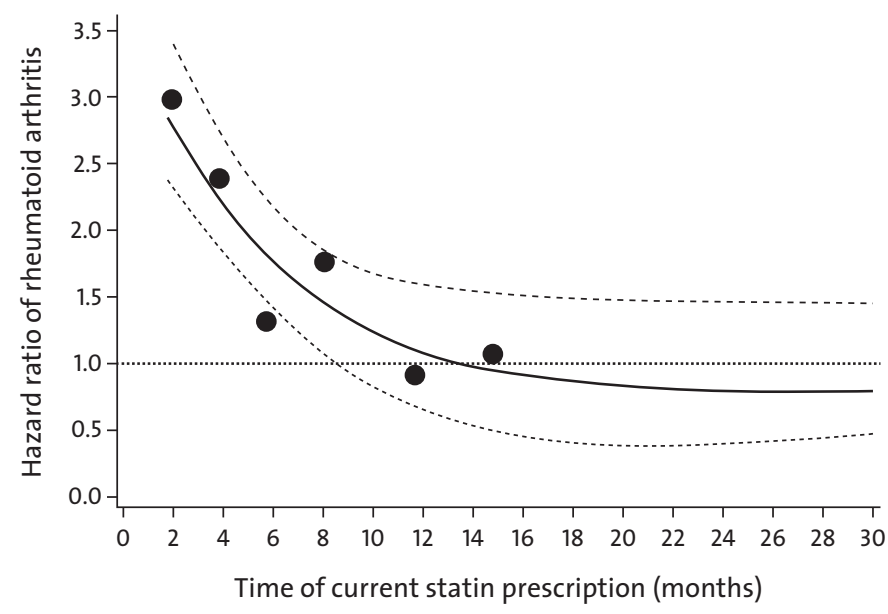

Figure 3 Risk of rheumatoid arthritis in current statin users versus non-users, by time since the first statin prescription.

Solid bold line and circles: adjusted hazard ratios. Solid lines: 95\% confidence bands. 
Table 3 Confounding and modifying effects of rheumatoid arthritis risk in statin users vs. non-users
RA
$\mathrm{IR}^{*}$
Past statin use

(n)

\begin{tabular}{rrrr} 
By age, $y$ & & & \\
\hline $40-50$ & 131 & 2.6 & $2.17(0.91-4.32)$ \\
\hline $51-60$ & 475 & 4.1 & $1.13(0.76-1.70)$ \\
\hline $61-80$ & 938 & 5.1 & $1.09(0.81-1.48)$ \\
$>80$ & 78 & 2.0 & $1.23(0.51-2.94)$
\end{tabular}

By sex

$\begin{array}{lrrr}\text { Women } & 1021 & 5.4 & 1.21(0.92-1.58) \\ \text { Men } & 601 & 3.0 & 1.21(0.83-1.74)\end{array}$

By any previous history of disease

\begin{tabular}{lrrr}
\hline No previous cardiovascular disease & 1138 & 3.9 & $1.26(0.99-1.62)$ \\
\hline Previous cardiovascular disease & 484 & 4.8 & $0.96(0.59-1.57)$ \\
\hline No previous cardiovascular risk factor ${ }^{\ddagger}$ & 649 & 4.0 & $1.20(0.83-1.75)$ \\
\hline Previous cardiovascular risk factor & 973 & 4.3 & $1.06(0.80-1.41)$ \\
\hline No previous hyperlipidaemia & 1254 & 4.1 & $1.13(0.87-1.47)$ \\
\hline Previous hyperlipidaemia & 368 & 4.5 & $1.71(0.82-3.54)$ \\
\hline No previous hypertension & 857 & 4.1 & $1.27(0.94-1.72)$ \\
\hline Previous hypertension & 765 & 4.3 & $0.99(0.72-1.37)$ \\
\hline No previous diabetes & 1394 & 4.2 & $1.19(0.94-1.50)$ \\
\hline Previous diabetes & 228 & 4.1 & $1.11(0.52-2.34)$
\end{tabular}

RA, rheumatoid arthritis; IR, incidence rate (per 10000 person-years); $\mathrm{HR}$, hazard ratio; $\mathrm{Cl}$, confidence interval * Incidence rate is calculated for each recency of statin use by dividing the number of events by the person time within each given recency of use.

${ }^{\dagger}$ Adjusted for confounders as shown in table 2.

‡Cardiovascular risk factor included previous hyperlipidaemia, hypertension and diabetes.

Performing different sensitivity analyses did alter our findings slightly. However, when we depicted the five patterns of changes in the risk of RA during current statin use, no differences in the patterns of the five sensitivity analyses were observed. Only, the sensitivity analysis where we excluded the first year after initiation of statin treatment, the pattern of risks of RA during statin use was slightly attenuated. No difference in the risk of developing RA was observed between current users who continued the 
adjusted HR $(95 \% \mathrm{Cl})^{\dagger}$

\begin{tabular}{|llcc}
\hline Recent statin use & Current statin use & $\begin{array}{c}\text { Current statin use } \\
\leq 1 \text { year }\end{array}$ & $\begin{array}{c}\text { Current statin use } \\
\text { > year }\end{array}$ \\
\hline $1.60(0.66-3.86)$ & $1.31(0.76-2.26)$ & $1.07(0.54-2.15)$ & $1.41(0.80-2.50)$ \\
\hline $1.63(1.06-2.50)$ & $0.96(0.73-1.25)$ & $1.31(0.93-1.84)$ & $0.82(0.61-1.10)$ \\
\hline $1.21(0.87-1.69)$ & $1.02(0.85-1.22)$ & $1.23(0.97-1.56)$ & $0.95(0.78-1.15)$ \\
\hline $1.51(0.59-3.88)$ & $0.95(0.53-1.71)$ & $1.08(0.52-2.25)$ & $0.87(0.45-1.69)$ \\
\hline & & & \\
\hline $1.71(1.27-2.31)$ & $1.19(1.00-1.42)$ & $1.51(1.20-1.91)$ & $1.08(0.90-1.31)$ \\
\hline $1.00(0.66-1.52)$ & $0.90(0.71-1.14)$ & $1.00(0.75-1.33)$ & $0.86(0.67-1.10)$ \\
\hline $1.37(1.02-1.84)$ & $1.02(0.86-1.20)$ & $1.21(0.97-1.50)$ & $0.93(0.77-1.12)$ \\
\hline $1.33(0.83-2.12)$ & $1.06(0.76-1.47)$ & $1.28(0.87-1.90)$ & $0.98(0.70-1.39)$ \\
\hline $1.55(1.02-2.35)$ & $1.06(0.85-1.33)$ & $1.40(1.05-1.88)$ & $0.86(0.65-1.14)$ \\
\hline $1.20(0.88-1.62)$ & $0.95(0.78-1.15)$ & $1.10(0.86-1.39)$ & $0.90(0.74-1.10)$ \\
\hline $1.62(1.23-2.12)$ & $1.07(0.92-1.25)$ & $1.23(1.00-1.50)$ & $1.00(0.85-1.18)$ \\
\hline $1.41(0.65-3.07)$ & $1.47(0.75-2.88)$ & $1.92(0.94-3.92)$ & $1.34(0.68-2.64)$ \\
\hline $1.31(0.92-1.87)$ & $1.14(0.94-1.39)$ & $1.53(1.19-1.96)$ & $0.96(0.77-1.20)$ \\
\hline $1.30(0.92-1.82)$ & $0.89(0.72-1.10)$ & $0.96(0.74-1.26)$ & $0.86(0.69-1.08)$ \\
\hline $1.42(1.09-1.85)$ & $1.09(0.94-1.26)$ & $1.34(1.10-1.62)$ & $0.99(0.84-1.16)$ \\
\hline $1.14(0.55-2.38)$ & $0.90(0.50-1.60)$ & $0.92(0.49-1.75)$ & $0.88(0.49-1.60)$ \\
\hline
\end{tabular}

therapy for $\leq 1$ year and $>1$ year. The results of the six sensitivity analyses are presented online (Appendix 1). 


\section{DISCUSSION}

This study demonstrated a 1.3-fold increased risk of developing RA during the first year of statin use. The risk of developing RA was increased shortly after the first prescription of statins and then gradually decreased to baseline level. The risk of RA was highest among women and restart users. In recent users, statin use was associated with incident RA whereas in past users no such effect was found.

Two population-based cohort studies showed no association between statin use and the risk of developing RA ${ }^{18}, 19$. In a nested case-control study of 313 incident RA patients and 1252 matched controls, no association between current statin use and incident RA was found ${ }^{16}$. The same study showed, however, in a subsample of only patients with hyperlipidaemia a decreased risk of developing RA in current users ${ }^{16}$. We found no association between statin use and incident RA in patients with hyperlipidaemia. However, these conflicting results may be explained by differences in controlling for confounders. In addition to the matching factors, we adjusted for cardiovascular disease, hypertension, diabetes and smoking whereas Jick et al. adjusted only for smoking 16 .

In a population-based cohort study of 211627 new statin users, statin use was associated with a reduction in the risk of developing RA. This effect was only present for those who used statins for more than one year 17. In contrast, we found no association between statin use and incident RA in current users who continued statin therapy for more than one year. In a propensity score matched cohort study of 6956 pairs of statin users and non-users, statin use was associated with a lower risk of CTD20. The possible protective effect of statins was not observed in our study. The discrepancies may be partially explained by the difference in defining statin exposure and RA. We classified statin exposure by the recency of use, and modelled it as a time-dependent variable. Chodick et al. defined statin exposure as the mean proportion of follow-up days covered with statins ${ }^{17}$, whereas Schmidt et al. defined statin use as receiving at least a 90 -day supply at baseline 20 . Our definition of RA may have been more specific than the one used by the other two studies 17, 20. We verified patients' electronic records with at least one prescription of DMARDs after the diagnosis of RA. In the study by Chodick et al., patients were included when they had a diagnostic code for RA with or without the use of DMARDs ${ }^{17}$, whereas Schmidt et al. included patients with CTD, including RA 20 . By using a more specific definition of RA, we may have attenuated the association between statin use and incident RA.

In our previous study ${ }^{14}$, we found an increased risk of developing RA within six months of statin use, which is in line with the results of the present study. In this study, we found that the risk of developing RA disappeared after one year. Statins may accelerate the onset of RA in patients susceptible to develop RA as previously 
demonstrated by an animal model of arthritis 15 . In line with this hypothesis, the majority of cases of statin-associated lupus-like syndrome developed this syndrome within one year after starting statin therapy 11. However, in patients not prone to develop RA, statins are probably safe and well tolerated, even after prolonged use. This could also explain the difference in the risk of developing RA in 'de novo' and 'restart' users. It may be that 'de novo' users were patients who can tolerate statins whereas 'restart' users were patients who were less likely to be fully adherent to statins because of potential discomfort or side effects. Another explanation could be our observed effect modification by sex: men were not at risk whereas women were at high risk for developing RA. It has already been shown before, that women are more prone to developing RA43, 45 .

RA may have been present and not well documented before the start of statins. In both our studies, we defined the onset date of RA by the first record or specialist referral. Unfortunately, the onset date of symptoms is unknown in our studies. One study reported a median time between onset of symptoms to diagnosis of RA of approximately 36 weeks (range: 4 weeks to $>10$ years) ${ }^{46}$. As two population-based studies considered a lag-time of one year between statin use and incident RA 17, 19, we performed an analysis where we excluded the first year following the initiation of statin treatment. We found no increased risk of RA in the first year after the initiation of statin treatment. However, the descriptive analysis showed a similar pattern of risks of RA during statin use but was slightly attenuated.

Importantly, cardiovascular risk factors (e.g. hyperlipidaemia) may have influenced the association between statin use and incident RA. Several studies have demonstrated an unfavourable lipid profile in patients with RA 21, 27 Hyperlipidaemia may induce leukocyte activation and possibly complement activation, 47-49 which may result in an earlier diagnosis of RA in patients prone to develop RA. When we conducted subgroup analyses in patients with and without these recorded cardiovascular risk factors, no differences in the risk of developing RA between these two groups were observed.

Strengths of this study include its large sample size, representativeness of the population, completeness of follow-up and information on matched controls, and detailed information on confounders, such as smoking status was available 25,36 . We had the opportunity to use routinely recorded medical and prescription data from GPs. Consequently, recall bias was minimised.

Some drawbacks of our study should be considered. Firstly, the information about statin exposure was based on prescription data rather than on actual drug use, which could have resulted in an overestimation of statin use.

Secondly, we may have used a relatively sensitive, but non-specific, diagnosis of RA as we had limited information on rheumatoid factor and anti-cyclic citrullinated 
peptide antibodies 28. In this study, we have used the diagnostic algorithm as postulated by Thomas et al. 35 . The proposed diagnostic algorithm resulted in a diagnostic specificity of $96 \% 35$. By applying this diagnostic algorithm, we believe we have used a relatively accurate diagnosis of RA. Furthermore, we performed a range of sensitivity analyses regarding the definition of RA. All these analyses consistently showed similar results.

Thirdly, no data on dietary intake, physical activity, and limited data were available on other examinations such as lipid, blood pressure and glucose levels, and inflammatory markers (e.g. C-reactive protein), which may be important confounders. Especially, in the subgroup analyses based on the cardiovascular risk factors, lack of clinical data may have affected our results. It is likely that we have included patients with high lipid, glucose or high blood pressure levels in the group of patients without a medical history of hyperlipidaemia, hypertension or diabetes.

Fourthly, ascertainment bias may have been present in our study because patients starting statin therapy may have a careful follow-up, including visits to the GP and blood tests, thereby increasing the likelihood of detecting more abnormalities (e.g. RA) than non-users 18,50 .

Fifth, the increased risk of RA in the first year after the initiation of statin therapy may be explained by detection bias, as some patients initiating statin therapy may experience myalgia or other muscle-related adverse effects; 51 they may tend to visit their GP more often, be more likely to be referred to a rheumatologist and may have been more carefully examined, and therefore; these patients may be more likely to be diagnosed with RA.

To our knowledge, this is the first study evaluating risks of RA in current statin users over time. In patients who use statins, the risk of RA is substantially increased in the first year after initiation of statins and then diminishes to baseline. The risk of RA was highest among women and restart users. These findings suggest that statins can accelerate disease onset in patients (genetically) susceptible to develop RA. The alternative explanation for this increased risk of RA shortly after starting statins is ascertainment or detection bias with increased diagnostic monitoring around the time of initiation of statin therapy. Although more research is needed, this study supports our previous finding, showing an increased risk of developing RA shortly after starting statin treatment.

\section{What is already known on this topic}

Six other studies have assessed the risk of developing RA or connective tissue diseases during statin treatment, and have shown conflicting results.

It is not clear to what extent the association between statin use and the risk of developing RA is related to statins or to co-occurring hyperlipidaemia. 
None of the previous six studies described the pattern of risks of RA (hazard rates) with changes in statin exposure over time.

\section{What this study adds}

The risk of developing RA is substantially increased in the first year after the start of statin therapy; no increased risk is found during follow-up of statin treatment.

The risk of RA was highest among women and restart users.

This suggests that statins can accelerate disease onset in patients (genetically) susceptible to develop RA, as has been found by us in an animal model of arthritis. An alternative explanation for our findings is ascertainment or detection bias with increased diagnostic monitoring around the time of initiation of statin therapy.

\section{Acknowledgements}

The authors thank MSc A.M. Gallagher for providing the data for the present study and Dr S.L. Thomas for sharing the Read codes for RA. 


\section{REFERENCES}

1 Baigent C, Keech A, Kearney PM, et al. Efficacy and safety of cholesterol-lowering treatment: prospective meta-analysis of data from 90,056 participants in 14 randomised trials of statins. Lancet 2005; 366(9493): 1267-78.

2 Ridker PM, Danielson E, Fonseca FA, et al. Reduction in C-reactive protein and LDL cholesterol and cardiovascular event rates after initiation of rosuvastatin: a prospective study of the JUPITER trial. Lancet 2009; 373(9670): 1175-82.

3 McCarey DW, Mclnnes IB, Madhok R, et al. Trial of Atorvastatin in Rheumatoid Arthritis (TARA): double-blind, randomised placebo-controlled trial. Lancet 2004; 363(9426): 2015-21.

4 Arnaud C, Braunersreuther V, Mach F. Toward immunomodulatory and anti-inflammatory properties of statins. Trends Cardiovasc Med 2005; 15(6): 202-6.

5 Kanda H, Yokota K, Kohno C, et al. Effects of low-dosage simvastatin on rheumatoid arthritis through reduction of Th1/Th2 and CD4/CD8 ratios. Mod Rheumatol 2007; 17(5): 364-8.

6 Funk JL, Chen J, Downey KJ, Clark RA. Bone protective effect of simvastatin in experimental arthritis. J Rheumatol 2008; 35(6): 1083-91.

7 Youssef S, Stüve O, Patarroyo JC, et al. The HMG-CoA reductase inhibitor, atorvastatin, promotes a Th2 bias and reverses paralysis in central nervous system autoimmune disease. Nature 2002; 420(6911): 78-84.

8 Okamoto H, Koizumi K, Kamitsuji S, et al. Beneficial action of statins in patients with rheumatoid arthritis in a large observational cohort. J Rheumatol 2007; 34(5): 964-8.

9 Van Doornum S, McColl G, Wicks IP. Atorvastatin reduces arterial stiffness in patients with rheumatoid arthritis. Ann Rheum Dis 2004; 63(12): 1571-5.

10 Hermann F, Forster A, Chenevard R, et al. Simvastatin improves endothelial function in patients with rheumatoid arthritis. J Am Coll Cardiol 2005; 45(3): 461-4.

11 Noël B. Lupus erythematosus and other autoimmune diseases related to statin therapy: a systematic review. J Eur Acad Dermatol Venereol 2007; 21(1): 17-24.

12 De Jong HJ, Tervaert JW, Saldi SR, et al. Association between statin use and lupus-like syndrome using spontaneous reports. Semin Arthritis Rheum 2011; 41(3): 373-81.

13 De Jong HJ, Saldi SR, Klungel OH, et al. Statin-Associated Polymyalgia Rheumatica. An Analysis Using WHO Global Individual Case Safety Database: A Case/Non-Case Approach. PLoS One 2012; 7(7): e41289.

14 De Jong $\mathrm{HJ}$, Klungel $\mathrm{OH}$, Van Dijk L, et al. Use of statins is associated with an increased risk of rheumatoid arthritis. Ann Rheum Dis 2012; 71(5): 648-54.

15 Vandebriel RJ, De Jong HJ, Gremmer ER, et al. Statins accelerate the onset of collagen type II-induced arthritis in mice. Arthritis Res Ther 2012; 14(2): R90.

16 Jick SS, Choi H, Li L, Mclnnes IB, Sattar N. Hyperlipidaemia, statin use and the risk of developing rheumatoid arthritis. Ann Rheum Dis 2009; 68(4): 546-51.

17 Chodick G, Amital H, Shalem Y, Kokia E, Heymann AD, Porath A, Shalev V. Persistence with statins and onset of rheumatoid arthritis: a population-based cohort study. PLoS Med 2010; 7(9): e1000336.

18 Hippisley-Cox J, Coupland C. Unintended effects of statins in men and women in England and Wales: population based cohort study using the QResearch database. BMJ 2010; 340: c2197.

19 Smeeth L, Douglas I, Hall AJ, Hubbard R, Evans S. Effect of statins on a wide range of health outcomes: a cohort study validated by comparison with randomized trials. Br J Clin Pharmacol 2009; 67(1): 99-109.

20 Schmidt T, Battafarano DF, Mortensen EM, Frei CR, Mansi I. Frequency of development of connective tissue disease in statin-users versus non-users. Am J Cardiol 2013; 112(6): 883-8.

21 Van Halm VP, Nielen MM, Nurmohamed MT, et al. Lipids and inflammation: serial measurements of the lipid profile of blood donors who later developed rheumatoid arthritis. Ann Rheum Dis 2007; 66(2): 184-8.

22 Solomon $\mathrm{DH}, \mathrm{Kremer} \mathrm{J}$, Curtis JR, et al. Explaining the cardiovascular risk associated with rheumatoid arthritis: traditional risk factors versus markers of rheumatoid arthritis severity. Ann Rheum Dis 2010; 69(11): 1920-5. 
23 Nurmohamed MT. Cardiovascular risk in rheumatoid arthritis. Autoimmun Rev 2009; 8(8): 663-7.

24 Libby P. Role of inflammation in atherosclerosis associated with rheumatoid arthritis. Am J Med 2008; 121(10 Suppl 1): S21-31.

25 Uhlig T, Hagen KB, Kvien TK. Current tobacco smoking, formal education, and the risk of rheumatoid arthritis. J Rheumatol 1999; 26(1): 47-54.

26 Vandenbroucke JP. Oral contraceptives and rheumatoid arthritis. Lancet 1983; 2(8343): 228-9.

27 Steiner G, Urowitz MB. Lipid profiles in patients with rheumatoid arthritis: mechanisms and the impact of treatment. Semin Arthritis Rheum 2009; 38(5): 372-81.

28 Nielen MM, Van Schaardenburg D, Reesink HW, et al. Simultaneous development of acute phase response and autoantibodies in preclinical rheumatoid arthritis. Ann Rheum Dis 2006; 65(4): 535-7.

29 García Rodríguez LA, Pérez Gutthann S. Use of the UK General Practice Research Database for pharmacoepidemiology. Br J Clin Pharmacol 1998; 45(5): 419-25.

30 Walley T, Mantgani A. The UK General Practice Research Database. Lancet 1997; 350(9084): 1097-9.

31 Jick $H$, Jick SS, Derby LE. Validation of information recorded on general practitioner based computerised data resource in the United Kingdom. BMJ 1991; 302(6779): 766-8.

32 Van Staa TP, Abenhaim L, Cooper C, Zhang B, Leufkens HG. The use of a large pharmacoepidemiological database to study exposure to oral corticosteroids and risk of fractures: validation of study population and results. Pharmacoepidemiol Drug Saf 2000; 9(5): 359-66.

33 Benner JS, Glynn RJ, Mogun H, Neumann PJ, Weinstein MC, Avorn J. Long-term persistence in use of statin therapy in elderly patients. JAMA 2002; 288(4): 455-61.

34 Gallagher AM, Smeeth L, Seabroke S, Leufkens HG, van Staa TP. Risk of death and cardiovascular outcomes with thiazolidinediones: a study with the general practice research database and secondary care data. PLoS One 2011; 6(12): e28157.

35 Thomas SL, Edwards CJ, Smeeth L, Cooper C, Hall AJ. How accurate are diagnoses for rheumatoid arthritis and juvenile idiopathic arthritis in the general practice research database? Arthritis Rheum 2008; 59(9): 1314-21

36 Hazes JM, Dijkmans BA, Vandenbroucke JP, De Vries RR, Cats A. Lifestyle and the risk of rheumatoid arthritis: cigarette smoking and alcohol consumption. Ann Rheum Dis 1990; 49(12): 980-2.

37 Pedersen M, Jacobsen S, Klarlund M, Pedersen BV, Wiik A, Wohlfahrt J, Frisch M. Environmental risk factors differ between rheumatoid arthritis with and without auto-antibodies against cyclic citrullinated peptides. Arthritis Res Ther 2006; 8(4): R133.

38 Olsen N. Drug-induced autoimmunity. Best Pract Res Clin Rheumato 2004; 18(5): 677-88.

39 Ramlau-Hansen $\mathrm{H}$. Smoothing counting process intensities by means of kernel functional. Ann Stastist 1983; 11: 453-66.

40 Greenland S. Dose-response and trend analysis in epidemiology: alternatives to categorical analysis. Epidemiology 1995; 6(4): 356-65.

41 Groenwold $\mathrm{RH}$, Klungel OH, Grobbee DE, Hoes AW. Selection of confounding variables should not be based on observed associations with exposure. Eur J Epidemiol 2011; 26(8): 589-93.

42 National Collaborating Centre for Primary Care. Lipid modification: Cardiovascular risk assessment and the modification of blood lipids for the primary and secondary prevention of cardiovascular disease. NICE clinical guideline 67. London, UK: National Institute for Health and Clinical Excellence, 2008 (reissued 2010).

43 Myasoedova E, Crowson CS, Kremers HM, Therneau TM, Gabriel SE. Is the incidence of rheumatoid arthritis rising?: results from Olmsted County, Minnesota, 1955-2007. Arthritis Rheum 2010; 62(6): 1576-82.

44 Kim SY, Servi A, Polinski JM, Mogun H, Weinblatt ME, Katz JN, Solomon DH. Validation of rheumatoid arthritis diagnoses in health care utilization data. Arthritis Res Ther 2011; 13(1): R32.

45 Alamanos Y, Drosos AA. Epidemiology of adult rheumatoid arthritis. Autoimmun Rev 2005; 4(3): 130-6.

46 Chan KW, Felson DT, Yood RA, Walker AM. The lag time between onset of symptoms and diagnosis of rheumatoid arthritis. Arthritis Rheum 1994; 37(6): 814-20.

47 Alipour A, Van Oostrom AJ, Izraeljan A, Verseyden C, Collins JM, Frayn KN, et al. Leukocyte activation by triglyceride-rich lipoproteins. Arterioscler Thromb Vasc Biol 2008; 28(4): 792-7. 
48. Van Oostrom AJ, Rabelink TJ, Verseyden C, Sijmonsma TP, Plokker HW, De Jaegere PP, et al. Activation of leukocytes by postprandial lipemia in healthy volunteers. Arteriosclerosis 2004; 177(1): 175-82.

49. Meijssen S, Van Dijk H, Verseyden C, Erkelens DW, Cabezas MC. Delayed and exaggerated postprandial complement component 3 response in familial combined hyperlipidemia. Arterioscler Thromb Vasc Biol 2002; 22(5): 811-6

50 Mansi I, Mortensen E. The controversy of a wider statin utilization: why? Expert Opin Drug Saf 2013; 12(3): 327-37.

51 Sathasivam S, Lecky B. Statin induced myopathy. BMJ 2008; 337: a2286. 


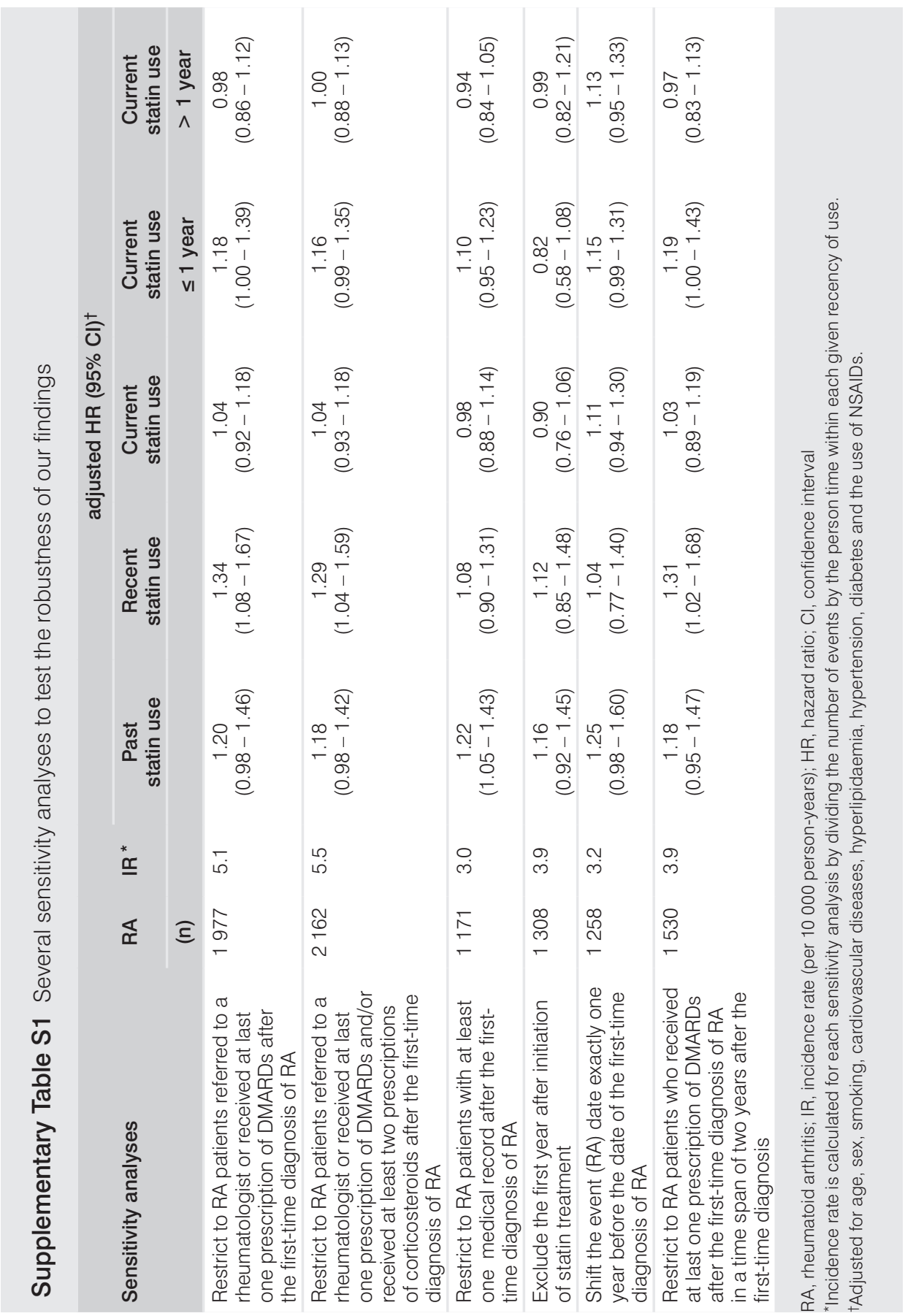




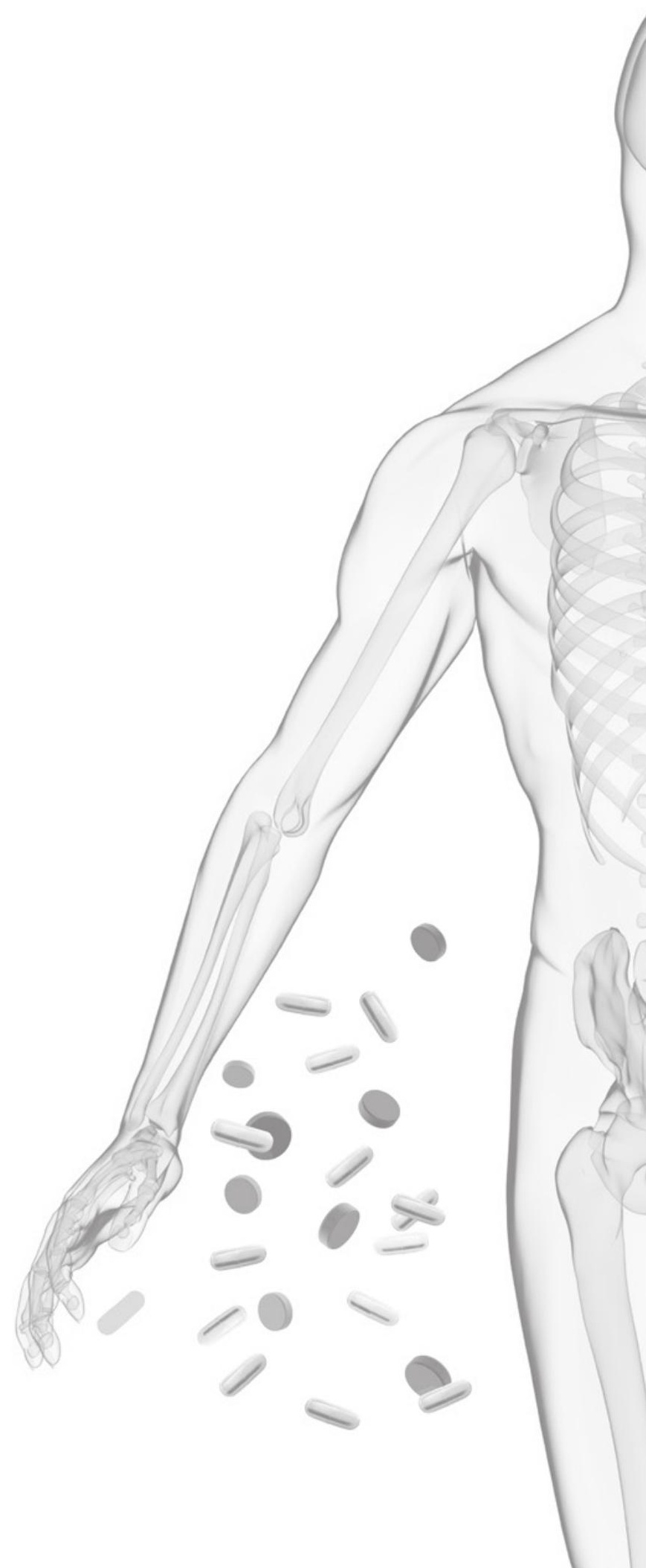




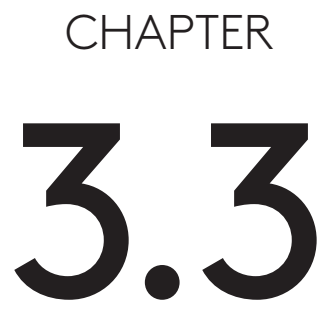

\section{ANGIOTENSIN-CONVERTING ENZYME INHIBITORS OR ANGIOTENSIN II RECEPTOR BLOCKERS AND THE RISK OF DEVELOPING RHEUMATOID ARTHRITIS IN ANTI-HYPERTENSIVE DRUG USERS}

H. J. I. de Jong, R. J. Vandebriel, S.R.F. Saldi, L. van Dijk, H. van Loveren, JW. Cohen Tervaert, O. H. Klungel 


\section{ABSTRACT}

\section{Purpose}

Angiotensin-converting enzyme (ACE) inhibitors and angiotensin II receptor blockers (ARBs) are effective in the treatment of cardiovascular disease. Next to effects on hypertension and cardiac function, these drugs have anti-inflammatory and immunomodulating properties which may either facilitate or protect against the development of autoimmunity, potentially resulting in autoimmune diseases. Therefore, we determined in the current study the association between ACE inhibitor and ARB use and incident rheumatoid arthritis (RA).

\section{Methods}

A matched case-control study was conducted among patients treated with antihypertensive drugs using the Netherlands Information Network of General Practice (LINH) database in 2001-2006. Cases were patients with a first-time diagnosis of RA. Each case was matched to five controls for age, sex, and the index date, which was selected one year before the first diagnosis of RA. ACE inhibitor and ARB exposure was considered to be any prescription issued in the period before the index date. Logistic regression analysis was used to estimate odds ratios (ORs) and their 95\% confidence intervals $(\mathrm{Cl})$.

\section{Results}

Our study included 211 cases and 667 matched controls. After controlling for potential confounders, ever use of ACE inhibitors or ARBs was not associated with incident RA (adjusted ORs, 0.99 (95\% Cl 0.55 to 1.79) and 1.02 (95\% Cl 0.67 to 1.56), respectively). The adjusted ORs for current and past use of ACE inhibitors were 1.18 (95\% Cl 0.75 to 1.85 ) and $0.61(95 \% \mathrm{Cl} 0.28$ to 1.35). For current and past use of ARBs, these adjusted ORs were 1.40 (95\% Cl 0.80 to 2.45$)$ and 0.29 (95\% Cl 0.05 to 1.67), respectively. No duration and dose-effect relationship was observed.

\section{Conclusions}

ACE inhibitor or ARB use is not associated with incident RA. 


\section{INTRODUCTION}

Angiotensin-converting enzyme (ACE) inhibitors and angiotensin II receptor blockers (ARBs) have been reported to reduce mortality and morbidity from cardiovascular events among patients with hypertension, renal, and cardiovascular diseases 1-7. In addition to their effects on blood pressure, cardiac function, and antiproteinuric effect, ACE inhibitors and ARBs have anti-inflammatory and immunomodulating properties 8-11. Open-label studies with rheumatoid arthritis (RA) patients and experimental studies in collagen-induced arthritis suggested a beneficial effect of ACE inhibitors and ARBs in arthritis 12-15. Similarly, ACE inhibitors and ARBs reduce the incidence and severity of experimental autoimmune myocarditis 16 . Otherwise, it has been postulated that the immunomodulating effects of ACE inhibitors may facilitate autoimmune responses 17,18. Indeed, several case reports have suggested that ACE inhibitors may facilitate the development of autoantibodies ${ }^{19-21}$ and autoimmune diseases such as lupus-like syndrome, vasculitis and pemphigus 22-42. Furthermore, one patient with ARB-associated pemphigus has been reported 43 .

The proposed mechanisms by which ACE inhibitors or ARBs may facilitate and be protective for RA are unclear. The anti-inflammatory effects of ACE inhibitors and ARBs are likely due to changes in cellular immunity 8 and to inhibiting adhesion molecule upregulation, chemotaxis, and the release of cytokines by immune cells ${ }^{44-46}$. As a result, ARBs and ACE inhibitors may interfere with leukocyteendothelial cell adhesion and thereby prevent the influx of inflammatory cells in the inflamed synovial membrane and at the cartilage-pannus junction.

Importantly, Shao et al. showed in a study with angiotensin-II-infused hypertensive rats elevated levels of the T helper (Th) cells 1 cytokine interferon- $\gamma$ and decreased levels of Th2 cytokine IL-4. By administering in these rats an ARB, the imbalance of Th subsets was corrected 47 . Furthermore, Platten et al. demonstrated in an animal model of experimental autoimmune encephalitis, mimicking multiple sclerosis, that ACE inhibitors modulate this disease by suppressing autoreactive Th1 and Th17 cells and promoting T-regulatory cells ${ }^{8}$. Otherwise, Coelho dos Santos et al. found that ACE inhibitors increase the intensity of Trypanosoma cruzi infection of human monocytes and decreases the expression of the modulatory cytokine IL-10 while inducing Th17 cells 17 and hence may promote RA, psoriasis and psoriatic arthritis 48-50. Finally, studies have shown that captopril blocks activation-induced apoptosis in T cells 18,51 and hence may interfere with clonal deletion and disturb the maintenance of self-tolerance, thus facilitating autoimmunity 17,18 .

The objective of our study was to investigate whether the use of ACE inhibitors or ARBs increase or decrease incident RA in a large observational study. 


\section{METHODS}

\section{Study population}

Data for this study were obtained from the Netherlands Information Network of General Practice (LINH), a database derived from general practices that records data on morbidity, drug prescriptions and referrals on a continuous basis in electronic medical records. The LINH includes 350000 patients who were registered at 85 practices from 2001 through 2006 52. Prescription data were classified according to the Anatomical Therapeutic and Chemical (ATC) classification 53 , and morbidity was coded by using the International Classification of Primary Care (ICPC) scheme 54. The study was carried out according to Dutch legislation on privacy. The privacy regulation of the study was approved by the Dutch Data Protection Authority. According to Dutch legislation, neither obtaining informed consent nor approval by a medical ethics committee is obligatory for observational studies.

\section{Study design}

We performed a nested, matched case-control study among patients treated with anti-hypertensive drugs. Under our definition of anti-hypertensive drugs, we included miscellaneous anti-hypertensive drugs (ATC code C02), thiazide diuretics (ATC code C03), beta blocking agents (ATC code C07), calcium channel blockers (ATC code C08), ACE inhibitors (ATC code C09A/C09B), and ARBs (ATC code C09C/C09D).

\section{Case definition}

All patients who were first diagnosed with RA and were registered with a general practitioner (GP) for at least one year at the index date were included in the study. Subsequent diagnoses of RA were disregarded. The date exactly one year before the date of first-time diagnosis of RA was subsequently used as the index date, because the diagnosis is usually made after the disease has been symptomatic for some period 55,56 . When the index date was shifted one year before the first-time diagnosis of RA, patients still had to have a medical history of at least one year before this new index date. Patients were considered as having a diagnosis of RA if the first-time diagnosis registered by GP was verified by a referral to a rheumatologist by the use of at least one prescription of disease modifying anti-rheumatoid drug (DMARD) or two or more prescriptions of systemic corticosteroids after the index date 57.

Patients were excluded from the study if they had a medical record of ankylosing spondylitis (ICPC code L88.2) or received at least one prescription of DMARDs before the index date, if they had a diagnosis of RA by the GP with no confirmation according to the mentioned criteria, or if they had no registered medical history for one year 57 . 


\section{Control selection}

Of the anti-hypertensive drug users, five controls were matched to each RA case on age (within five years), sex and the index date. Controls were required to be registered at least one year in the general practice before the index date to minimise information bias. The exclusion criteria used in case selection were applied to controls.

\section{Definition of exposure}

Using data on prescription dispensing date and days supplied, we determined the exposure to ACE inhibitors and ARBs for each patient in the study. All the patients were users of at least one anti-hypertensive drug. Current use was defined as receiving at least one prescription of ACE inhibitors and ARBs within six months before the index date, and past use was defined as receiving a prescription of ACE inhibitors and ARBs but discontinued treatment more than six months before the index date. Patients, who were current or past users of ACE inhibitors and ARBs simultaneously, were categorised as a separate group. Patients who were not users of ACE inhibitors or ARBs but were users of another anti-hypertensive drug such as thiamine diuretics, calcium antagonists and beta blockers were used as the reference category. The expected duration of ACE inhibitor and ARB use until the index date was based on treatment time and prescribed drug supply (determined by the number of prescribed tablets), and was divided into three categories: [1] 1-365 days, [2] 366-1095 days, and [3] more than 1095 days. We calculated the dose of ACE inhibitors and ARBs according to the defined daily dose (DDD), which is the average daily dose of a drug for its main indication in adults ${ }^{51}$ (1 DDD corresponds with captopril $50 \mathrm{mg}$, enalapril $10 \mathrm{mg}$, lisinopril $10 \mathrm{mg}$, perindopril $4 \mathrm{mg}$, ramipril $2.5 \mathrm{mg}$, quinapril $15 \mathrm{mg}$, fosinopril $15 \mathrm{mg}$, losartan $50 \mathrm{mg}$, valsartan $80 \mathrm{mg}$, irbesartan $0.15 \mathrm{~g}$, candesartan $8 \mathrm{mg}$, telmisartan $40 \mathrm{mg}$ and olmesartan medoxomil $20 \mathrm{mg}$ ). Based on almost equal distributions of ACE inhibitor and ARB use, ACE inhibitor exposure was categorised into three categories of less than 0.75 DDD per day, 0.75-1.25 DDD per day, and more than 1.25 DDD per day, and ARB exposure was categorised as less than 1.0 DDD per day and more than 1.0 DDD per day to assess a potential dose-response relationship.

\section{Potential confounders}

Potential confounders included prescriptions within six months preceding the index date for non-steroidal anti-inflammatory drugs (NSAIDs), corticosteroids, proton pump inhibitors, statins, aspirin, antibiotics, hormone replacement therapy, antidepressants, calcium antagonists and thiazide diuretics, and co-morbidities including diabetes, hyperlipidaemia, cardiovascular disease and chronic obstructive pulmonary disease (COPD) 58. Patients were classified as diabetic when they had a diagnosis of diabetes (identified by ICPC codes T89 or T90) or when they received anti-diabetic 
therapy before the index date. Hyperlipidaemia was considered present when the patient had a medical record of hyperlipidaemia (identified by ICPC code T93) or used lipid-lowering drugs prior to the index date. COPD and cardiovascular disease were identified by ICPC code R95 and ICPC codes K71, K73-K84, K89, K90, K92-K96 and $\mathrm{K} 99$, respectively.

\section{Statistical analysis}

Continuous variables were expressed as mean \pm standard deviation, and categorical variables were expressed as frequencies and percentages. For baseline characteristics, continuous data were analysed by Student's t test and categorical data by Chi-square test or Fisher exact test when appropriate. Logistic regression conditioned on the matching factors was used to study the association between ACE inhibitor and ARB use and the risk of RA. Odds ratios and 95\% confidence intervals (95\% Cls) were estimated. We evaluated the effects of ever use, current and past use, and specific type, duration and DDDs of ACE inhibitor and ARB on the risk of developing RA. In addition to controlling for age, sex and calendar time by matching, estimates were adjusted for the mentioned confounders. Furthermore, we evaluated the confounding effects of aspirin, statins, antibiotics, hormone replacement therapy, corticosteroids, antidepressants and thiazide diuretics, and diabetes, hyperlipidaemia and COPD, but we did not include these covariates in the regression models because we did not observe more than $10 \%$ change in the estimate of the exposure-outcome association by adding each time one of these variables into the model ${ }^{59}$. A p value of $\leq 0.05$ was considered statistically significant. We used SAS version 9.2 (SAS Institute, Cary, North Carolina, USA) to analyse the data.

We performed a sensitivity analysis where we investigated the influence of thiazide diuretics on the association between ACE inhibitors/ARB and RA because several studies have demonstrated the association between thiazide diuretics and autoimmune reactions 60,61 . To explore the influence of inclusion of prevalent cases in the study, we performed two sensitivity analyses where we shifted the index date exactly two and three years before the first-time diagnosis of RA. Two sensitivity analyses were performed to examine the effect of misclassification of patients by changing the definition of the outcome (RA). First, we varied the definition of the outcome (RA), specified as first-time diagnosis of RA and a referral to a rheumatologist and a prescription of DMARDs after the index date (irrespective of the use of corticosteroids), a definition used in another study 62 . Second, we investigated the influence of the inclusion of patients with psoriatic arthritis and performed an analysis that excluded patients with a medical record for psoriasis. 


\section{RESULTS}

The study population consisted of 57457 patients who used at least one prescription of anti-hypertensive drugs during the study period from 2001 through 2006. Of those, 877 patients had a first-time diagnosis of RA. Of these RA patients, 72 patients were excluded because they used at least one prescription of DMARDs before the index date and 269 patients because they had no minimum registered medical history of one year. In addition, 325 patients were excluded from the study because the first-time diagnosis of RA by the GP was not confirmed according to the predefined criteria. After these exclusions, we identified 211 eligible RA patients that were matched to 667 controls.

Characteristics of the study population at the index date are described in Table 1. The average age of the study population was 65 years, and approximately $67 \%$ were women. RA patients used more often corticosteroids, NSAIDs, antidepressants and proton pump inhibitors, and were more often diagnosed with COPD than were controls. The duration of registration with the GP was approximately 11.2 years in RA patients and 10.5 years in controls.

Table 1 Characteristics of study population at the index date

\begin{tabular}{lrrc}
\hline Characteristics & $\begin{array}{c}\text { Case } \\
(\mathbf{n = 2 1 1 )}\end{array}$ & $\begin{array}{c}\text { Control } \\
(\mathbf{n}=667)\end{array}$ & p value* \\
\hline Mean Age in years (SD) & $69.6 \pm 11.2$ & $68.3 \pm 11.8$ & NS \\
\hline Sex (\%) & $66(31.3)$ & $195(29.2)$ & NS \\
\hline Male & $145(68.7)$ & $472(70.8)$ & NS \\
\hline Female & & & \\
\hline Duration registration at general practice in years (\%) & & & NS \\
\hline $1-5.99$ & $53(25.1)$ & $197(29.5)$ & NS \\
\hline $6-11.99$ & $93(44.1)$ & $286(42.9)$ & NS \\
\hline$\geq 12$ & $65(30.8)$ & $184(27.6)$ & \\
\hline Disease history before the index date (\%) & & & NS \\
\hline Hypertension & $134(63.5)$ & $421(63.1)$ & NS \\
\hline Diabetes & $31(14.7)$ & $125(18.7)$ & N \\
\hline Cardiovascular disease & $95(45.0)$ & $219(32.8)$ & 0.001 \\
\hline Hyperlipidaemia & $58(27.5)$ & $165(24.7)$ & NS \\
\hline Hepatic disease & $6(2.8)$ & $14(2.1)$ & NS \\
\hline Renal disease & $4(1.9)$ & $8(1.2)$ & NS \\
\hline COPD & $31(14.7)$ & $27(4.1)$ & $<0.001$ \\
\hline
\end{tabular}


Table 1 Characteristics of study population at the index date

\begin{tabular}{|c|c|c|c|}
\hline Characteristics & $\begin{array}{c}\text { Case } \\
(n=211)\end{array}$ & $\begin{array}{l}\text { Control } \\
(n=667)\end{array}$ & $p$ value* \\
\hline \multicolumn{4}{|l|}{ Disease history before the index date (\%) } \\
\hline Cancer & $9(4.3)$ & $33(5.0)$ & NS \\
\hline Inflammatory bowel syndrome & $2(0.3)$ & $2(1.0)$ & NS \\
\hline \multicolumn{4}{|l|}{ Drug use 6 months before the index date (\%) } \\
\hline Corticosteroids & $34(16.1)$ & $16(2.4)$ & $<0.001$ \\
\hline NSAIDs & $71(33.7)$ & $48(7.2)$ & $<0.001$ \\
\hline \multicolumn{4}{|l|}{ Anti-hypertensive drugs } \\
\hline Miscellaneous anti-hypertensive drugs & $0(0.0)$ & $8(1.2)$ & NS \\
\hline Thiazide diuretics & $95(45.0)$ & $277(41.5)$ & NS \\
\hline Beta blockers & $95(45.0)$ & $292(43.8)$ & NS \\
\hline Calcium channel blockers & $48(22.8)$ & $109(16.3)$ & NS \\
\hline ACE inhibitors & $52(24.6)$ & $155(23.2)$ & NS \\
\hline ARBs & $32(15.2)$ & $76(11.4)$ & NS \\
\hline Aspirin & $28(13.3)$ & $87(13.0)$ & NS \\
\hline Anti-diabetics & $23(10.9)$ & $85(12.7)$ & NS \\
\hline Lipid-lowering agents & $50(23.7)$ & $132(19.8)$ & NS \\
\hline HRT & $7(3.3)$ & $20(3.0)$ & NS \\
\hline Antibiotics & $40(19.0)$ & $83(12.4)$ & 0.02 \\
\hline Anti-psychotics & $1(0.5)$ & $6(0.9)$ & NS \\
\hline Antidepressants & $23(10.9)$ & $33(5.0)$ & 0.002 \\
\hline PPI & 47 (22.3) & $52(7.8)$ & $<0.001$ \\
\hline
\end{tabular}

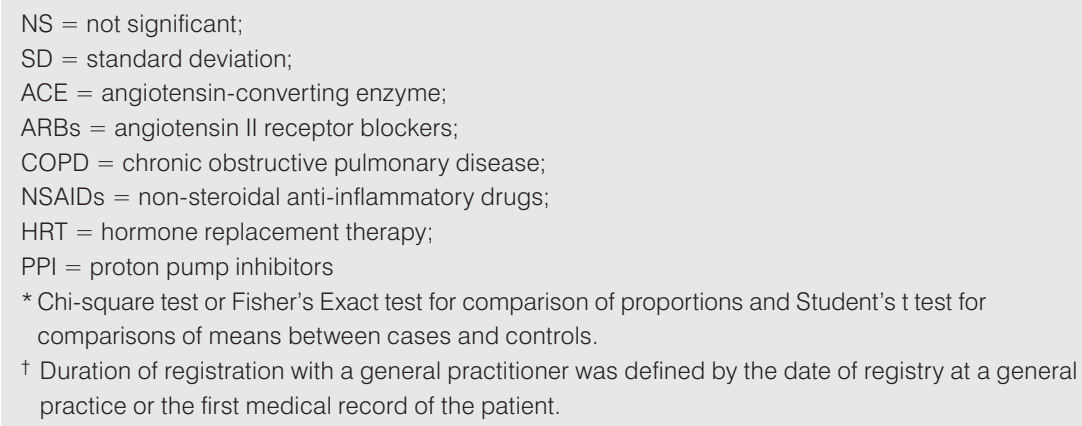

Table 2 shows that ACE inhibitor use or ARB use prior to the index date was not associated with incident RA (adjusted odds ratios (ORs), 1.02 (95\% Cl 0.59 to 1.78)) and (adjusted OR, 1.02 (95\% Cl 0.69 to 1.51)), respectively. The adjusted ORs of 
incident RA associated with current and past use of ACE inhibitors were 1.15 (95\% Cl 0.76 to 1.74) and 0.70 (95\% $\mathrm{Cl} 0.34$ to1.47), respectively. Current and past use of ARBs were not associated with incident RA (adjusted ORs, 1.39 (95\% Cl 0.82 to 2.33) and 0.29 (95\% $\mathrm{Cl} 0.06$ to 1.96), respectively). In addition, current use and past use of the combination therapy of ACE inhibitors and ARBs were not associated with incident RA. No relation between the duration and intensity of use of ACE inhibitors or ARBs, expressed as days of use and numbers of DDD, and the risk of developing RA was observed (Table 3). Stratifying results according to the type of ACE inhibitor and ARB use showed no risk differences between ACE inhibitor or ARB users and non-users (data not shown).

Table 2 Association between use of angiotensin-converting enzyme inhibitors and angiotensin II receptor blockers and the risk of rheumatoid arthritis

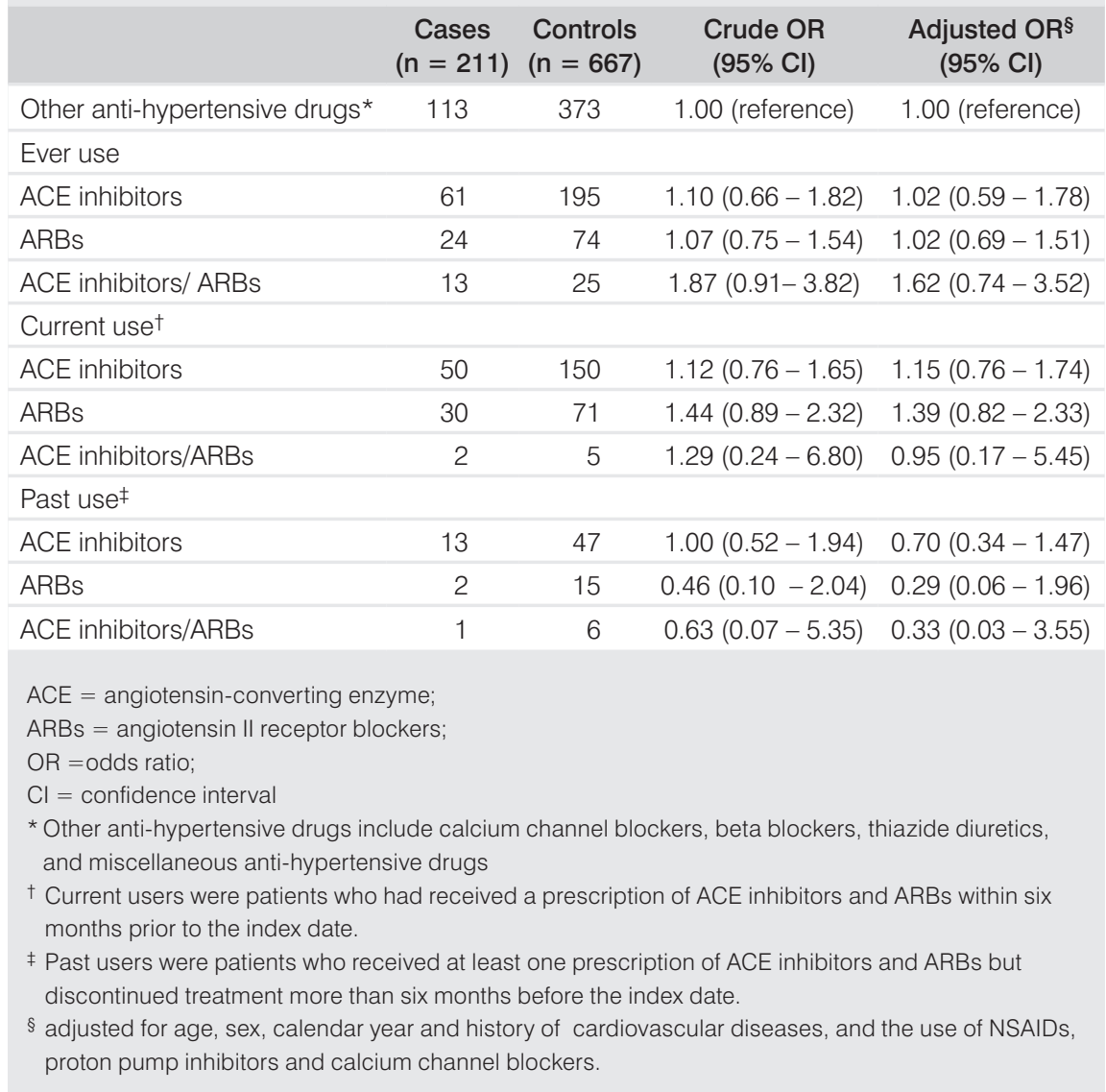


Table 3 Use of different dosages of angiotensin-converting enzyme inhibitors and the risk of rheumatoid arthritis

\begin{tabular}{|c|c|c|c|c|}
\hline & $\begin{array}{c}\text { Cases } \\
(n=211)\end{array}$ & $\begin{array}{l}\text { Controls } \\
(n=667)\end{array}$ & $\begin{array}{c}\text { Crude OR } \\
(95 \% \mathrm{Cl})\end{array}$ & $\begin{array}{l}\text { Adjusted OR }{ }^{\dagger} \\
(95 \% \mathrm{Cl})\end{array}$ \\
\hline Other antihypertensive drugs* & 113 & 373 & 1.00 (reference) & 1.00 (reference) \\
\hline \multicolumn{5}{|l|}{ Use of ACE inhibitors, days } \\
\hline $1-365$ & 19 & 61 & $1.04(0.59-1.81)$ & $0.90(0.50-1.62)$ \\
\hline $366-1095$ & 19 & 69 & $0.89(0.51-1.56)$ & $0.84(0.47-1.50)$ \\
\hline$>1095$ & 23 & 65 & $1.27(0.74-2.16)$ & $1.24(0.72-2.16)$ \\
\hline \multicolumn{5}{|l|}{ Use of ARBs, days } \\
\hline $1-365$ & 8 & 33 & $0.85(0.38-1.90)$ & $0.80(0.35-1.84)$ \\
\hline $366-1095$ & 10 & 29 & $1.12(0.53-2.36)$ & $0.93(0.42-2.05)$ \\
\hline$>1095$ & 6 & 12 & $1.82(0.66-5.06)$ & $1.64(0.57-4.69)$ \\
\hline \multicolumn{5}{|l|}{ Use of ACE inhibitors, DDD } \\
\hline$\leq 0.75$ & 17 & 64 & $0.90(0.50-1.62)$ & $0.83(0.44-1.58)$ \\
\hline $0.75-1.25$ & 27 & 70 & $1.34(0.82-2.21)$ & $1.28(0.75-2.21)$ \\
\hline$>1.25$ & 17 & 61 & $0.94(0.52-1.67)$ & $0.96(0.52-1.78)$ \\
\hline \multicolumn{5}{|l|}{ Use of ARBs, DDD } \\
\hline$\leq 1$ & 17 & 61 & $0.96(0.53-1.72)$ & $0.84(0.44-1.59)$ \\
\hline$>1$ & 7 & 13 & $1.71(0.66-4.43)$ & $2.03(0.72-5.76)$ \\
\hline $\begin{array}{l}\text { ACE = angiotensin-converting en } \\
\text { ARBs = angiotensin II receptor bl } \\
\mathrm{OR}=\text { odds ratio; } \\
\mathrm{Cl}=\text { confidence interval; } \\
\mathrm{DDD}=\text { defined daily dose } \\
\text { * Other anti-hypertensive drugs in } \\
\text { and miscellaneous anti-hyperter } \\
\text { † adjusted for age, sex, calendar } \\
\text { proton pump inhibitors and calc }\end{array}$ & $\begin{array}{l}\text { clude calciun } \\
\text { sive drugs } \\
\text { ear and histo } \\
\text { ium channel }\end{array}$ & $\begin{array}{l}\text { n channel blc } \\
\text { ory of cardio } \\
\text { blockers. }\end{array}$ & $\begin{array}{l}\text { ckers, beta blockers, } \\
\text { vascular diseases, anc }\end{array}$ & $\begin{array}{l}\text { thiazide diuretics, } \\
\text { the use of NSAIDs, }\end{array}$ \\
\hline
\end{tabular}

\section{Sensitivity analyses}

The risk of RA associated with ACE inhibitors or ARBs was similar for subjects using thiazide diuretics and subjects not using thiazide diuretics. When we shifted the index date two and three years before the first-time diagnosis of RA, we found no association between ACE inhibitor and ARB use and incident RA (data not shown), and no difference in the distribution of NSAID and corticosteroid use between cases and controls was observed. When the analysis was restricted to RA patients referred to a rheumatologist or received at least one prescription of a DMARD after the index date, 
the adjusted ORs for ACE inhibitor and ARB use and the risk of developing RA were 0.92 (95\% Cl 0.45 to 1.87 ) and 0.89 (95\% Cl 0.36 to 1.79), respectively. To exclude possible bias by accidental inclusion of patients with psoriatic arthritis among RA patients, we repeated the analysis after removing the patients who had a recorded diagnosis of psoriasis. Results were consistent with all other analyses (adjusted OR ACE inhibitor, 1.03 (95\% Cl 0.61 to 1.85), adjusted OR ARB, 1.06 (95\% Cl 0.68 to 1.53) and adjusted OR ACE inhibitor and ARB, 1.63(95\% Cl 0.74 to 3.57$)$ ).

\section{DISCUSSION}

The present study did not demonstrate an association between ACE inhibitor and ARB use and the risk of developing RA. The effect was consistent when we defined the exposure to ACE inhibitors and ARBs as current and past use and duration of use. Furthermore, no dose-response effect was observed. Thus, our data do not support the hypothesis that ACE inhibitors and ARBs either facilitate or protect against RA. To our knowledge, this is the first observational study investigating the influence of ACE inhibitor and ARB use and incident RA. Evidence for the association between ACE inhibitor and/or ARB use and the occurrence of autoimmune diseases was derived from case reports of lupus-like disease, vasculitis and pemphigus 22-43. In these case reports, the commonly prescribed ACE inhibitors such as captopril and enalapril were often reported as a suspected drug for the occurrence of these autoimmune diseases. In our study, however, we observed no difference in the risk estimates of individual ACE inhibitors and the risk of developing RA.

Several studies have shown the beneficial effects of ARB and ACE inhibitor use in animal models of induced arthritis 13,14,63,64. Furthermore, in an open study of patients with active RA treated with captopril, reductions in joint symptoms, the number of swollen joints and reduced levels of C-reactive protein (CRP) were found ${ }^{12}$. In addition, in an open study using pentopril, no clinical improvement in patients with RA was observed, although CRP levels decreased 65. Furthermore, ARB use in RA patients was found to be associated with a significant reduction in erythrocyte sedimentation rate ${ }^{15}$. The findings are, however, inconclusive 15,66.

Several limitations of our study should be considered in the interpretation of these results. First, the definition of RA in our study was based on GPs' diagnosis, a referral to a rheumatologist, or prescriptions of DMARDs and/or corticosteroids after the index date. We may have used a relatively sensitive, but nonspecific, diagnosis of RA because no specific ICPC codes were available for other rheumatic diseases, e.g., psoriatic arthritis. Furthermore, no data on examinations such as the presence of anti-cyclic citrullinated peptide antibodies or X-ray of hands and feet were available for making a diagnosis of RA. Therefore, false positives, meaning that not all cases 
were true cases of RA, may have diluted the results of our study, and we may have underestimated the OR of RA. However, our results remained unchanged when analyses were restricted to RA patients referred to a rheumatologist or those who received DMARDs after their first-time diagnosis, or when we excluded patients with a diagnosis for psoriasis, or when we modified the index date exactly two and three years before the first-time diagnosis of RA. Because of limited power of our study population, we used an index date exactly one year before the first-time diagnosis of RA. When the analysis was restricted to RA patients referred to a rheumatologist and received at least one prescription of a DMARD after the index date, the results were consisted with all other analyses. Our findings indicate no association between ACE inhibitor and ARB use and incident RA when we used other criteria for defining RA in our study.

Second, our study was observational, and patients were not randomly assigned to anti-hypertensive therapy. Physicians and patients selected anti-hypertensive drug therapies, and this may have introduced bias. We believe, however, that it is unlikely that this bias has influenced our results because no differences between cases and controls in the prescriptions of other anti-hypertensive drugs were observed.

Third, misclassification of ACE inhibitor and ARB use may be a concern in this study because we used computerised prescription data from the GP. Patients may have been non-adherent to the treatment of ACE inhibitors or ARBs and therefore could have used less ACE inhibitors or ARBs than prescribed. However, this misclassification is likely to be non-differential between cases and controls and may have resulted in an underestimation of the association between use of ACE inhibitors and/or ARBs and RA.

Fourth, selection bias may have been introduced in this study because GPs could have paid more attention to co-morbidities of RA patients. Yet, the average duration in the registry is almost equal between RA and controls, and therefore; the patients in our study had similar opportunities to have been prescribed an ACE inhibitor or ARB. Further limitations include the lack of information on dietary intake, physical activity and smoking, and limited data on other examinations (e.g. blood pressure, lipid levels) 67-69.

From the approximately 57457 eligible patients who were taking anti-hypertensive medication, we ultimately included 211 RA patients in our study. Due to several exclusions, our results may not necessarily be extrapolated to all treated hypertensive patients. In addition, the group of users of ACE inhibitors and ARBs in our study was relatively small.

Strengths of our study include the use of different classes of anti-hypertensive drugs and the use of a computerised database, allowing us to use routinely recorded medical and prescription data from GPs. Consequently, recall bias was minimised. Another strength of our study is its inclusion of patients who were prescribed an an- 
ti-hypertensive drug. By including only these patients, we have ensured that prognostic factors such as cardiovascular risk factors (e.g. hypertension) were almost evenly distributed between the RA patients and controls 70 .

Furthermore, because hypertension may be considered as an immune-mediated disease 71,72 hypertension-related immunological abnormalities relevant for the development of RA were probably also equally distributed between both groups. Nonetheless, hypertension is more prevalent in RA patients than in the general population ${ }^{73}$, and theoretically, in view of their immunomodulating capacities, the use of ACE inhibitors and/or ARBs might prevent the development of RA.

In conclusion, this study suggests that there is no association between incident RA and ACE inhibitor or ARB use. Our results indicate that the beneficial effects of ACE inhibitors and ARBs studied in patients with RA were not observed among hypertensive patients without clinically manifest RA. Furthermore, our study does not underline findings from individual case reports suggesting that ACE inhibitors and ARBs may facilitate the development of autoimmune diseases. Based on these findings, we believe that both ACE inhibitors and ARBs are considered to be safe for patients with a risk of developing RA. Further research using larger sample size and a prospective study design are needed to confirm our findings. It is of interest to replicate the design of this study to assess the association with psoriasis and psoriatic arthritis.

\section{Key points}

Use of ACE inhibitors or ARBs is not associated with incident RA

In contrast to the beneficial effects of ACE inhibitors and ARBs studied in patients with RA, we did not observe effects on the incidence of RA among patients initially free of RA.

This study does not underline findings from individual case reports, suggesting that ACE inhibitors or ARBs may not facilitate the development of autoantibodies and/or autoimmune disease.

Based on our findings, both ACE inhibitors and ARBs are considered to be safe for patients with a risk of developing RA.

\section{Acknowledgements}

The authors thank the staff of LINH for their help in the data collection and data extraction and Dr R. Janssen for her helpful suggestions. 


\section{REFERENCES}

1. Montecucco F, Mach F. Statins, ACE inhibitors and ARBs in cardiovascular disease. Best Pract Res Clin Endocrinol Metab 2009; 23(3): 389-400.

2. Yusuf S, Sleight P, Pogue J, Bosch J, Davies R, Dagenais G. Effects of an angiotensin-converting-enzyme inhibitor, ramipril, on cardiovascular events in high-risk patients. The Heart Outcomes Prevention Evaluation Study Investigators. N Engl J Med 2000; 342(3): 145-53.

3. Fox KM, EURopean trial. On reduction of cardiac events with Perindopril in stable coronary Artery disease Investigators. Efficacy of perindopril in reduction of cardiovascular events among patients with stable coronary artery disease: randomised, double-blind, placebo-controlled, multicentre trial (the EUROPA study). Lancet 2003; 362(9386): 782-8.

4. Dahlöf B, Devereux RB, Kjeldsen SE, et al. Cardiovascular morbidity and mortality in the Losartan Intervention For Endpoint reduction in hypertension study (LIFE): a randomised trial against atenolol. Lancet 2002; 359(9311): 995-1003.

5. Pfeffer MA, Swedberg K, Granger CB, et al. Effects of candesartan on mortality and morbidity in patients with chronic heart failure: the CHARM-Overall programme. Lancet 2003; 362(9386): 759-66.

6. Chobanian AV, Bakris GL, Black HR, et al. Seventh report of the Joint National Committee on Prevention, Detection, Evaluation, and Treatment of High Blood Pressure. Hypertension 2003; 42(6): 1206-52.

7. MacKinnon M, Shurraw S, Akbari A, Knoll GA, Jaffey J, Clark HD. Combination therapy with an angiotensin receptor blocker and an ACE inhibitor in proteinuric renal disease: a systematic review of the efficacy and safety data. Am J Kidney Dis 2006; 48(1): 8-20.

8. Platten M, Youssef S, Hur EM, et al. Blocking angiotensin-converting enzyme induces potent regulatory T cells and modulates TH1- and TH17-mediated autoimmunity. Proc Natl Acad Sci U S A 2009; 106(35): 14948-53.

9. Stegbauer J, Lee DH, Seubert S, et al. Role of the renin-angiotensin system in autoimmune inflammation of the central nervous system. Proc Natl Acad Sci U S A 2009; 106(35): 14942-7.

10. Abd Alla J, Langer A, Elzahwy SS, Arman-Kalcek G, Streichert T, Quitterer U. Angiotensin-converting enzyme inhibition down-regulates the pro-atherogenic chemokine receptor 9 (CCR9)-chemokine ligand 25 (CCL25) axis. J Biol Chem 2010; 285(30): 23496-505.

11. Fliser D, Buchholz K, Haller H, EUropean Trial on Olmesartan and Pravastatin in Inflammation and Atherosclerosis (EUTOPIA) Investigators. Antiinflammatory effects of angiotensin II subtype 1 receptor blockade in hypertensive patients with microinflammation. Circulation 2004; 110(9): 1103-7.

12. Martin MF, Surrall KE, McKenna F, Dixon JS, Bird HA, Wright V. Captopril: a new treatment for rheumatoid arthritis? Lancet 1984; 1(8390): 1325-8

13. Sagawa K, Nagatani K, Komagata Y, Yamamoto K. Angiotensin receptor blockers suppress antigenspecific T cell responses and ameliorate collagen-induced arthritis in mice. Arthritis Rheum 2005; 52(6): 1920-8

14. Dalbeth N, Edwards J, Fairchild S, Callan M, Hall FC. The non-thiol angiotensin-converting enzyme inhibitor quinapril suppresses inflammatory arthritis. Rheumatology (Oxford) 2005; 44(1): 24-31.

15. Perry ME, Chee MM, Ferrell WR, Lockhart JC, Sturrock RD. Angiotensin receptor blockers reduce erythrocyte sedimentation rate levels in patients with rheumatoid arthritis. Ann Rheum Dis 2008; 67(11): 1646-7.

16. Godsel LM, Leon JS, Wang K, Fornek JL, Molteni A, Engman DM. Captopril prevents experimental autoimmune myocarditis. J Immunol 2003; 171(1): 346-52.

17. Coelho dos Santos JS, Menezes CA, Villani FN, Magalhães LM, Scharfstein J, Gollob KJ, Dutra WO. Captopril increases the intensity of monocyte infection by Trypanosoma cruzi and induces human $T$ helper type 17 cells. Clin Exp Immunol 2010; 162(3): 528-36.

18. Odaka C, Mizuochi T. Angiotensin-converting enzyme inhibitor captopril prevents activation-induced apoptosis by interfering with T cell activation signals. Clin Exp Immunol 2000; 121(3): 515-22.

19. Kallenberg CG, Hoorntje SJ, Smit AJ, Weening JJ, Donker AJ, Hoedemaeker PH, The TH. Antinuclear and antinative DNA antibodies during captopril treatment. Acta Med Scand 1982; 211(4): 297-300. 
20. Reidenberg MM, Case DB, Drayer DE, Reis S, Lorenzo B. Development of antinuclear antibody in patients treated with high doses of captopril. Arthritis Rheum 1984; 27(5): 579-81.

21. Schwartz D, Pines A, Averbuch M, Levo Y. Enalapril-induced antinuclear antibodies. Lancet 1990; 336(8708): 187.

22. Patri P, Nigro A, Rebora A. Lupus erythematosus-like eruption from captopril. Acta Derm Venereol 1985; 65(5): 447-8.

23. Sieber C, Grimm E, Follath F. Captopril and systemic lupus erythematosus syndrome. BMJ 1990; 301(6753): 669

24. Bertin P, Kamdem J, Bonnet C, Arnaud M, Treves R. Captopril-induced lupus. Clin Exp Rheumatol 1993; 11(6): 695.

25. Pelayo M, Vargas V, Gonzales A, Vallano A, Esteban R, Guardia J. Drug-induced lupus-like reaction and captopril. Ann Pharmacother 1993; 27(12): 1541-2.

26. Fernández-Díaz ML, Herranz P, Suárez-Marrero MC, Borbujo J, Manzano R, Casado M. Subacute cutaneous lupus erythematosus associated with cilazapril. Lancet 1995; 345(8946): 398

27. Ratliff NB, 3rd. Captopril induced lupus. J Rheumatol 2002; 29(8): 1807-8.

28. Disdier P, Harle JR, Verrot D, Jouglard J, Weiller PJ. Adult Schönlein-Henoch purpura after lisinopril. Lancet 1992; 340(8825): 985

29. Moots RJ, Keeling PJ, Morgan SH. Adult Schönlein-Henoch purpura after enalapril. Lancet 1992; 340(8814): 304-5.

30. Gonçalves R, Cortez Pinto H, Serejo F, Ramalho F. Adult Schönlein-Henoch purpura after enalapril. J Intern Med 1998; 244(4): 356-7.

31. Gupta S, Gandhi NM, Ferguson J. Cutaneous vasculitis secondary to ramipril. J Drugs Dermatol 2004; 3(1): 81-2.

32. Pinto GM, Lamarão P, Vale T. Captopril-induced pemphigus vegetans with Charcot-Leyden crystals. J Am Acad Dermatol 1992; 27(2 Pt 2): 281-4

33. Kaplan RP, Potter TS, Fox JN. Drug-induced pemphigus related to angiotensin-converting enzyme inhibitors. J Am Acad Dermatol 1992; 26 (2 Pt 2): 364-6.

34. Ruocco V, Satriano RA, Guerrera V. "Two-step" pemphigus induction by ACE-inhibitors. Int J Dermatol 1992; 31(1): 33-6.

35. Kuechle MK, Hutton KP, Muller SA. Angiotensin-converting enzyme inhibitor-induced pemphigus: three case reports and literature review. Mayo Clin Proc 1994; 69(12): 1166-71.

36. Bastiaens MT, Zwan NV, Verschueren GL, Stoof TJ, Nieboer C. Three cases of pemphigus vegetans: induction by enalapril--association with internal malignancy. Int J Dermatol 1994; 33(3): 168-71.

37. Butt A, Burge SM. Pemphigus vulgaris induced by captopril. Br J Dermatol 1995; 132(2): 315-6

38. Buzón E, Pérez-Bernal AM, de la Pe a F, Ríos JJ, Camacho F. Pemphigus foliaceus associated with cilazapril. Acta Derm Venereol 1998; 78(3): 227.

39. Vignes S, Paul C, Flageul B, Dubertret L. Ramipril-induced superficial pemphigus. Br J Dermatol 1996; 135(4): 657-8.

40. Ong CS, Cook N, Lee S. Drug-related pemphigus and angiotensin converting enzyme inhibitors. Australas J Dermatol 2000; 41(4): 242-6.

41. Parodi A, Cozzani E, Milesi G, Drosera M, Rebora A. Fosinopril as a possible pemphigus-inducing drug. Dermatology 2002; 204(2): 139-41.

42. Patterson CR, Davies MG. Pemphigus foliaceus: an adverse reaction to lisinopril. J Dermatolog Treat 2004; 15(1): 60-2.

43. Bae YI, Yun SJ, Lee SC, Park GT, Lee JB. Pemphigus foliaceus induced by an angiotensin II receptor blocker. Clin Exp Dermatol 2008; 33(6): 721-3.

44. Godsel LM, Leon JS, Engman DM. Angiotensin converting enzyme inhibitors and angiotensin II receptor antagonists in experimental myocarditis. Curr Pharm Des 2003; 9(9): 723-35.

45. Sukumaran V, Watanabe K, Veeraveedu PT, et al. Olmesartan, an AT1 antagonist, attenuates oxidative stress, endoplasmic reticulum stress and cardiac inflammatory mediators in rats with heart failure induced by experimental autoimmune myocarditis. Int J Biol Sci 2011; 7(2): 154-67. 
46. Ruiz-Ortega M, Bustos C, Hernández-Presa MA, Lorenzo O, Plaza JJ, Egido J. Angiotensin Il participates in mononuclear cell recruitment in experimental immune complex nephritis through nuclear factor-kappa B activation and monocyte chemoattractant protein-1 synthesis. J Immunol 1998; 161(1): 430-9.

47. Shao J, Nangaku M, Miyata T, Inagi R, Yamada K, Kurokawa K, Fujita T. Imbalance of T-cell subsets in angiotensin II-infused hypertensive rats with kidney injury. Hypertension 2003; 42(1): 31-8.

48. Miossec P. Interleukin-17 and Th17 cells: from adult to juvenile arthritis--now it is serious! Arthritis Rheum 2011; 63(8): 2168-71.

49. Blauvelt A. T-helper 17 cells in psoriatic plaques and additional genetic links between IL-23 and psoriasis. J Invest Dermatol 2008; 128(5): 1064-7.

50. Leipe J, Grunke M, Dechant C, Reindl C, Kerzendorf U, Schulze-Koops H, Skapenko A. Role of Th17 cells in human autoimmune arthritis. Arthritis Rheum 2010; 62(10): 2876-85.

51. Déas O, Dumont C, Mollereau B, et al. Thiol-mediated inhibition of FAS and CD2 apoptotic signaling in activated human peripheral T cells. Int Immunol 1997; 9(1): 117-25.

52. Verheij RA, Van Dijk CE, Stirbu-Wagner I, et al. Landelijk Informatienetwork Huisartsenzorg. Feiten en cijfers over huisartsenzorg in Nederland. Utrecht/Nijmegen, The Netherlands: Nivel/IQ, 2009.

53. Whocc.no [internet]. Oslo: WHO Collaborating Centre for Drug Statistics Methodology; c1996 - [updated 2010 Feb 5; cited 2011 Sep 25]. Available from: http://http://www.whocc.no/atc_ddd_index/.

54. Lamberts H, Wood M. ICPC. International Classification of Primary Care. Oxford: Oxford University Press 1987.

55. Chan KW, Felson DT, Yood RA, Walker AM. The lag time between onset of symptoms and diagnosis of rheumatoid arthritis. Arthritis Rheum 1994; 37(6): 814-20.

56. Palm $\varnothing$, Purinszky E. Women with early rheumatoid arthritis are referred later than men. Ann Rheum Dis 2005; 64(8): 1227-8.

57. De Jong HJ, Klungel OH, Van Dijk L, et al. Use of statins is associated with an increased risk of rheumatoid arthritis. Ann Rheum Dis 2012; 71(5): 648-54

58. Pedersen M, Jacobsen S, Klarlund M, Pedersen BV, Wiik A, Wohlfahrt J, Frisch M. Environmental risk factors differ between rheumatoid arthritis with and without auto-antibodies against cyclic citrullinated peptides. Arthritis Res Ther 2006; 8(4): R133.

59. Mickey RM, Greenland S. The impact of confounder selection criteria on effect estimation. Am J Epidemiol 1989; 129(1): 125-37.

60. Rubin RL. Drug-induced lupus. Toxicology 2005; 209(2): 135-47.

61. Chang C, Gershwin ME. Drugs and autoimmunity--a contemporary review and mechanistic approach. J Autoimmun 2010; 34(3): J266-75.

62. Jick SS, Choi H, Li L, Mclnnes IB, Sattar N. Hyperlipidaemia, statin use and the risk of developing rheumatoid arthritis. Ann Rheum Dis 2009; 68(4): 546-51.

63. Sakuta T, Morita Y, Satoh M, Fox DA, Kashihara N. Involvement of the renin-angiotensin system in the development of vascular damage in a rat model of arthritis: effect of angiotensin receptor blockers. Arthritis Rheum 2010; 62(5): 1319-28.

64. Price A, Lockhart JC, Ferrell WR, Gsell W, McLean S, Sturrock RD. Angiotensin II type 1 receptor as a novel therapeutic target in rheumatoid arthritis: in vivo analyses in rodent models of arthritis and ex vivo analyses in human inflammatory synovitis. Arthritis Rheum 2007; 56(2): 441-7.

65. Bird HA, Le Gallez P, Dixon JS, et al. A clinical and biochemical assessment of a nonthiol ACE inhibitor (pentopril; CGS-13945) in active rheumatoid arthritis. J Rheumatol 1990; 17(5): 603-8.

66. Tikiz C, Utuk O, Pirildar T, et al. Effects of Angiotensin-converting enzyme inhibition and statin treatment on inflammatory markers and endothelial functions in patients with longterm rheumatoid arthritis. J Rheumatol 2005; 32(11): 2095-101.

67. Hazes JM, Dijkmans BA, Vandenbroucke JP, De Vries RR, Cats A. Lifestyle and the risk of rheumatoid arthritis: cigarette smoking and alcohol consumption. Ann Rheum Dis 1990; 49(12): 980-2.

68. Plasqui $\mathrm{G}$. The role of physical activity in rheumatoid arthritis. Physiol Behav 2008; 94(2): 270-5.

69. Sugiyama D, Nishimura K, Tamaki K, Tsuji G, Nakazawa T, Morinobu A, Kumagai S. Impact of smoking as a risk factor for developing rheumatoid arthritis: a meta-analysis of observational studies. Ann Rheum Dis 2010; 69(1): 70-81. 
70. Klungel $\mathrm{OH}$, Martens EP, Psaty BM, et al. Methods to assess intended effects of drug treatment in observational studies are reviewed. J Clin Epidemiol 2004; 57(12): 1223-31.

71. Cohen Tervaert JW. Hypertension: an autoimmune disease? Hypertens Res 2011; 34(4): 443-4.

72. Harrison DG, Guzik TJ, Lob HE, et al. Inflammation, immunity, and hypertension. Hypertension 2011; 57(2): 132-40.

73. Dessein PH, Norton GR. Should patients with RA be aggressively monitored for hypertension? Nat Clin Pract Rheumatol 2008; 4(1): 18-9. 


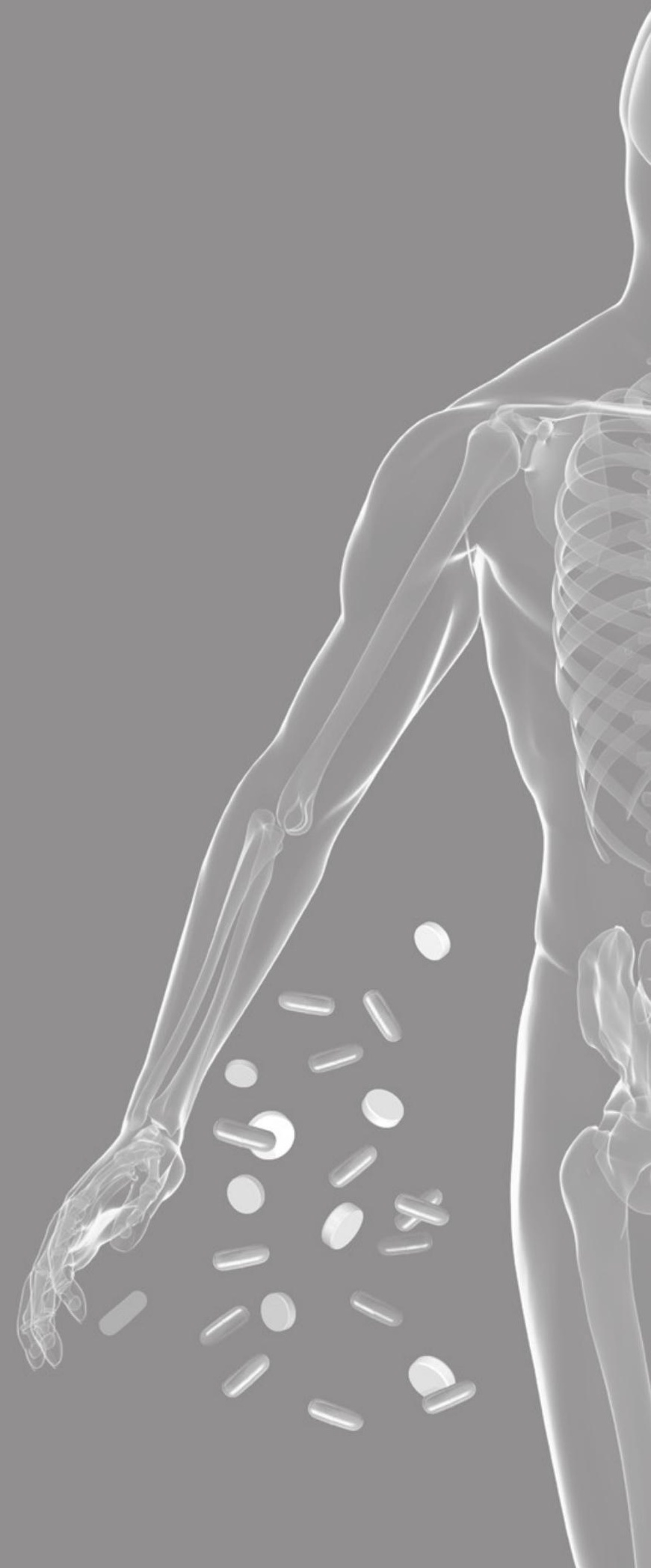




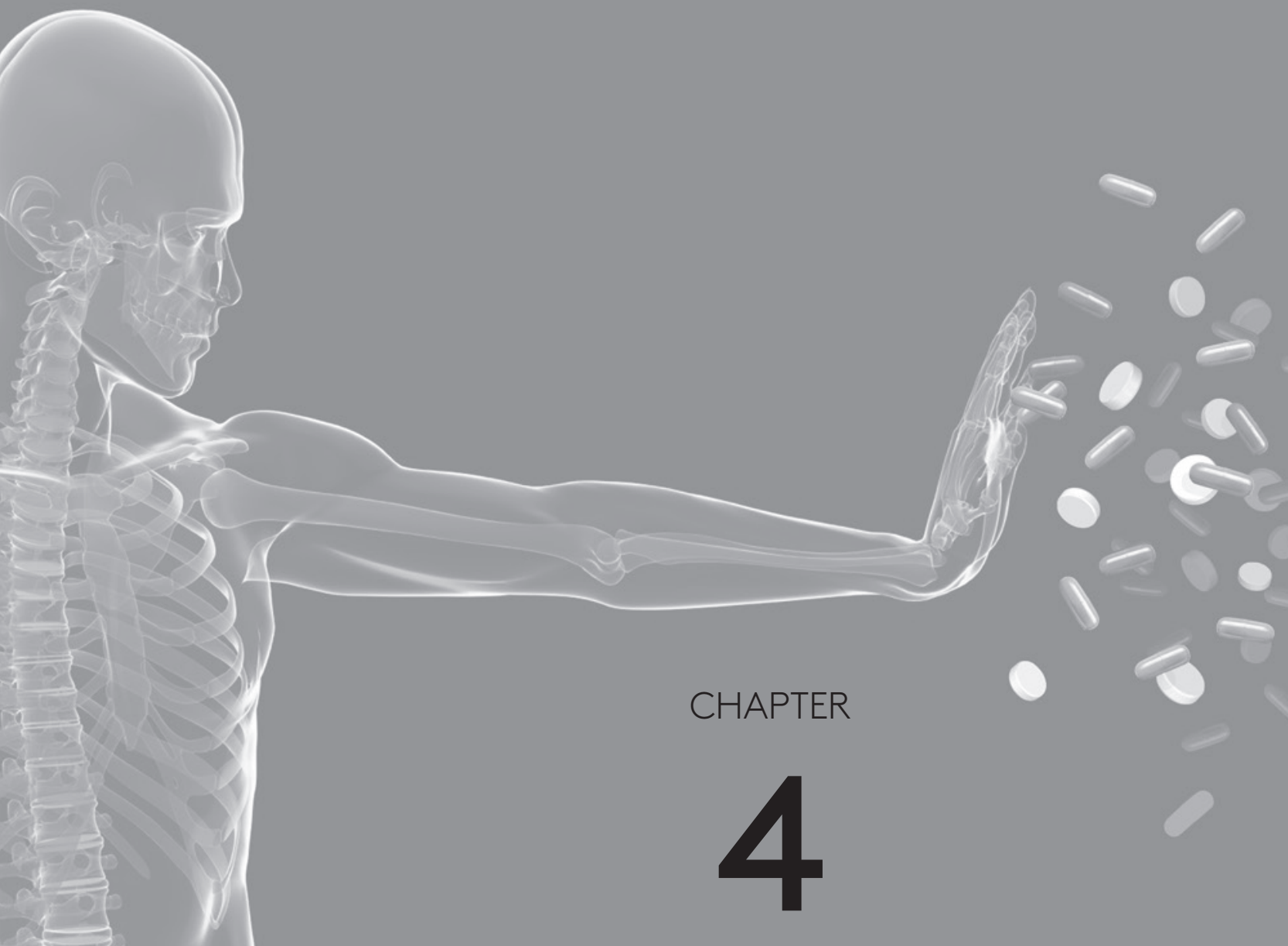

STATIN USE AND AUTOIMMUNITY 


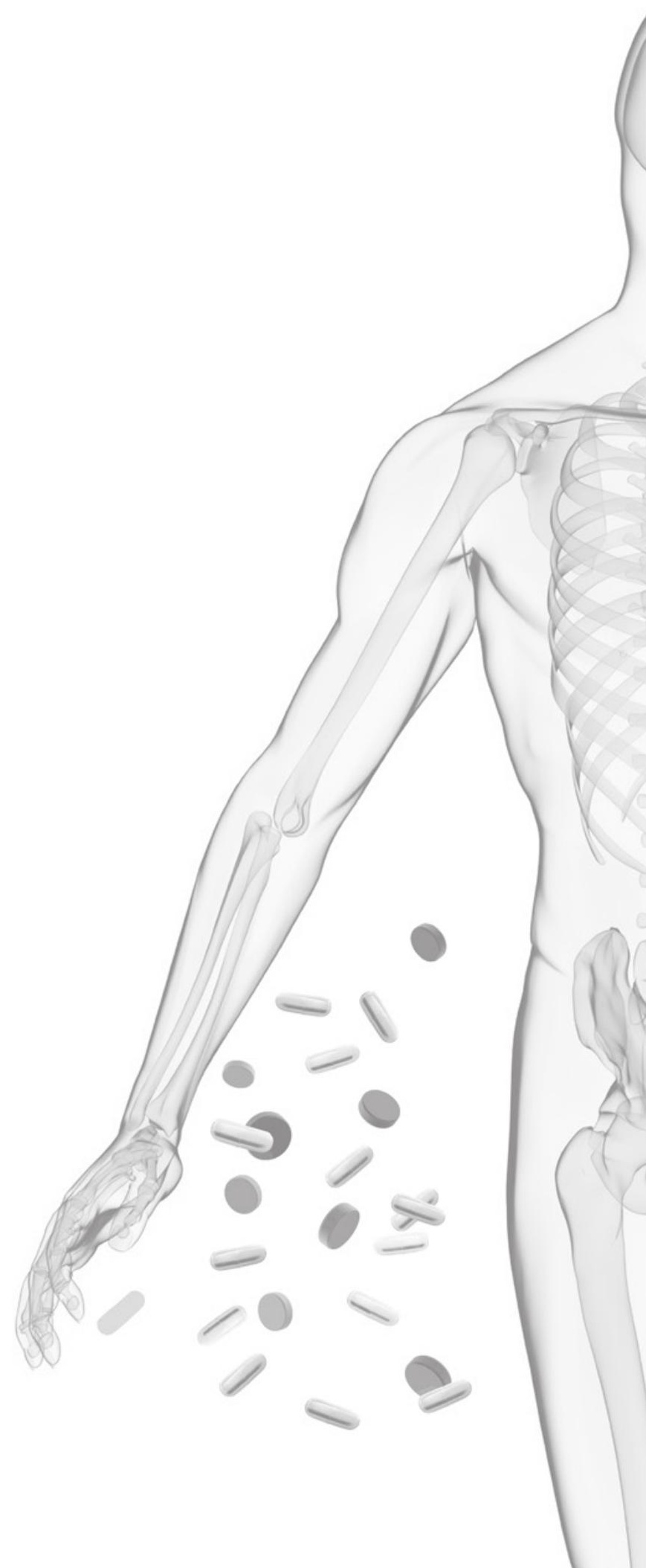




\section{CHAPTER

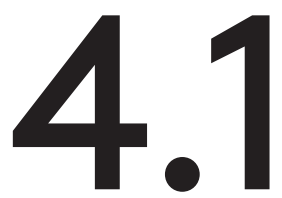

\section{STATIN USE AND MARKERS OF IMMUNITY IN THE DOETINCHEM COHORT STUDY}

H.J.I. De Jong, J.G.M.C. Damoiseaux, R.J. Vandebriel, P.C. Souverein,

E.R. Gremmer, M. Wolfs, O.H. Klungel, H. Van Loveren, JW. Cohen Tervaert, W.M.M. Verschuren 


\section{ABSTRACT}

\section{Introduction}

It has been suggested that statins can both stimulate and suppress the immune system, and thereby, may influence autoimmune diseases. Therefore, we studied effects of statins on innate and adaptive immunity, and self-tolerance by measuring serological levels of $\mathrm{C}$-reactive protein (CRP), neopterin, immunoglobulin $\mathrm{E}$ (IgE) antibodies and the presence of autoantibodies (antinuclear antibodies (ANA) and IgM rheumatoid factor (RF)) in the general population.

\section{Methods}

We conducted a nested case-control study within the population-based Doetinchem cohort. Data from health questionnaires, serological measurements and information on medication from linkage to pharmacy-dispensing records were available. We selected 332 statin users (cases) and 331 non-users (controls), matched by age, sex, date of serum collection, history of cardiovascular diseases, diabetes mellitus type II and stroke. Multivariate regression analyses were performed to estimate effect of statins on the immune system.

\section{Results}

The median level of CRP in statin users (1.28 mg/L, interquartile range (IQR): 0.59-2.79) was lower than in non-users (1.62 mg/L, IQR: 0.79-3.35), which after adjustment was estimated to be a $28 \%$ lower level. We observed an inverse association between duration of statin use and CRP levels. Elevated levels of $\operatorname{lgE}(>100 \mathrm{IU} / \mathrm{mL})$ were more prevalent in statin users compared to non-users. A trend towards increased levels of IgE antibodies in statin users was observed, whereas no associations were found between statin use and levels of neopterin or the presence of autoantibodies.

\section{Conclusions}

In this general population sub-sample, we observed an anti-inflammatory effect of statin use and a trend towards an increase of IgE levels, an surrogate marker for T helper (Th) 2 responses without a decrease in neopterin levels, a surrogate marker for Th1 response and/or self-tolerance. We postulate that the observed decreased inflammatory response during statin therapy may be important but is insufficient to induce loss of self-tolerance. 


\section{INTRODUCTION}

Numerous clinical trials have demonstrated that statins, or anti-3-hydroxy-3-methylglutaryl coenzyme A reductase (HMG-CoA or HMGCR) inhibitors, are effective in the primary and secondary prevention of cardiovascular diseases 1-3. In addition to lowering cholesterol, statins have anti-inflammatory and immunomodulatory properties which also may contribute to the beneficial effects of these drugs 4,5 . Several studies of patients with rheumatoid arthritis (RA) and experimental studies in collagen-induced arthritis suggested beneficial effects of statins in arthritis 6-12. Similarly, in studies of antiphospholipid syndrome, vasculitis, systemic sclerosis, and systemic lupus erythematosus (SLE) patients and murine lupus models, attenuation of lupus activity has been ascribed to statins ${ }^{13-20}$. On the other hand, it has been postulated that statins may facilitate the loss of self-tolerance that could potentially result in autoimmune diseases. Indeed, several case-reports and reviews have linked statin use with autoimmune disorders, such as lupus-like syndrome, vasculitis, polyand dermatomyositis, and necrotising autoimmune myositis 21-25. Furthermore, we recently demonstrated that statins are associated with an increased risk of developing RA, SLE and polymyalgia rheumatica 26-29.

The proposed mechanisms by which statins may facilitate and/or be protective for the development of autoimmune diseases are unclear. As described in various studies, statins seem to affect the functions of immune cells, including natural killer cells, monocytes, macrophages and $T$ cells ${ }^{30}$. Statins have been shown reduce pro-inflammatory cytokines, including tumor necrosis factor (TNF)-a, interleukin (IL)-6 and IL-8, and the levels of C-reactive protein (CRP), a marker of the innate immune system reflecting underlying systemic inflammation 31-35. Statins may also block stimulation of T cells and inhibit interferon (IFN)-gamma induced macrophage activation ${ }^{30}$, resulting in suppressed secretion of neopterin ${ }^{36}$. In a murine model mimicking multiple sclerosis, atorvastatin promoted the differentiation of Thelper (Th) 0 into Th2 cells, resulting in the systemic production of Th2 cytokines (IL-4, IL-5, $\mathrm{IL}-10$, and transforming growth factor beta (TGF- $\beta$ ) ${ }^{37}$. IL-4 is a cytokine that induces differentiation of naive helper T cells (Th0 cells) to Th2 cells. Upon activation by IL-4, Th2 cells subsequently produce additional IL-4 which is responsible for the induction of immunoglobulin E ( $\mathrm{gEE}$ ) synthesis by B cells. Levels of circulating IgE antibodies are representative for Th2 immune responses 38,39, whereas neopterin is considered to be a marker of Th1 responses 36 . It has been suggested that statins may induce a shift in the Th1/Th2 balance by their direct effect on T cells. A shift in the Th1/Th2 balance may dysregulate the immune homeostasis and can lead to the breakdown of self-tolerance, precipitating autoimmunity $24,37,40$. To study the relationship between statin use and different aspects of the immune system, we assessed whether statins influenced the immune responses, by measuring CRP, neopterin levels, IgE antibodies 
and some prevalent autoantibodies (IgM rheumatoid factor (RF) and antinuclear antibodies (ANA)) in subjects from the general population who were either statin user or non-user.

\section{MATERIALS AND METHODS}

\section{Study setting}

Subjects from the Dutch Doetinchem Cohort Study were linked to the PHARMO Record Linkage System (PHARMO-RLS), using information on sex, and date of birth and postal code in order to obtain information on the use of statins to study markers representative of the immune status.

The Doetinchem Cohort Study is a population-based longitudinal study among inhabitants of the Dutch town Doetinchem. The main objective of this ongoing cohort study is to investigate the impact of (changes in) lifestyle and biological risk factors on the incidence of chronic diseases. A total of 12405 men and women aged 20-59 years at baseline were examined in the years 1987-1991 (round 1) and a two-third random sample of these participants has been invited for (re-)examinations at 5-year intervals, during 1993-1997 (round 2), 1998-2002 (round 3) and 2003-2007 (round 4). Details on the Doetinchem Cohort Study have been previously described ${ }^{41}$. We used data from all four examination rounds of the Doetinchem Cohort Study. At every examination round demographic, lifestyle and health characteristics were collected using a self-administered questionnaire, including items regarding smoking and alcohol habits, educational level and physical activity. Additionally, participants underwent physical examination which included anthropometric and blood pressure measurements, and collection of blood samples. The Doetinchem Cohort Study was approved according to the guidelines of the Helsinki Declaration by the external Ethics Committee of the Dutch TNO Research Institute. Linkage between the Doetinchem Cohort Study and PHARMO-RLS has been conducted for subjects with an agreement in their informed consent 41 .

PHARMO-RLS is a population-based database, comprising pharmacy-dispensing and hospital admission data of approximately 2.3 million community-dwelling inhabitants of 48 geographically defined areas in the Netherlands from 1985 onwards. With regard to prescription drugs, pharmacy records are virtually complete since the majority of Dutch inhabitants are registered with a single community pharmacy. For this study, we used drug-dispensing and hospitalisation data. The pharmacy dispensing records include information on the type of drug dispensed, dispensing date, amount dispensed and prescribed dosage regimen. Hospitalisation data include detailed information on the primary and secondary discharge diagnoses, and dates of hospital admission and discharge. Drugs were coded according to the 
Anatomical Therapeutic Chemical (ATC) classification, and hospital diagnoses were coded according to the ICD-9-Cm codes 42,43. Within these linked datasets, each subject was followed from the date of the first prescription of statins until collection of serum during examination.

\section{Study population}

Linkage between the Doetinchem Cohort Study and PHARMO-RLS has been conducted for 9179 subjects. Of these subjects, 1779 were statin users. Clinical examination data were available for 778 statin users. For each statin user, one nonuser of statins was randomly selected and matched for age (+/- five years), sex, date of examination, and history of cardiovascular diseases, diabetes mellitus type II and stroke. After matching on these factors, 1016 subjects were included in the analysis. For the purpose of the study, subjects who used corticosteroids $(n=22)$ one month before the date of examination, or who ever used disease modifying anti-rheumatic drugs (DMARD) $(n=29)$, or had a diagnosis of an autoimmune disease before the date of examination were excluded, as were subjects who did not have a registered medical history for at least one year $(n=73)$ at entrance of the linked data from the Doetinchem Cohort Study and PHARMO-RLS. Of these 892 subjects, 229 blood samples could not be retrieved. A total of 663 subjects (332 users and 331 non-users of statins) remained for the first analysis. For the second analysis, we selected from these 663 subjects, 192 subjects for whom blood samples were available from multiple rounds of examination. The study design is depicted in Figure 1.

\section{Exposure definition}

Exposure to statins was defined as the use of at least one prescription of any approved and commercially available statin (pravastatin, simvastatin, rosuvastatin, atorvastatin, fluvastatin) in the Netherlands before each date of examination. Cerivastatin was also included in this study, although cerivastatin was withdrawn from the market in the year 2001. Cumulative duration and daily dose of statins until examination was assessed, as well as the sum of prescriptions, defined daily doses (DDD) and adherence to statins 26 . Adherence to statin use was calculated by dividing the sum of the days' supply by the total number of days between the first prescription and the last prescription of statins in the year before the date of examination, multiplied by $100 \%$. To determine adherence to statin use, we excluded subjects who received one prescription of statins, and who used no statin in the year before the date of examination. Subjects who received one prescription of statins before the date of examination were not excluded from other analyses. The potency of statins was determined by taking type and dose of statins into account, in order to control for the fact that different types and doses of statins differ with respect to the percentage reduction in low-density lipoprotein (LDL) cholesterol 44. 


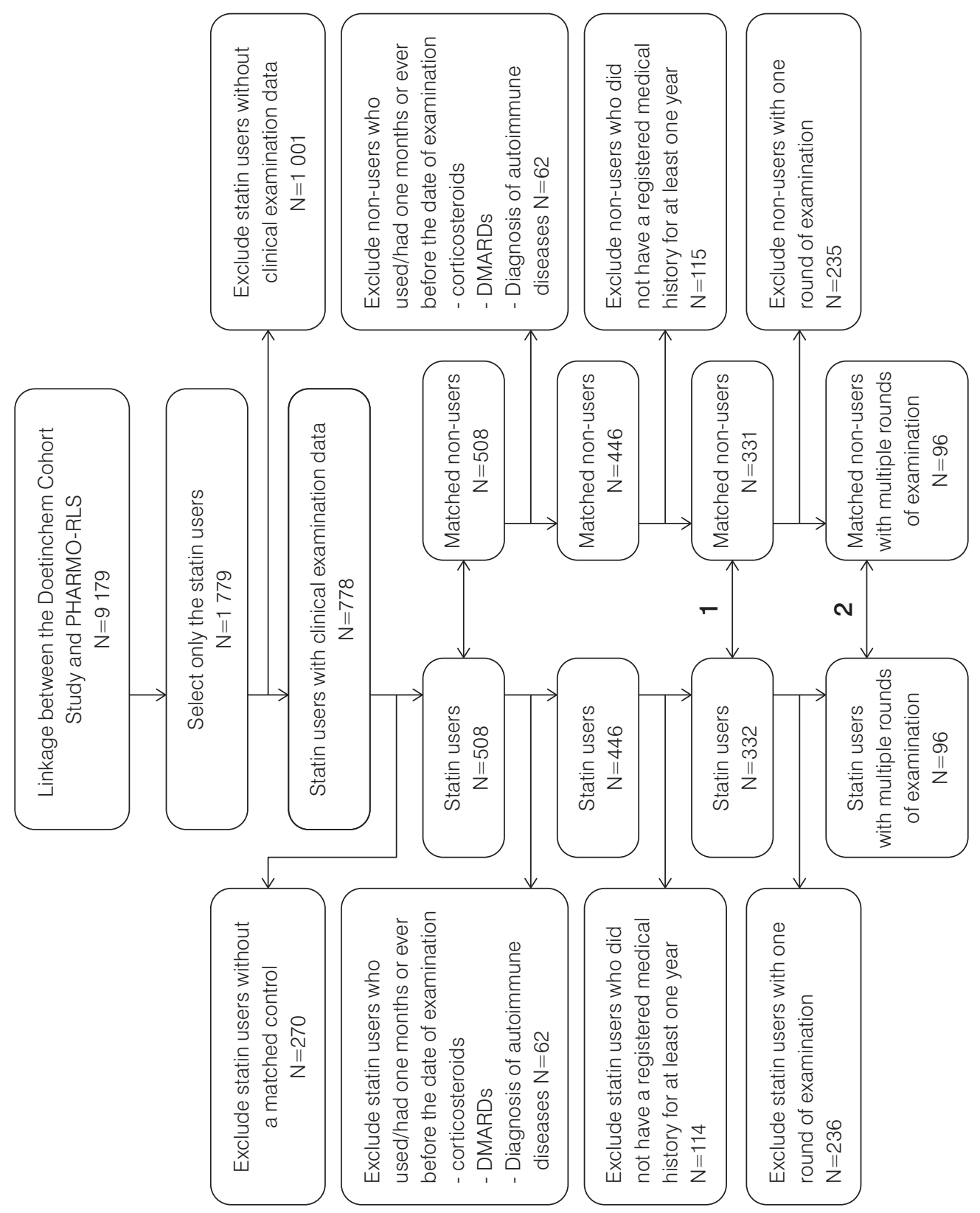

Figure 1 Study design.

1. Cross sectional study; 2. Longitudinal study. 


\section{Outcome definition}

Non-fasting venous blood samples were drawn in each examination round. Blood samples were then centrifuged and separated sera were stored in $0.5 \mathrm{~mL}$ aliquots at $-20^{\circ} \mathrm{C}$ until analyses. High sensitivity CRP was determined on a clinical autoanalyser (LX20-Pro, Beckman Coulter, Mijdrecht, the Netherlands), using standard kits. Data are expressed as $\mathrm{mg} / \mathrm{L}$. CRP levels lower than $2 \mathrm{mg} / \mathrm{L}$ are considered as normal 45 . Serum concentration of neopterin was measured by commercially available enzymelinked immunosorbent assay (ELISA) kits (IBL, Hamburg, Germany, distributed via Mediphos, Renkum, the Netherlands) according to manufacturer's instructions 46. Data are expressed as $\mathrm{ng} / \mathrm{mL}$. Neopterin levels lower than $2.5 \mathrm{ng} / \mathrm{mL}$ were considered as normal (manufacturer's recommendation). Levels of IgE antibodies were measured by using an Immunocap 250 (Phadia, Uppsala, Sweden), following manufacturer's instructions. Data are expressed as $\mathrm{IU} / \mathrm{mL}$. Total IgE levels below $100 \mathrm{IU} / \mathrm{mL}$ were considered normal 47 . Sera were diluted 1:80 with phosphate buffered saline (PBS), and were screened for ANA by indirect immunofluorescence assay on HEp-2000 cells (ImmunoConcepts, Sacramento, CA, distributed via BMD, Antwerpen, Belgium) 48 . IgM RF was determined with an ELISA (Sanquin Reagents, Amsterdam, the Netherlands), using a previously described protocol 49 . Serum levels above $12.5 \mathrm{IU} / \mathrm{mL}$ were considered positive based on measurements in a group of normal healthy blood donors in the Netherlands ( $n=665$ ), which yielded IgM RF values below $12.5 \mathrm{IU} / \mathrm{mL}$ in $96 \%$ of the cases according to manufacturer's instructions 49 . The intra-assay and interassay variability were less than $10 \%$ for all test systems. To eliminate inter- assay variability, all samples from one patient were tested in a single assay.

\section{Potential confounding variables}

The following variables were considered as potential confounders: body mass index (BMI), educational level, smoking and drinking behaviour, physical activity level, parental history of myocardial infarction (MI), levels of total cholesterol and high-density lipoprotein (HDL) cholesterol, hypertension and asthma, and the use of non-steroidal anti-inflammatory drugs (NSAIDs), corticosteroids, proton pump inhibitors (PPIs), aspirin, anti-hypertensive and anti-diabetic drugs, antibiotics, antidepressants and hormone replacement therapy in the six months prior to the date of examination 50 . Parental history of $\mathrm{MI}$ was divided into two variables: 1) father with a history of $\mathrm{Ml}$ and 2) mother with a history of $\mathrm{Ml}$, both with an age onset of $<60$ years. Hypertension was considered present when the subject had a systolic blood pressure of $140 \mathrm{mmHg}$ or higher, a diastolic blood pressure of $90 \mathrm{mmHg}$ or higher and/or used anti-hypertensive drugs. The matching factors age, sex, date of serum collection, and a history of cardiovascular diseases, diabetes mellitus type II and stroke were included in all models. In addition, all other covariables that changed the regression coefficient for statin use by $\geq 10 \%$ were entered into the model 51 . 


\section{Statistical analysis}

Baseline characteristics were reported as mean (SD) for continuous variables and proportion (percentage) for categorical variables. At baseline, comparisons between the non-users and users of statins were analysed using independent Student's t and Mann-Whitney tests for continuous variables, and Chi-square and Fisher exact tests for categorical variables. Skewed data were transformed logarithmically to approximate normal distribution. Distribution of levels of neopterin, IgE antibodies and CRP were positively skewed, and are presented as median with interquartile ranges (IQR).

The associations between statin use and CRP and neopterin levels were studied in a linear regression model with adjustment for age, sex, date of examination, history of cardiovascular diseases, diabetes mellitus type II and stroke (model 1). In the second model lifestyle factors and cardiovascular disease risk factors were added, namely smoking status, anti-hypertensive drugs and total cholesterol levels. Similarly, the association of statin use with IgE levels was analysed with additional adjustment for asthma. Log-transformed markers CRP, neopterin and IgE antibodies were back transformed for presentation and the associations are expressed as slope $(\beta)$ with confidence intervals (Cls), representing the expected percentage change in the outcome 52. Associations between statin use and the presence of elevated levels of CRP, neopterin and IgE, and ANA and IgM RF were calculated using logistic regression models, analyses were adjusted for the same variables, as listed previously. The associations are expressed as odds ratio (OR) with Cls. We evaluated effects of cumulative duration and daily dose, DDDs, number of prescriptions, adherence and potency of statins on the immune status. To test $P$ for trend of the associations across increasing quartiles of cumulative duration and daily dose, DDDs, number of prescriptions, adherence or potency of statins, the median values of these different exposure aspects of statin use were assigned to each quartile and used as a continuous variable in the linear regression model.

Because of a potential modifying effect due to the presence of cardiovascular diseases, the analysis was stratified according to history of cardiovascular diseases. Changes in the immune system with aging and sex differences have been reported. Therefore, age- ( $<50, \geq 50$ years) and sex-stratified analyses for the evaluation of effect modification were carried out 53,54 .

To study the relationship between statin use and the levels of CRP and IgE antibodies during follow-up, we used linear mixed-effects models for the analysis of repeated measurements with adjustment for the matching and baseline covariables, as described above. The model deals with the correlation between repeated measurements in a subject, and allows subjects to have unequal gaps and numbers of observations. Only subjects with at least two serological measurements were included in the model. The random effects of the model include a random intercept and/or slope of time. In the model where we only included a random intercept, specification of a random 
slope did not change the results in a relevant way. Data are presented as $\beta$ 's with Cls and denotes the adjusted percentage change in the levels of CRP and IgE antibodies compared between and within statin users and non-users. $\mathrm{P}<0.05$ was considered statistically significant using a 2-tailed test. Data were missing on several variables as listed in table 1. The missing values were imputed by the multiple imputation method by using the Markov Chain Monte Carlo Method 55. All original outcome and covariables presented in table 1 were included in the imputation model. Twenty imputation sets were created, analysed and pooled by the MIANALYZE procedure. Baseline measurements of complete and imputed cases were compared based on the means and frequencies. Results from the complete case and multiple imputation analyses were compared and multiple imputation analyses are presented. All analyses were performed using SAS version 9.2 (SAS Institute, Cary, NC).

\section{RESULTS}

\section{Baseline characteristics}

Baseline characteristics of the study population are presented in table 1. The study comprised 332 statin users of whom 196 (59\%) were men with a mean age of 59.5 (SD 8.0) years and 331 non-users of whom 194 (59\%) were men with a mean age of 59.3 (SD 8.0) years. In statin users, total cholesterol levels and BMI were higher and the level of HDL cholesterol was lower than in non-users. Hypertension and cardiovascular disease were more frequently reported among statin users than non-users. As a result of these differences, anti-hypertensive drugs, aspirin and PPIs were more used among statin users.

\section{Inflammation}

Measurements of immune parameters are presented in Table 2. The median (IQR) level of CRP was significantly lower for statin users, $1.28(0.59-2.79) \mathrm{mg} / \mathrm{L}$, than for non-users $1.62(0.79$ - 3.35) mg/L. After controlling for the matching variables, antihypertensive drugs, total cholesterol levels and smoking status, a lower CRP level was observed in statin users compared with non-users (difference of -28\% for statin use vs. non-use, $P<0.01$ ). When we used a cut-off value of less than $2 \mathrm{mg} / \mathrm{L}$ to discriminate between normal and increased levels of CRP, statin use was associated with the presence of low levels of CRP (OR: 0.67; 95\% Cl: 0.45 to 0.99).

\section{Immunomodulation}

We found no difference in levels of neopterin or the prevalence of low levels of neopterin $(<2.5 \mathrm{ng} / \mathrm{mL})$ between statin users and non-users. The median IgE level in statin users was $28.6 \mathrm{IU} / \mathrm{mL}$ compared to $25.3 \mathrm{IU} / \mathrm{mL}$ in non-users. The prevalence of 
Table 1 Baseline characteristics of the study population

\begin{tabular}{|c|c|c|c|c|c|}
\hline & $\begin{array}{l}\text { Statin } \\
\text { users } \\
\mathrm{N}\end{array}$ & $\begin{array}{l}\text { Statin } \\
\text { users } \\
(n=332)\end{array}$ & $\begin{array}{l}\text { Non- } \\
\text { users } \\
\mathrm{N}\end{array}$ & $\begin{array}{l}\text { Non- } \\
\text { users } \\
(n=331)\end{array}$ & $p$ value \\
\hline Men, \% & 332 & $196(59)$ & 331 & $194(59)$ & - \\
\hline Mean age in years (SD) & 332 & $59.5 \pm 8.0$ & 331 & $59.3 \pm 8.0$ & - \\
\hline Mean $\mathrm{BMI}$ in $\mathrm{kg} / \mathrm{m}^{2}(\mathrm{SD})^{*}$ & 316 & $26.8 \pm 3.4$ & 292 & $26.1 \pm 3.6$ & 0.02 \\
\hline Low education level, \% & 253 & $136(54)$ & 236 & $131(56)$ & 0.72 \\
\hline History myocardial infarction father, \% & 316 & $41(13)$ & 292 & $26(9)$ & 0.11 \\
\hline Current smoking, $\%$ & 252 & $74(29)$ & 236 & $57(24)$ & 0.10 \\
\hline Regular alcohol consumption, \% & 299 & $193(65)$ & 271 & $186(69)$ & 0.61 \\
\hline Physical active, \% & 236 & $188(80)$ & 216 & $156(72)$ & 0.06 \\
\hline Mean total cholesterol in mmol/L (SD) & 316 & $6.82 \pm 1.18$ & 291 & $5.68 \pm 1.01$ & $<0.0001$ \\
\hline Mean HDL cholesterol in $\mathrm{mmol} / \mathrm{L}(\mathrm{SD})^{\dagger}$ & 316 & $1.19 \pm 0.32$ & 291 & $1.30 \pm 0.35$ & $<0.0001$ \\
\hline \multicolumn{6}{|l|}{$\begin{array}{l}\text { Disease history before date } \\
\text { of examination, \% }\end{array}$} \\
\hline Diabetes & 266 & $67(25)$ & 249 & $50(20)$ & - \\
\hline Hypertension & 332 & $147(47)$ & 331 & 99 (34) & 0.002 \\
\hline Cardiovascular disease & 332 & $87(26)$ & 331 & $25(8)$ & $<0.0001$ \\
\hline Stroke & 315 & $11(3)$ & 290 & $9(3)$ & - \\
\hline Asthma & 261 & $5(2)$ & 245 & $10(4)$ & 0.15 \\
\hline Cancer & 332 & $16(5)$ & 331 & $15(5)$ & 0.86 \\
\hline \multicolumn{6}{|l|}{$\begin{array}{l}\text { Drug use six months before date } \\
\text { of examination (\%) }\end{array}$} \\
\hline Corticosteroids & 332 & $3(1)$ & 331 & $3(1)$ & 1.00 \\
\hline NSAIDs ${ }^{\ddagger}$ & 332 & $44(13)$ & 331 & $53(16)$ & 0.31 \\
\hline Fibrates & 332 & $1(0)$ & 331 & $0(0)$ & 1.00 \\
\hline Anti-hypertensive drugs & 332 & $135(41)$ & 331 & $53(16)$ & $<0.0001$ \\
\hline Anti-diabetic drugs & 332 & $29(9)$ & 331 & $23(7)$ & 0.39 \\
\hline Aspirin & 332 & $78(24)$ & 331 & $15(5)$ & $<0.0001$ \\
\hline HRT§ & 332 & $12(4)$ & 331 & $10(3)$ & 0.67 \\
\hline Antibiotics & 332 & $32(10)$ & 331 & $26(8)$ & 0.42 \\
\hline Antidepressants & 332 & $17(5)$ & 331 & $18(5)$ & 0.85 \\
\hline PPIs"l & 332 & $26(8)$ & 331 & $14(4)$ & 0.05 \\
\hline $\begin{array}{l}\text { *BMI, Body mass index } \\
\text { † HDL, High-density lipoprotein } \\
\text { ₹ NSAIDs, Nonsteroidal anti-inflammatory d } \\
\text { § HRT, Hormone replacement therapy } \\
\text { " PPIs, Proton pump inhibitors }\end{array}$ & ugs & & & & \\
\hline
\end{tabular}

elevated levels of $\mathrm{IgE}$ antibodies $(>100 \mathrm{IU} / \mathrm{mL}$ ) was higher in statin users compared to non-users. A trend towards higher levels of IgE antibodies was observed in statin users compared with non-users, although this difference did not meet the criteria for significance. 


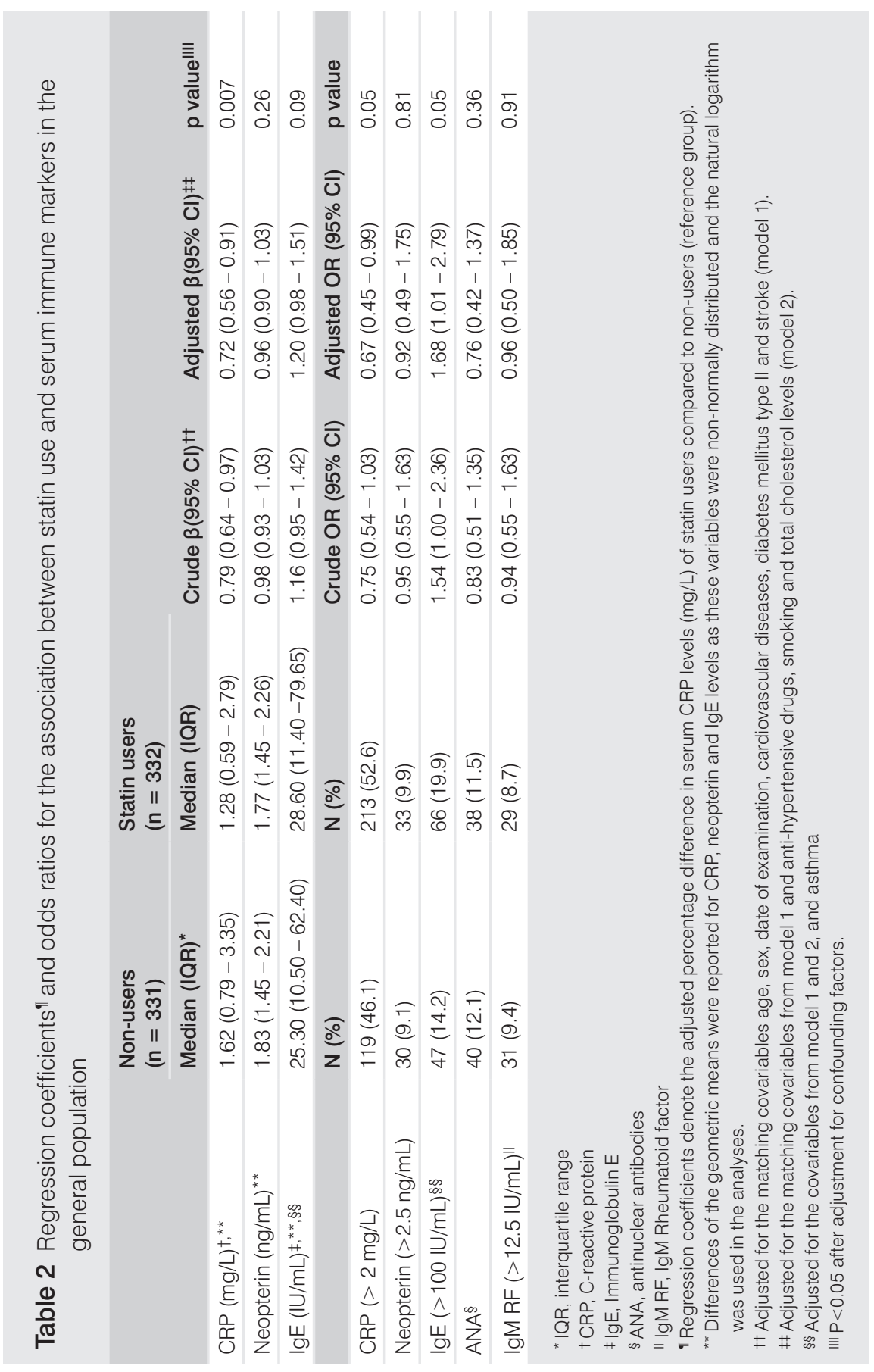




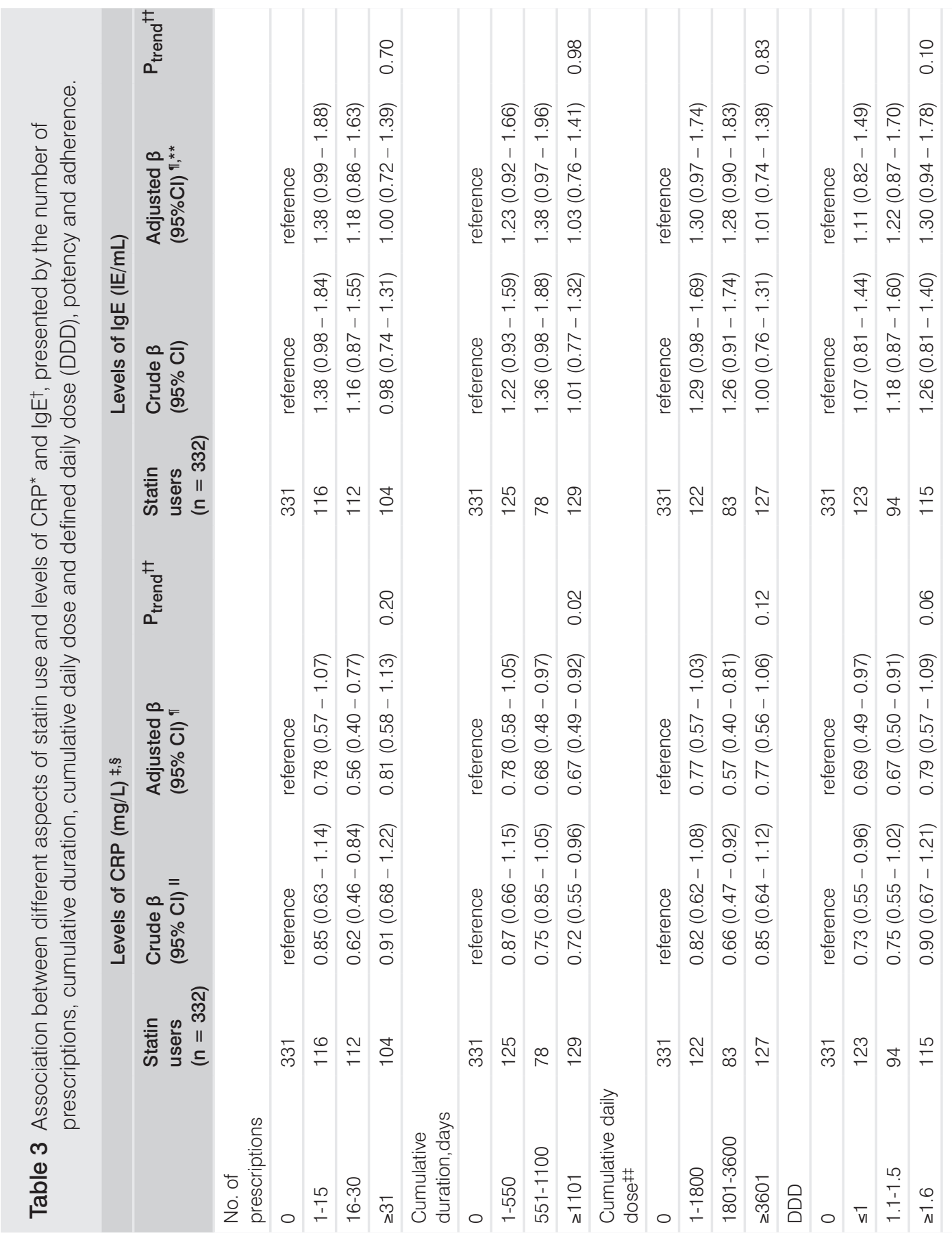




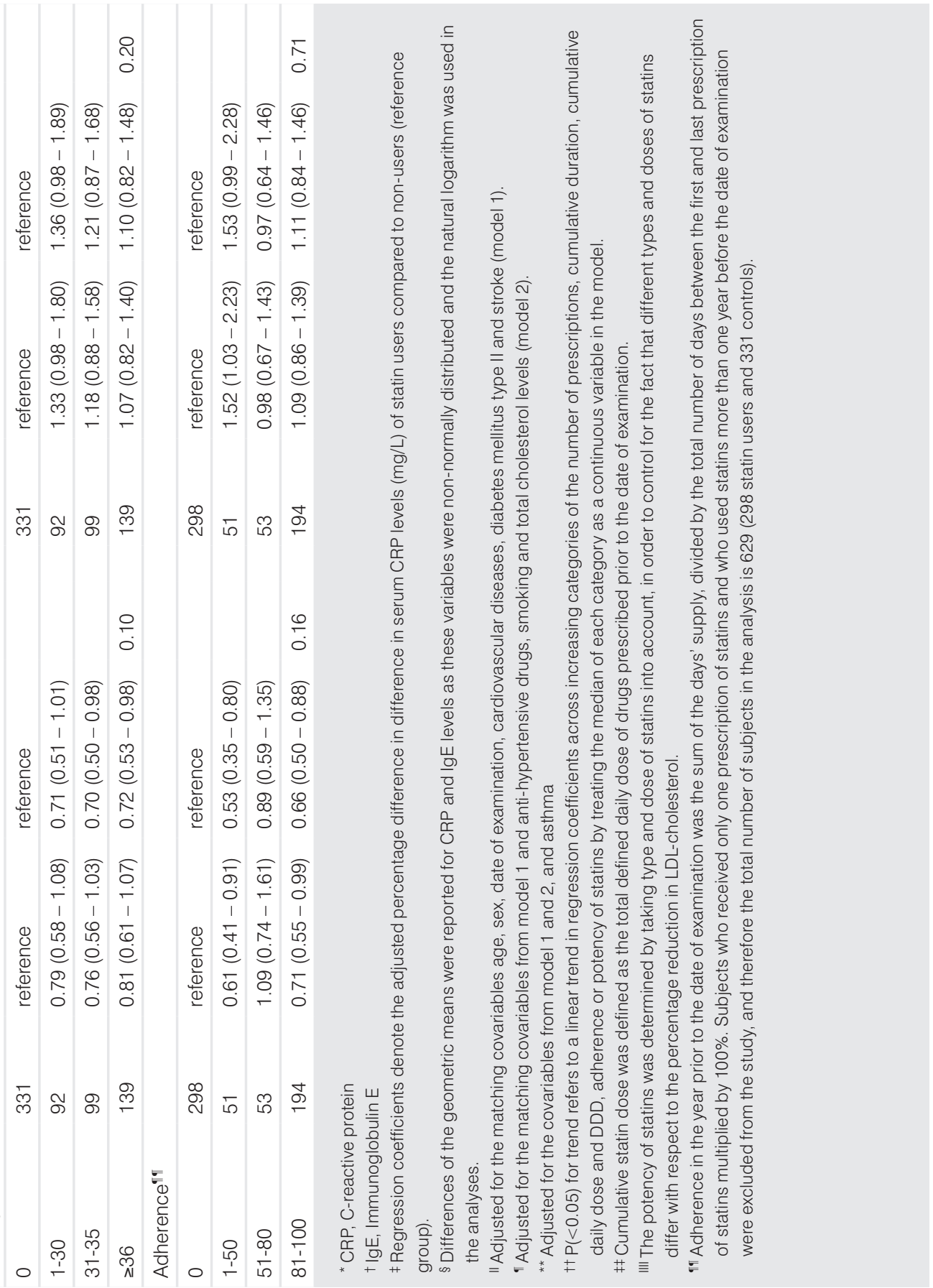




\section{Self-tolerance}

No associations were observed between statin use and the presence of the autoantibodies, ANA and IgM RF.

\section{CRP and IgE levels and different aspects of exposure to statins}

As we observed associations between statin use and levels of CRP and IgE antibodies, we studied these associations in detail in statin users by the different aspects of exposure to statins. We present in table 3 the regression coefficients for the different aspects of exposure to statins in relation to CRP and lgE levels, adjusted for confounders. Regarding the number of prescriptions, cumulative duration and daily dose, DDDs, potency and adherence to statins, associations with CRP levels were inverse, although none of them were statistically significant. In addition, an association between increasing number of days of statin use and decreased levels of CRP was observed ( $\left.P_{\text {trend }}=0.02\right)$. For IgE levels, the fully adjusted associations with different aspects of exposure to statins were positive, but no association was statistically significant.

\section{CRP and IgE levels and different groups of age, sex and history of cardiovascular diseases}

The associations between statin use and levels of CRP did not differ between strata of sex, age and history of cardiovascular diseases (data not shown). However, levels of IgE antibodies were higher in statin users versus non-users who were older than 60 years and had a history of cardiovascular disease (data not shown).

\section{Development of CRP and IgE levels over time}

Cross-sectional analyses did not show differences in the levels of neopterin, and the presence of ANA and IgM RF between statin users and non-users. Therefore, we only studied the change in CRP and IgE levels over time between statin users and non-users (table 4). After controlling for the matching variables, anti-hypertensive drugs, total cholesterol levels and smoking status, the change over time in mean CRP level was lower in statin users compared to non-users, by on average 29\% $(p=0.04)$. No difference in the change over time in mean levels of lgE antibodies between statin users and non-users was observed. We found no effect modification between time and statin treatment between users and non-users of statins, indicating that the difference in CRP levels remained constant over time. 
Table 4 Regression coefficients ${ }^{\ddagger}$ for the change in $\mathrm{CRP}^{*}$ and $\lg \mathrm{E}^{\dagger}$ levels over time between statin users and non-users.

\begin{tabular}{llll} 
& Crude $\beta(95 \% \mathrm{Cl})$ §,II & Adjusted $\beta(95 \% \mathrm{Cl})^{\text {ฯ }}$ & p value $^{\dagger \dagger}$ \\
$\mathrm{CRP}(\mathrm{mg} / \mathrm{l})^{\neq \neq}$ & $0.81(0.98-1.07)$ & $0.71(0.51-0.98)$ & 0.04 \\
\hline $\operatorname{lgE}(\mathrm{IE} / \mathrm{ml})^{* *}$ & $0.87(0.84-1.41)$ & $0.97(0.84-1.13)$ & 0.70
\end{tabular}

* CRP, C-reactive protein

+ IgE, Immunoglobulin $\mathrm{E}$

₹ Regression coefficients denote the adjusted percentage change in the levels of CRP and IgE antibodies compared between and within statin users and non-users (reference group).

$\S$ Differences of the geometric means were reported for CRP and IgE levels as these variables were non-normally distributed and the natural logarithm was used in the analyses.

" Adjusted for the matching covariables age, sex, date of examination, cardiovascular diseases, diabetes mellitus type II and stroke (model 1).

^ Adjusted for the matching covariables from model 1 and anti-hypertensive drugs, smoking and total cholesterol levels (model 2).

** Adjusted for the covariables from model 1 and 2, and asthma

t† $\mathrm{P}<0.05$ after adjustment for confounding factors.

邦 The random-effects portion of the model consists only of a random intercept.

\section{DISCUSSION}

In the present study, we observed that statins suppress the innate immune response, by decreasing the levels of CRP, both cross-sectionally and over time. A more detailed analysis on different aspects of exposure to statins, showed an inverse association between duration of statin use and levels of CRP. Furthermore, we observed a trend towards higher levels of IgE antibodies in statin users, which infers that it did not meet the pre-specified criteria for significance. Neopterin levels were not affected. Finally, ANA and IgM RF were not different between statin users and non-users suggesting that the use of statins was not associated with a loss of self-tolerance.

Importantly, our study consistently showed that the use of statins is associated with decreased levels of CRP in the general population, as previously described in population-based studies and clinical trials $33,56-60$. Essentially, by confirming this well-known association between statin use and decreased levels of CRP, it demonstrates that we have used appropriate methods for patient selection. This strengthens the conclusions of our study.

Apart from the anti-inflammatory effects, it has been suggested that statins may have a direct immunomodulating effect on T cells and may promote a shift from Th1 to Th2 immune responses, possibly leading to a dysregulation in the immune homeostasis 24,30,37. Cherfan et al. reported that statins did not affect Th1 cells but suppressed Th2 
responses ${ }^{58}$. In contrast, other studies suggested that statins promote a Th2 bias 61,62. In our study, we observed a trend towards increased levels of IgE antibodies, representative of Th2 responses, but this was not accompanied by a decrease in neopterin levels, a marker for Th1 cell activity. This is in agreement with two other studies showing that treatment with statins had no effect on the levels of neopterin 63,64. However, two studies including patients with cardiovascular diseases showed that neopterin levels declined during statin treatment 36,65 , indicating a suppressive effect on Th1 cells. We found no association between statin use and the presence of the most prevalent autoantibodies, ANA and IgM RF 66,67, suggesting maintenance of self-tolerance. So far, an indication for an association between statin use and the presence of ANA was shown in case reports of lupus-like syndrome, poly- and dermatomyositis 24 . To date, no study has been performed on statin use and the presence of RF in the general population. The study of Chodick et al. actually showed a decreased risk of RA in persistent statin users ${ }^{7}$. In this study, persistent statin users were compared to non-persistent statin users and were followed until the study outcome (RA). In our study, we compared statin users with non-users. The outcome variable autoimmune disease, i.e., RA, was not part of our study. We measured incidence of IgM RF, in combination with ANA, merely as markers for loss of selftolerance. However, when the risk of RA was compared between statin users and non-users, three population-based studies demonstrated no association between statin use and the risk of developing RA 9,68,69.

Recently, Mammen et al. identified the presence of anti-HMGCR antibodies in statin-associated autoimmune myopathy 70 . The same authors screened a large population of statin exposed patients without myopathy and found no anti-HMGCR antibodies in these subjects ${ }^{71}$. As our observations showed that statins were not related to the development of autoantibodies in the general population, we hypothesise that statins do not themselves cause autoimmunity but that they may promote a pre-existing autoimmune-prone condition to progress towards clinically manifest diseases. Several studies demonstrated that statins may exacerbate or trigger cellular apoptosis 24,72,73, which may lead to increased levels of apoptotic cells and blebs, resulting in increased amounts of autoantigens in the circulation 74,75 . As patients treated with statins may be less efficient in clearing apoptotic cells, they may be more susceptible to autoimmune diseases. Furthermore, statins may be able to amplify autoimmunity in genetically susceptible individuals, thereby increasing the risk of developing autoimmune diseases ${ }^{76}$. It is known that statins not only interfere with the mevalonate or cholesterol synthesis pathway but also inhibit the prenylation of Rho GTPases, including Rac-1 77,78. Rac-1 is directly implicated in the activation of caspase-1 79 , which is crucial for processing and secretion of the proinflammatory cytokines IL-1 $\beta$ and IL-18, promoting autoimmunity 80,81. However, patients with mutations in the mevalonate kinase gene (e.g. hyper-lgD syndrome) may respond 
differently to the treatment with statins 82 , although the prevalence rate of this immune disorder is very low. To prove our hypothesis, a much larger cohort study (probably more than 40000 subjects ${ }^{83}$ ) should be performed.

One of the strengths of this study is the linkage between a large population-based cohort study and a registry of pharmacy and hospital discharge records, enabling us to assess data on statin use and sera from subjects in Doetinchem. Another strong feature of our study is the prospective and longitudinal design with a relatively long follow-up (>15y). Moreover, as sera were collected at several time points, our subjects were compared not only cross-sectionally but also longitudinally. Using a computerised database, PHARMO-RLS, we were allowed to use routinely recorded dispensing data from pharmacies. Consequently, recall and non-response bias were minimised. Furthermore, detailed information on confounders, including smoking status was available.

Some limitations of our study should be acknowledged. First, we selected a restricted number of immune markers in our study as statins may have influenced other T-cell-mediated responses. It has been suggested that statins block the differentiation of the pro-inflammatory Th17 cells and have a direct effect on regulatory T cells (Treg) in atherosclerotic plaques in mice and peripheral circulation of coronary patients $84-86$. Regrettably, to date, no valid markers for detecting Th17 and Treg cells in human sera are available. Furthermore, the long-term storage of our samples limited us in the selection of immune markers as our samples were stored from 2 up to 20 years (depending on the round of examination). Long-term storage of the sera samples may have affected the levels of various immune markers, such as antibodies, chemokines, cytokines and other soluble markers of immune activation. However, several studies have shown that prolonged storage did not affect CRP 87, neopterin 88 and IgE antibodies 89 . Since statin users and non-users were matched on date of examination in order to maintain an equally distributed duration of sample storage, we do not expect that long-term storage influenced our associations between statin use and the levels of CRP, neopterin and IgE antibodies. Second, despite our best efforts, matching on cardiovascular diseases was only partially achieved as the majority of the subjects with cardiovascular diseases were treated with statins. However, when we stratified for cardiovascular diseases, no differences in the association between statin use and immune markers were observed. Third, statin users or non-users may have visited a pharmacy, not participating in our computerised database to collect their prescription, possibly resulting in an underestimation of the actual statin use. However, a recent study showed that the majority of Dutch patients are loyal to their pharmacy, i.e., approximately only $11 \%$ of patients visited two or more pharmacies in 2001 90. When patients visited another pharmacy, it would most likely be a pharmacy in the same region. Since we had data from all pharmacies in the Doetinchem region, we could assess the complete medication history from a single 
statin user or non-user across these pharmacies. Therefore, we do not expect that this would have affected our findings.

Fourth, the linkage of the Doetinchem Cohort Study and the PHARMO-RLS database yielded 663 subjects who had at least one serum sample in one of the examination rounds. This restricted number of subjects may have raised concerns about the generalisability of our findings. It is conceivable that this may have affected the estimates of the baseline characteristics, but we believe to a lesser extent on the magnitude of the associations between statin use and the serological markers of (auto) immunity. Finally, it should be acknowledged that this is an observational study which may have been subject to residual confounding due to potential unmeasured differences in cardiovascular risk profile and subjects' characteristics between statin users and non-users. However, we have matched the statin users and non-users on several factors; we believe we have reduced residual confounding as much as possible.

\section{CONCLUSIONS}

In the present study, we have demonstrated that statins exert anti-inflammatory effects as shown by a decrease in levels of CRP. However, no effects on the levels of neopterin and the presence of the autoantibodies, ANA and RF, were shown. These findings suggest that this population-based study does not provide evidence that statins exert immunomodulatory effects on a Th1 response and/or loss of selftolerance. An immunomodulatory effect on Th2 response, however, cannot be excluded as we observed a slight increase in IgE levels. Further research is warranted to investigate the immunomodulatory effects of statins in peripheral blood of healthy individuals from a large population-based cohort study.

\section{Key points}

Statins suppress the innate immune response, by decreasing the levels of CRP. Statins do not exert immunomodulatory effects on Th1 response and/or loss of self-tolerance.

An immunomodulatory effect on Th2 response, however, cannot be excluded.

\section{Acknowledgements}

The authors thank the epidemiologists and field workers of the Municipal Health Service in Doetinchem for their contribution to the data collection. The data-managers A. Blokstra, J. van der Laan and R J. de Klein of the Centre for Nutrition, Prevention and Health Services from the National Institute for Public Health are acknowledged for providing the data for the present study. S. Belitser from the Utrecht University is gratefully acknowledged for her expert advice in statistics. Furthermore, the authors 
thank L. de la Fonteyne, A. de Klerk, B. Nagarajah H. Verharen, B. Verlaan, J. Vermeulen, and Dr I. Tonk for collecting the samples from the freezers and E. Jansen for conducting the hs-CRP measurements at the Centre for Health Protection Research from the National Institute for Public Health and the Environment. In addition, we would like to thank the technicians J. Austen, J. Barron-Houben, Y. Knapen-Portz, J. Knops-Janssen, C. Langejans, K. Mallet, J. Niessen, D. van Opbergen, M-P Paulissen and C. Wieczorek from the Central Diagnostic Laboratory, Maastricht University Medical Centre for their assistance in measuring the samples. 


\section{REFERENCES}

1. Baigent C, Keech A, Kearney PM, et al. Efficacy and safety of cholesterol-lowering treatment: prospective meta-analysis of data from 90,056 participants in 14 randomised trials of statins. Lancet 2005; 366(9493): 1267-78.

2. Bellosta S, Paoletti R, Corsini A. Safety of statins: focus on clinical pharmacokinetics and drug interactions. Circulation 2004; 109(23 Suppl 1): III50-7.

3. Sever PS, Dahlof B, Poulter NR, et al. Prevention of coronary and stroke events with atorvastatin in hypertensive patients who have average or lower-than-average cholesterol concentrations, in the Anglo-Scandinavian Cardiac Outcomes Trial--Lipid Lowering Arm (ASCOT-LLA): a multicentre randomised controlled trial. Lancet 2003; 361(9364): 1149-58.

4. Kwak BR, Mulhaupt F, Mach F. Atherosclerosis: anti-inflammatory and immunomodulatory activities of statins. Autoimmun Rev 2003; 2(6): 332-8.

5. Arnaud C, Braunersreuther V, Mach F. Toward immunomodulatory and anti-inflammatory properties of statins. Trends Cardiovasc Med 2005; 15(6): 202-6.

6. Barsante MM, Roffê E, Yokoro CM, et al. Anti-inflammatory and analgesic effects of atorvastatin in a rat model of adjuvant-induced arthritis. Eur J Pharmacol 2005; 516(3): 282-9.

7. Chodick G, Amital H, Shalem Y, Kokia E, Heymann AD, Porath A, Shalev V. Persistence with statins and onset of rheumatoid arthritis: a population-based cohort study. PLoS Med 2010; 7(9): e1000336.

8. Funk JL, Chen J, Downey KJ, Clark RA. Bone protective effect of simvastatin in experimental arthritis. J Rheumatol 2008; 35(6): 1083-91.

9. Jick SS, Choi H, Li L, Mclnnes IB, Sattar N. Hyperlipidaemia, statin use and the risk of developing rheumatoid arthritis. Ann Rheum Dis 2009; 68(4): 546-51.

10. Leung BP, Sattar N, Crilly A, et al. A novel anti-inflammatory role for simvastatin in inflammatory arthritis. J Immunol 2003; 170(3): 1524-30

11. McCarey DW, Mclnnes IB, Madhok R, et al. Trial of Atorvastatin in Rheumatoid Arthritis (TARA): double-blind, randomised placebo-controlled trial. Lancet 2004; 363(9426): 2015-21.

12. Yamagata T, Kinoshita K, Nozaki Y, Sugiyama M, Ikoma S, Funauchi M. Effects of pravastatin in murine collagen-induced arthritis. Rheumatol Int 2007; 27(7): 631-9.

13. Scoble T, Wijetilleka S, Khamashta MA. Management of refractory anti-phospholipid syndrome. Autoimmun Rev 2011; 10(11): 669-73.

14. Shimazu H, Kinoshita K, Hino S, et al. Effect of combining ACE inhibitor and statin in lupus-prone mice. Clin Immunol 2010; 136(2): 188-96.

15. Kotyla P, Sliwinska-Kotyla B, Kucharz E. Tumor necrosis factor-alpha as a potential target in the treatment of systemic lupus erythematosus: a role for the HMG-CoA reductase inhibitor simvastatin. J Rheumatol 2006; 33(11): 2361-3.

16. Kotyla PJ. Comment on: Atorvastatin therapy improves endothelial-dependent vasodilation in patients with systemic lupus erythematosus: an 8 week controlled trial. Rheumatology 2008; 47(3): 381-2.

17. Lawman S, Mauri C, Jury E, Cook HT, Ehrenstein M. Atorvastatin inhibits autoreactive B cell activation and delays lupus development in New Zealand black/white F1 mice. J Immunol 2004; 173(12): 7641-6.

18. Ferreira GA, Navarro TP, Telles RW, Andrade LEC, Sato El. Atorvastatin therapy improves endothelial-dependent vasodilation in patients with systemic lupus erythematosus: an 8 weeks controlled trial. Rheumatology 2007; 46(10): 1560-5.

19. Abud-Mendoza C, De La Fuente H, Cuevas-Orta E, Baranda L, Cruz-Rizo J, González-Amaro R. Therapy with statins in patients with refractory rheumatic diseases: a preliminary study. Lupus 2003; 12(8): 607-11.

20. Khattri S, Zandman-Goddard G. Statins and autoimmunity. Immunol Res 2013; 56(2-3): 348-57.

21. Kay J, Finn D, Stone J. Case records of the Massachusetts General Hospital. Case 4-2006. A 79-year-old woman with myalgias, fatigue, and shortness of breath. N Engl J Med 2006; 354(6): 623-30.

22. Fauchais A, Iba Ba J, Maurage P, et al. [Polymyositis induced or associated with lipid-lowering drugs: five cases]. Rev Méd Interne 2004; 25(4): 294-8.

23. Dalakas MC. Pathogenesis and therapies of immune-mediated myopathies. Autoimmun Rev 2012; 11(3): 203-6. 
24. Noël B. Lupus erythematosus and other autoimmune diseases related to statin therapy: a systematic review. J Eur Acad Dermatol Venereol 2007; 21(1): 17-24.

25. Sen D, Rosenstein E, Kramer N. ANCA-positive vasculitis associated with simvastatin/ezetimibe: expanding the spectrum of statin-induced autoimmunity? Int J Rheum Dis 2010; 13(3): e29-31.

26. De Jong HJ, Klungel OH, Van Dijk L, et al. Use of statins is associated with an increased risk of rheumatoid arthritis. Ann Rheum Dis 2012; 71(5): 648-54.

27. De Jong HJ, Saldi SR, Klungel OH, et al. Statin-Associated Polymyalgia Rheumatica. An Analysis Using WHO Global Individual Case Safety Database: A Case/Non-Case Approach. PLoS One 2012; 7(7): e41289.

28. De Jong HJ, Tervaert JW, Saldi SR, et al. Association between statin use and lupus-like syndrome using spontaneous reports. Semin Arthritis Rheum 2011; 41(3): 373-81.

29. Vandebriel RJ, De Jong HJ, Gremmer ER, et al. Statins accelerate the onset of collagen type II-induced arthritis in mice. Arthritis Res Ther 2012; 14(2): R90.

30. Kwak B, Mulhaupt F, Myit S, Mach F. Statins as a newly recognized type of immunomodulator. Nat Med 2000; 6(12): 1399-402.

31. Nissen S, Tuzcu EM, Schoenhagen P, et al. Statin therapy, LDL cholesterol, C-reactive protein, and coronary artery disease. N Engl J Med 2005; 352(1): 29-38.

32. Ridker P, Cannon C, Morrow D, et al. C-reactive protein levels and outcomes after statin therapy. N Engl J Med 2005; 352(1): 20-8.

33. Peters SAE, Palmer MK, Grobbee DE, Crouse JR, O'Leary DH, Raichlen JS, Bots ML. C-reactive protein lowering with rosuvastatin in the METEOR study. J Intern Med 2010; 268(2): 155-61.

34. Kagami S, Kanari $\mathrm{H}$, Suto A, et al. HMG-CoA reductase inhibitor simvastatin inhibits proinflammatory cytokine production from murine mast cells. Int Arch Allergy Immunol 2008; 146 (Suppl 1): 61-6.

35. Yokota K, Miyazaki T, Hirano M, Akiyama Y, Mimura T. Simvastatin inhibits production of interleukin 6 (IL-6) and IL-8 and cell proliferation induced by tumor necrosis factor-alpha in fibroblast-like synoviocytes from patients with rheumatoid arthritis. J Rheumatol 2006; 33(3): 463-71.

36. Walter RB, Fuchs D, Weiss G, Walter TR, Reinhart WH. HMG-CoA reductase inhibitors are associated with decreased serum neopterin levels in stable coronary artery disease. Clin Chem Lab Med 2003; 41(10): 1314-9

37. Youssef S, Stüve O, Patarroyo JC, et al. The HMG-CoA reductase inhibitor, atorvastatin, promotes a Th2 bias and reverses paralysis in central nervous system autoimmune disease. Nature 2002; 420(6911): 78-84.

38. Lordan J, Bucchieri F, Richter A, et al. Cooperative effects of Th2 cytokines and allergen on normal and asthmatic bronchial epithelial cells. J Immunol 2002; 169(1): 407-14

39. Brummer E, Hanson LH, Stevens DA. IL-4, IgE, and interferon-gamma production in pulmonary blastomycosis: comparison in mice untreated, immunized, or treated with an antifungal ( $\mathrm{SCH} 39304)$. Cell Immunol 1993; 149(2): 258-67.

40. Gurevich V, Shovman O, Slutzky L, Meroni P, Shoenfeld Y. Statins and autoimmune diseases. Autoimmun Rev 2005; 4(3): 123-9.

41. Verschuren WMM, Blokstra A, Picavet HSJ, Smit HA. Cohort profile: the Doetinchem Cohort Study. Int J Epidemiol 2008; 37(6): 1236-41.

42. Herings RM, Bakker A, Stricker BH, Nap G. Pharmaco-morbidity linkage: a feasibility study comparing morbidity in two pharmacy based exposure cohorts. J Epidemiol Community Health 1992; 46(2): 136-40.

43. PHARMO. Institute for drug outcome research PHARMO records linkage system. 2011: Available at: http://www.pharmo.nl. Accessed November 13, 2011

44. Law MR, Wald NJ, Rudnicka AR. Quantifying effect of statins on low density lipoprotein cholesterol, ischaemic heart disease, and stroke: systematic review and meta-analysis. BMJ 2003; 326(7404): 1423.

45. Pearson TA, Mensah GA, Alexander RW, et al. Markers of inflammation and cardiovascular disease: application to clinical and public health practice: A statement for healthcare professionals from the Centers for Disease Control and Prevention and the American Heart Association. Circulation 2003; 107(3): 499-511.

46. Thewissen M, Damoiseaux J, Duijvestijn A, et al. Abdominal fat mass is associated with adaptive immune activation: the CODAM Study. Obesity 2011; 19(8): 1690-8 
47. Matta GME, Battaglio S, Dibello C, et al. Polyclonal immunoglobulin E levels are correlated with hemoglobin values and overall survival in patients with multiple myeloma. Clin Cancer Res 2007; 13(18): 5348-54.

48. Damoiseaux JG, Tervaert JW. From ANA to ENA: how to proceed? Autoimmun Rev 2006; 5(1): 10-7.

49. Tervaert JWC, Van Paassen P, Damoiseaux J. Type II cryoglobulinemia is not associated with hepatitis $\mathrm{C}$ infection: the Dutch experience. Ann N Y Acad Sci 2007; 1107: 251-8.

50. Dedeoglu F. Drug-induced autoimmunity. Curr Opin Rheumatol 2009; 21(5): 547-51.

51. Greenland S, Mickey RM. Re: "The impact of confounder selection criteria on effect estimation. Am J Epidemiol 1989; 130(5): 1066

52. Cole TJ. Sympercents: symmetric percentage differences on the $100 \mathrm{log}(\mathrm{e})$ scale simplify the presentation of log transformed data. Stat Med 2000; 19(22): 3109-25.

53. Schuurs $\mathrm{AH}$, Verheul $\mathrm{HA}$. Effects of gender and sex steroids on the immune response. J Steroid Biochem 1990; 35(2): 157-72.

54. Hodes RJ. Aging and the immune system. Immunol Rev 1997; 160: 5-8.

55. Sterne JA, White IR, Carlin JB, et al. Multiple imputation for missing data in epidemiological and clinical research: potential and pitfalls. BMJ 2009; 338: b2393.

56. Yoon SS, Dillon CF, Carroll M, Illoh K, Ostchega Y. Effects of statins on serum inflammatory markers: the U.S. National Health and Nutrition Examination Survey 1999-2004. J Atheroscler Thromb 2010; 17(11): 1176-82.

57. Lyngdoh T, Vollenweider P, Waeber G, Marques-Vidal P. Association of statins with inflammatory cytokines: a population-based Colaus study. Atherosclerosis 2011; 219(1): 253-8.

58. Cherfan P, Tompa A, Wikby A, Löfgren S, Jonasson L. Effects of simvastatin on human T cells in vivo. Atherosclerosis 2007; 193(1): 186-92.

59. Albert MA, Danielson E, Rifai N, Ridker PM, PRINCE Investigators. Effect of statin therapy on C-reactive protein levels: the pravastatin inflammation/CRP evaluation (PRINCE): a randomized trial and cohort study. JAMA 2001; 286(1): 64-70.

60. Ridker PM, Danielson E, Fonseca FA, et al. Rosuvastatin to prevent vascular events in men and women with elevated C-reactive protein. N Engl J Med 2008; 359(21): 2195-207.

61. Arora M, Chen L, Paglia M, et al. Simvastatin promotes Th2-type responses through the induction of the chitinase family member Ym1 in dendritic cells. Proc Natl Acad Sci U S A 2006; 103(20): 7777-82.

62. Fosso CK, Miller MJ, Solomon WR, Baker JR, Jr. Adverse effects of 3-hydroxy-3-methylglutaryl coenzyme A reductase inhibitors associated with elevated serum IgE and eosinophilia. J Allergy Clin Immunol 1995; 95(5 Pt 1): 1053-5

63. Mulder DJ, Van Haelst PL, Wobbes MH, et al. The effect of aggressive versus conventional lipid-lowering therapy on markers of inflammatory and oxidative stress. Cardiovasc Drugs Ther 2007; 21(2): 91-7.

64. Fehr T, Kahlert C, Fierz W, et al. Statin-induced immunomodulatory effects on human T cells in vivo. Atherosclerosis 2004; 175(1): 83-90

65. Van Haelst PL, Van Doormaal JJ, May JF, Gans RO, Crijns HJ, Tervaert JW. Secondary prevention with fluvastatin decreases levels of adhesion molecules, neopterin and C-reactive protein. Eur J Intern Med 2001; 12(6): 503-9.

66. Korpilähde T, Heliövaara M, Kaipiainen-Seppänen $\mathrm{O}$, Knekt P, Aho K. Regional differences in Finland in the prevalence of rheumatoid factor in the presence and absence of arthritis. Ann Rheum Dis 2003; 62(4): 353-5.

67. Tan EM, Feltkamp TE, Smolen JS, et al. Range of antinuclear antibodies in "healthy" individuals. Arthritis Rheum 1997; 40(9): 1601-11.

68. Hippisley-Cox J, Coupland C. Unintended effects of statins in men and women in England and Wales: population based cohort study using the QResearch database. BMJ 2010; 340: c2197.

69. Smeeth L, Douglas I, Hall AJ, Hubbard R, Evans S. Effect of statins on a wide range of health outcomes: a cohort study validated by comparison with randomized trials. Br J Clin Pharmacol 2009; 67(1): 99-109.

70. Mammen AL, Chung T, Christopher-Stine L, Rosen P, Rosen A, Doering KR, Casciola-Rosen LA. Autoantibodies against 3-hydroxy-3-methylglutaryl-coenzyme A reductase in patients with statin-associated autoimmune myopathy. Arthritis Rheum 2011; 63(3): 713-21. 
71. Mammen AL, Pak K, Williams EK, Brisson D, Coresh J, Selvin E, Gaudet D. Rarity of anti-3-hydroxy-3-methylglutaryl-coenzyme A reductase antibodies in statin users, including those with self-limited musculoskeletal side effects. Arthritis Care Res (Hoboken) 2012; 64(2): 269-72.

72. Chapman-Shimshoni D, Yuklea M, Radnay J, Shapiro H, Lishner M. Simvastatin induces apoptosis of B-CLL cells by activation of mitochondrial caspase 9. Exp Hematol 2003; 31(9): 779-83.

73. Mevorach D. Systemic lupus erythematosus and apoptosis: a question of balance. Clin Rev Allergy Immunol 2003; 25(1): 49-60.

74. Dieker JW, Van Der Vlag J, Berden JH. Deranged removal of apoptotic cells: its role in the genesis of lupus. Nephrol Dial Transplant 2004; 19(2): 282-5

75. Smeenk RJ. Antinuclear antibodies: cause of disease or caused by disease? Rheumatology (Oxford) 2000; 39(6): 581-4.

76. Fairweather D. Autoimmune disease: Mechanisms. In: Essential Immunology: Encyclopedia of Life Sciences. Delves PJ, Martin S, Burton DR, Roitt JM, eds. Wiley-Blackwell, Hoboken, 2007. pp. 1-6.

77. Haas D, Hoffmann GF. Mevalonate kinase deficiency and autoinflammatory disorders. N Engl J Med 2007; 356(26): 2671-3

78. Steffens S, Mach F. Drug insight: Immunomodulatory effects of statins--potential benefits for renal patients? Nature Clin Pract Nephrol 2006; 2(7): 378-87.

79. Schotte P, Denecker G, Van Den Broeke A, Vandenabeele P, Cornelis GR, Beyaert R. Targeting Rac1 by the Yersinia effector protein YopE inhibits caspase-1-mediated maturation and release of interleukin-1beta. J Biol Chem 2004; 279(24): 25134-42.

80. Coward WR, Marei A, Yang A, Vasa-Nicotera MM, Chow SC. Statin-induced proinflammatory response in mitogen-activated peripheral blood mononuclear cells through the activation of caspase-1 and IL-18 secretion in monocytes. J Immunol 2006; 176(9): 5284-92.

81. Lalor SJ, Dungan LS, Sutton CE, Basdeo SA, Fletcher JM, Mills KH. Caspase-1-processed cytokines IL-1beta and IL-18 promote IL-17 production by gammadelta and CD4 T cells that mediate autoimmunity. J Immunol 2011; 186(10): 5738-48.

82. Henneman L, Schneiders MS, Turkenburg M, Waterham HR. Compromized geranylgeranylation of RhoA and Rac1 in mevalonate kinase deficiency. J Inherit Metab Dis 2010; 33(5): 625-32.

83. The Lancet. Silicone breast implants: lessons from the USA. Lancet 2012; 379(9811): 93.

84. Kagami S, Owada $\mathrm{T}$, Kanari $\mathrm{H}$, et al. Protein geranylgeranylation regulates the balance between Th17 cells and Foxp3 regulatory T cells. Int Immunol 2009; 21(6): 679-89.

85. Mausner-Fainberg K, Luboshits G, Mor A, Maysel-Auslender S, Rubinstein A, Keren G, George J. The effect of HMG-CoA reductase inhibitors on naturally occurring CD4+CD25+ T cells. Atherosclerosis 2008; 197(2): 829-39.

86. Meng $\mathrm{X}$, Zhang $\mathrm{K}, \mathrm{Li} \mathrm{J}$, et al. Statins induce the accumulation of regulatory $\mathrm{T}$ cells in atherosclerotic plaque. Mol Med 2012; 18: 598-605.

87. Nilsson TK, Boman K, Jansson JH, Thogersen AM, Berggren M, Broberg A, Granlund A. Comparison of soluble thrombomodulin, von Willebrand factor, tPA/PAI-1 complex, and high-sensitivity CRP concentrations in serum, EDTA plasma, citrated plasma, and acidified citrated plasma (Stabilyte) stored at -70 degrees C for 8-11 years. Thromb Res 2005; 116(3): 249-54.

88. Aziz N, Nishanian P, Mitsuyasu R, Detels R, Fahey JL. Variables that affect assays for plasma cytokines and soluble activation markers. Clin Diagn Lab Immunol 1999; 6(1): 89-95.

89. Henderson CE, Ownby D, Klebanoff M, Levine RJ. Stability of immunoglobulin E (IgE) in stored obstetric sera. J Immunol Methods 1998; 213(1): 99-101.

90. Buurma H, Bouvy ML, De Smet PA, Floor-Schreudering A, Leufkens HG, Egberts AC. Prevalence and determinants of pharmacy shopping behaviour. J Clin Pharm Ther 2008; 33(1): 17-23. 


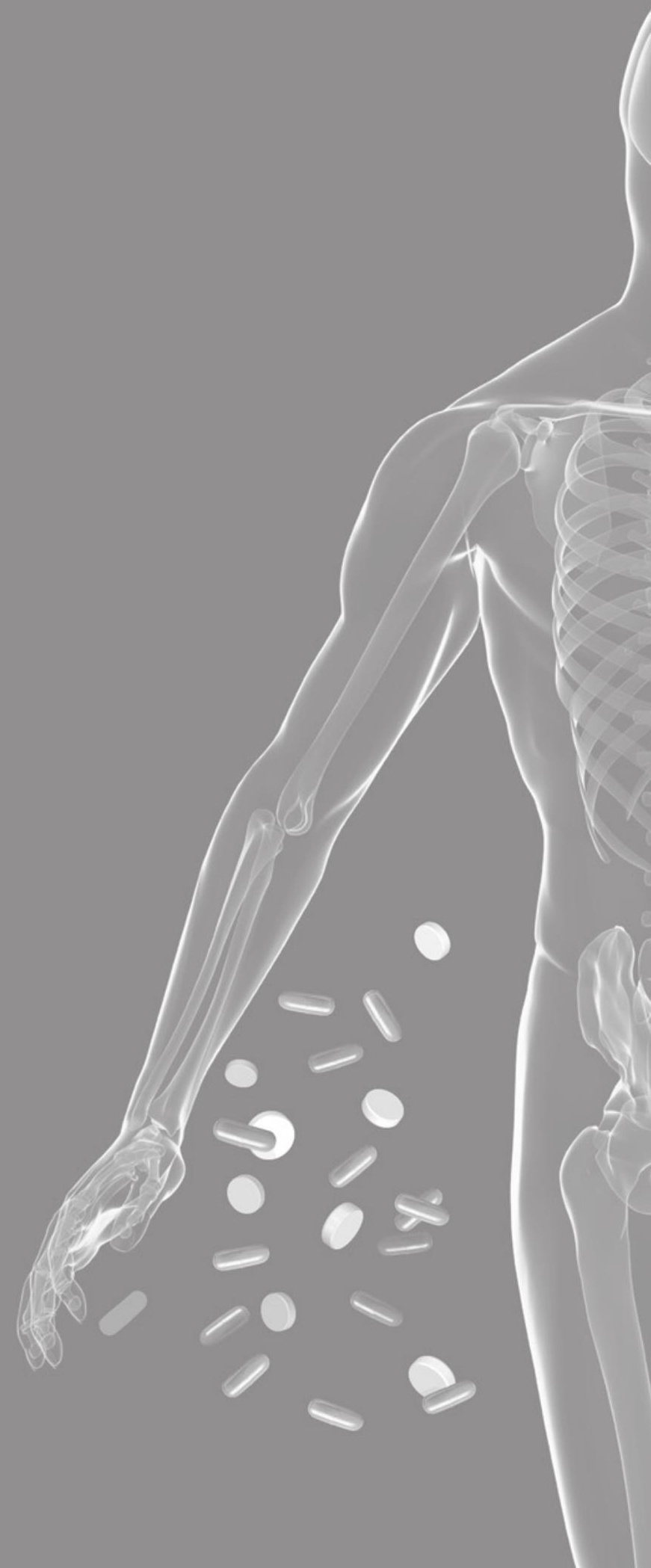




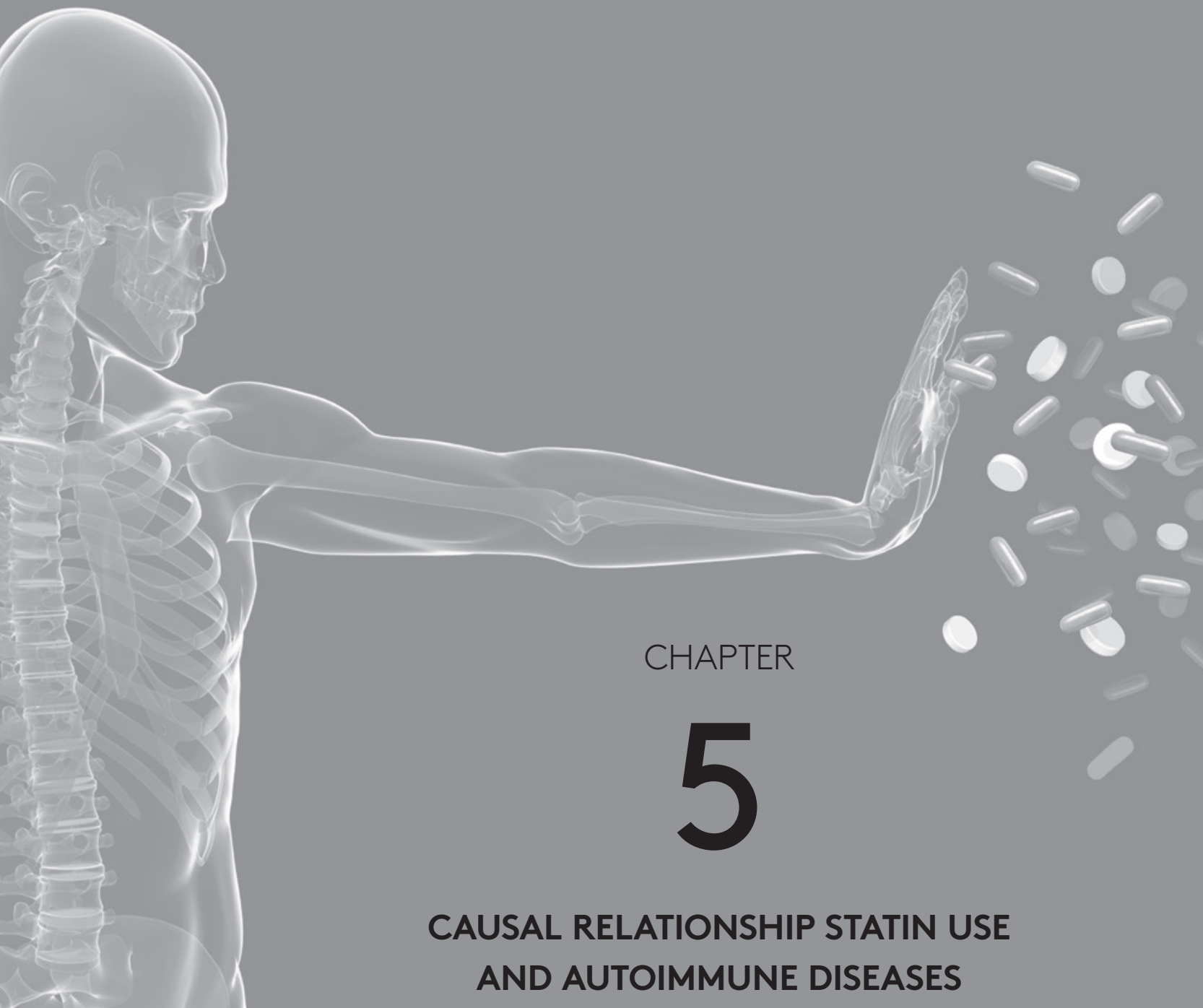




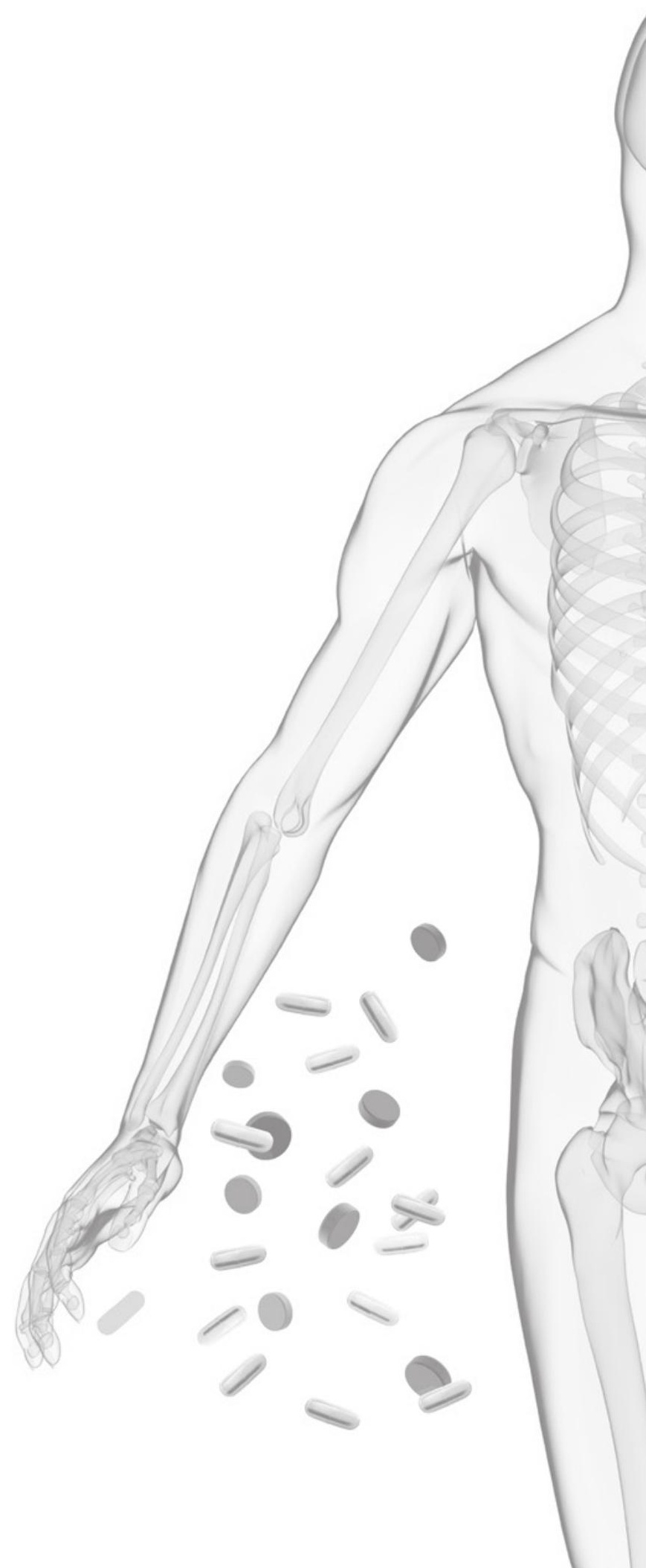




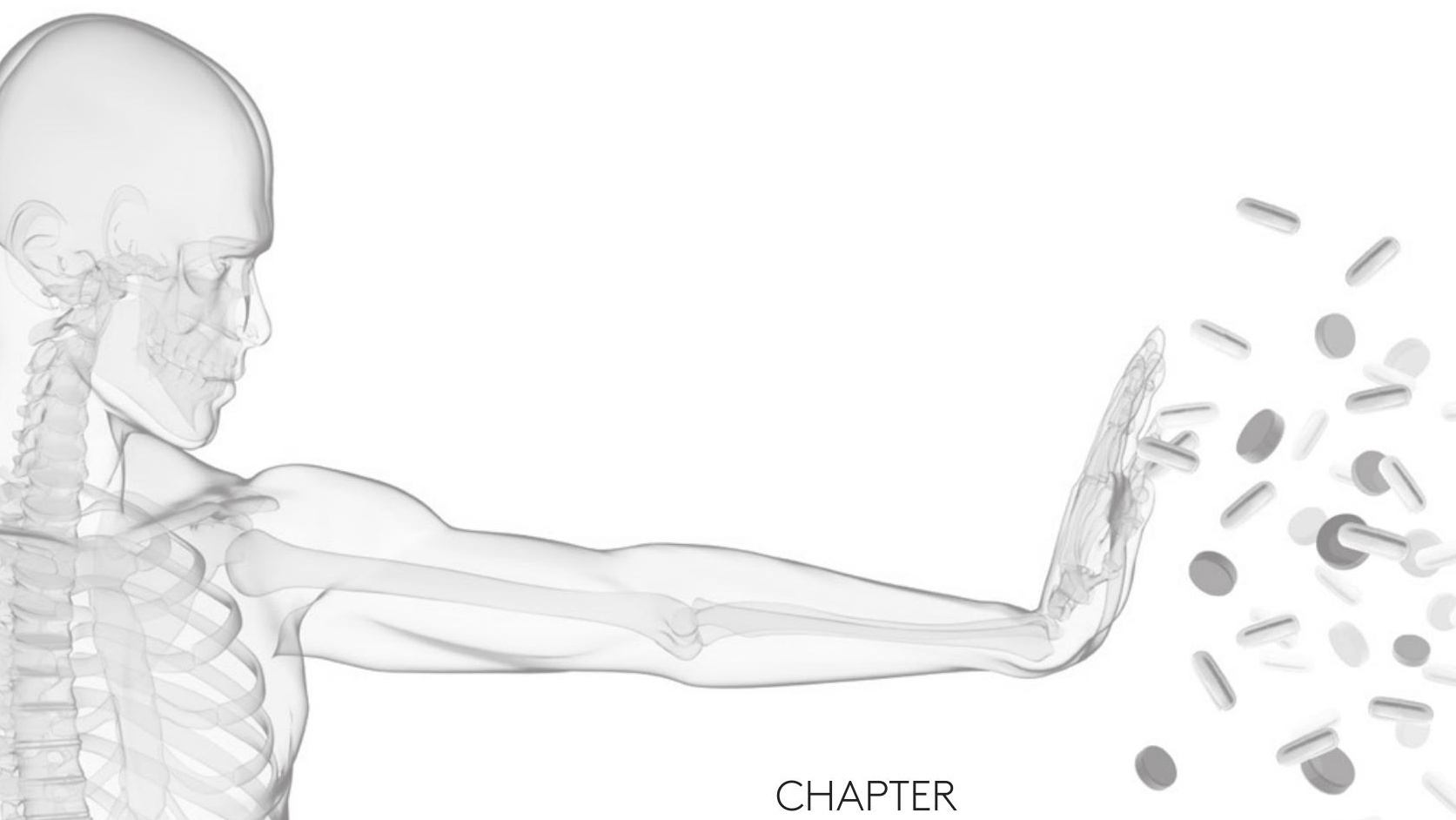

CHAPTER

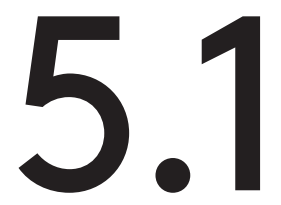

\title{
STATINS ACCELERATE THE ONSET OF COLLAGEN TYPE II-INDUCED ARTHRITIS IN MICE
}

\author{
R. J. Vandebriel, H. J. I. De Jong, E. R. Gremmer, O. H. Klungel, JW. Cohen \\ Tervaert, \\ W. Slob, J. W. Van Der Laan, H. Van Loveren.
}

Arthritis Res Therapy 2012; 14(2): R90 


\section{ABSTRACT}

\section{Introduction}

Statins (3-hydroxy-3-methylglutaryl coenzyme A reductase inhibitors) are effective in reducing the risk of cardiovascular morbidity and mortality in patients with hyperlipidaemia, hypertension, or diabetes mellitus type II. Next to their cholesterol-lowering activity, statins have immunomodulatory properties. Based on these properties, we hypothesised that statin use may eventually lead to dysregulation of immune responses, possibly resulting in autoimmunity. We have recently shown in an observational study that statin use was associated with an increased risk of developing rheumatoid arthritis. Our objective was to investigate whether a causal relationship could be established for this finding.

\section{Methods}

The mouse collagen type II (CII)-induced arthritis (CIA) model was used, with immunisation, challenge and euthanasia at days 0,21 and 42, respectively. Statins were given orally before (day -28 until day 21) or after (day 21 until day 42) CIA induction. Atorvastatin (0.2 mg/day) or pravastatin ( $0.8 \mathrm{mg} /$ day) was administered. Arthritis was recorded three times a week. Serum anti-Cll autoantibodies and cytokines in supernatants from Concanavalin-A-stimulated lymph node cells and Cll-stimulated spleen cells were measured.

\section{Results}

Statin administration accelerated arthritis onset and resulted in 100\% arthritic animals, whereas only seven out of twelve non-statin animals (controls) developed arthritis. Atorvastatin administration after CIA induction resulted in earlier onset than atorvastatin administration before induction, or than pravastatin administration before or after induction. The arthritic score of animals given pravastatin before CIA induction was similar to that of the non-statin controls, whereas the other groups that received statins showed higher arthritic scores. Atorvastatin administration, especially before CIA induction, increased anti-CII autoantibody production. Interleukin (IL)-2 and IL-17 production by lymph node and spleen cells was higher in CIA animals than in Phosphate buffered saline (PBS) controls, but was not affected by statin administration. While interferon- $\gamma$ (IFN- $\gamma$ ) production was not affected by CIA induction, atorvastatin administration before CIA induction increased the production of this cytokine.

\section{Conclusion}

These data support previous results from our observational studies, indicating a role for statins in the induction of autoimmunity. 


\section{INTRODUCTION}

Statins (3-hydroxy-3-methylglutaryl coenzyme A reductase inhibitors) have been shown to be effective in reducing the risk of cardiovascular morbidity and mortality in patients with hyperlipidaemia, hypertension, or diabetes mellitus type II ${ }^{1-4}$. In addition to their cholesterol-lowering activity, several studies have shown that statins have anti-inflammatory and immunomodulatory properties ${ }^{5-8}$. We hypothesised that because of these immunomodulatory properties statin use may eventually lead to dysregulation of immune responses, possibly resulting in autoimmunity. In line with this hypothesis, we have recently shown in an observational study that statin use was associated with an increased risk of developing rheumatoid arthritis (RA) ${ }^{9}$. Moreover, based on individual case reports we found a positive association between statin use and the occurrence of lupus-like syndrome ${ }^{10}$. This latter association was recently confirmed by another research group ${ }^{11}$. To investigate whether a causal relationship can be established for these observations, we evaluated the effects of statin administration on arthritis in the collagen type II (CII)-induced arthritis (CIA) mouse model. In this model for RA, mice are immunised with Cll mixed with Freund's complete adjuvant, and are challenged three weeks later with $\mathrm{Cll}$ alone. Arthritis is scored from the time of challenge onwards ${ }^{12}$. Several studies have shown beneficial effects of statin administration on joint inflammation in the mouse CIA model ${ }^{13-16}$. However, these studies did not specifically address the effects of statin administration before arthritis induction, an issue that follows from our observational study 9 . Nonetheless, to relate our results to the animal studies indicated above ${ }^{13-16}$, we also evaluated effects of statin administration after arthritis induction. Here we show that statin administration accelerated arthritis onset and resulted in 100\% arthritic animals, whereas only seven out of twelve non-statin animals (controls) developed arthritis. Atorvastatin administration after CIA induction resulted in earlier onset than atorvastatin administration before induction, or than pravastatin administration before or after induction. The arthritic score of animals given pravastatin before CIA induction was similar to that of the non-statin controls, whereas the other groups that received statins showed higher arthritic scores. Atorvastatin administration, especially before CIA induction, increased anti-CII autoantibody production. Interleukin (IL)-2 and IL-17 production by lymph node $(L N)$ and spleen cells was not affected by statin administration. Atorvastatin administration before CIA induction increased interferon- $\gamma$ (IFN- $\gamma$ ) production. 


\section{MATERIALS AND METHODS}

\section{Induction and assessment of collagen-induced arthritis}

Male DBA/1OlaHsd mice were obtained from Harlan (Horst, the Netherlands). At the age of 10 to 12 weeks the animals were injected intradermally at the base of the tail with $100 \mu \mathrm{l}$ of an emulsion of bovine Cll (Chondrex, Redmond, WA, USA) and Freund's complete adjuvant (Chondrex) on day 0 (final concentrations of $\mathrm{Cll}$ and Freund's complete adjuvant, $1 \mathrm{mg} / \mathrm{ml}$ ). Mice were challenged by intra-peritoneal injection of $100 \mu \mathrm{l} \mathrm{Cll}$ (concentration $1 \mathrm{mg} / \mathrm{ml}$ ) on day 21. Body weight and arthritis severity were assessed three times per week in a blinded manner, using a semi-quantitative scoring system (with scores ranging from 0 to 4 for each paw) until mice were euthanized on day 4212 . Arthritis scoring was performed by two individuals, alternating between days; there were no visible systematic differences in the way they scored. Mice were anesthetised with ketamine, rompun and atropine, and blood was collected from the orbital plexus. Inguinal LNs of the hind paws and the spleens were excised. The animal experiments were approved prior to their commencement by an independent ethical committee, in accordance with national legislation.

\section{Statin administration}

Atorvastatin (calcium salt) was a kind gift from Pfizer (Groton, CT, USA). Pravastatin (sodium salt) was obtained from Teva (Debrecen, Hungary). Atorvastatin $(1 \mathrm{mg} / \mathrm{ml})$ and pravastatin $(4 \mathrm{mg} / \mathrm{ml})$ were administered daily by oral gavages $(0.2 \mathrm{ml})$. The atorvastatin dose approximates $10 \mathrm{mg} / \mathrm{kg}$ body weight 17 . The cholesterol-lowering and low-density lipoprotein-lowering efficacy of pravastatin is four times lower than that of atorvastatin 18 , and pravastatin was thus given at a four times higher concentration than atorvastatin. Atorvastatin and pravastatin were given either before challenge (day -28 until day 20) or after challenge (day 22 until day 42; see Figure 1). Phosphate buffered saline (PBS) $(0.2 \mathrm{ml})$ was given as a control on days when statins were not administered. One control group received only PBS, and a second control group (negative control) received only PBS and arthritis was not induced. These groups received $0.2 \mathrm{ml}$ PBS by oral gavages daily throughout the experiment. There were 12 mice per group.

\section{Measurement of autoantibodies}

Anti-bovine CII immunoglobulin G (IgG) antibodies were measured using an ELISA (mouse anti-bovine Cll IgG assay kit; Chondrex) according to the manufacturer's instructions. Briefly, after incubation with blocking buffer, a serial dilution of the standard as well as a 20000 -fold dilution of serum samples was incubated. After incubation with peroxidase-conjugated goat anti-mouse IgG, O-phenylenediamine dihydrochloride was added, the reaction was stopped, and the optical density was 
measured at $490 \mathrm{~nm}$. The plates were washed before and between all incubation steps.

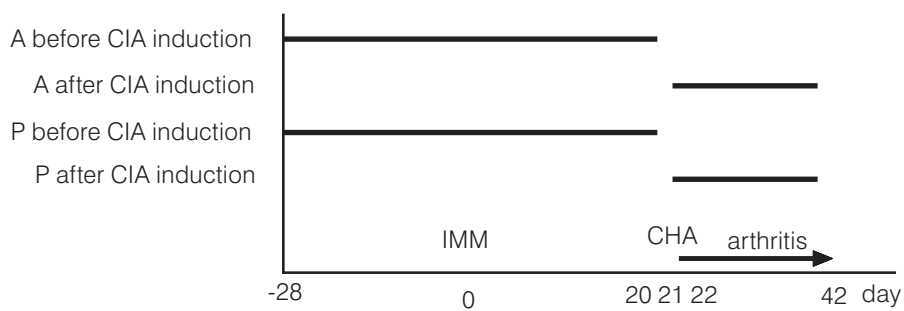

Figure 1 Scheme of CIA induction and statin administration.

Mice were immunised at day 0 (IMM) and challenged at day $21(\mathrm{CHA})$, after which arthritis was scored 3 times a week until day 42. Atorvastatin (A) or pravastatin (P) were given daily, either before (day -28 until day 20) or after CIA induction (day 22 until day 42). Mice were euthanized at day 42.

\section{Cell culture}

The culture medium used was RPMI-1640 (Gibco, Grand Island, NY, USA) supplemented with $10 \% \mathrm{FCS}, 100 \mu \mathrm{g} / \mathrm{ml}$ streptomycin and $100 \mathrm{IU} / \mathrm{ml}$ penicillin. Cell suspensions were made by pressing the LNs and spleens through a cell strainer (Falcon, Franklin Lakes, NJ, USA). Cells were counted using a Coulter Counter (Coulter Electronics, Luton, UK). LN cell suspensions were cultured at 106 cells/ml culture medium with $5 \mu \mathrm{g} / \mathrm{ml}$ Concanavalin A (MP Biomedicals, Irvine, CA, USA) in 96-well tissue culture plates (Nunc, Roskilde, Denmark) for 24 hours. Spleen cell suspensions were cultured at 106 cells $/ \mathrm{ml}$ culture medium with $50 \mu \mathrm{g} / \mathrm{ml}$ Cll or $5 \mu \mathrm{g} /$ $\mathrm{ml}$ Concanavalin A in 96-well tissue culture plates (Nunc) for 72 hours. Culture conditions were $37^{\circ} \mathrm{C}$ in a humidified atmosphere containing $5 \%$ carbon dioxide.

\section{Cytokine measurements}

A 10-plex panel containing beads for mouse IL-13, IL-2, IL-4, IL-6, IL-10, IL-12p70, IL-17, granulocyte-macrophage colony-stimulating factor, IFN- $\gamma$ and TNF-a (Bio-Rad, Hercules, CA, USA) was used. After incubation and washing steps ${ }^{19}$, the beads were measured on a Bio-Plex (Bio-Rad).

\section{Statistical analysis of arthritis induction}

We analysed the fraction of animals with arthritis as a function of time, by fitting timeresponse models to our data. We used PROAST, a general program for dose-response modelling 20,21. These models apply directly by regarding time rather than dose as the independent variable - that is, the time of onset of arthritis defined as the median 
time when $50 \%$ of the animals develop arthritis (ET50). The fitted model was the log-logistic model:

$y=a+(1-a) /\{1+\exp [c \ln (b / x)]\}$

where $y$ is the fraction of animals showing arthritis and $x$ is the time $(a, b$ and $c$ are constants). First, the model was fitted to the data from all (five) experimental groups combined - that is, the same ET50 value was estimated for all treatment groups. The same model was then fitted but now by estimating a treatment-specific ET50 value. When the associated fit of the model (as reflected by the value of the log-likelihood) is significantly improved (likelihood ratio test), this indicates a significant treatment effect on the ET50 value.

The analysis of the fraction of animals with arthritis over time as described above is statistically invalid because the observations over time relate to the same animals (that is, the assumption of independent observations does not hold). As a result, $p$ values and confidence intervals of the means will be too optimistic. We therefore performed an additional analysis as follows. The arthritis severity scores were analysed as a function of time using a so-called latent-variable model. In this model the latent variable reflects the arthritis severity as a continuously increasing value with time, while the observed scores are imagined to arise from consecutive cut-off values on that underlying continuous severity scale. These cut-off values are estimated while fitting the time-response model to the scores 22.

The latent-variable model:

$y=a \exp (b \times d)$

where $y$ is the latent variable and $x$ is time ( $a, b$ and $d$ are constants), was fitted to the observed scores for all individuals combined, by including the factor individual animal as a covariate. Individuals were found to significantly differ in the time-response curve regarding parameter $b$, but parameters a and $d$ were not found to differ significantly. Hence, we fitted the model with only parameter b dependent on the individual animal. The resulting estimates for $b$ were then analysed in a one-way analysis of variance to compare the treatments. In this analysis the estimates for $b$ are statistically independent. Because the results of statin treatments on arthritis induction using the latent variable model were similar to the results using the log-likelihood model, we have chosen to show the results of the latter model.

\section{General statistical analysis}

The effect of arthritis induction on body weight, autoantibody production and cytokine production was assessed using an independent samples t test (SPSS Inc., Chicago, 
Illinois, USA). The effect of statin treatments on body weight, autoantibody production and cytokine production was assessed using one-way analysis of variance, followed by the Bonferroni post hoc test (SPSS Inc.).

\section{RESULTS}

\section{Body weight}

Induction of CIA resulted in a lower body weight compared with negative control animals (Figure 2). This difference was statistically significant $(p<0.05)$ from day 33 after immunisation onwards. Atorvastatin administration before $\mathrm{CIA}$ induction resulted in a lower body weight compared with CIA control animals (Figure 2). This difference was statistically significant $(p<0.05)$ from day 37 after immunisation onwards. Atorvastatin administration after $\mathrm{CIA}$ induction, or pravastatin administration before or after CIA induction, did not significantly alter body weight compared with CIA control animals.

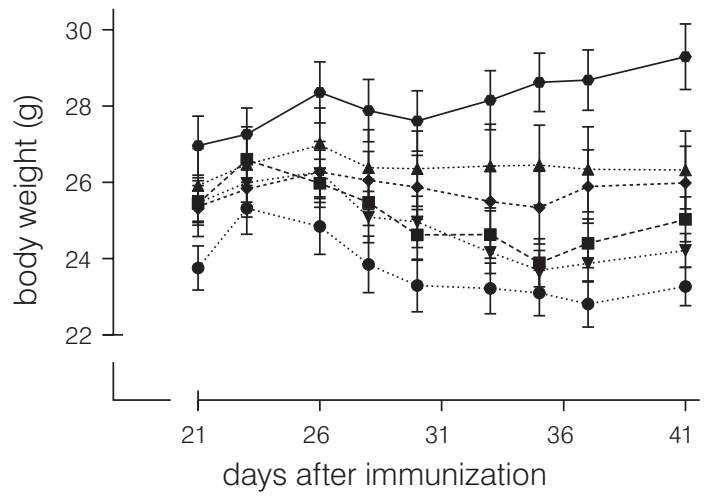

Figure 2 Effect of CIA induction and statin treatment on body weight.

Mice were immunised at day 0 , challenged at day 21, and weighed 3 times a week until euthanasia at day 42. Statins were given daily. Black circles connected by dotted line: atorvastatin before CIA induction. Black squares connected by dotted line: atorvastatin after CIA induction. Black triangles connected by dotted line: pravastatin before CIA induction. Black upside-down triangles connected by dotted line: pravastatin after $\mathrm{CIA}$ induction. Black diamonds connected by dotted line: CIA control. Black circles connected by continuous line: negative control. $N=12$ per group. The effect of arthritis induction was assessed using an independent samples t-test; the effect of statin treatments was assessed using one-way ANOVA, followed by the Bonferroni post-hoc test. 


\section{Arthritis}

Statin administration (both atorvastatin and pravastatin, both before and after CIA induction) resulted in 100\% arthritic animals at the time of euthanasia, whereas only seven out of twelve non-statin animals (controls) had developed arthritis at that time point (Figure 3A). Statin administration resulted in earlier arthritis onset, expressed as the day after immunisation in which $50 \%$ of the animals show arthritis (Figure 4).

A

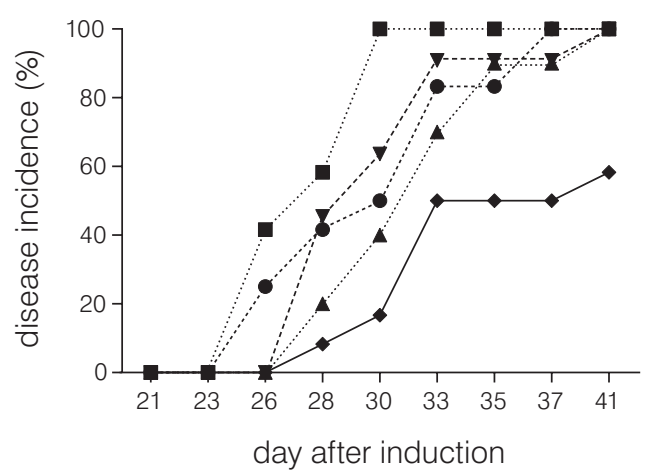

B

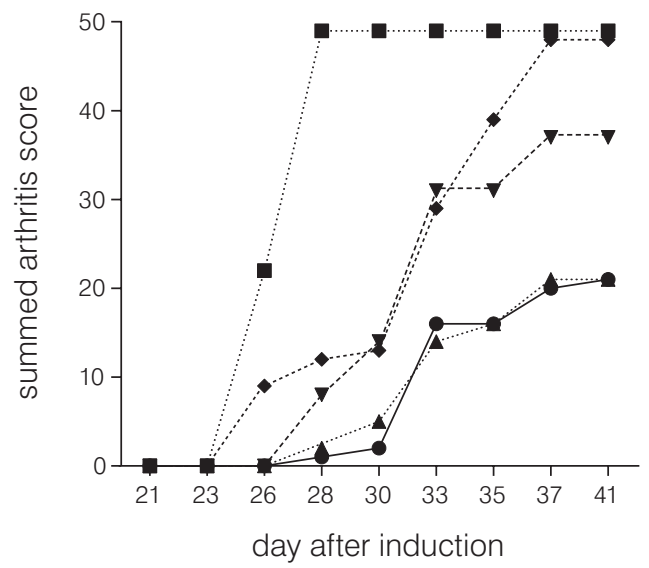

Figure 3 Effect of statin treatment on arthritis incidence and score.

(A) Number of arthritic animals per group vs. time after immunisation.

(B) Summed arthritis score per group of animals vs. time after immunisation. Mice were immunised at day 0 and challenged at day 21, after which arthritis was scored 3 times a week until day 42 . Statins were given daily. Black circles connected by dotted line: atorvastatin before CIA induction, Black squares connected by dotted line: atorvastatin after CIA induction, Black triangles connected by dotted line: pravastatin before CIA induction, Black upside-down triangles connected by dotted line: pravastatin after CIA induction. Black diamonds connected by continuous line: CIA control. $\mathrm{N}=12$ per group. 


\section{A}

\section{Response}

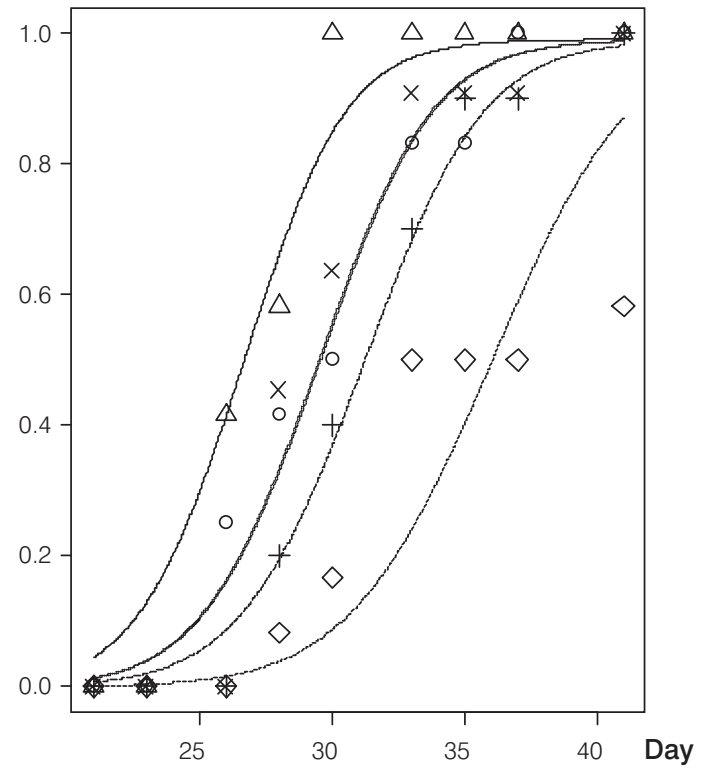

B

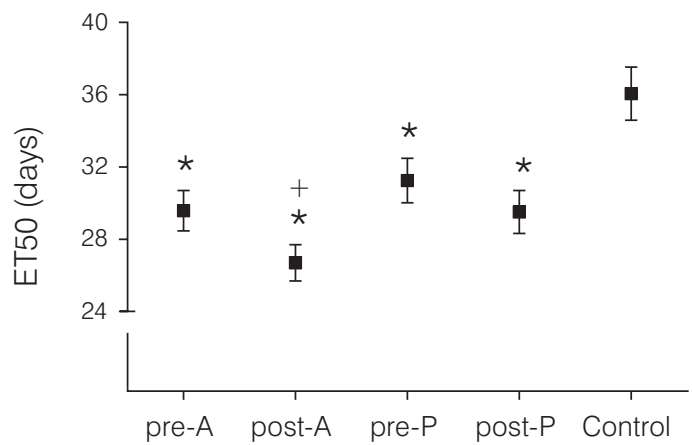

Figure 4 Statin administration accelerates arthritis onset (fraction of arthritic animals vs. time).

Mice were immunised at day 0 and challenged at day 21, after which arthritis was scored 3 times a week until day 42. (A) Mice were given atorvastatin before challenge (days -28 until day 20; pre-A; cross sign, grey line) or after challenge (days 22 until 42; post-A; open triangle, dark grey line), or they were given pravastatin before challenge (pre-P; plus sign, dashed line) or after challenge (post-P; open circle, black line). Control mice (open diamond, dotted line) did not receive statins. The grey and black lines are overlapping.

(B) The ET50 values (mean and 90\% confidence intervals) calculated from Fig.4A are depicted. $\left(^{*}\right)$ significantly different $(p<0.05)$ from control mice; $(+)$ significantly different $(p<0.05)$ from pre-A, pre-P, and post- $P$ treated mice. $N=12$ per group. The ET50 values (mean and $90 \%$ confidence intervals) were calculated using the PROAST program. One-way ANOVA followed by the Bonferroni post-hoc test. 
Atorvastatin administration after $\mathrm{CIA}$ induction resulted in earlier onset than atorvastatin administration before CIA induction, or than pravastatin administration before or after CIA induction (Figure 4).

The arthritic score summed per group of animals showed a similar score for the animals that received pravastatin before CIA induction and non-statin controls, whereas the other groups of statin-administered animals showed higher scores (Figure 3B). Only seven out of twelve non-statin controls became arthritic, so the mean arthritic score for animals that received pravastatin before CIA induction was lower than for the non-statin controls (data not shown).

\section{Autoantibody production}

Induction of CIA resulted in the presence of anti-Cll antibodies (log IgG level = 5.17; $\mathrm{p}<0.001$ compared with the negative controls). Atorvastatin administration resulted in higher anti-Cll antibody production compared with pravastatin administration and the CIA control ( $p=0.005$ and $p=0.013$, respectively). In addition, atorvastatin administration before CIA induction increased the anti-Cll antibody production compared with the CIA control $(p<0.05$; Figure 5$)$.

\section{Cytokine production}

Induction of CIA resulted in increased IL-2 production by Concanavalin-A-stimulated LN cells and increased IL-2 and IL-17 production by Cll-stimulated spleen cells $(p<0.05$; Figure 6$)$. For none of the other cytokines tested was a significant effect due to $\mathrm{CIA}$ induction found.

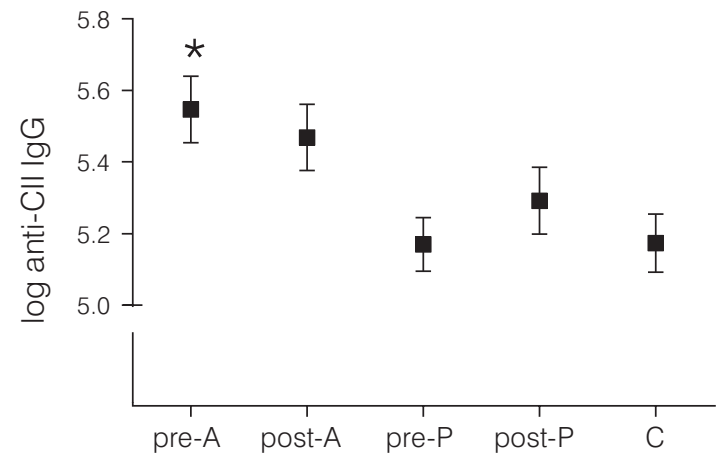

Figure 5 Anti-collagen type II IgG levels.

Mice were immunised at day 0 and challenged at day 21. Atorvastatin $(A)$ or pravastatin $(P)$ were given daily, either before (day -28 until day 20) or after (day 22 until day 42) CIA induction. Mice were euthanized at day 42. Control mice $(\mathrm{C})$ did not receive statins. Anti-CII IgG was measured in serum by ELISA. $N=12 .\left(^{*}\right)$ Significantly different $(p<0.05)$ from $C . N=12$ per group. One-way ANOVA followed by the Bonferroni post-hoc test. 
Atorvastatin administration after CIA induction increased IFN- $\gamma$ production by Cll-stimulated spleen cells ( $p<0.05$; Figure 6$)$. Combining the results of both statins, administration after but not before CIA induction resulted in increased production $(p<0.01)$. Combining the results of both time points, both atorvastatin and pravastatin increased IFN- $\gamma$ production $(\mathrm{P}<0.05)$. For none of the other cytokines tested was a significant effect due to statin administration found.

A

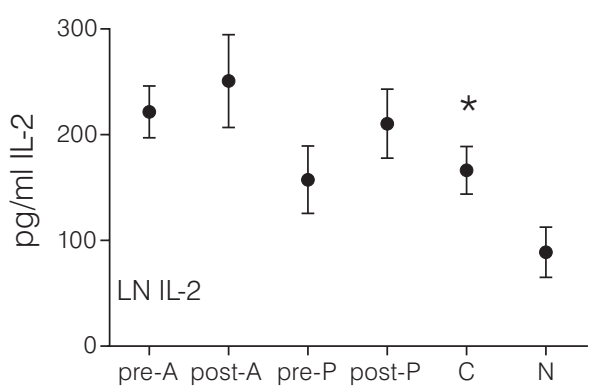

B

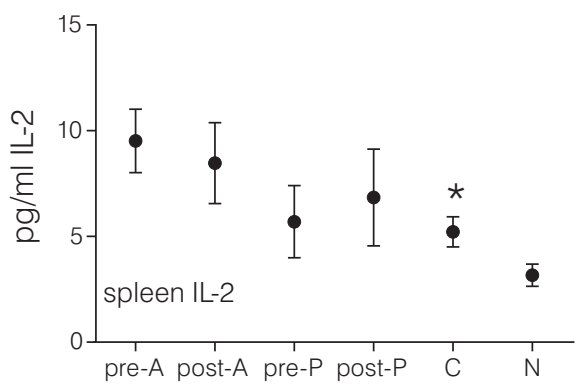

D

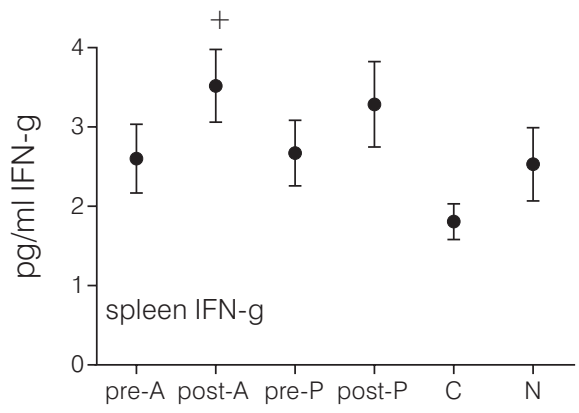

Figure 6 Cytokine productions by LN and spleen cells.

(A) IL-2 production by Con A-stimulated inguinal LN cells. (B) IL-2 production by collagen type II-stimulated spleen cells. (C) IL-17 production by collagen type II-stimulated spleen cells. (D) IFN-production by collagen type II-stimulated spleen cells. Mice were immunised at day 0 and challenged at day 21. Atorvastatin (A) or pravastatin (P) were given daily, either before (day -28 until day 20) or after (day 22 until day 42) CIA induction. Mice were euthanized at day 42. Control mice (C) did not receive statins. In negative mice $(\mathrm{N})$ arthritis was not induced and these mice did not receive statins. Inguinal LN were excised and LN cells were cultured in the presence of Con A for 24 hours. Spleens were excised and spleen cells were cultured in the presence of Cll for 72 hours. Cytokine content was measured in the supernatants. $N=12$. $\left(^{*}\right)$ Significantly different $(p<0.05)$ from negative mice $(N) .(+)$ Significantly different $(p<0.05)$ from control mice $(C) . N=12$ per group. The effect of arthritis induction was assessed using an independent samples t test; the effect of statin treatments was assessed using one-way ANOVA, followed by the Bonferroni post-hoc test. 


\section{DISCUSSION}

Here we have shown that statin administration results in $100 \%$ arthritic animals, whereas only seven out of twelve non-statin animals (controls) developed arthritis. Moreover, statin administration accelerated arthritis onset. This accelerated onset was seen when statin administration was started either before or after CIA induction. Accelerated onset resulting from statin administration before CIA induction is in line with our hypothesis of statin-induced immune deviation resulting in autoimmunity 9,10. In addition, we observed that atorvastatin administration after CIA induction resulted in earlier arthritis induction than administration with the same statin before CIA induction. Overall, atorvastatin had a somewhat stronger effect on accelerating onset than pravastatin. Atorvastatin administration before or after CIA induction, or pravastatin administration after CIA induction, resulted in a similar mean arthritic score compared with the non-statin control, while pravastatin administration before CIA induction resulted in a lower mean arthritic score. In this latter pravastatin group the acceleration of arthritis onset was five days, while that in the other cases was seven or nine days. This lower mean arthritic score may suggest a beneficial effect of pravastatin. This dosing regime, however, also results in accelerated arthritis onset and an increased number of arthritic animals (mice).

Other studies using the mouse CIA model have shown beneficial effects of statin administration on joint inflammation 13-16. To establish whether statin type, dose, route of administration, or timing of administration relative to $\mathrm{CIA}$ induction influenced the clinical outcome, the results of the abovementioned studies, as well as our data, are compared in Table 1. Suppression of arthritis development was seen after oral administration of $10 \mathrm{mg} / \mathrm{kg}$ atorvastatin (the same exposure route and dose as we used in our study) during (almost) the whole period from immunisation to euthanasia 13. This finding contrasts our results that show accelerated onset when $10 \mathrm{mg} / \mathrm{kg}$ atorvastatin was given orally either before or after $\mathrm{Cll}$ challenge. This issue needs to be resolved in future studies. Suppression of arthritis development was also found after intraperitoneal administration of $100 \mathrm{mg} / \mathrm{kg}$ pravastatin ${ }^{14}$ or $40 \mathrm{mg} / \mathrm{kg}$ simvastatin 15,16. Using other statins, lower doses, or other exposure routes failed to show an effect on arthritis. An accelerated onset of arthritis was only found in our study.

Atorvastatin administration before CIA induction resulted in increased anti-CII antibody production, whereas atorvastatin administration after CIA induction resulted in a non-significant increase in production of this antibody. Pravastatin administration before and after CIA induction, however, did not affect anti-CII IgG antibody production. Atorvastatin administration before CIA induction resulted in accelerated arthritis onset and increased autoantibody production. This observation may suggest that increased autoantibody production plays a role in accelerated arthritis onset. Our observational study showed that statin use is associated with an increased risk of developing RA 9. 
Table 1 Comparison of statin effects on murine collagen-induced arthritis

\begin{tabular}{|c|c|c|c|c|c|}
\hline Study & Statin & $\begin{array}{l}\text { Dose } \\
(\mathrm{mg} / \mathrm{kg})\end{array}$ & $\begin{array}{l}\text { Exposure } \\
\text { route }\end{array}$ & $\begin{array}{l}\text { Timing } \\
\text { (days) }\end{array}$ & $\begin{array}{l}\text { Effect on } \\
\text { arthritis }\end{array}$ \\
\hline This study & $A^{*}$ & 10 & Oral & -28 till 20 & $\Delta$ ET50 -7 days \\
\hline This study & A & 10 & Oral & 22 till 42 & $\Delta$ ET50 -9 days \\
\hline This study & $\mathrm{P}^{\dagger}$ & 40 & Oral & -28 till 20 & $\Delta$ ET50 - 5 days \\
\hline This study & $P$ & 40 & Oral & 22 till 42 & $\Delta \mathrm{ET} 50-7$ days \\
\hline Ho and colleagues. ${ }^{13}$ & A & 10 & Oral & 2 till 49 & suppression \\
\hline Yamagata and colleagues. ${ }^{14}$ & $\mathrm{P}$ & 100 & intraperitoneal & 0 till 35 & suppression \\
\hline Leung and colleagues. ${ }^{15}$ & $S^{\ddagger}$ & 40 & intraperitoneal & 21 till 40 & suppression \\
\hline Leung and colleagues. ${ }^{15}$ & S & 20 & intraperitoneal & 21 till 40 & no effect \\
\hline Leung and colleagues. ${ }^{15}$ & S & 10 & intraperitoneal & 21 till 40 & no effect \\
\hline Palmer and colleagues. ${ }^{16}$ & A & 100 & Oral & 0 till 48 & no effect \\
\hline Palmer and colleagues. ${ }^{16}$ & A & 1 & Oral & 0 till 48 & no effect \\
\hline Palmer and colleagues. ${ }^{16}$ & $\mathrm{R}$ & 2 & subcutaneous & 0 till 48 & no effect \\
\hline Palmer and colleagues. ${ }^{16}$ & $\mathrm{R}^{\S}$ & 0.2 & subcutaneous & 0 till 48 & no effect \\
\hline Palmer and colleagues. ${ }^{16}$ & $\mathrm{R}$ & 40 & intraperitoneal & 21 till 35 & no effect \\
\hline Palmer and colleagues. ${ }^{16}$ & S & 40 & intraperitoneal & 21 till 35 & suppression \\
\hline Palmer and colleagues. ${ }^{16}$ & $S$ & 40 & Oral & 21 till 35 & no effect \\
\hline
\end{tabular}

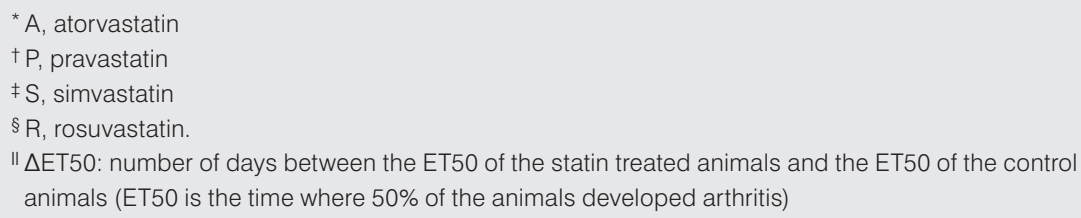

In humans, IgM rheumatoid factor and anti-cyclic citrullinated peptide antibodies have been found years before RA becomes clinically apparent 23 . For RA patients that used statins before developing clinically manifest disease, therefore, statin use may have coincided with autoantibody development. Statins may be speculated to increase autoantibody production, thereby facilitating RA development.

CIA induction resulted in increased IL-2 production by Concanavalin-A-stimulated LN cells, probably reflecting the stronger activation or higher frequency of $T$ cells in the LN draining the inflamed joints. Production of this cytokine was not significantly affected by statin administration. Similarly, CIA induction resulted in increased IL-2 production by Cll-stimulated spleen cells, and production of IL-2 was not significantly affected by statin administration. 
Furthermore, CIA induction resulted in increased IL-17 production by Cll-stimulated spleen cells. Since IL-23-deficient mice that lack IL-17-producing CD4+ cells (T helper (Th) 17 cells) are resistant to CIA induction 24, Th17 cells are assumed to play an essential role in CIA. The activation or frequency of Th17 cells in the spleen is probably increased in arthritic mice, in line with previous studies 24-27. Importantly, IL-17 production was not significantly affected by statin administration.

In addition, CIA induction resulted in decreased IFN- $\gamma$ production by $L N$ cells (0.75-fold) and Cll-stimulated spleen cells (0.71-fold), albeit not statistically significantly (data not shown). Since IFN- $\gamma$ acts as a disease-limiting factor in CIA 28 , reduced levels of this cytokine may possibly be characteristic for CIA. Since atorvastatin was shown to inhibit IFN- $\gamma$ production ${ }^{29}$, and absence of IFN- $\gamma$ signalling resulted in accelerated arthritis onset 30,31 , reduced production of this cytokine may be a possible mechanism underlying accelerated arthritis onset due to atorvastatin treatment. We did not, however, observe altered IFN- $\gamma$ production due to statin administration. Taking together the IL-17 and IFN- $\gamma$ data, the increased IL-17 and decreased IFN- $\gamma$ production upon CIA induction fits the selective induction of Th17 and not Th1 cells in CIA 24 .

Another possible mechanism by which statins may affect arthritis is through their induction of caspase-1, IL-1 $\beta$ and IL-18 in monocytes, resulting in increased IFN- $\gamma$ production by $T$ cells ${ }^{32}$. Interestingly, this increased IFN- $\gamma$ production occurred only at low statin concentrations. Caspase- 1 activation results in cleavage of pro-IL-1 $\beta$ and pro-IL-18 to their mature (active) forms. Caspase-1, IL-1 $\beta$ and IL-18 are all implicated in $\mathrm{CIA}$. The pharmacologic caspase-1 inhibitor $\mathrm{VX}-765$ reduced paw inflammation when given prophylactically and therapeutically in mice ${ }^{33}$. IL-1 3 administration accelerated arthritis 34 , while anti-IL-1 $\beta$ treatment markedly suppressed established arthritis 35 . IL-18 administration resulted in higher arthritis incidence and severity 36,37 , while IL-18-deficient mice showed reduced arthritis incidence and severity 38. This observation may suggest that statins aggravate arthritis by activating caspase-1, thereby inducing IL-1 $\beta$ and IL-18. Increased IFN- $\gamma$ production may, on the contrary, reduce arthritis. Taking the two possible mechanisms together, caspase-1 activation by statins may aggravate arthritis by inducing IL-1 13 and IL-18. The resulting IFN- $\gamma$ induction that in itself reduces arthritis may be counteracted by the Th2 skewing effect of statins. Clearly, disease outcome results from a complicated interplay between various effects of statins. In our hands, statin exposure resulted in accelerated arthritis onset.

In summary, the anti-CII antibody, IL-2, IL-17 and IFN- $\gamma$ data do not provide a possible mechanism for the accelerated arthritis observed in this study. It should be noted, however, that establishing such a mechanism was not the aim of our study. We aimed to investigate whether a causal relationship could be established for the association between statin use and an increased risk of developing RA. 
The CIA model was chosen because our observational study has shown that statin use was associated with an increased risk of developing $\mathrm{RA}^{9}$; because it is a model for RA, recapitulating many aspects of the disease $39-42$; because an induced model is preferred to a spontaneous model, since that is the only model where the effects of statin use prior to expression of arthritis can be tested; and because the mouse CIA model is both B-cell and T-cell mediated and is therefore more similar to human RA than models such as the rat adjuvant arthritis model, which is (only) T-cell mediated.

\section{CONCLUSIONS}

Here we have shown that administration of atorvastatin and pravastatin resulted in accelerated arthritis onset in a mouse CIA model. Atorvastatin administration after $\mathrm{CIA}$ induction resulted in earlier onset than atorvastatin administration before $\mathrm{CIA}$ induction, or than pravastatin administration before or after CIA induction. Atorvastatin administration, especially before CIA induction, resulted in increased autoantibody production. Importantly, our data provide a causal relationship for previous results obtained from observational studies 9,10.

\section{Acknowledgements}

Dr T. Cantaert (Academic Medical Centre/University of Amsterdam) and Dr S. Eussen (National Institute for Public Health and the Environment) are gratefully acknowledged for their expert advice. Prof W. Van Den Berg is acknowledged for critical review of the manuscript. Pfizer is acknowledged for their kind gift of atorvastatin. 


\section{REFERENCES}

1. Baigent C, Keech A, Kearney PM, et al. Efficacy and safety of cholesterol-lowering treatment: prospective meta-analysis of data from 90,056 participants in 14 randomised trials of statins. Lancet 2005; 366(9493): 1267-78.

2. Bellosta S, Paoletti R, Corsini A. Safety of statins: focus on clinical pharmacokinetics and drug interactions. Circulation 2004; 109(23 Suppl 1): III50-7.

3. Colhoun HM, Betteridge DJ, Durrington PN, et al. Primary prevention of cardiovascular disease with atorvastatin in type 2 diabetes in the Collaborative Atorvastatin Diabetes Study (CARDS): multicentre randomised placebo-controlled trial. Lancet 2004; 364(9435): 685-96.

4. Sever PS, Dahlof B, Poulter NR, et al. Prevention of coronary and stroke events with atorvastatin in hypertensive patients who have average or lower-than-average cholesterol concentrations, in the Anglo-Scandinavian Cardiac Outcomes Trial--Lipid Lowering Arm (ASCOT-LLA): a multicentre randomised controlled trial. Lancet 2003; 361(9364): 1149-58.

5. Arnaud C, Braunersreuther V, Mach F. Toward immunomodulatory and anti-inflammatory properties of statins. Trends Cardiovasc Med 2005; 15(6): 202-6.

6. Kwak BR, Mulhaupt F, Mach F. Atherosclerosis: anti-inflammatory and immunomodulatory activities of statins. Autoimmun Rev 2003; 2(6): 332-8.

7. Mausner-Fainberg K, Luboshits G, Mor A, Maysel-Auslender S, Rubinstein A, Keren G, George J. The effect of HMG-CoA reductase inhibitors on naturally occurring CD4+CD25+ T cells. Atherosclerosis 2008; 197(2): 829-39.

8. Ridker PM, Danielson E, Fonseca FA, et al. Reduction in C-reactive protein and LDL cholesterol and cardiovascular event rates after initiation of rosuvastatin: a prospective study of the JUPITER trial. Lancet 2009; 373(9670): 1175-82.

9. De Jong $\mathrm{HJ}$, Klungel $\mathrm{OH}$, Van Dijk L, et al. Use of statins is associated with an increased risk of rheumatoid arthritis. Ann Rheum Dis 2012; 71(5): 648-54

10. De Jong HJ, Tervaert JW, Saldi SR, et al. Association between statin use and lupus-like syndrome using spontaneous reports. Semin Arthritis Rheum 2011; 41(3): 373-81.

11. Moulis G, Béné J, Sommet A, Sailler L, Lapeyre-Mestre M, Montastruc JL, French Association of PharmacoVigilance Centres. Statin-induced lupus: a case/non-case study in a nationwide pharmacovigilance database. Lupus 2012; 21(8): 885-9.

12. Bevaart L, Vervoordeldonk MJ, Tak PP. Collagen-induced arthritis in mice. Methods Mol Biol 2010; 602: 181-92.

13. Ho PP, Higgins JP, Kidd BA, et al. Tolerizing DNA vaccines for autoimmune arthritis. Autoimmunity 2006; 39(8): 675-82.

14. Yamagata T, Kinoshita K, Nozaki Y, Sugiyama M, Ikoma S, Funauchi M. Effects of pravastatin in murine collagen-induced arthritis. Rheumatol Int 2007; 27(7): 631-9.

15. Leung BP, Sattar N, Crilly A, et al. A novel anti-inflammatory role for simvastatin in inflammatory arthritis. J Immunol 2003; 170(3): 1524-30.

16. Palmer G, Chobaz V, Talabot-Ayer D, Taylor S, So A, Gabay C, Busso N. Assessment of the efficacy of different statins in murine collagen-induced arthritis. Arthritis Rheum 2004; 50(12): 4051-9.

17. Zadelaar S, Kleemann R, Verschuren L, De Vries-Van Der Weij J, Van Der Hoorn J, Princen HM, Kooistra T. Mouse models for atherosclerosis and pharmaceutical modifiers. Arterioscler Thromb Vasc Biol 2007; 27(8): 1706-21.

18. Maron DJ, Fazio S, Linton MF. Current perspectives on statins. Circulation 2000; 101(2): 207-13.

19. Vandebriel RJ, Gremmer ER, Vermeulen JP, Hellwig SM, Dormans JA, Roholl PJ, Mooi FR. Lung pathology and immediate hypersensitivity in a mouse model after vaccination with pertussis vaccines and challenge with Bordetella pertussis. Vaccine 2007; 25(12): 2346-60.

20. Slob W. Dose-response modeling of continuous endpoints. Toxicol Sci 2002; 66(2): 298-312

21. PROAST. Avalaible at:http://www.proast.nl.

22. Appel MJ, Bouman HGM, Pieters MN, Slob W. Evaluation of the applicability of the Benchmark approach to existing toxicological data (RIVM report 601930 001). Bilthoven, The Netherlands: RIVM, 2001. 
23. Nielen MM, Van Schaardenburg D, Reesink HW, et al. Specific autoantibodies precede the symptoms of rheumatoid arthritis: a study of serial measurements in blood donors. Arthritis Rheum 2004; 50(2): 380-6.

24. Murphy CA, Langrish CL, Chen Y, et al. Divergent pro- and antiinflammatory roles for IL-23 and IL-12 in joint autoimmune inflammation. J Exp Med 2003; 198(12): 1951-7.

25. Lubberts E, Joosten LA, Oppers B, et al. IL-1-independent role of IL-17 in synovial inflammation and joint destruction during collagen-induced arthritis. J Immunol 2001; 167(2): 1004-13.

26. Nakae S, Nambu A, Sudo K, Iwakura Y. Suppression of immune induction of collagen-induced arthritis in IL-17-deficient mice. J Immunol 2003; 171(11): 6173-7.

27. Lubberts E, Koenders MI, Oppers-Walgreen B, Van Den Bersselaar L, Coenen-De Roo CJ, Joosten LA, Van Den Berg WB. Treatment with a neutralizing anti-murine interleukin-17 antibody after the onset of collagen-induced arthritis reduces joint inflammation, cartilage destruction, and bone erosion. Arthritis Rheum 2004; 50 (2): 650-9

28. Schurgers E, Billiau A, Matthys P. Collagen-induced arthritis as an animal model for rheumatoid arthritis: focus on interferon-gamma. J Interferon Cytokine Res 2011; 31(12): 917-26.

29. Youssef S, Stüve O, Patarroyo JC, et al. The HMG-CoA reductase inhibitor, atorvastatin, promotes a Th2 bias and reverses paralysis in central nervous system autoimmune disease. Nature 2002; 420(6911): 78-84.

30. Manoury-Schwartz B, Chiocchia G, Bessis N, et al. High susceptibility to collagen-induced arthritis in mice lacking IFN-gamma receptors. J Immunol 1997; 158(11): 5501-6.

31. Vermeire K, Heremans H, Vandeputte M, Huang S, Billiau A, Matthys P. Accelerated collagen-induced arthritis in IFN-gamma receptor-deficient mice. J Immunol 1997; 158(11): 5507-13.

32. Coward WR, Marei A, Yang A, Vasa-Nicotera MM, Chow SC. Statin-induced proinflammatory response in mitogen-activated peripheral blood mononuclear cells through the activation of caspase-1 and IL-18 secretion in monocytes. J Immunol 2006; 176(9): 5284-92.

33. Wannamaker W, Davies R, Namchuk M, et al. (S)-1-((S)-2-\{[1-(4-amino-3-chloro-phenyl)-methanoyl]amino\}-3,3-dimethyl-butanoy I)-pyrrolidine-2-carboxylic acid ((2R,3S)-2-ethoxy-5-oxo-tetrahydro-furan3-yl)-amide (VX-765), an orally available selective interleukin (IL)-converting enzyme/caspase-1 inhibitor, exhibits potent anti-inflammatory activities by inhibiting the release of IL-1beta and IL-18. J Pharmacol Exp Ther 2007; 321(2): 509-16.

34. Hom JT, Bendele AM, Carlson DG. In vivo administration with IL-1 accelerates the development of collagen-induced arthritis in mice. J Immunol 1988; 141(3): 834-41.

35. Joosten LA, Helsen MM, Van De Loo FA, Van Den Berg WB. Anticytokine treatment of established type II collagen-induced arthritis in DBA/1 mice. A comparative study using anti-TNF alpha, anti-IL-1 alpha/ beta, and IL-1Ra. Arthritis Rheum 1996; 39(5): 797-809.

36. Gracie JA, Forsey RJ, Chan WL, et al. A proinflammatory role for IL-18 in rheumatoid arthritis. J Clin Invest 1999; 104(10): 1393-401.

37. Leung BP, McInnes IB, Esfandiari E, Wei XQ, Liew FY. Combined effects of IL-12 and IL-18 on the induction of collagen-induced arthritis. J Immunol 2000; 164(12): 6495-502.

38. Wei XQ, Leung BP, Arthur HM, McInnes IB, Liew FY. Reduced incidence and severity of collagen-induced arthritis in mice lacking IL-18. J Immunol 2001; 166(1): 517-21.

39. Bendele A. Animal models of rheumatoid arthritis. J Musculoskelet Neuronal Interact 2001; 1(4): 377-85.

40. Brand DD. Rodent models of rheumatoid arthritis. Comp Med 2005; 55(2): 114-22.

41. Hegen M, Keith JC, Jr, Collins M, Nickerson-Nutter CL. Utility of animal models for identification of potential therapeutics for rheumatoid arthritis. Ann Rheum Dis 2008; 67(11): 1505-15.

42. Kollias G, Papadaki P, Apparailly F, et al. Animal models for arthritis: innovative tools for prevention and treatment. Ann Rheum Dis 2011; 70(8): 1357-62. 


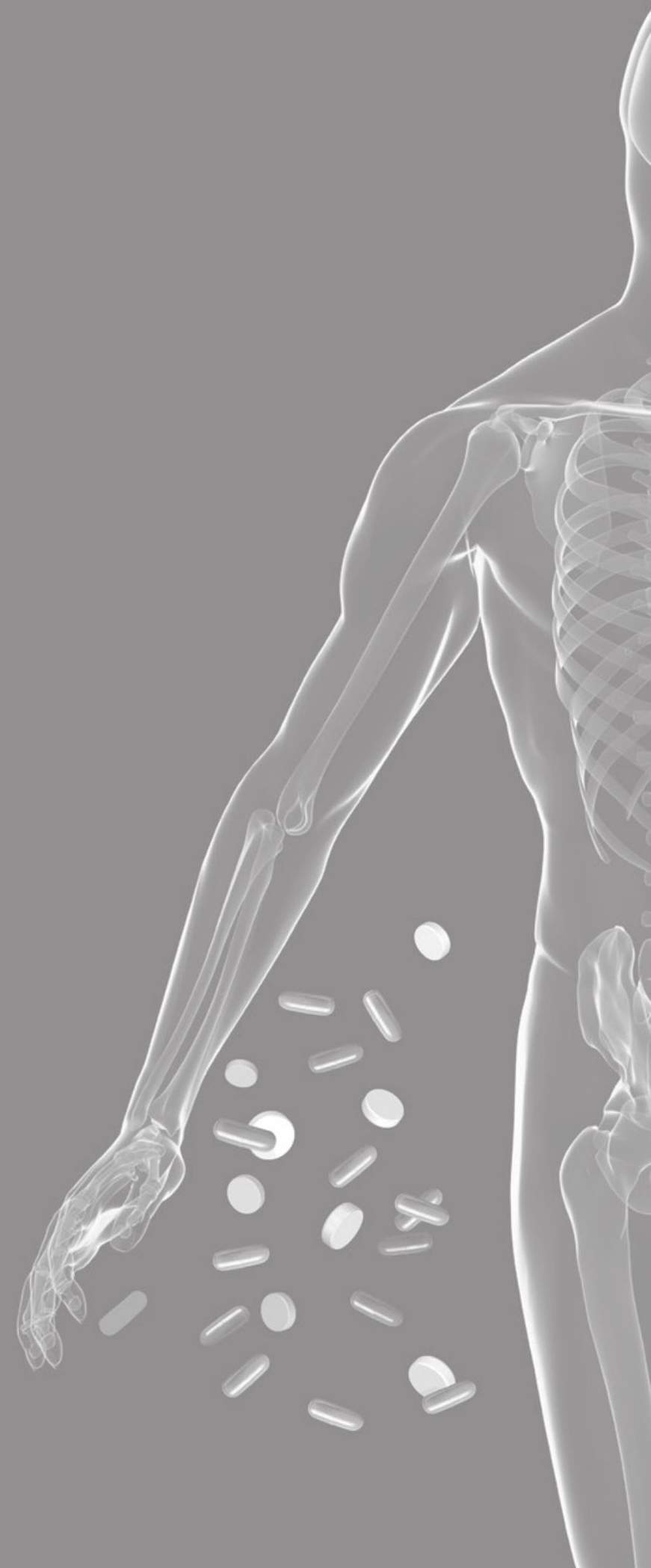




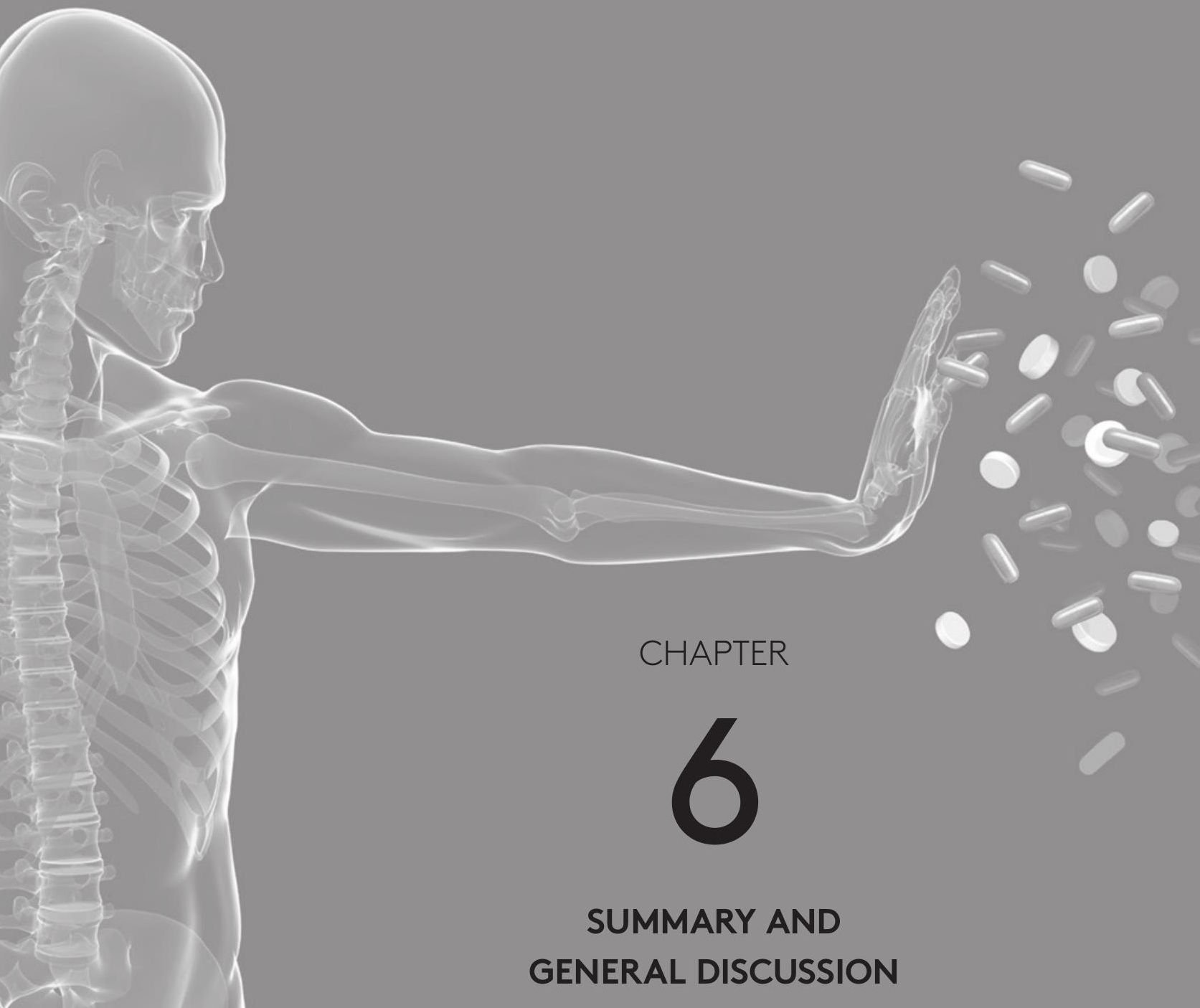





\section{INTRODUCTION}

In recent decades, it has been recognised that the development of autoimmunity is a multi-factorial process in which both genetic and environmental features interfere and contribute to the aetiology and progression of the disease. Despite many years of intensive research, exact mechanisms of initiation of autoimmunity remain unclear. Several studies have demonstrated that environmental components play a major role in this process of autoimmunity ${ }^{1-5}$ and may be responsible for the increased prevalence of autoimmune disorders in highly industrialised countries 6 . Many drugs are associated with the development of certain autoimmune disorders, e.g., lupus-like syndrome and vasculitis $7-19$

Statins, angiotensin converting enzyme (ACE) inhibitors and angiotensin receptor blockers (ARBs) are widely prescribed to reduce the risk of cardiovascular morbidity and mortality in patients with or at risk for coronary heart disease 20-25. In addition to their cholesterol and blood pressure lowering effects, several studies have shown that these drugs also have anti-inflammatory and immunomodulatory properties 26-43, and are thereby also effective in the treatment of certain autoimmune disorders. The beneficial effects of these drugs are well described, but interestingly, such immunomodulating effects may, on the other hand, adversely influence immune regulation. Therefore, a hypothesis may be put forward that statins, ACE inhibitors and ARBs facilitate the development of autoimmunity, potentially resulting in autoimmune disorders.

At the time of marketing authorisation of a drug, not all is known about the safety of a drug. The full safety profile of a drug may not be known until many years after its market launch. This knowledge gap may be due to limitations in clinical trials, including homogeneous populations, small sample size, limited duration and the inability to predict the real world ${ }^{44}$. Post-marketing collected safety data offers a valuable and necessary addition to clinical trials ${ }^{44}$, as is shown in this thesis. Currently, a more pro-active approach towards the identification and quantification of safety data has been anticipated 45,46. Actually, among regulators and pharmaceutical industries there is an agreement that safety data should be continuously collected throughout its market life 47 . This agreement has been implemented in several guidelines for risk management programs 48,49 . Not only the pro-active approach by pharmaceutical industries and regulatory authorities, but also the routine approaches in pharmacovigilance, such as spontaneous reporting of adverse drug reactions (ADRs) by healthcare professionals, patients, regulatory authorities and pharmaceutical industries are of importance in the detection of new, rare and/or serious ADRs 50 . In clinical practice, it is a challenge to detect autoimmune disorder-associated ADRs as they are relatively uncommon, appear after long-term use and may not disappear after 
withdrawal of these drugs $51-53$. This thesis aims to explore whether there is an association between the use of cardiovascular drugs (statins, ACE inhibitors and ARBs) and the incidence of autoimmune disorders. In this chapter, we summarise and discuss our main findings and immune-mediated mechanisms of statin-associated immune disorders, consider several methodological issues, address the practical and clinical implications, and present important areas for future research.

\section{SUMMARY OF THE MAIN FINDINGS}

\section{Signal detection for statin-associated autoimmune disorders in spontaneous reporting databases}

Since the introduction of statins to the market in the late eighties ${ }^{54}$, increasing numbers of cases of statin-associated lupus-like syndrome, vasculitis and polymyalgia rheumatica (PMR) have been reported in the literature 55-61. Based on these case reports, we assessed whether there was an association between statin use and the occurrence of lupus-like syndrome (chapter 2.1), and PMR (chapter 2.2), respectively. In the first study, using a spontaneous reporting database (VigiBase), we found that statin use was more often reported in patients with lupus-like syndrome than in patients who experienced other ADRs (chapter 2.1). In accordance with our finding, Moulis et al. observed an association between statin use and reporting lupus erythematosus in the French PharmacoVigilance database ${ }^{62}$. Our second study showed a strong association between statin use and reporting of PMR in a spontaneous reporting database (chapter 2.2). Furthermore, in this study, six individual case safety reports (ICRs) had records of recurrence of PMR after re-exposure to statins, which strengthened the suspicion regarding the drug's causal involvement in these patients. The results of both studies lend support to previous anecdotal case reports in the literature ${ }^{57-59}$, suggesting a pharmacovigilance signal of statin-associated lupus-like syndrome and PMR.

To strengthen our findings of statin-associated PMR, we described a patient in more detail to illustrate the possible causality of statin-associated PMR/giant cell arteritis (GCA). In this case report, we describe the disappearance of PMR after withdrawal of statins and recurrence of PMR/GCA after resumption of treatment with a statin, which is strongly suggestive of statin-induced PMR/GCA (chapter 2.3).

\section{Testing the association between cardiovascular drug use and the incidence of autoimmune disorders in electronic health record databases}

As the spontaneous reporting database is primarily used for signal detection (hypothesis strengthening) and hypothesis generation purposes, and not for hypothesis testing, 
we tested our hypothesis in electronic health record databases. As described in chapter 3, we performed two studies to determine whether statin use was associated with an increased risk of developing RA. In the first study, statin use and incident RA were derived from data on drug prescriptions, morbidity and referrals of the Netherlands Information Network of General Practice (LINH). By performing a casecontrol study, we found an increased risk of developing RA within six months of statin use (chapter 3.1). A consistent trend of increasing risk of developing RA with increased duration, defined daily doses and number of prescriptions, was not observed, nor with adherence to statin treatment. However, we observed a small trend between increasing potencies of statin treatment and the risk of RA. To deal with the limitations of a case-control study 63 , we performed a second study where we addressed the same research question by using a time-dependent analysis. We have confirmed and extended our findings by describing the patterns of risks of RA (hazard rates) with changes in statin exposure over time in a large retrospective cohort study, the United Kingdom (UK) Clinical Practice Research Datalink (CPRD). In this second study, statin use and incident RA were derived from data on prescriptions, diagnoses and referrals to specialist care. For the second time, we found that statin use was associated with an increased risk of developing RA (chapter 3.2). The risk of RA was substantially increased in the first year after the start of exposure to statins and then diminished to baseline. The risk of RA was highest among women and restart users. Since the risk of developing RA was increased shortly after statin initiation, it is possible that statins can accelerate RA onset in patients susceptible to developing RA, as was demonstrated by our in vivo study which is discussed later in this chapter. In a systematic review of case reports of statin-associated lupus-like syndrome, it was shown that the majority of these cases also had a time to onset of less than a year 60 . However, the use of statins for a prolonged time is probably safe and well tolerated in patients not prone to develop RA. This could also explain the difference in the risk of developing RA in 'de novo' and 'restart' users. It may be that 'de novo' users were patients who can tolerate statins whereas 'restart' users were patients who were less likely to be fully adherent to statins because of potential discomfort or side effects. Another explanation could be our observed effect modification by sex: men were not at risk whereas women were at high risk for developing RA. Accordingly, it has been shown that women are more prone to developing RA64,65. To date, five other studies have assessed the risk of incident RA after statin treatment, and have shown conflicting results 66-70. None of these studies found an increased risk of developing RA with statin use. Three population-based cohort studies reported that statins may exert a protective effect on the development of RA $66,67,70$ whereas two other population-based cohort studies did not observe an effect 68,69 . These findings conflict with our studies which may be explained by considering a lag-time between exposure to statins and incident RA 68,69 , using different definitions of RA $67-70$ or 
exposure to statins $66-70$, comparing to a control group of non-persistent statin users instead of non-users 67 , controlling for other confounders $66-70$, shifting the date of the first event of RA 66, conducting separate analyses in patients with or without a medical history of cardiovascular risk factors 66 , or propensity score matching on baseline characteristics 70 (Table 1). This latter method was originally proposed by Rosenbaum and Rubin in $1983^{71}$. With this method, a subject's propensity score is defined as the conditional probability of receiving a certain treatment (versus another), given a collection of observed confounders. In general, this probability is unknown, but is usually estimated from the data using logistic regression, modelling exposure status (e.g. exposed or non-exposed to statins) as the dependent variable and the potential confounders as the independent variables. It has been shown that two subjects with the same propensity score have an equal estimated probability of exposure. After matching on the propensity score, the group of subjects who are exposed or non-exposed to statins tend to have the same distribution of potential confounding factors. Balance of the potential confounders across exposed and non-exposed groups can be quantified as proposed by Belitser et al. 72 .

As we have shown an increased risk of developing RA within a year after statin initiation, a plausible explanation for the discrepancy between our findings and the other five observational studies 66-70, may be that our study did not take a lag-time for developing RA into consideration. Although we used two different study designs and databases to study whether statin use was associated with RA, the distribution of cardiovascular diseases and related risk factors (e.g. hyperlipidaemia) were not balanced at baseline in both studies, and may have influenced our results.

We further tested our main hypothesis by performing a study where we investigated whether the use of ACE inhibitors or ARBs is associated with incident RA. In this case-control study, we included only users of ACE inhibitors or ARBs or other antihypertensive drugs (the reference group) (chapter 3.3). Within the Dutch general practice database (LINH), we did not observe an association between ACE inhibitor or ARB use and the risk of developing RA. The effect was consistent when we defined the exposure to ACE inhibitors and ARBs as current and past use, duration, or dosage of use. These results do not support the hypothesis that ACE inhibitors or ARBs either facilitate or protect against RA. To our knowledge, apart from our study, no observational study has investigated the influence of ACE inhibitor or ARB use and incident RA. Evidence for the association between ACE inhibitor or ARB use and the occurrence of autoimmune disorders was derived from case reports, animal models and patients. These findings were, however, inconclusive. Case reports suggested ACE inhibitor or ARB-associated lupus-like syndrome, vasculitis and pemphigus ${ }^{73-94}$, whereas in animal models of induced arthritis, beneficial effects of ACE inhibitor and ARB use were demonstrated ${ }^{95-98}$. Conflicting results were presented in open-label 
studies with patients with RA treated with ACE inhibitors or ARBs. Two open-label studies showed that patients with RA treated with these drugs had reductions in clinical symptoms and inflammation markers 32,33 , whereas in two other studies no clinical improvements were observed 99,100. These latter two studies are in line with our findings. However, these studies were conducted in patients in whom RA had already been diagnosed. In our study, we evaluated the risk of developing RA among patients initially free from RA who were treated with ACE inhibitors or ARBs. Nevertheless, hypertension may be considered as an immune-mediated disease 101 and is more prevalent in patients with RA than in the general population ${ }^{102}$. With regard to their immunomodulatory abilities and the high occurrence of hypertension in patients with RA, ACE inhibitors and ARBs are effective in treating hypertension, and may be beneficial in the treatment of RA.

\section{Statin use and autoimmunity}

In the introduction of this chapter, we postulate that statins may facilitate the development of autoimmunity. It has been suggested that statins, apart from their anti-inflammatory effects, may have a direct immunomodulating effect on $T$ cells and may promote a shift from Thelper (Th) 1 to Th2 immune responses, possibly leading to a dysregulation in the immune homeostasis $36,60,103$. In the studies above, we were not able to evaluate this hypothesis, but by using serum samples from the Doetinchem Cohort Study which was linked to PHARMO Record Linkage System (PHARMO-RLS), a database comprising pharmacy-dispending records on statin use, we could study the association between statin use and markers of (auto) immunity. We studied the effects of statin use on markers of innate and adaptive immunity, and self-tolerance by measuring serum levels of $\mathrm{C}$-reactive protein (CRP), neopterin, immunoglobulin $\mathrm{E}$ ( $\operatorname{lgE}$ ) antibodies and the presence of autoantibodies (antinuclear antibodies (ANA) and IgM rheumatoid factor (RF)) in subjects from the general population who were either statin user or non-user (chapter 4). As also observed in population-based studies and clinical trials 104-109, levels of CRP were found to be lower in the statin users than in non-users. By confirming this well-known association between statin use and decreased levels of CRP, we have shown that we have used appropriate methods for patient selection in our study. In addition, we found in statin users a trend towards higher levels of IgE antibodies, a surrogate marker for Th2 response. No differences were observed in the levels of neopterin, a surrogate marker for Th1 response, and/or the presence or absence of autoantibodies between statin users and non-users. These findings suggest that statins exert anti-inflammatory effects but do not provide evidence that statins exert immunomodulatory effects on a Th1 response and/or loss of self-tolerance. However, an immunomodulatory effect on Th2 response cannot be excluded. This latter finding should be investigated in peripheral blood of healthy individuals instead of sera. Our finding on Th2 response 
Table 1 All observational studies showing the risk of developing rheumatoid arthritis with statin use

\section{Observational studies of statin-associated RA}

\begin{tabular}{|c|c|}
\hline Data source & $\begin{array}{l}\text { UK Health Improvement Network } \\
\text { Database (THIN) }\end{array}$ \\
\hline Study design & Retrospective cohort \\
\hline Study population & $\begin{array}{l}\text { All patients registered with a GP } \\
\text { between Jan } 1995 \text { - Dec } 2006 \\
\text { Comprising EMR for } 5.5 \text { million } \\
\text { patients derived from } 303 \\
\text { practices }\end{array}$ \\
\hline
\end{tabular}

\section{Smeeth et al., 200869}

UK Health Improvement Network

(THIN)

All patients registered with a GP between Jan 1995 - Dec 2006 patients derived from 303 practices
Jick et al., 200966

General Practice Research

Database (GPRD)

Nested case-control

All patients registered with a GP between Jan 1, 1992 - Dec 31, 2001

Since 1987, $\geq 5$ million residents in the UK have been enrolled
Exposure to statins

Control group

Medical history
All patients aged $40-80$ years who received their first prescription for a statin on or after Jan 1, 1995 Date of the first prescription of statins is defined as index date
- All patients aged $40-89$ years with at least 1 prescription for a statin at any time

Current statin use was defined as receipt of at least two prescriptions within 1 year preceding the firsttime diagnosis of RA

All other receipt of statins was considered past use

Randomly selected up to 4 controls, i.e. people without a diagnosis of RA

Matched by age (within 1 year), sex, calendar time (using the same date as first-time diagnosis of RA), practice and number of years of previous recorded history

At least one year before start statin treatment

First time diagnosis of RA Included cases with a stated diagnosis of RA and/or a referral to outpatient specialist or inpatient hospitalization and use of DMARDs 
Hippisley-Cox et al., 201068

QResearch Database

Prospective cohort

All practices in England and

Wales using the computer based Egton Medical Information System (EMIS)

All patients registered with the practices between Jan 1, 2002 June 30, 2008
Chodick et al., 201067

Maccabi Healthcare Service

(MHS)

Retrospective cohort

All patients who were continuously enrolled in the Israel Health Maintenance Organisation (HMO) from 1995-1998 were included in the cohort (Jan 1, 1998 - July 1 2007)

Comprising 1.8 million people in the $\mathrm{HMO}$

All patients aged $\geq 18$ years who had at least 1 dispensed prescription of statins during the study period

Date of the first dispensed statin is defined as index date
Schmidt et al., 201370

San Antonio area military health care system

Retrospective cohort

All patients who were enrolled as Tricare Prime or Tricare Plus in the San Antonio are military health care system between Oct 1, 2003- Mar 5, 2010

Study period was divided into baseline period (Oct 1, 2003Sept 30, 2005) for describing the baseline characteristics and follow-up period (Oct 1 , 2005-Mar 5, 2010) for identifying connective tissue diseases

All patients aged $30-85$ years who received a statin prescription of at least 90-day supply between Oct 1, 2004Sept 30, 2005

\author{
Non-use of statins during the \\ study period \\ $<20 \%$ proportion of days \\ covered with statins
}

Did not receive a statin at any time during the study

\begin{abstract}
At least one year registered with the general practice when the date of the study started

Patients who did not receive a statin prescription at least 3 year prior to the index date
\end{abstract}

First time diagnosis of RA
First time diagnosis of RA
At least one year before start statin treatment (start date baseline period Oct 1, 2003 and start date statin prescription Oct 1, 2004)

The occurrence of 3 separate codes for connective tissue disorder, including RA, SLE, dermatomyositis, polymyositis, polymyalgia rheumatica, sicca syndrome, keratoconjuctivitis sicca, Sjögren's disease and connective tissue disorder (unspecified) during the followup period 


\title{
Table 1 Continued
}

\author{
Observational studies Smeeth et al., $200869 \quad$ Jick et al., 200966 \\ of statin-associated RA \\ Shifting the date of the \\ first diagnosis of RA \\ Considering a lag-time \\ $-$ \\ Exactly one year prior to the date of \\ the first RA diagnosis (index date) \\ Time-dependent \\ analysis \\ - Cases of RA in the first year after \\ statin initiation were excluded
}

\section{Confounders}

year initiation of statin treatment,
first diagnosis of the following
post-index date: diabetes,
cerebrovascular disease,
coronary heart disease, peripher
vascular disease, other atheroma,
atrial fibrillation, heart failure,
hyperlipidaemia, hypertension,
other circulatory disease, cancer
dementia, first use of the followin
post-index date: aspririn, nitrates
fibrates, $\beta$-blockers, CCB,
potassium channel activators,
diuretics, positive inotropes,
anticoagulants, anti-hypertensive
drugs, or other cardiovascular
drugs
. Adjusted for propensity score*
Propensity score is based on
factors that may influence statin
prescribing

Subgroup analysis

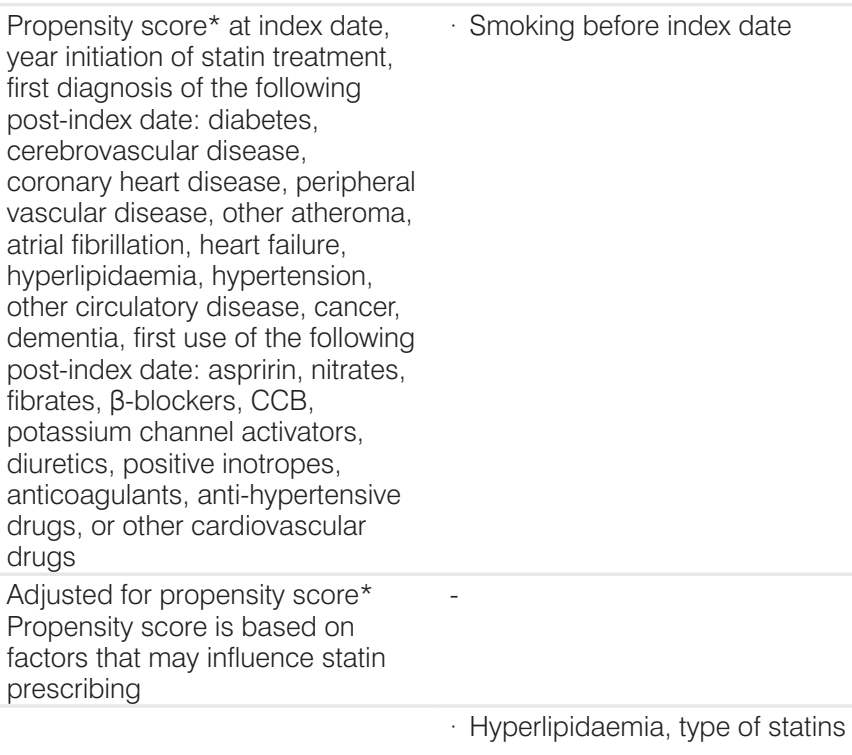

RA, rheumatoid arthritis; GP, general practitioner; EMR, electronical medical records; UK, United Kingdom; DMARDs, disease-modifying anti-rheumatoid drugs; BMI, body mass index; CCB, calcium channel blockers; LDL, low-density lipoprotein

*included covariates in the propensity score model: body mass index, socioeconomic status, consultation rate, prescribing rate, smoking status, drinking habits, diabetes, coronary heart disease, cerebrovascular disease, peripheral vascular disease, other atheroma, other circulatory disease, dementia, cancer, atrial fibrillation, heart failure, recent hepatic disease, recent renal disease, thyroid disease, hyperlipidaemia, hypertension, recent use of hormone replacement therapy, anti-psychotics, antidepressants, steroids (oral or inhaled), fibrates, cytochrome P450 3A4 inhibitors, any prior use of non-statin or fibrate lipid-lowering medication, nitrates, aspirin, $\beta$-blockers, calcium channel blockers, potassium channel activators, diuretics, positive inotropes, anticoagulants, anti-hypertensive drugs, or other cardiovascular drugs. 
Hippisley-Cox et al., 201068

Chodick et al., 201067

Schmidt et al., 201370

\begin{tabular}{|c|c|c|}
\hline- & - & - \\
\hline- & $\begin{array}{l}\text { Cases of RA in the first year after } \\
\text { statin initiation were excluded }\end{array}$ & - \\
\hline $\begin{array}{l}\text { To determine the risk of RA within } \\
1 \text { year, } 1-3 \text { years, } 3-5 \text { years } \\
\text { and } \geq 5 \text { years of taking statins }\end{array}$ & - & - \\
\hline $\begin{array}{l}\text { Age, BMI, ethnicity, smoking, } \\
\text { hypothyroidism at start study }\end{array}$ & $\begin{array}{l}\text { Age, sex, socioeconomic level, } \\
\text { utilization of healthcare services } \\
\text { in the year before the index date }\end{array}$ & $\begin{array}{l}\text { In the propensity score matched } \\
\text { cohort: no correction for } \\
\text { confounders } \\
\text { In the unmatched cohort: } \\
\text { adjusted for all covariates which } \\
\text { were included in the propensity } \\
\text { score model }\end{array}$ \\
\hline
\end{tabular}

Matching on propensity score**

Sex, type of statins

\begin{abstract}
Age categories, sex, baseline
LDL levels and efficacy of initial

statin therapy
\end{abstract}

**included covariates in the propensity score model: age, sex, acute myocardial infarction, congestive heart failure, peripheral vascular disease, cerebrovascular disease, dementia, chronic obstructive pulmonary diseases, rheumatologic diseases, peptic ulcer disease, mild liver disease, diabetes mellitus, diabetes mellitus complications, hemiplegia/paraplegia, renal disease, malignancy, liver disease (moderate/severe), metastatic neoplasm, HIV, illicit drug use, alcohol abuse/dependence, smoker, total Charlson co-morbidity index, number of outpatient medical encounters and inpatient admissions during each of the baseline period and the follow-up period, and the use of $\beta$-blockers, calcium channel blockers, diuretics, non-statin lipidlowering drugs, angiotensin receptor blockers/ angiotensin converting enzyme inhibitors, oral hypoglycemic, cytochrome P450, aspirin, non-steroidal anti-inflammatory drugs, selective serotonin reuptake inhibitors, systemic corticosteroids, anti-psychotics, sedatives, tricyclic antidepressants. 
is in line with the results of two other studies which suggested that statins promote a Th2 bias of the immune response 110,111. In contrast to this finding, Cherfan et al. reported that statins suppress Th2 response 107. As for the findings on Th1 response, our findings are in agreement with three other studies that found no effect on the levels of neopterin after treatment with statins 107,112,113, whereas in two other studies neopterin levels declined during statin treatment 114,115. To date, no studies have compared the presence of autoantibodies between statin users and non-users in the general population. As we did not observe an effect of statin use on the presence of autoantibodies in the general population, it seems that statins do not themselves cause a breakdown of self-tolerance, but may promote a pre-existing autoimmune-prone condition to develop towards a clinically manifest disease. However, in our animal study only administration of a specific type of statin, atorvastatin, before induction of collagen-induced arthritis has resulted in an increased autoantibody production (chapter 5).

\section{Causal relationship between statin use and autoimmune disorders}

Based on the findings presented in chapter 3 , it has been proposed that statin use is associated with an increased risk of developing RA. Yet, other observational studies found that statins have a protective effect, or no effect on developing RA 66-70. To date, our findings have not been corroborated by other studies. To further evaluate the association between statin use and the risk of developing RA, we performed an in vivo study, in a murine experimental model for RA, to investigate whether a causal relationship between statin use and the risk of developing RA could be established (chapter 5). In this study, we used the murine collagen type II (CII)-induced arthritis (CIA) model which is a well-known animal model for RA. In this animal model, mice are immunised by an injection of an emulsion of bovine Cll mixed and Freund's complete adjuvant, and are challenged three weeks later with $\mathrm{Cll}$ alone, producing an immune response to connective tissues. Arthritis is scored from the time of challenge onwards 116. The CIA mice were orally administered with pravastatin, atorvastatin or phosphate buffered saline (PBS) daily for four to six weeks, either before (day -28 until day 20) or after (day 22 until day 42) CIA induction ( $n=12$ per group). In addition, we included unchallenged mice that only received PBS. We found that treatment with both $40 \mathrm{mg} / \mathrm{kg}$ pravastatin and $10 \mathrm{mg} / \mathrm{kg}$ atorvastation, either before or after $\mathrm{ClA}$ induction resulted in earlier arthritis onset compared to no treatment. Moreover, we observed that atorvastatin and pravastatin administration after CIA induction resulted in earlier arthritis onset than administration with the same statins before CIA induction. The observation of earlier arthritis onset after treatment with statins before CIA induction supports the results from chapter 3.1 and chapter 3.2. Four other animal studies, using the CIA murine model, have shown beneficial effects or no effects of statin treatment on arthritis onset 40,117-119. All of these of studies used different types, 
routes, doses per body weight $(\mathrm{mg} / \mathrm{kg}$ ), initiation and duration of statin exposure. This substantial heterogeneity in approach and methodology between these studies, including ours, makes it difficult to compare the results from all these studies. Nevertheless, our study supports a causal relationship between statin use and the risk of developing RA. The murine model we have used in this study, however, seems to be an appropriate model for testing statin exposure prior to expression of arthritis.

\section{IMMUNE-MEDIATED MECHANISMS OF STATIN- ASSOCIATED IMMUNE DISORDERS}

In this thesis we have assessed a causal relationship between statin use and the risk of developing RA. However, our data did not provide a possible mechanism for the statin-associated RA, nor did we reveal the mechanism of statin-associated lupus-like syndrome or PMR.

It has been suggested that statins may promote a shift in Th1/Th2 balance 36,60 , affect regulatory T cells ${ }^{120-122}$, or lead to unstable regulatory $T$ cells in the periphery. All of these mechanisms may promote autoimmunity ${ }^{123,124}$. In addition, statins may exacerbate or trigger cellular apoptosis 60,125,126, which may result in increased amounts of autoantigens in the circulation 127,128 due to less efficient clearing of apoptotic cells. Also, statins may induce neo-antigens as a result of muscle damage which are subsequently presented to the immune system, as observed in statin-associated necrotising myopathy 129,130. A similar mechanism may be operative in statin-associated PMR. Furthermore, statins may inhibit the prenylation of Rho GTPases, including Rac-1 131,132. Rac-1 is directly implicated in the activation of caspase-1 133, which is crucial for processing and secretion of the pro-inflammatory cytokines interleukin (IL)-1 $1 \beta$ and IL-18 and to a lesser extent of IL-12, an inducer of IL-18, thereby promoting autoimmunity ${ }^{134-136}$. Statins may be able to amplify autoimmunity in genetically susceptible individuals, thereby increasing the risk of developing autoimmune disorders ${ }^{137}$. Another possible hypothesis is that the self-tolerance is lost due to persistence of infectious agents in individuals who were treated with statins. Since statins may reduce Th1 responses ${ }^{42}$, infectious agents may not be cleared as efficiently as under normal circumstances ${ }^{138}$, or statins themselves may increase the risk of herpes zoster 139,140 or the commonly encountered infections (e.g. sepsis) ${ }^{141}$. As a consequence, these individuals may become immunocompromised. Individuals who are immunocompromised may not have the ability to respond normally to infections due to an impaired or weakened immune system, possibly resulting in a loss of self-tolerance. 


\section{METHODOLOGICAL CONSIDERATIONS}

\section{Type of study design}

Different types of study design have been used in chapters 2, 3, 4 and 5 to address the association between prolonged use of cardiovascular drugs, statins, ACE inhibitors and ARBs, and the incidence of autoimmunity/autoimmune disorders. The differences in study design have been depicted in Figure 1. In this section, we will discuss the rationale for using different study designs and their strengths and limitations.

Descriptions of statin-induced autoimmune disorders are sparsely found in the literature. To date, only case reports have been reported and reviewed. Case reports provide a first opportunity to discover and explore potential associations 142. Qualitative signal detection on possible causal relations between an adverse event and a drug can then be detected through case assessment 143,144 as was performed by Moulis et al. using the French PharmacoVigilance database ${ }^{62}$. For causality assessment, several decision algorithms have been introduced 145 which require positive dechallenge (disappearance of the adverse reaction after withdrawal of the drug) or positive rechallenge (recurrence of the adverse event after re-exposure) to fulfil the criteria of 'certain causality' 143 . In chapter 2.3 we describe a case with a positive rechallenge of statin-associated PMR.

When substantial numbers of case reports are available as in the spontaneous reporting system from the WHO collaborating Centre for International Drug Monitoring, comparing the proportion of these reports of adverse events with a specific type of drug may provide strong hypothesis-generating signals. The studies described in chapter 2.1 and 2.2 were conducted as a first essential step to strengthen our hypothesis of an association between statin use and the incidence of autoimmune disorders. Data from spontaneous reporting systems offer the opportunity to monitor all drugs after marketing. In addition, studies using these data may generate alerts and are not limit to the monitoring of specific patient groups. On the other hand, these ICRs have been submitted by governmental pharmacovigilance centres around the world and are heterogeneous in their source, documentation and relationship likelihood ${ }^{146}$. A registry of this kind is not complete and reporting bias may occur as a result of differential underreporting ${ }^{147-149}$. By including reports in which lupus-like syndrome or PMR were related to the suspected or concomitant drug (statins), we have tried to reduce the problem of selective reporting. The advantage of this analysis is that, the case/ non-case selection is neutral for exposure to statins ${ }^{149}$. Nonetheless, case-by-case analysis is a valuable tool in pharmacovigilance and drug safety research 147 . 


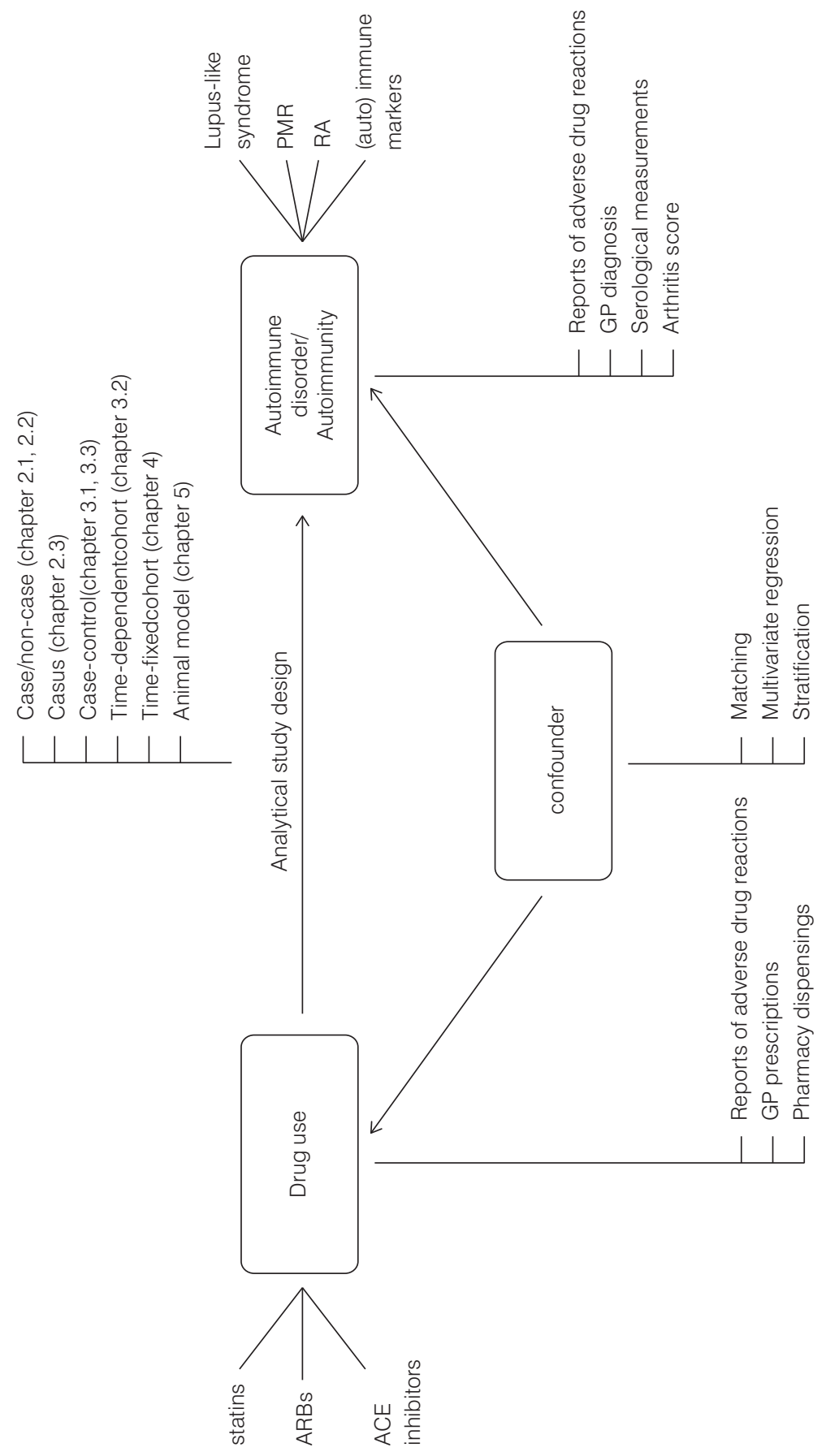

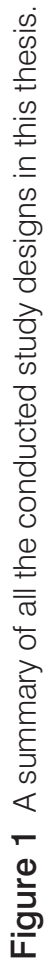


As we detected a signal as reported in chapter 2, we tested our hypothesis in observational studies. The results from observational studies on the risk of autoimmune disorders associated with statins are scarce and lack consistency. Observational studies play an important role in establishing the safety profile of statin use in the post-marketing phase. Observational studies are valuable to test hypotheses and quantify the size of the effect of statins (chapters 3.1 and 3.2), ACE inhibitors, or ARBs (chapter 3.3) on the risk of developing RA. With regard to selecting a study design for assessing the associations between cardiovascular drugs and the risk of developing RA, no paradigm method exists. A case-control approach enables us to study the rare, potentially adverse events with a delayed outcome (e.g. RA) (chapters 3.1 and 3.3), while follow-up studies in electronic health databases with sufficient power are a good alternative (chapter 3.2).

To test our hypotheses, we used data from GPs in the Netherlands and the UK as these two datasets provide medical information from patients in a real-life setting. Since the data in the GP datasets have already been collected, observational studies can be carried out relatively easily and rapidly compared with randomised controlled trials (RCTs) or animal studies. Furthermore, if exposure data are linked to the outcome of the study, the study can be performed without requiring additional information from patients or physicians.

In the Netherlands and the UK, the vast majority of the population is registered with a GP who acts as a gatekeeper for access to specialised care. The GP is, in general, the first professional to be consulted for diagnoses of health problems or prescription of treatments 150,151 . The data that was continuously recorded in both databases included demographic information, diagnoses, prescription details and details of referrals to specialist care 152,153. However, no data were available on dietary intake and physical activity. In the UK CPRD only limited data were available on smoking and other clinical examinations (e.g. lipid levels, inflammatory and immunomodulatory markers (e.g. CRP, ANA, anti-citrullinated protein antibodies (anti-CCP)), blood pressure, BMI and glucose levels), all of which may be important confounders. When the GP databases are linked and extended with other types of data we may obtain a complete medical history of the patient, thereby improving the quality and quantity of confounding factors. By eliminating confounding as much as possible within each study, the higher its internal validity will be.

We did not have medical information from these patients that were referred to a rheumatologist, such as which treatment the patients were advised to start. However, Dutch guidelines for optimising GP-medical specialist communication instruct medical specialists to inform GPs of the results of the diagnostic tests and treatment advice given to the referred patient 154,155 .

Since potential unmeasured differences in the cardiovascular risk profile and other individuals' characteristics between RA and controls, or statin users and non-users 
may have been present, all three observational studies that we performed may have been subject to residual confounding (i.e. the distortion that remains after controlling for confounding factors in the design and/or analysis of the study) 156.

It has been suggested that statins may dysregulate immune homeostasis and this can result in breakdown of self-tolerance, and induction of autoimmunity. To test this hypothesis (chapter 4), we performed a study using serum samples from the ongoing Dutch Doetinchem Cohort study linked to automated pharmacy-dispending records. With these serum samples, we measured surrogate markers for Th1 and Th2 responses and for loss of self-tolerance, and the well-known marker for inflammation, CRP. To date, in the Doetinchem Cohort study, participants have been re-invited three times for examinations at 5-year intervals. At every examination round, serum was collected from participants, which allows us to study the association between statin use and autoimmunity not only cross-sectionally but also longitudinally. Not only serum, but also anthropometric and blood pressure measurements, and demographic, lifestyle and health characteristics were collected using a self-administered questionnaire. Using these data, we have a detailed description of the participants in a real-life setting.

As described earlier, the observational Doetinchem Cohort study may be subject to residual confounding. However, we have matched the statin users and non-users on several factors; we believe we have reduced residual confounding as much as possible.

All of these observational studies (chapters 3.1, 3.2, 3.3 and 4) have been shown to be useful in testing our hypothesis.

The in vivo study described in chapter 5, was conducted to confirm our findings presented in chapters 3.1 and 3.2. The results from this in vivo study may be considered as an important step towards establishing causality between statin use and the risk of developing RA. Besides establishing the causality of the relationship between statin exposure and autoimmune disorders, experimental studies in mouse models have the advantage of allowing us to study the adverse effects of statins, such as statin-induced RA. Moreover, controlling for all factors related to statin exposure and the development of RA in human intervention studies is complicated because of practical and ethical reasons. Uncertainties in relation to extrapolation of data from animal studies to humans are, however, a main disadvantage of experimental animal studies. Causality can also be illustrated by RCTs ${ }^{157}$. However, RCTs are inefficient to detect rare adverse events or adverse events with a delayed outcome (e.g. autoimmune disorders). Furthermore, RCTs may be less generalisable due to, for instance, selection of patients without co-morbidities or co-medication, or patients' non-adherence to the drug therapy (e.g. statins) 44 . 


\section{Assessment of statin use}

For the observational studies described in chapters 3.1, 3.2 and 3.3 of this thesis, routinely recorded prescription data from GPs were used. An advantage of using a computerised database is that we do not have to rely on patients' recall of statin, ACE inhibitor, or ARB use, thereby eliminating recall bias. In addition, accurate data on the prescribed drugs over a long period of time are available. However, using computerised prescription data from the GP may introduce an overestimation of actual statin, ACE inhibitor, or ARB use.

In chapter 4 pharmacy-dispensing data were obtained from PHARMO-RLS which was linked to the Doetinchem Cohort Study. Practically, all inhabitants are registered with a single community pharmacy, independent of the prescriber. Pharmacy-dispensing data have similar advantages to prescription data from GPs. Pharmacy-dispending records; however, have the advantage over prescription records of including information about whether statins, ACE inhibitors, or ARBs were actually obtained from the pharmacist in addition to being only prescribed by the GP. In a study where the differences between prescribed and dispensed medications were examined, it was demonstrated that approximately $13 \%$ of the drugs prescribed in general practice are not dispensed. However, patients eligible for governments' reimbursement, as in the Netherlands, were more likely to have their prescriptions filled than patients without financial support 158 .

In a validation study of pharmacy records, it has been shown that such records are a reliable reflection of drug exposure as determined by a home inventory ${ }^{159}$. However, it is still not clear whether statins, ACE inhibitors, or ARBs were actually being taken according to physician's prescribed regimen. Furthermore, no information regarding the adherence to or discontinuation of therapy is available in electronic health record databases.

\section{Assessment of autoimmune disorders}

In the studies presented in chapters 2 and 3 of this thesis, autoimmune disorders were assessed by spontaneous case reports and routinely recorded morbidity data from GPs. Despite the fact that we have reports or electronic records from specific autoimmune disorders, we may have used a relatively sensitive, but non-specific, definition of lupus-like syndrome, PMR and RA. In chapters 2.1 and 2.2, the definition of lupus-like syndrome and PMR was based on ADR terms used in the ICSRs submitted by physicians, manufacturers and consumers. Regrettably, in these ADR reports no detailed description such as elevation of inflammatory markers (erythrocyte sedimentation rate (ESR) and CRP), or response to therapeutics and disease course, for diagnosing PMR or lupus-like syndrome were available. As a consequence, we were not able to recognise possible diagnostic misclassification 160,161. However, in at least one of the ADR reports, we were able to collect clinical data from the reporter 
which gave us the opportunity to describe the clinical course of a patient with statin-associated PMR/GCA in more detail (chapter 2.3). Furthermore, in a small number of ADR reports of lupus-like syndrome the presence of ANA was listed. Since the inception of the WHO Global ICSR database in the late 1960s 162 , lupus erythematosus (LE) cells could also have been listed in ADR reports of lupus-like syndrome. LE cell testing was once performed to diagnose lupus-like syndrome but has been replaced by testing for ANA and anti-double stranded DNA. To minimise possible misclassification of lupus-like syndrome, we conducted a sensitivity analysis where we only included ADR reports of lupus-like syndrome in which the presence of ANA and/or LE cells was listed. Another approach to minimise misclassification of lupus-like syndrome or PMR involved limiting the heterogeneity of the ADR reports by conducting a sensitivity analysis where we only included ADRs from physicians. All of the sensitivity analyses have shown the same results, indicating that possible misclassification of lupus-like syndrome or PMR did not have a substantial impact on the results.

In chapters 3.1, 3.2 and 3.3 we defined RA based on routinely recorded morbidity data from GPs. In both GP databases, we had sufficient numbers of cases of RA to perform a case-control study or cohort study. Furthermore, both databases comprised robust data on morbidities of the patients. Again, we could not assess a complete description of the patient, and therefore we may have used a relatively sensitive, but non-specific, definition of RA. To deal with potential misclassification of RA, the first-time diagnosis registered by a GP was verified by the use of disease-modifying anti-rheumatoid drugs (DMARDs), or corticosteroids, or referrals to a rheumatologist. In chapter 3.2, we defined RA based on a diagnostic algorithm as postulated by Thomas et al. 163. The proposed diagnostic algorithm classified patients as having RA when they received at least one prescription of DMARDs after their first-time diagnosis, resulting in a relatively accurate diagnosis of RA ${ }^{163}$. In chapters 3.1 and 3.3, we used a definition of patients with RA as those who received at least one prescription of DMARDs (as described in chapter 3.2), or at least two prescriptions of corticosteroids, or were referred to a rheumatologist. This increased the sample size of our cases of RA. By using stringent criteria for defining RA, we ultimately included a small number of patients with RA in our studies. Our restricted sample of patients with RA may have hampered the external validity of the studies. We evaluated the impact of changing definitions of RA in several sensitivity analyses. Our results remained unchanged when we changed the definition of RA. As mentioned previously, GP databases lack medical information from the rheumatologists. For instance, when DMARDs or corticosteroids were prescribed by the rheumatologist, the prescription of the patient may not always appear in these datasets, resulting in a possible underestimation of patients with RA. Otherwise, 
patients with other forms of arthritis, e.g., psoriatic arthritis may have appeared in these datasets. As a consequence, we may have introduced information bias that may produce an under- or over-estimation of the associations. Again, by assessing a complete medical history of the patient through data linkage, we may overcome the hurdle of possible misclassification of RA by using more specific markers for RA: RF and/or anti-CCP.

\section{Other methodological considerations}

Some additional methodological issues need to be considered when examining the associations between statin use and the risk of developing systemic autoimmune disorders. In our observational studies with RA, we could define the date of onset of RA by the first record of the GP for RA, but the date of onset of the RA symptoms is unknown ${ }^{164}$. Chan et al. reported that the median time between the onset of symptoms and the diagnosis of RA was approximately 36 weeks 165 . When there is a lag-time from the first symptoms and start of statin treatment before actual diagnosis of RA, protopathic bias may have occurred 166. In two previous population-based studies, it was considered that in the first year following initiation of statin treatment the development of RA may not be attributable to statins 67,69. To prevent this possible type of bias (protopathic bias) of defining the date of onset, we performed several sensitivity analyses where we shifted the date of the first-time diagnosis of RA to an earlier date (chapters 3.1, 3.2 and 3.3) and found similar, although somewhat more attenuated findings. In chapter 3.2 we performed an extra sensitivity analysis where we excluded the first year following the initiation of statin treatment from the follow-up time and found that the association between statin use and the risk of developing RA disappeared. Despite the results of our sensitivity analyses, we cannot exclude the possibility of protopathic bias. In addition, ascertainment or detection bias may have accounted to some degree for the increased risk of RA shortly after starting statin use. Patients who are treated with statins or patients who may experience myalgia or other muscle-related adverse effects due to statins; 167 tended to have more diagnostic testing or visits to their GP, or were more likely to be referred to a rheumatologist than the untreated patients, around the time of initiation of statin therapy; and may lead to an increased likelihood of being diagnosed with RA 68,168.

Furthermore, differences in diagnoses and prescribing regimens by general practice could have biased the results of our studies. In our two case-control studies (chapters 3.1 and 3.3), the average duration of registration with the GP is almost equal between patients with RA and controls, indicating that the patients in our studies had similar opportunities to have been prescribed a statin, or ACE inhibitor, or ARB. Because of the small number of patients with RA per GP, we could not control for general practice effects in our analyses. 
Another methodological consideration within observational studies is the phenomenon of 'confounding by indication' 169,170, which may occur when the prognosis of patients varies according to the exposure. By definition, characteristics differ inherently between those who were taking a given drug, and those who were not taking the drug, because they are taking the drug for a reason. Physicians have a reason to prescribe ('indication') drugs to patients, and tend to 'channel' certain drugs, or certain dosing regimens to specific patients. When these characteristics are related to the occurrence of the outcome, the association between exposure and outcome may be confounded. Importantly, cardiovascular risk factors (e.g. hyperlipidaemia) could have influenced the association between statin use and incident RA. In patients with RA, the systemic proinflammatory cytokines such as IL-1, IL-6 and tumor necrosis factor alpha (TNF-a) produced in synovial tissue of the joints, may promote the development of both traditional (e.g. hyperlipidaemia, hypertension, insulin resistance) and non-traditional (e.g. oxidative stress) systemic cardiovascular risk factors, thereby increasing the development of cardiovascular diseases (e.g. atherosclerosis) ${ }^{171}$. Several studies have demonstrated an unfavourable lipid profile in patients with RA 172,173 . Hyperlipidaemia may induce leukocyte activation and possibly complement activation, ${ }^{174-176}$ which may result in an earlier diagnosis of RA in patients prone to develop RA. When we conducted subgroup analyses in patients with and without these recorded cardiovascular risk factors, no differences in the risk of developing RA between these two groups were observed. Acknowledgement of the proinflammatory mechanisms in RA, atherosclerosis and hyperlipidaemia may lead to better recognition of cardiovascular co-morbidities in patients with RA 171 . However, it is still difficult to determine the onset of the cardiovascular co-morbidities. Since several studies have demonstrated an unfavourable lipid profile and hypertension in RA patients, or in patients who later develop RA 172,173, statin, ACE inhibitor, or ARB use could be a proxy for hyperlipidaemia or hypertension which may be responsible for the increased risk of developing RA in our studies. Taking the baseline characteristics from our studies into account, cardiovascular diseases and cardiovascular risk factors (e.g. hyperlipidaemia, hypertension) may have influenced our results. An approach to deal with confounding by indication involves restricting the study population to patients with a similar indication. Within cohort studies with a follow-up, this can either be achieved by comparing the incidence of events of interest between patients using comparative drugs, or to compare incidence rates within a single group of patients between the time when they used the drug and the time when they did not use the drug of interest 177,178. In order to limit confounding by indication in the case-control study, presented in chapter 3.3, we selected only patients who were prescribed anti-hypertensive drugs for the same indication ${ }^{179}$. By including these patients only, we have ensured that prognostic factors such as cardiovascular risk factors (e.g. hypertension, diabetes, hyperlipidaemia) were almost 
equally distributed between the RA patients and controls ${ }^{179}$. Regrettably, this strategy is not applicable in studies where the effects of statin use on the risk of developing autoimmune disorders will be evaluated. Since statins are the first choice agents in the treatment for patients with hyperlipidaemia, other lipid-lowering agents are less frequently prescribed. Due to the small sample size, it was not feasible to use other lipid-lowering medication users as an appropriate control group.

Another approach for limiting confounding by indication in observational studies is to match by characteristics (e.g. age, sex, cardiovascular disease, diabetes) that are related to both the risk of the future outcome (e.g. RA) 180,181 and the choice of drug therapy (statins) 182,183 , to ensure similar intervention groups with respect to important prognostic factors. We have used this technique in chapters 3 and 4 . However, in our cohort studies, the distribution of, e.g., the cardiovascular risk factors among the statin users and non-users remained unbalanced. Additionally, we conducted subgroup analyses in patients with the same indication (with or without cardiovascular diseases and cardiovascular related risk factors) as described in chapters 3.1, 3.2 and 4. Another method to minimise confounding by indication is to estimate propensity scores and analyse the data within homogenous levels of propensity scores. By using stratification or matching, for instance, by the propensity score a balance of covariates between the statin users and non-users may be achieved 179 . Finally, adjustment for confounding factors or inclusion of a single propensity score value (as a covariate) in the analyses by using multivariable regression analysis may minimise confounding by indication. The propensity score technique usually does not result in substantially different treatment effect estimates compared with the conventional regression methods 184,185 . The conventional regression and propensity score techniques, however, are similar in that they can only adjust for known and measured potential confounders ${ }^{186}$. We used multiple imputation methods (chapter 4) or a missing indicator method (missing data in the variables were treated as a separate category in the analysis) (chapter 3.2) to account for missing data. In order to deal with unmeasured confounding such as smoking status, we performed several simulation models with potential effects of smoking, thereby estimating the impact of smoking on the association between statin use and the risk of developing RA ${ }^{187}$ (chapter 3.1). Another approach that is used to reduce unmeasured confounding is by data linkage. An example is the Doetinchem cohort study, which is already linked to pharmacy-dispensing and hospital admission data from PHARMO-RLS; this may be extended with morbidity data from general practitioners, clinical and laboratory data, in- and outpatient medication use from hospitals, and samples and data from biobanks. One of the health care data linkage possibilities for the Doetinchem cohort study may be to link to the electronic patient file (in Dutch: Elektronisch Patiënten Dossier (EPD)) which would provide data covering all healthcare services in the Netherlands. Yet, ethical dilemmas play a major role in accessing the EPD system ${ }^{188}$, 
and data from this system would not be in an automated database format 188 . To deal with the ethical dilemmas of linking data, linkage of these individual databases could be professionally conducted by a trusted third party, as has been carried out in the Mondriaan project and LINH. The Mondriaan project and ZorgTTP (the trusted third party for LINH) facilitate linkage of several individual databases from health care providers and research projects of linked data research institutes in the Netherlands. By using a trusted third party, medical and research data can be separated from the name, address and postal code (encrypted). Through their settlement agreement, the Mondriaan project and LINH only receives medical and research data with certified anonymity, thereby guaranteeing patients' privacy 189,190.

Another possibility of linking data can involve encrypting patients' identification into a unique personal identification number, as in Denmark. In Denmark, each person is issued with a unique personal identification number upon birth or immigration. This unique personal identification number encodes, among other information, date of birth and sex, and by that means, information on a given subject can be linked to multiple data sources 191,192 .

\section{PRACTICAL AND CLINICAL IMPLICATIONS}

Based on the findings reported in this thesis and the literature we would like to make several recommendations for clinical practice.

\section{Reporting adverse drug reactions}

Despite the pro-active and routine approaches used in pharmacovigilance, underreporting of the ADRs is extremely high and expected to be approximately $90 \%$ 50. Additionally, selective underreporting may have occurred since the reporter may not always be alert on the ADR, and therefore; the assessment of an association between drug intake and an adverse event remains a challenge. However, collection of ADRs is warranted for signal detection (hypothesis strengthening) and hypothesis generation. In this thesis, we have shown that two decades after approval for marketing, reporting of lupus-like syndrome and PMR occurred more often with statins than with other drugs.

We urge physicians, pharmacists and other healthcare providers, and above all, patients to report potential side effects to pharmacovigilance centres. Patients have been invited to report ADRs directly in the US, Canada and New Zealand 193. Since the European Parliament legislative resolution on pharmacovigilance of medicinal products for human use (Regulation [EC] No 726/2004) which was launched in July 2012 194, patients from all European member states are allowed to report concerns regarding drugs, directly to the competent authorities by web-based structured forms 195,196. At the Dutch pharmacovigilance centre, Lareb, every ADR from patients is 
stored in a database and compared with previous national and international ADRs. All potential ADR signals are sent to the Dutch regulatory authority and European Medicines Agency 197.

The information reported by patients and healthcare professionals is generally comparable, although patients' reports on ADRs tend to provide more information on the impact of the reported ADRs whereas reports from healthcare professionals focus on diagnosis related information. Both types of information are valuable to pharmacovigilance centres 198,199. In studies using databases containing spontaneously reported ADRs, we have shown that excluding ADRs submitted by patients did not change our findings (chapters 2.1 and 2.2), indicating that ADRs submitted by patients are useful for signal detection and hypothesis generation.

\section{Inform physicians and pharmacists of statin-associated autoimmune disorders}

In general, statins are considered to be safe although the market withdrawal of cerivastatin has demonstrated that some serious adverse effects are not always detected in clinical trials. The most commonly reported side effects of statins are marked elevation of liver enzymes and muscular abnormalities 200. Our findings raise awareness about the possibility of developing lupus-like syndrome, PMR or RA in patients treated with statins. For instance, when a patient on statin therapy presents with myalgia of hip and shoulder region in combination with morning stiffness, one should consider the possibility of PMR. Physicians and pharmacists should be informed about these possible statin-associated autoimmune disorders.

\section{Prescribing statins in low cardiovascular risk patients}

According to several guidelines, patients with a high risk of cardiovascular disease should be treated with statins 182,183,201-205. For instance, according to the European guideline on cardiovascular disease prevention in clinical practice, patients with a 10 -year risk of cardiovascular disease death of $\geq 5 \%$ (based on the Systematic Coronary Risk Evaluation Project (SCORE) chart) should be on statin treatment 182. In patients with a low cardiovascular risk (SCORE level of $\leq 5 \%$ ), statin therapy may be considered when lifestyle modification such as smoking cessation, regular physical activity and/or dietary changes is not sufficient 182 . However, the findings in this thesis shed new light on the benefit-risk ratio of statins. We found that statin use was associated with an increased occurrence or risk of developing lupus-like syndrome, PMR or RA. Our findings may slightly alter the 'risk' component of the benefit-risk ratio. However, others have not corroborated these findings 66-70, and therefore; more definitive studies in healthy (low-risk) subjects are needed. Until this question is resolved, one should be conservative in prescribing statins to individuals with a low cardiovascular risk. 


\section{Update drug labels}

In daily clinical practice, drug labels can play a pivotal role in signalling the risk of rare autoimmune disorders. Drug labels inform healthcare professionals on the safe and effective use of a specific drug. According to the guideline for the summary of product characteristics 206, a list of all adverse reactions with their respective frequency and the source of the safety database (e.g. from clinical trials, post-authorisation safety studies or spontaneous reporting) should be enclosed. So far, data on statin-associated immune-mediated disorders has only been based on case reports. In this thesis, we have conducted several studies with different designs (e.g. observational, experimental, case history) and data sources (e.g. spontaneous reporting, electronic health records, laboratory and in vivo), and all of them showed an increased occurrence or risk of developing autoimmune disorders when individuals were treated with statins. Based on this information, we suggest that it is time to update the information on the label.

\section{Develop a validated risk tool to predict statin-associated autoimmune disorders}

Despite the increase in statin use in last two decades, only a small number of patients who were treated with these drugs over a prolonged period of time developed a systemic statin-induced syndrome ${ }^{8}$. While the absolute number of patients is low, statin-associated autoimmune disorders may potentially impose a heavy burden on public health, since these disorders can be chronic, with long-term morbidity and may account for high healthcare costs, loss of quality of life and loss of productivity. Therefore, careful risk assessment should be considered. Patients who are susceptible to autoimmune disorders may have a high risk of developing these disorders when treated with a statin. A validated risk prediction tool, such as developing biomarkers may therefore be useful to predict statin-associated autoimmune disorders. Recently, autoantibodies, that recognise 3-hydroxy-3-methylglutaryl-coenzyme A reductase (HMGCR) have been identified in patients with statin-associated autoimmune myopathy 207, whereas in a large population of statin exposed patients without myopathy no anti-HMGCR antibodies were found 208 . Although to date, no validated biomarkers are available for predicting statin-associated autoimmune disorders, we propose that such risk prediction tools could potentially help healthcare professionals to categorise patients based on risk profiles. 


\section{RECOMMENDATIONS FOR FUTURE RESEARCH}

After studying the effects on the development of autoimmune disorders of the commonly prescribed drugs such as statins, ACE inhibitors and ARBs, some research questions remained unanswered and/or new questions have been triggered.

Although both ACE inhibitors and ARBs can be considered to be safe and well tolerated with regard to RA risk, even after prolonged use, further research using a larger sample size and other electronic health care databases (e.g. health administrative databases), and taking other autoimmune disorders related events into account are needed to confirm our findings as presented in chapter 3.3.

Since we are the first to observe an association between statin use and an increased risk of autoimmune disorders, our findings should be replicated by additional observational studies, including a large post-approval study following more than 40000 patients for at least 10 years after initiation of statin treatment.

We have detected potential signals for statin-associated lupus-like syndrome and PMR, and these findings should be further confirmed and the risk should be quantified by large retrospective cohort studies.

In the future, more databases such as data from registries and biobanks should be linked and extended with other types of data, to investigate the association between statin use and autoimmunity/immune disorders in detail, and to address the methodological challenges, as outlined in this chapter.

To understand the findings presented in this thesis, the mechanisms that have been proposed in this chapter should be further unravelled in experimental and clinical studies.

As statin, ACE inhibitor or ARB use might be considered as a proxy for hyperlipidaemia or hypertension, more clinical and experimental studies should be conducted to elucidate whether, hyperlipidaemia or hypertension, or the immunomodulatory properties of statins, ACE inhibitors or ARBs is primarily responsible for the increased risk of developing autoimmune disorders.

To address our hypothesis that statins may promote a pre-existing autoimmune-prone condition to develop into a clinically manifest disease. Patient data, e.g., genotyped and structurally screened risk factors, co-morbidities and co-medication, should be available and stored in a large database, as has been carried out by researchers in the Rainbow-projects of the Dutch Biobanking and Biomolecular Resource Research Infrastructure (BBMRI-NL) 209.

When cohort studies are not feasible for practical and economic reasons, the use of a nested case-control study is a reasonable and valid alternative for studying associations between statin use and autoimmune disorders since the person time of every individual is also taken into account. A nested case-control study is a 
special type of case-control study in which 'cases' of an autoimmune disorder that arise during the follow-up period are selected from the same cohort (population of people) as the controls (who are at risk for developing an autoimmune disorder at the case's date of first diagnosis) to whom they are compared. This study is thus said to be 'nested' within the cohort study 210 .

When cohort studies are feasible, we recommend the use of time-dependent exposure classification (chapter 3.2), whenever applicable, in order to determine the absolute risk estimates and minimise immortal time bias. Immortal time bias refers to a period of cohort follow-up during which, by design, the event (e.g. RA or death) cannot occur 211.

When taking a time-dependent cohort methodology into consideration, we strongly encourage using hazard pattern analyses when the relative risks are not constant over time and to detect the period when drug users are sensitive to developing drug-induced autoimmune disorders.

More studies focussing on the development of autoimmune disorders in the first year after initiation of statins, in particular in a selective group of patients prone to autoimmune disorders are needed to disentangle whether the genetic susceptibility to an autoimmune disorder, or ascertainment bias, or detection bias is mainly responsible for the increased risk of developing autoimmune disorders

Data mining studies that combine clinical, laboratory, experimental and pharmacoepidemiology methods have shown promising results when investigating the relationships between the cardiovascular drugs and the risk of developing autoimmune disorders. Therefore, multidisciplinary research should be encouraged.

\section{CONCLUSION}

In conclusion, this thesis shows that use of statins may be associated with an increased risk of developing autoimmune disorders. Current evidence shows that statins are associated with an increased occurrence or risk of developing lupus-like syndrome, PMR or RA. The risk of developing RA is apparent in the first year of statin use. Moreover, we have demonstrated causality between statin use and the risk of developing autoimmune disorders. On the other hand, in healthy subjects statin use was not associated with a loss of self-tolerance. In contrast to statins, we demonstrated that both ACE inhibitors and ARBs can be considered as safe and well tolerated with regard to $R A$, even after prolonged use.

The findings of this thesis add pieces to the puzzle of the cardiovascular drug-associated autoimmune disorder hypothesis, however, a number of issues remain unsolved.

Firstly, statins may not cause autoimmunity by themselves but may promote a preexisting autoimmune-prone condition to progress towards clinically manifest diseases. 
Linkage of biobank data to population health data and pharmacy-dispensing records would be helpful in this respect. Research is needed to study this hypothesis in detail. Secondly, one can argue whether we have used the most relevant outcome definitions and confounders. By assessing a complete medical history of the patient through linkage of datasets, it may be possible to minimise misclassification of lupus-like syndrome, PMR or RA, or protopathic bias, or to improve the quality and quantity of confounding factors.

Thirdly, statin, ACE inhibitor or ARB use could serve as a proxy for hyperlipidaemia or hypertension. More research should be conducted to elucidate whether hyperlipidaemia or hypertension, or the immunomodulatory properties of statins, ACE inhibitors or ARBs is primarily responsible for the increased risk of developing autoimmune disorders.

Since our findings have not been corroborated by others, multidisciplinary research designed to confirm, explain in detail, and establish causality of the association between the use of cardiovascular drugs, particularly statins, ACE inhibitors and ARBs, and the incidence of autoimmune disorders is of the utmost importance. 


\section{REFERENCES}

1. Molina V, Shoenfeld Y. Infection, vaccines and other environmental triggers of autoimmunity. Autoimmunity 2005; 38(3): 235-45.

2. Marrack P, Kappler J, Kotzin BL. Autoimmune disease: why and where it occurs. Nat Med 2001; 7(8): 899-905

3. Klareskog L, Malmström V, Lundberg K, Padyukov L, Alfredsson L. Smoking, citrullination and genetic variability in the immunopathogenesis of rheumatoid arthritis. Semin Immunol 2011; 23(2): 92-8.

4. Singh N, Singh U, Singh B, Price R, Nagarkatti M, Nagarkatti P. Activation of aryl hydrocarbon receptor (AhR) leads to reciprocal epigenetic regulation of FoxP3 and IL-17 expression and amelioration of experimental colitis. PLoS ONE 2011; 6(8): e23522.

5. Todd J. D'oh! genes and environment cause Crohn's disease. Cell 2010; 141(7): 1114-6.

6. WHO Collaborating Centre for Immunotoxicology and Allergic Hypersensitivity. Principles and methods for assessing autoimmunity associated with exposure to chemicals: Environmental Health Criteria. Bilthoven, The Netherlands: World Health Organisation, 2006.

7. Wiik A. Drug-induced vasculitis. Curr Opin Rheumatol 2008; 20(1): 35-9.

8. Vasoo S. Drug-induced lupus: an update. Lupus 2006; 15(11): 757-61.

9. Vedove CD, Del Giglio M, Schena D, Girolomoni G. Drug-induced lupus erythematosus. Arch Dermatol Res 2009; 301(1): 99-105.

10. Olsen N. Drug-induced autoimmunity. Best Pract Res Clin Rheumatol 2004; 18(5): 677-88.

11. Mor A, Pillinger M, Wortmann R, Mitnick H. Drug-induced arthritic and connective tissue disorders. Semin Arthritis Rheum 2008; 38(3): 249-64.

12. Mongey A, Hess E. Drug insight: autoimmune effects of medications-what's new? Nat Clin Pract Rheumatol 2008; 4(3): 136-44.

13. Borchers AT, Keen CL, Gershwin ME. Drug-induced lupus. Ann N Y Acad Sci 2007; 1108: 166-82.

14. Choi HK, Merkel PA, Walker AM, Niles JL. Drug-associated antineutrophil cytoplasmic antibody-positive vasculitis: prevalence among patients with high titers of antimyeloperoxidase antibodies. Arthritis rheumatism 2000; 43(2): 405-13.

15. Dolman KM, Gans RO, Vervaat TJ, et al. Vasculitis and antineutrophil cytoplasmic autoantibodies associated with propylthiouracil therapy. Lancet 1993; 342(8872): 651-2.

16. Hess E. Drug-related lupus. N Engl J Med 1988; 318(22): 1460-2.

17. Marzano AV, Vezzoli P, Crosti C. Drug-induced lupus: an update on its dermatologic aspects. Lupus 2009; 18(11): 935-40.

18. Rubin RL. Drug-induced lupus. Toxicology 2005; 209(2): 135-47.

19. Uetrecht J. Current trends in drug-induced autoimmunity. Autoimmun Rev 2005; 4(5): 309-14.

20. Law MR, Wald NJ, Rudnicka AR. Quantifying effect of statins on low density lipoprotein cholesterol, ischaemic heart disease, and stroke: systematic review and meta-analysis. BMJ 2003; 326(7404): 1423.

21. PROGRESS Collaborative Group. Randomised trial of a perindopril-based blood-pressure-lowering regimen among 6,105 individuals with previous stroke or transient ischaemic attack. Lancet 2001; 358(9287): 1033-41.

22. MacMahon S, Peto R, Cutler J, et al. Blood pressure, stroke, and coronary heart disease. Part 1, Prolonged differences in blood pressure: prospective observational studies corrected for the regression dilution bias. Lancet 1990; 335(8692): 765-74.

23. Collins R, Peto R, MacMahon S, et al. Blood pressure, stroke, and coronary heart disease. Part 2, Short-term reductions in blood pressure: overview of randomised drug trials in their epidemiological context. Lancet 1990; 335(8693): 827-38.

24. Antiplatelet Trialists' Collaboration. Collaborative overview of randomised trials of antiplatelet therapy--I: Prevention of death, myocardial infarction, and stroke by prolonged antiplatelet therapy in various categories of patients. BMJ 1994; 308(6921): 81-106.

25. Antithrombotic Trialists' Collaboration. Collaborative meta-analysis of randomised trials of antiplatelet therapy for prevention of death, myocardial infarction, and stroke in high risk patients. BMJ 2002; 324(7329): 71-86. 
26. Platten M, Youssef S, Hur EM, et al. Blocking angiotensin-converting enzyme induces potent regulatory T cells and modulates TH1- and TH17-mediated autoimmunity. Proc Natl Acad Sci U S A 2009; 106(35): 14948-53.

27. Sukumaran V, Watanabe K, Veeraveedu PT, et al. Olmesartan, an AT1 antagonist, attenuates oxidative stress, endoplasmic reticulum stress and cardiac inflammatory mediators in rats with heart failure induced by experimental autoimmune myocarditis. Int J Biol Sci 2011; 7(2): 154-67.

28. Ruiz-Ortega M, Bustos C, Hernández-Presa MA, Lorenzo O, Plaza JJ, Egido J. Angiotensin Il participates in mononuclear cell recruitment in experimental immune complex nephritis through nuclear factor-kappa B activation and monocyte chemoattractant protein-1 synthesis. J Immunol 1998; 161(1): 430-9.

29. Schindler R, Dinarello CA, Koch KM. Angiotensin-converting-enzyme inhibitors suppress synthesis of tumour necrosis factor and interleukin 1 by human peripheral blood mononuclear cells. Cytokine 1995; 7(6): 526-33.

30. Fukuzawa M, Satoh J, Sagara M, et al. Angiotensin converting enzyme inhibitors suppress production of tumor necrosis factor-alpha in vitro and in vivo. Immunopharmacology 1997; 36(1): 49-55.

31. Constantinescu CS, Goodman DB, Ventura ES. Captopril and lisinopril suppress production of interleukin-12 by human peripheral blood mononuclear cells. Immunol Lett 1998; 62(1): 25-31.

32. Martin MF, Surrall KE, McKenna F, Dixon JS, Bird HA, Wright V. Captopril: a new treatment for rheumatoid arthritis? Lancet 1984; 1(8390): 1325-8.

33. Perry ME, Chee MM, Ferrell WR, Lockhart JC, Sturrock RD. Angiotensin receptor blockers reduce erythrocyte sedimentation rate levels in patients with rheumatoid arthritis. Ann Rheum Dis 2008; 67(11): 1646-7.

34. Ridker PM, Danielson E, Fonseca FA, et al. Reduction in C-reactive protein and LDL cholesterol and cardiovascular event rates after initiation of rosuvastatin: a prospective study of the JUPITER trial. Lancet 2009; 373(9670): 1175-82.

35. Weitz Schmidt G, Welzenbach K, Brinkmann V, et al. Statins selectively inhibit leukocyte function antigen-1 by binding to a novel regulatory integrin site. Nat Med 2001; 7(6): 687-92.

36. Youssef S, Stüve O, Patarroyo JC, et al. The HMG-CoA reductase inhibitor, atorvastatin, promotes a Th2 bias and reverses paralysis in central nervous system autoimmune disease. Nature 2002; 420(6911): 78-84.

37. Vollmer T, Key L, Durkalski V, et al. Oral simvastatin treatment in relapsing-remitting multiple sclerosis. Lancet 2004; 363(9421): 1607-8.

38. Neuhaus O, Stüve O, Zamvil SS, Hartung HP. Are statins a treatment option for multiple sclerosis? Lancet Neurol 2004; 3(6): 369-71.

39. McCarey DW, Mclnnes IB, Madhok R, et al. Trial of Atorvastatin in Rheumatoid Arthritis (TARA): double-blind, randomised placebo-controlled trial. Lancet 2004; 363(9426): 2015-21

40. Leung BP, Sattar N, Crilly A, et al. A novel anti-inflammatory role for simvastatin in inflammatory arthritis. J Immunol 2003; 170(3): 1524-30

41. Kwak BR, Mulhaupt F, Mach F. Atherosclerosis: anti-inflammatory and immunomodulatory activities of statins. Autoimmun Rev 2003; 2(6): 332-8.

42. Arnaud C, Braunersreuther V, Mach F. Toward immunomodulatory and anti-inflammatory properties of statins. Trends Cardiovasc Med 2005; 15(6): 202-6.

43. López-Pedrera C, Aguirre MÃ, Barbarroja N, Cuadrado MJ. Accelerated atherosclerosis in systemic lupus erythematosus: role of proinflammatory cytokines and therapeutic approaches. J Biomed Biotechnol 2010; 2010: pii:607084.

44. Stricker BH, Psaty BM. Detection, verification, and quantification of adverse drug reactions. BMJ 2004; 329(7456): 44-7.

45. Poluzzi E, Raschi E, Koci A, et al. Antipsychotics and torsadogenic risk: signals emerging from the US FDA Adverse Event Reporting System database. Drug Saf 2013; 36(6): 467-79.

46. Hoffman KB, Kraus C, Dimbil M, Golomb BA. A survey of the FDA's AERS database regarding muscle and tendon adverse events linked to the statin drug class. PLoS One 2012; 7(8): e42866.

47. Eichler HG, Pignatti F, Flamion B, Leufkens $H$, Breckenridge A. Balancing early market access to new drugs with the need for benefit/risk data: a mounting dilemma. Nat Rev Drug Discov 2008; 7(10): 818-26. 
48. International Conference on Harmonisation of Technical Requirements for Registration of Pharmaceuticals for Human Use. ICH Harmonised Tripartite Guideline Pharmacovigilance Planning E2E. November 2004. Available from: http://www.ich.org/fileadmin/Public_Web_Site/ICH_Products/Guidelines/Efficacy/ E2E/Step4/E2E_Guideline.pdf [accessed 23 December 2013].

49. International Conference on Harmonisation of Technical Requirements for Registration of Pharmaceuticals for Human Use. ICH Harmonised Tripartite Guideline Immunotoxicity Studies for Human Pharmaceuticals S8. September 2005; Available from: http://www.ich.org/fileadmin/Public_Web_Site/lCH_ Products/Guidelines/Safety/S8/Step4/S8_Guideline.pdf [accessed 23 December 2013].

50. Hazell L, Shakir SA. Under-reporting of adverse drug reactions: a systematic review. Drug Saf 2006; 29(5): 385-96.

51. Noël B. Statins and lupus erythematosus. Rheumatology 2004; 43(3): 397-8.

52. Noël B, Panizzon R. Lupus-like syndrome associated with statin therapy. Dermatology 2004; 208(3): 276-7.

53. Noël B. Risks and benefits of statins in lupus erythematosus. Arch Intern Med 2004; 164(1): 107-8

54. Krukemyer JJ, Talbert RL. Lovastatin: a new cholesterol-lowering agent. Pharmacotherapy 1987; 7(6): 198-210.

55. Haroon M, Devlin J. A case of ANCA-associated systemic vasculitis induced by atorvastatin. Clin Rheumatol 2008; 27 Suppl 2: S75-7.

56. Sen D, Rosenstein E, Kramer N. ANCA-positive vasculitis associated with simvastatin/ezetimibe: expanding the spectrum of statin-induced autoimmunity? Int J Rheum Dis 2010; 13(3): e29-31.

57. Goëb V, Guillemant N, Vittecoq O, Le Loët X. Cerivastatin-induced polymyalgia rheumatica-like illness. Clin Rheumatol 2004; 23(2): 179.

58. Kay J, Finn D, Stone J. Case records of the Massachusetts General Hospital. Case 4-2006. A 79-year-old woman with myalgias, fatigue, and shortness of breath. N Engl J Med 2006; 354(6): 623-30.

59. Rudski L, Rabinovitch MA, Danoff D. Systemic immune reactions to HMG-CoA reductase inhibitors. Report of 4 cases and review of the literature. Medicine 1998; 77(6): 378-83.

60. Noël B. Lupus erythematosus and other autoimmune diseases related to statin therapy: a systematic review. J Eur Acad Dermatol and Venereol 2007; 21(1): 17-24.

61. Golomb BA, Evans MA. Statin adverse effects : a review of the literature and evidence for a mitochondrial mechanism. Am J Cardiovasc Drugs 2008; 8(6): 373-418.

62. Moulis G, Béné J, Sommet A, Sailler L, Lapeyre-Mestre M, Montastruc JL, French Association of PharmacoVigilance Centres. Statin-induced lupus: a case/non-case study in a nationwide pharmacovigilance database. Lupus 2012; 21(8): 885-9.

63. Lewallen S, Courtright P. Epidemiology in practice: case-control studies. Community Eye Health 1998; 11(28): 57-8.

64. Myasoedova E, Crowson CS, Kremers HM, Therneau TM, Gabriel SE. Is the incidence of rheumatoid arthritis rising?: results from Olmsted County, Minnesota, 1955-2007. Arthritis Rheum 2010; 62(6): 1576-82.

65. Alamanos Y, Drosos AA. Epidemiology of adult rheumatoid arthritis. Autoimmun Rev 2005; 4(3): 130-6.

66. Jick SS, Choi H, Li L, Mclnnes IB, Sattar N. Hyperlipidaemia, statin use and the risk of developing rheumatoid arthritis. Ann Rheum Dis 2009; 68(4): 546-51.

67. Chodick G, Amital H, Shalem Y, Kokia E, Heymann AD, Porath A, Shalev V. Persistence with statins and onset of rheumatoid arthritis: a population-based cohort study. PLoS Med 2010; 7(9): e1000336.

68. Hippisley-Cox J, Coupland C. Unintended effects of statins in men and women in England and Wales: population based cohort study using the QResearch database. BMJ 2010; 340: c2197.

69. Smeeth L, Douglas I, Hall AJ, Hubbard R, Evans S. Effect of statins on a wide range of health outcomes: a cohort study validated by comparison with randomized trials. Br J Clin Pharmacol 2009; 67(1): 99-109.

70. Schmidt T, Battafarano DF, Mortensen EM, Frei CR, Mansi I. Frequency of development of connective tissue disease in statin-users versus nonusers. Am J Cardiol 2013; 112(6): 883-8.

71. Rosenbaum PR, Rubin DB. The central role of the propensity score in observational studies for causal effects. Biometrika 1983; 70: 41-55.

72. Belitser SV, Martens EP, Pestman WR, Groenwold RH, de Boer A, Klungel OH. Measuring balance and model selection in propensity score methods. Pharmacoepidemiol Drug Saf 2011; 20(11): 1115-29. 
73. Patri P, Nigro A, Rebora A. Lupus erythematosus-like eruption from captopril. Acta Derm Venereol 1985; 65(5): 447-8

74. Sieber C, Grimm E, Follath F. Captopril and systemic lupus erythematosus syndrome. BMJ 1990; 301(6753): 669.

75. Bertin P, Kamdem J, Bonnet C, Arnaud M, Treves R. Captopril-induced lupus. Clin Exp Rheumatol 1993; 11(6): 695.

76. Pelayo M, Vargas V, Gonzales A, Vallano A, Esteban R, Guardia J. Drug-induced lupus-like reaction and captopril. Ann Pharmacother 1993; 27(12): 1541-2.

77. Fernández-Diaz ML, Herranz P, Suárez-Marrero MC, Borbujo J, Manzano R, Casado M. Subacute cutaneous lupus erythematosus associated with cilazapril. Lancet 1995; 345(8946): 398.

78. Ratliff NB 3rd. Captopril induced lupus. J Rheumatol 2002; 29(8): 1807-8.

79. Disdier P, Harle JR, Verrot D, Jouglard J, Weiller PJ. Adult Schönlein-Henoch purpura after lisinopril. Lancet 1992; 340(8825): 985.

80. Moots RJ, Keeling PJ, Morgan SH. Adult Schönlein-Henoch purpura after enalapril. Lancet 1992; 340(8814): 304-5.

81. Gonçalves R, Cortez Pinto H, Serejo F, Ramalho F. Adult Schönlein-Henoch purpura after enalapril. J Intern Med 1998; 244(4): 356-7.

82. Gupta S, Gandhi NM, Ferguson J. Cutaneous vasculitis secondary to ramipril. J Drugs Dermatol 2004; 3(1): 81-2.

83. Pinto GM, Lamarao P, Vale T. Captopril-induced pemphigus vegetans with Charcot-Leyden crystals. J Am Acad Dermatol 1992; 27(2 Pt 2): 281-4.

84. Kaplan RP, Potter TS, Fox JN. Drug-induced pemphigus related to angiotensin-converting enzyme inhibitors. J Am Acad Dermatol 1992; 26(2 Pt 2): 364-6

85. Ruocco V, Satriano RA, Guerrera V. "Two-step” pemphigus induction by ACE-inhibitors. Int J Dermatol 1992; 31(1): 33-6.

86. Kuechle MK, Hutton KP, Muller SA. Angiotensin-converting enzyme inhibitor-induced pemphigus: three case reports and literature review. Mayo Clin Proc 1994; 69(12): 1166-71.

87. Bastiaens MT, Zwan NV, Verschueren GL, Stoof TJ, Nieboer C. Three cases of pemphigus vegetans: induction by enalapril--association with internal malignancy. Int J Dermatol 1994; 33(3): 168-71.

88. Butt A, Burge SM. Pemphigus vulgaris induced by captopril. Br J Dermatol 1995; 132(2): 315-6.

89. Vignes S, Paul C, Flageul B, Dubertret L. Ramipril-induced superficial pemphigus. Br J Dermatol 1996; 135(4): 657-8.

90. Buzón E, Perez-Bernal AM, De la Pe a F, Ríos JJ, Camacho F. Pemphigus foliaceus associated with cilazapril. Acta Derm Venereol 1998; 78(3): 227.

91. Ong CS, Cook N, Lee S. Drug-related pemphigus and angiotensin converting enzyme inhibitors. Australas J Dermatol 2000; 41(4): 242-6.

92. Parodi A, Cozzani E, Milesi G, Drosera M, Rebora A. Fosinopril as a possible pemphigus-inducing drug. Dermatology 2002; 204(2): 139-41.

93. Patterson CR, Davies MG. Pemphigus foliaceus: an adverse reaction to lisinopril. J Dermatolog Treat 2004; 15(1): 60-2.

94. Bae YI, Yun SJ, Lee SC, Park GT, Lee JB. Pemphigus foliaceus induced by an angiotensin II receptor blocker. Clin Exp Dermatol 2008; 33(6): 721-3.

95. Sagawa K, Nagatani K, Komagata Y, Yamamoto K. Angiotensin receptor blockers suppress antigen-specific T cell responses and ameliorate collagen-induced arthritis in mice. Arthritis Rheum 2005; 52(6): 1920-8.

96. Dalbeth N, Edwards J, Fairchild S, Callan M, Hall FC. The non-thiol angiotensin-converting enzyme inhibitor quinapril suppresses inflammatory arthritis. Rheumatology (Oxford) 2005; 44(1): 24-31.

97. Sakuta T, Morita Y, Satoh M, Fox DA, Kashihara N. Involvement of the renin-angiotensin system in the development of vascular damage in a rat model of arthritis: effect of angiotensin receptor blockers. Arthritis Rheum 2010; 62(5): 1319-28.

98. Price A, Lockhart JC, Ferrell WR, Gsell W, McLean S, Sturrock RD. Angiotensin II type 1 receptor as a novel therapeutic target in rheumatoid arthritis: in vivo analyses in rodent models of arthritis and ex vivo analyses in human inflammatory synovitis. Arthritis Rheum 2007; 56(2): 441-7. 
99. Bird HA, Le Gallez P, Dixon JS, et al. A clinical and biochemical assessment of a nonthiol ACE inhibitor (pentopril; CGS-13945) in active rheumatoid arthritis. J Rheumatol 1990; 17(5): 603-8.

100. Tikiz C, Utuk O, Pirildar T, et al. Effects of Angiotensin-converting enzyme inhibition and statin treatment on inflammatory markers and endothelial functions in patients with longterm rheumatoid arthritis. J Rheumatol 2005; 32(11): 2095-101.

101. Cohen Tervaert JW. Hypertension: an autoimmune disease? Hypertens Res 2011; 34(4): 443-4.

102. Dessein PH, Norton GR. Should patients with RA be aggressively monitored for hypertension? Nat Clin Pract Rheumatol 2008; 4(1): 18-9.

103. Gurevich V, Shovman O, Slutzky L, Meroni P, Shoenfeld Y. Statins and autoimmune diseases. Autoimmun Rev 2005; 4(3): 123-9.

104. Peters SAE, Palmer MK, Grobbee DE, Crouse JR, O'Leary DH, Raichlen JS, Bots ML. C-reactive protein lowering with rosuvastatin in the METEOR study. J Intern Med 2010; 268(2): 155-61.

105. Yoon SS, Dillon CF, Carroll M, Illoh K, Ostchega Y. Effects of statins on serum inflammatory markers: the U.S. National Health and Nutrition Examination Survey 1999-2004. J Atheroscler Thromb 2010; 17(11): 1176-82.

106. Lyngdoh T, Vollenweider P, Waeber G, Marques-Vidal P. Association of statins with inflammatory cytokines: a population-based Colaus study. Atherosclerosis 2011; 219(1): 253-8.

107. Cherfan P, Tompa A, Wikby A, Löfgren S, Jonasson L. Effects of simvastatin on human $T$ cells in vivo. Atherosclerosis 2007; 193(1): 186-92.

108. Albert MA, Danielson E, Rifai N, Ridker PM, PRINCE Investigators. Effect of statin therapy on C-reactive protein levels: the pravastatin inflammation/CRP evaluation (PRINCE): a randomized trial and cohort study. JAMA 2001; 286(1): 64-70.

109. Ridker PM, Danielson E, Fonseca FA, et al. Rosuvastatin to prevent vascular events in men and women with elevated C-reactive protein. N Engl J Med 2008; 359(21): 2195-207.

110. Arora M, Chen L, Paglia M, et al. Simvastatin promotes Th2-type responses through the induction of the chitinase family member Ym1 in dendritic cells. Proc Natl Acad Sci U S A 2006; 103(20): 7777-82.

111. Fosso CK, Miller MJ, Solomon WR, Baker JR, Jr. Adverse effects of 3-hydroxy-3-methylglutaryl coenzyme A reductase inhibitors associated with elevated serum IgE and eosinophilia. J Allergy Clin Immunol 1995; 95(5 Pt 1): 1053-5.

112. Mulder DJ, Van Haelst PL, Wobbes MH, et al. The effect of aggressive versus conventional lipid-lowering therapy on markers of inflammatory and oxidative stress. Cardiovasc Drugs Ther 2007; 21(2): 91-7.

113. Fehr T, Kahlert C, Fierz W, et al. Statin-induced immunomodulatory effects on human $\mathrm{T}$ cells in vivo. Atherosclerosis 2004; 175(1): 83-90.

114. Walter RB, Fuchs D, Weiss G, Walter TR, Reinhart WH. HMG-CoA reductase inhibitors are associated with decreased serum neopterin levels in stable coronary artery disease. Clin Chem Lab Med 2003; 41(10): 1314-9

115. Van Haelst PL, Van Doormaal JJ, May JF, Gans RO, Crijns HJ, Tervaert JW. Secondary prevention with fluvastatin decreases levels of adhesion molecules, neopterin and C-reactive protein. Eur J Intern Med 2001; 12(6): 503-9.

116. Bevaart L, Vervoordeldonk MJ, Tak PP. Collagen-induced arthritis in mice. Methods Mol Biol 2010; 602: 181-92.

117. Ho PP, Higgins JP, Kidd BA, et al. Tolerizing DNA vaccines for autoimmune arthritis. Autoimmunity 2006; 39(8): 675-82.

118. Yamagata T, Kinoshita K, Nozaki Y, Sugiyama M, Ikoma S, Funauchi M. Effects of pravastatin in murine collagen-induced arthritis. Rheumatol Int 2007; 27(7): 631-9.

119. Palmer G, Chobaz V, Talabot-Ayer D, Taylor S, So A, Gabay C, Busso N. Assessment of the efficacy of different statins in murine collagen-induced arthritis. Arthritis Rheum 2004; 50(12): 4051-9.

120. Kagami $\mathrm{S}$, Owada $\mathrm{T}$, Kanari $\mathrm{H}$, et al. Protein geranylgeranylation regulates the balance between Th17 cells and Foxp3 regulatory T cells. Int Immunol 2009; 21(6): 679-89.

121. Esensten $\mathrm{JH}$, Wofsy $\mathrm{D}$, Bluestone JA. Regulatory T cells as therapeutic targets in rheumatoid arthritis. Nat Rev Rheumatol 2009; 5(10): 560-5.

122. Tang TT, Song Y, Ding YJ, et al. Atorvastatin up-regulates regulatory T-cell and improves clinical disease activity in patients with rheumatoid arthritis. J Lipid Res 2011; 52(5): 1023-32. 
123. Zhou X, Bailey-Bucktrout SL, Jeker LT, et al. Instability of the transcription factor Foxp3 leads to the generation of pathogenic memory T cells in vivo. Nat Immunol 2009; 10(9): 1000-7.

124. Komatsu N, Mariotti-Ferrandiz ME, Wang Y, Malissen B, Waldmann H, Hori S. Heterogeneity of natural Foxp3+ T cells: a committed regulatory T-cell lineage and an uncommitted minor population retaining plasticity. Proc Natl Acad Sci U S A 2009; 106(6): 1903-8.

125. Chapman-Shimshoni D, Yuklea M, Radnay J, Shapiro H, Lishner M. Simvastatin induces apoptosis of B-CLL cells by activation of mitochondrial caspase 9. Exp Hematol 2003; 31(9): 779-83.

126. Mevorach D. Systemic lupus erythematosus and apoptosis: a question of balance. Clin Rev Allergy Immunol 2003; 25(1): 49-60.

127. Dieker JW, Van Der Vlag J, Berden JH. Deranged removal of apoptotic cells: its role in the genesis of lupus. Nephrol Dial Transplant 2004; 19(2): 282-5.

128. Smeenk RJ. Antinuclear antibodies: cause of disease or caused by disease? Rheumatology (Oxford) 2000; 39(6): 581-4.

129. Grable-Esposito P, Katzberg HD, Greenberg SA, Srinivasan J, Katz J, Amato AA. Immune-mediated necrotizing myopathy associated with statins. Muscle Nerve 2010; 41(2): 185-90.

130. Christopher-Stine L, Casciola-Rosen LA, Hong G, Chung T, Corse AM, Mammen AL. A novel autoantibody recognizing $200-k d$ and $100-k d$ proteins is associated with an immune-mediated necrotizing myopathy. Arthritis Rheum 2010; 62(9): 2757-66.

131. Haas D, Hoffmann GF. Mevalonate kinase deficiency and autoinflammatory disorders. N Engl J Med 2007; 356(26): 2671-3.

132. Steffens S, Mach F. Drug insight: Immunomodulatory effects of statins--potential benefits for renal patients? Nat Clin Pract Nephrol 2006; 2(7): 378-87.

133. Schotte P, Denecker G, Van Den Broeke A, Vandenabeele P, Cornelis GR, Beyaert R. Targeting Rac1 by the Yersinia effector protein YopE inhibits caspase-1-mediated maturation and release of interleukin-1beta. J Biol Chem 2004; 279(24): 25134-42.

134. Coward WR, Marei A, Yang A, Vasa-Nicotera MM, Chow SC. Statin-induced proinflammatory response in mitogen-activated peripheral blood mononuclear cells through the activation of caspase-1 and IL-18 secretion in monocytes. J Immunol 2006; 176(9): 5284-92.

135. Lalor SJ, Dungan LS, Sutton CE, Basdeo SA, Fletcher JM, Mills KH. Caspase-1-processed cytokines IL-1beta and IL-18 promote IL-17 production by gammadelta and CD4 T cells that mediate autoimmunity. J Immunol 2011; 186(10): 5738-48.

136. Park SJ, Shin JI. Role of interleukin-12 and - 18 in lupus-like syndrome patients with statin use: comment on: Association between statin use and lupus-like syndrome using spontaneous reports. Semin Arthritis Rheum 2012; 41(6): e2-3.

137. Fairweather D. Autoimmune disease: mechanisms. In: Essential Immunology: Encyclopedia of Lifes Sciences. Delves PJ, Martin S, Burton DR, Roitt JM, eds. Hoboken: Wiley-Blackwell, 2007. p1-6.

138. Kivity S, Agmon-Levin N, Blank M, Shoenfeld Y. Infections and autoimmunity--friends or foes? Trends Immunol 2009; 30(8): 409-14.

139. Antoniou T, Zheng H, Singh S, Juurlink DN, Mamdani MM, Gomes T. Statins and the risk of herpes zoster: a population-based cohort study. Clin Infect Dis 2014; 58(3): 350-6.

140. Pirmohamed M. Statins, immunomodulation, and infections: a complex and unresolved relationship. Clin Infect Dis 2014; 58(3): 357-8.

141. Magulick JP, Frei CR, Ali SK, et al. The effect of statin therapy on the incidence of infections: a retrospective cohort analysis. Am J Med Sci 2014; 347(3): 211-6.

142. Vandenbroucke JP. Observational research, randomised trials, and two views of medical science. PLoS Med 2008; 5(3): e67.

143. Meyboom RH, Hekster YA, Egberts AC, Gribnau FW, Edwards IR. Causal or casual? The role of causality assessment in pharmacovigilance. Drug Saf 1997; 17(6): 374-89.

144. Meyboom RH. Causality assessment revisited. Pharmacoepidemiol Drug Saf 1998; 7 Suppl 1: S63-5.

145. Macedo AF, Marques FB, Ribeiro CF, Teixeira F. Causality assessment of adverse drug reactions: comparison of the results obtained from published decisional algorithms and from the evaluations of an expert panel, according to different levels of imputability. J Clin Pharm Ther 2003; 28(2): 137-43. 
146. Meyboom RH, Edwards IR. Rosuvastatin and the statin wars--the way to peace. Lancet 2004; 364(9450): 1997-9.

147. Wilson AM, Thabane L, Holbrook A. Application of data mining techniques in pharmacovigilance. $\mathrm{Br} \mathrm{J}$ Clin Pharmacol 2004; 57(2): 127-34

148. Bégaud B, Martin K, Haramburu F, Moore N. Rates of spontaneous reporting of adverse drug reactions in France. JAMA 2002; 288(13): 1588

149. De Boer A. When to publish measures of disproportionality derived from spontaneous reporting databases? Br J Clin Pharmacol 2011; 72(6): 909-11.

150. Schellevis FG, Westert GP, De Bakker DH. The actual role of general practice in the dutch health-care system. Results of the second dutch national survey of general practice. Med Klin (Munich) 2005; 100(10): 656-61.

151. Kennerfalk A, Ruigómez A, Wallander MA, Wilhelmsen L, Johansson S. Geriatric drug therapy and healthcare utilization in the United kingdom. Ann Pharmacother 2002; 36(5): 797-803.

152. Walley T, Mantgani A. The UK General Practice Research Database. Lancet 1997; 350(9084): 1097-9

153. Stirbu-Wagner I, Dorsman S, Visscher S, et al. [Landelijk Informatienetwerk Huisartsenzorg. Feiten en cijfers over huisartsenzorg in Nederland]. Utrecht/Nijmegen, The Netherlands: Nivel/IQ, 2010 [report in Dutch].

154. Preston C, Cheater F, Baker R, Hearnshaw H. Left in limbo: patients' views on care across the primary/ secondary interface. Qual Health Care 1999; 8(1): 16-21.

155. Kvamme OJ, Olesen F, Samuelson M. Improving the interface between primary and secondary care: a statement from the European Working Party on Quality in Family Practice (EQuiP). Qual Health Care 2001; 10(1): 33-9.

156. Schneeweiss S. Sensitivity analysis and external adjustment for unmeasured confounders in epidemiologic database studies of therapeutics. Pharmacoepidemiol Drug Saf 2006; 15(5): 291-303.

157. Sibbald B, Roland M. Understanding controlled trials. Why are randomised controlled trials important? BMJ 1998; 316(7126): 201.

158. Gardner TL, Dovey SM, Tilyard MW, Gurr E. Differences between prescribed and dispensed medications. NZ Med J 1996; 109(1017): 69-72.

159. Lau HS, de Boer A, Beuning KS, Porsius A. Validation of pharmacy records in drug exposure assessment. J Clin Epidemiol 1997; 50(5): 619-25.

160. Dasgupta B, Borg FA, Hassan N, et al. BSR and BHPR guidelines for the management of polymyalgia rheumatica. Rheumatology (Oxford) 2010; 49(1): 186-90

161. Petri M. Review of classification criteria for systemic lupus erythematosus. Rheum Dis Clin North Am 2005; 31(2): 245,54, vi

162. Lindquist M, Edwards IR. The WHO Programme for International Drug Monitoring, its database, and the technical support of the Uppsala Monitoring Center. J Rheumatol 2001; 28(5): 1180-7.

163. Thomas SL, Edwards CJ, Smeeth L, Cooper C, Hall AJ. How accurate are diagnoses for rheumatoid arthritis and juvenile idiopathic arthritis in the general practice research database? Arthritis Rheum 2008; 59(9): 1314-21.

164. Vandenbroucke JP. Oral contraceptives and rheumatoid arthritis. Lancet 1983; 2(8343): 228-9.

165. Chan KW, Felson DT, Yood RA, Walker AM. The lag time between onset of symptoms and diagnosis of rheumatoid arthritis. Arthritis Rheum 1994; 37(6): 814-20.

166. Delgado-Rodríguez M, Llorca J. Bias. J Epidemiol Community Health 2004; 58(8): 635-41.

167. Sathasivam S, Lecky B. Statin induced myopathy. BMJ 2008; 337: a2286.

168. Mansi I, Mortensen E. The controversy of a wider statin utilization: why? Expert Opin Drug Saf 2013; 12(3): 327-37.

169. Walker AM. Confounding by indication. Epidemiology 1996; 7(4): 335-6.

170. Grobbee DE, Hoes AW. Confounding and indication for treatment in evaluation of drug treatment for hypertension. BMJ 1997; 315(7116): 1151-4.

171. Libby P. Role of inflammation in atherosclerosis associated with rheumatoid arthritis. Am J Med 2008; 121(10 Suppl 1): S21-31. 
172. Van Halm VP, Nielen MM, Nurmohamed MT, et al. Lipids and inflammation: serial measurements of the lipid profile of blood donors who later developed rheumatoid arthritis. Ann Rheum Dis 2007; 66(2): 184-8.

173. Steiner G, Urowitz MB. Lipid profiles in patients with rheumatoid arthritis: mechanisms and the impact of treatment. Semin Arthritis Rheum 2009; 38(5): 372-81.

174. Alipour A, Van Oostrom AJ, Izraeljan A, et al. Leukocyte activation by triglyceride-rich lipoproteins. Arterioscler Thromb Vasc Biol 2008; 28(4): 792-7.

175. Van Oostrom AJ, Rabelink TJ, Verseyden C, Sijmonsma TP, Plokker HW, De Jaegere PP, Cabezas MC. Activation of leukocytes by postprandial lipemia in healthy volunteers. Atherosclerosis 2004; 177(1): 175-82.

176. Meijssen S, Van Dijk H, Verseyden C, Erkelens DW, Cabezas MC. Delayed and exaggerated postprandial complement component 3 response in familial combined hyperlipidemia. Arterioscler Thromb Vasc Biol 2002; 22(5): 811-6.

177. Hak E, Verheij TJ, Grobbee DE, Nichol KL, Hoes AW. Confounding by indication in non-experimental evaluation of vaccine effectiveness: the example of prevention of influenza complications. J Epidemiol Community Health 2002; 56(12): 951-5.

178. McMahon AD. Approaches to combat with confounding by indication in observational studies of intended drug effects. Pharmacoepidemiol Drug Saf 2003; 12(7): 551-8.

179. Klungel $\mathrm{OH}$, Martens EP, Psaty BM, et al. Methods to assess intended effects of drug treatment in observational studies are reviewed. J Clin Epidemiol 2004; 57(12): 1223-31.

180. Nurmohamed MT. Cardiovascular risk in rheumatoid arthritis. Autoimmun Rev 2009; 8(8): 663-7.

181. Nurmohamed MT, Dijkmans BA. Dyslipidaemia, statins and rheumatoid arthritis. Ann Rheum Dis 2009; 68(4): 453-5.

182. Perk J, De Backer G, Gohlke H, et al. European Guidelines on Cardiovascular Disease Prevention in Clinical Practice (version 2012). The Fifth Joint Task Force of the European Society of Cardiology and other societies on cardiovascular disease prevention in clinical practice (constituted by representatives of nine societies and by invited experts). G Ital Cardiol (Rome) 2013; 14(5): 328-92.

183. National Cholesterol Education Program (NCEP) Expert Panel on Detection, Evaluation, and Treatment of High Blood Cholesterol in Adults (Adult Treatment Panel III). Third Report of the National Cholesterol Education Program (NCEP) Expert Panel on Detection, Evaluation, and Treatment of High Blood Cholesterol in Adults (Adult Treatment Panel III) final report. Circulation 2002; 106(25): 3143-421.

184. Shah BR, Laupacis A, Hux JE, Austin PC. Propensity score methods gave similar results to traditional regression modeling in observational studies: a systematic review. J Clin Epidemiol 2005; 58(6): 550-9.

185. Stürmer T, Joshi M, Glynn RJ, Avorn J, Rothman KJ, Schneeweiss S. A review of the application of propensity score methods yielded increasing use, advantages in specific settings, but not substantially different estimates compared with conventional multivariable methods. J Clin Epidemiol 2006; 59(5): 437-47.

186. Collet JP, Boivin JF. Bias and confounding in pharmacoepidemiology. In: Strom BL, editor. Pharmacoepidemiology. $3^{\text {th }}$ ed. Chichester: John Wiley \& Sons Ltd.; 2000. p.765-84.

187. Lin DY, Psaty BM, Kronmal RA. Assessing the sensitivity of regression results to unmeasured confounders in observational studies. Biometrics 1998; 54(3): 948-63.

188. Velthove K. Markers for disease severity. Pharmacoepidemiological studies on obstructive lung disease (PhD Thesis). Utrecht, The Netherlands: Utrecht University 2010

189. Project Mondriaan. [Project Mondriaan Privacy. Trusted Third Party]. July 2011. Available from: http:// www.projectmondriaan.nl/privacy.html. [accessed 12 Apr 2014] [website in Dutch].

190. Elffers B, Verheij R. Verantwoording gegevenskoppeling Uit: NIVEL Zorgregistraties eerste lijn [internet]. December 2013. Available from: www.nivel.nl/node/3532. [accessed 24 Aug 2014] [website in Dutch].

191. Nguyen-Nielsen M, Svensson E, Vogel I, Ehrenstein V, Sunde L. Existing data sources for clinical epidemiology: Danish registries for studies of medical genetic diseases. Clin Epidemiol 2013; 5: 249-62.

192. Erichsen R, Lash TL, Hamilton-Dutoit SJ, Bjerregaard B, Vyberg M, Pedersen L. Existing data sources for clinical epidemiology: the Danish National Pathology Registry and Data Bank. Clin Epidemiol 2010; 2: 51-6.

193. Van Hunsel F, Härmark L, Pal S, Olsson S, van Grootheest K. Experiences with adverse drug reaction reporting by patients: an 11-country survey. Drug Saf 2012; 35(1): 45-60. 
194. The European Commission (EC). Regulation (EC) No 726/2004 of the European Parliament and of the Council. March 2004; Available from: http://ec.europa.eu/health/human-use/pharmacovigilance/index en.htm [accessed 23 December, 2013].

195. Watson R. New rules will allow EU patients to report drug concerns directly. BMJ 2010; 341: c5344.

196. Borg JJ, Aislaitner G, Pirozynski M, Mifsud S. Strengthening and rationalizing pharmacovigilance in the EU: where is Europe heading to? A review of the new EU legislation on pharmacovigilance. Drug Saf 2011; 34(3): 187-97.

197. Netherlands Pharmacovigilance Centre. [Bijwerking? Melden!]. April 2014; Available from: http://www. lareb.nl/Meld-bijwerking/Werkwijze-Lareb [accessed 18 April, 2014] [website in Dutch]

198. Rolfes L, Van Hunsel F, Van Puijenbroek E, Van Grootheest K. ADR reports of patients and healthcare professionals; how different are they? Pharmacoepidemiol Drug Saf 2013; 22: S315.

199. Blenkinsopp A, Wilkie $P$, Wang M, Routledge PA. Patient reporting of suspected adverse drug reactions: a review of published literature and international experience. Br J Clin Pharmacol 2007; 63(2): 148-56.

200. Alsheikh Ali A, Ambrose M, Kuvin J, Karas R. The safety of rosuvastatin as used in common clinical practice: a postmarketing analysis. Circulation 2005; 111(23): 3051-7.

201. Jellinger PS, Smith DA, Mehta AE, et al. American Association of Clinical Endocrinologists' Guidelines for Management of Dyslipidemia and Prevention of Atherosclerosis. Endocr Pract 2012; 18 Suppl 1: 1-78.

202. National Collaborating Centre for Primary Care. Lipid modification: cardiovascular risk assessment and the modification of blood lipids for the primary and secondary prevention of cardiovascular disease. NICE clinical guideline 67. London, UK: National Institute for Health and Clinical Excellence, 2008 (reissued 2010).

203. Goff DC Jr, Lloyd-Jones DM, Bennett G, et al. 2013 ACC/AHA Guideline on the Assessment of Cardiovascular Risk: A Report of the American College of Cardiology/American Heart Association Task Force on Practice Guidelines. Circulation 2013 Nov 12. [Epub ahead of print].

204. British Cardiac Society, British Hypertension Society, Diabetes UK, HEART UK, Primary Care Cardiovascular Society, Stroke Association. JBS 2: Joint British Societies' guidelines on prevention of cardiovascular disease in clinical practice. Heart 2005; 91 Suppl 5: v1-52.

205. Wiersma T, Smulders YM, Stehouwer CD, Konings KT, Lanphen J. [Summary of the multidisciplinary guideline on cardiovascular risk management (revision 2011)]. Ned Tijdschr Geneeskd 2012; 156(36):A5104 [article in Dutch].

206. Summary of product characteristics Advisory Group. A guideline on summary of product characteristics (SmPC). Brussels, Belgium: European Commission, Enterprise and Industry Directorate-General, 2009.

207. Mammen AL, Chung T, Christopher-Stine L, Rosen P, Rosen A, Doering KR, Casciola-Rosen LA. Autoantibodies against 3-hydroxy-3-methylglutaryl-coenzyme A reductase in patients with statin-associated autoimmune myopathy. Arthritis Rheum 2011; 63(3): 713-21.

208. Mammen AL, Pak K, Williams EK, Brisson D, Coresh J, Selvin E, Gaudet D. Rarity of anti-3-hydroxy-3-methylglutaryl-coenzyme A reductase antibodies in statin users, including those with self-limited musculoskeletal side effects. Arthritis Care Res (Hoboken) 2012; 64(2): 269-72.

209. Dutch Biobanking and Biomolecular Resource Research Infrastructure (BBMRI-NL). Managing resources for the future of biomedical research. Rainbow Projects. January 2014. Available from: http:// www.bbmri.nl/en-gb/activities/rainbow-projects [accessed 14 March, 2014].

210. Etminan M. Pharmacoepidemiology II: the nested case-control study--a novel approach in pharmacoepidemiologic research. Pharmacotherapy 2004; 24(9): 1105-9.

211. Lévesque LE, Hanley JA, Kezouh A, Suissa S. Problem of immortal time bias in cohort studies: example using statins for preventing progression of diabetes. BMJ 2010; 340: b5087. 


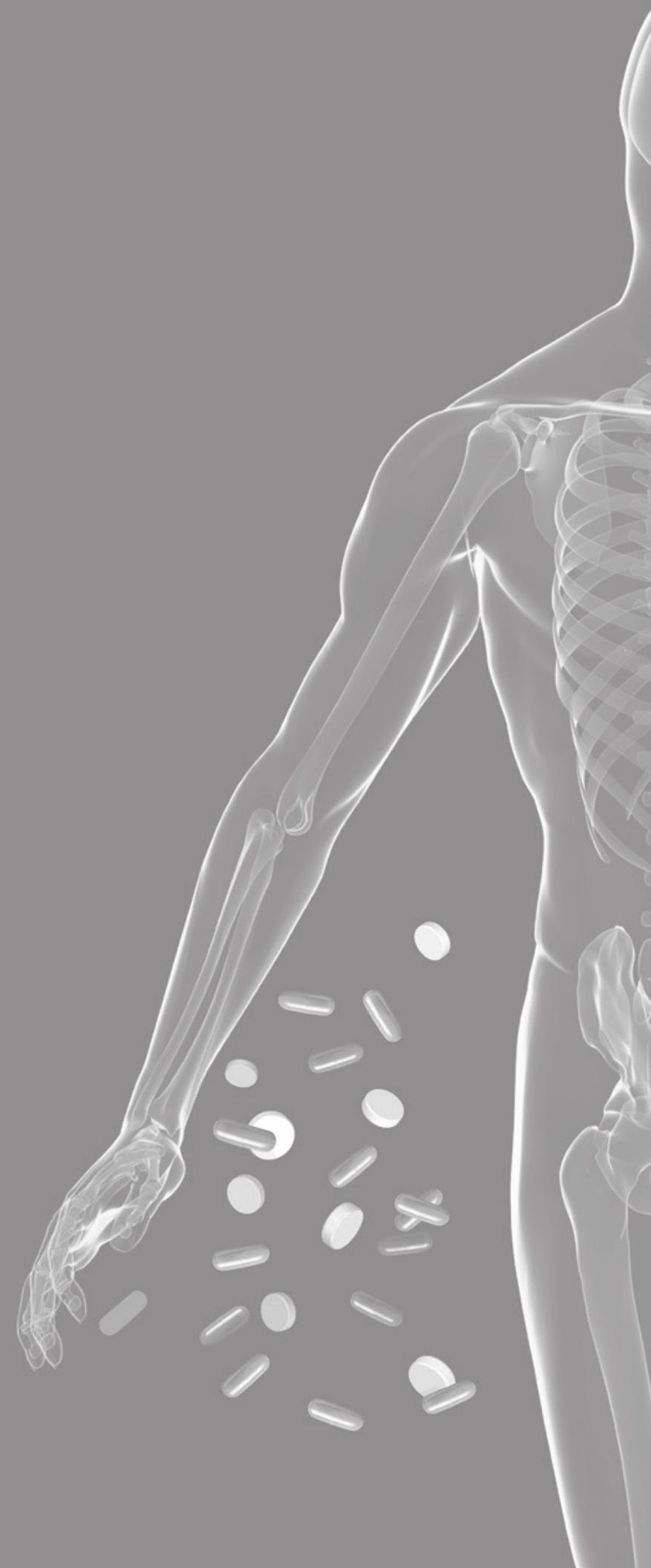




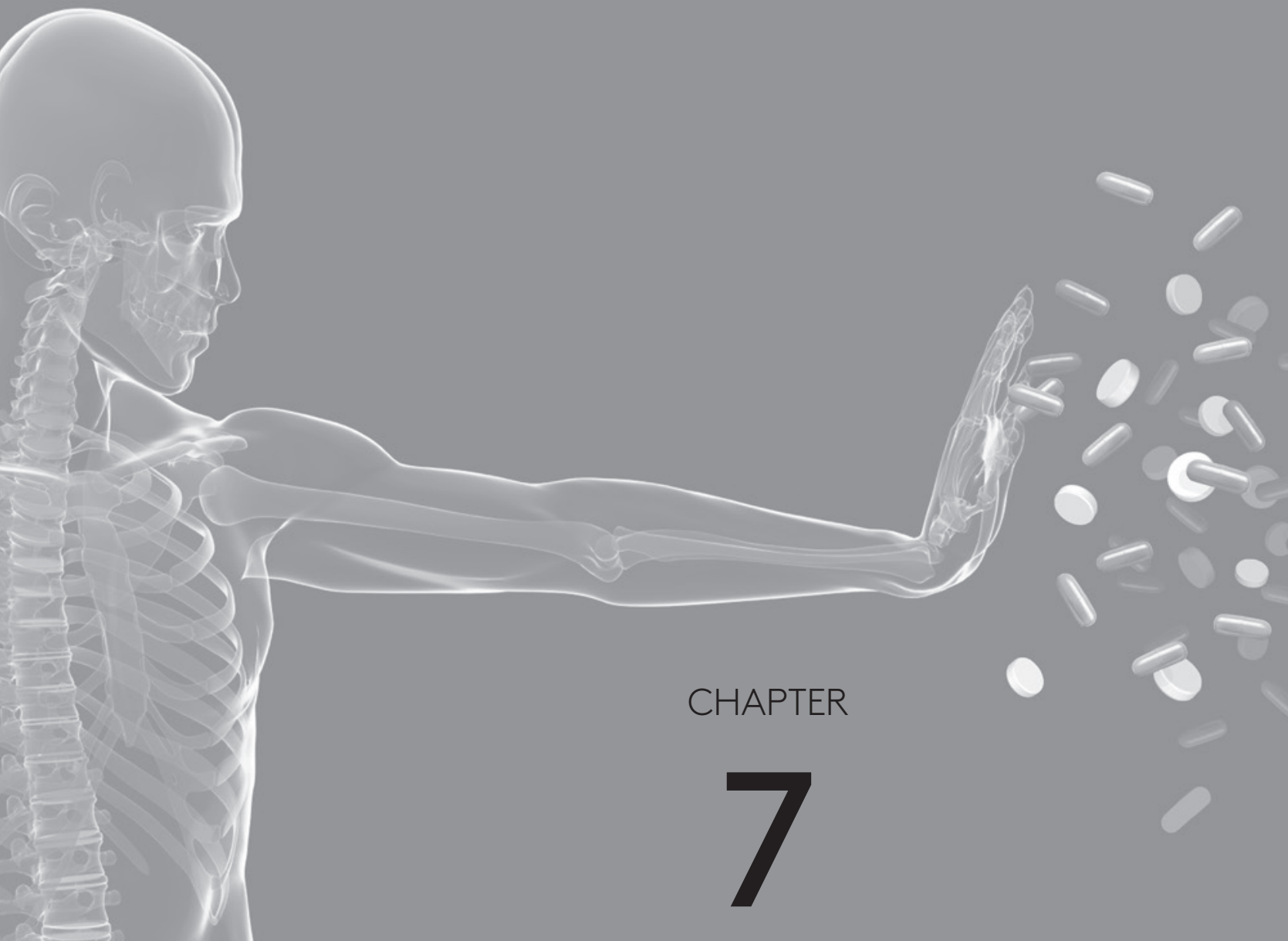

VALORISATION ADDENDUM 



\section{INTRODUCTION}

Several studies have demonstrated that environmental components play a major role in this process of autoimmunity ${ }^{1-5}$ and may be responsible for the increased prevalence of autoimmune disorders in highly industrialised countries ${ }^{6}$. Many drugs are associated with the development of certain autoimmune disorders, e.g., lupus-like syndrome and vasculitis ${ }^{7-19}$.

Statins, angiotensin converting enzyme (ACE) inhibitors and angiotensin receptor blockers (ARBs) are widely prescribed to reduce the risk of cardiovascular morbidity and mortality in patients with or at risk for coronary heart disease 20-25. In addition to their cholesterol and blood pressure lowering effects, several studies have shown that these drugs also have anti-inflammatory and immunomodulatory properties ${ }^{26-43}$, and are thereby also effective in the treatment of certain autoimmune disorders. The beneficial effects of these drugs are well described, but interestingly, such immunomodulating effects may, on the other hand, adversely influence immune regulation. Therefore, we hypothesise that statins, ACE inhibitors and ARBs facilitate the development of autoimmunity, potentially resulting in autoimmune disorders. In this thesis, we have examined whether there is an association between the use of cardiovascular drugs (statins, ACE inhibitors and ARBs) and the incidence of autoimmune diseases.

Based on the findings reported in this thesis we recommend several implications for daily practice. The implications for daily practice are:

Reporting adverse drug reactions by health care professionals and patients Inform physicians and pharmacists of statin-associated autoimmune disorders Physicians should be conservative in prescribing statins in low cardiovascular risk patients

Update drug labels for statins

Develop a validated risk tool to predict statin-associated autoimmune disorder All these implications are described in detail below.

\section{Reporting adverse drug reactions by health care professionals and patients}

Despite the pro-active and routine approaches used in pharmacovigilance, underreporting of the ADRs is extremely high and expected to be approximately $90 \% 44$. Additionally, selective underreporting may have occurred since the reporter may not always be alert on the ADR, and therefore; the assessment of an association between drug intake and an adverse event remains a challenge. However, collection of ADRs is warranted for signal detection (hypothesis strengthening) and hypothesis generation. In this thesis, we have shown that two decades after approval for marketing, reporting of lupus-like syndrome and PMR occurred more often with statins than with other drugs 
We urge physicians, pharmacists and other healthcare providers, and above all, patients to report potential side effects to pharmacovigilance centres. Patients have been invited to report ADRs directly in the US, Canada and New Zealand 45. Since the European Parliament legislative resolution on pharmacovigilance of medicinal products for human use (Regulation [EC] No 726/2004) which was launched in July 2012 46, patients from all European member states are allowed to report concerns regarding drugs, directly to the competent authorities by web-based structured forms 47,48. At the Dutch pharmacovigilance centre, Lareb, every ADR from patients is stored in a database and compared with previous national and international ADRs. All potential ADR signals are sent to the Dutch regulatory authority and European Medicines Agency 49.

The information reported by patients and healthcare professionals is generally comparable, although patients' reports on ADRs tend to provide more information on the impact of the reported ADRs whereas reports from healthcare professionals focus on diagnosis related information. Both types of information are valuable to pharmacovigilance centres 50,51 . In studies using databases containing spontaneously reported ADRs, we have shown that excluding ADRs submitted by patients did not change our findings (chapters 2.1 and 2.2), indicating that ADRs submitted by patients are useful for signal detection and hypothesis generation.

\section{Inform physicians and pharmacists of statin-associated autoimmune disorders}

In general, statins are considered to be safe although the market withdrawal of cerivastatin has demonstrated that some serious adverse effects are not always detected in clinical trials. The most commonly reported side effects of statins are marked elevation of liver enzymes and muscular abnormalities ${ }^{52}$. Our findings raise awareness about the possibility of developing lupus-like syndrome, PMR or RA in patients treated with statins. For instance, when a patient on statin therapy presents with myalgia of hip and shoulder region in combination with morning stiffness, one should consider the possibility of PMR. Physicians and pharmacists should be informed about these possible statin-associated autoimmune disorders.

\section{Physicians should be conservative in prescribing statins in low cardiovascular risk patients}

According to several guidelines, patients with a high risk of cardiovascular disease should be treated with statins 53-59. For instance, according to the European guideline on cardiovascular disease prevention in clinical practice, patients with a 10-year risk of cardiovascular disease death of $\geq 5 \%$ (based on the Systematic Coronary Risk Evaluation Project (SCORE) chart) should be on statin treatment ${ }^{53}$. In patients with a low cardiovascular risk (SCORE level of $\leq 5 \%$ ), statin therapy may be considered 
when lifestyle modification such as smoking cessation, regular physical activity and/ or dietary changes is not sufficient 53 . However, the findings in this thesis shed new light on the benefit-risk ratio of statins. We found that statin use was associated with an increased occurrence or risk of developing lupus-like syndrome, PMR or RA. Our findings may slightly alter the 'risk' component of the benefit-risk ratio. However, others have not corroborated these findings ${ }^{60-64}$, and therefore; more definitive studies in healthy (low-risk) subjects are needed. Until this question is resolved, one should be conservative in prescribing statins to individuals with a low cardiovascular risk.

\section{Update drug labels for statins}

In daily clinical practice, drug labels can play a pivotal role in signalling the risk of rare autoimmune disorders. Drug labels inform healthcare professionals on the safe and effective use of a specific drug. According to the guideline for the summary of product characteristics 65 , a list of all adverse reactions with their respective frequency and the source of the safety database (e.g. from clinical trials, post-authorisation safety studies or spontaneous reporting) should be enclosed. So far, data on statin-associated immune-mediated disorders has only been based on case reports. In this thesis, we have conducted several studies with different designs (e.g. observational, experimental, case history) and data sources (e.g. spontaneous reporting, electronic health records, laboratory and in vivo), and all of them showed an increased occurrence or risk of developing autoimmune disorders when individuals were treated with statins. Based on this information, we suggest that it is time to update the information on the label.

\section{Develop a validated risk tool to predict statin-associated autoimmune disorders}

Despite the increase in statin use in last two decades, only a small number of patients who were treated with these drugs over a prolonged period of time developed a systemic statin-induced syndrome ${ }^{8}$. While the absolute number of patients is low, statin-associated autoimmune disorders may potentially impose a heavy burden on public health, since these disorders can be chronic, with long-term morbidity and may account for high healthcare costs, loss of quality of life and loss of productivity. Therefore, careful risk assessment should be considered. Patients who are susceptible to autoimmune disorders may have a high risk of developing these disorders when treated with a statin. A validated risk prediction tool, such as developing biomarkers may therefore be useful to predict statin-associated autoimmune disorders. Recently, autoantibodies, that recognise 3-hydroxy-3-methylglutaryl-coenzyme A reductase (HMGCR) have been identified in patients with statin-associated autoimmune myopathy 66 , whereas in a large population of statin exposed patients without myopathy no anti-HMGCR antibodies were found 67 . Although to date, no validated biomarkers 
are available for predicting statin-associated autoimmune disorders, we propose that such risk prediction tools could potentially help healthcare professionals to categorise patients based on risk profiles. 


\section{REFERENCES}

1. Molina V, Shoenfeld Y. Infection, vaccines and other environmental triggers of autoimmunity. Autoimmunity 2005; 38(3): 235-45

2. Marrack P, Kappler J, Kotzin BL. Autoimmune disease: why and where it occurs. Nat Med 2001; 7(8): 899-905.

3. Klareskog L, Malmström V, Lundberg K, Padyukov L, Alfredsson L. Smoking, citrullination and genetic variability in the immunopathogenesis of rheumatoid arthritis. Semin Immunol 2011; 23(2): 92-8.

4. Singh N, Singh U, Singh B, Price R, Nagarkatti M, Nagarkatti P. Activation of aryl hydrocarbon receptor (AhR) leads to reciprocal epigenetic regulation of FoxP3 and IL-17 expression and amelioration of experimental colitis. PLoS ONE 2011; 6(8): e23522.

5. Todd J. D'oh! genes and environment cause Crohn's disease. Cell 2010; 141(7): 1114-6.

6. WHO Collaborating Centre for Immunotoxicology and Allergic Hypersensitivity. Principles and methods for assessing autoimmunity associated with exposure to chemicals: Environmental Health Criteria. Bilthoven, The Netherlands: World Health Organisation, 2006.

7. Wiik A. Drug-induced vasculitis. Curr Opin Rheumatol 2008; 20(1): 35-9

8. Vasoo S. Drug-induced lupus: an update. Lupus 2006; 15(11): 757-61.

9. Vedove CD, Del Giglio M, Schena D, Girolomoni G. Drug-induced lupus erythematosus. Arch Dermatol Res 2009; 301(1): 99-105.

10. Olsen N. Drug-induced autoimmunity. Best Pract Res Clin Rheumatol 2004; 18(5): 677-88

11. Mor A, Pillinger M, Wortmann R, Mitnick H. Drug-induced arthritic and connective tissue disorders. Semin Arthritis Rheum 2008; 38(3): 249-64.

12. Mongey A, Hess E. Drug insight: autoimmune effects of medications-what's new? Nat Clin Pract Rheumatol 2008; 4(3): 136-44.

13. Borchers AT, Keen CL, Gershwin ME. Drug-induced lupus. Ann N Y Acad Sci 2007; 1108: 166-82.

14. Choi HK, Merkel PA, Walker AM, Niles JL. Drug-associated antineutrophil cytoplasmic antibody-positive vasculitis: prevalence among patients with high titers of antimyeloperoxidase antibodies. Arthritis rheumatism 2000; 43(2): 405-13.

15. Dolman KM, Gans RO, Vervaat TJ, et al. Vasculitis and antineutrophil cytoplasmic autoantibodies associated with propylthiouracil therapy. Lancet 1993; 342(8872): 651-2.

16. Hess E. Drug-related lupus. N Engl J Med 1988; 318(22): 1460-2.

17. Marzano AV, Vezzoli P, Crosti C. Drug-induced lupus: an update on its dermatologic aspects. Lupus 2009; 18(11): 935-40

18. Rubin RL. Drug-induced lupus. Toxicology 2005; 209(2): 135-47.

19. Uetrecht J. Current trends in drug-induced autoimmunity. Autoimmun Rev 2005; 4(5): 309-14.

20. Law MR, Wald NJ, Rudnicka AR. Quantifying effect of statins on low density lipoprotein cholesterol, ischaemic heart disease, and stroke: systematic review and meta-analysis. BMJ 2003; 326(7404): 1423.

21. PROGRESS Collaborative Group. Randomised trial of a perindopril-based blood-pressure-lowering regimen among 6,105 individuals with previous stroke or transient ischaemic attack. Lancet 2001; 358(9287): 1033-41.

22. MacMahon S, Peto R, Cutler J, et al. Blood pressure, stroke, and coronary heart disease. Part 1, Prolonged differences in blood pressure: prospective observational studies corrected for the regression dilution bias. Lancet 1990; 335(8692): 765-74

23. Collins R, Peto R, MacMahon S, et al. Blood pressure, stroke, and coronary heart disease. Part 2, Short-term reductions in blood pressure: overview of randomised drug trials in their epidemiological context. Lancet 1990; 335(8693): 827-38.

24. Antiplatelet Trialists' Collaboration. Collaborative overview of randomised trials of antiplatelet therapy--I: Prevention of death, myocardial infarction, and stroke by prolonged antiplatelet therapy in various categories of patients. BMJ 1994; 308(6921): 81-106.

25. Antithrombotic Trialists' Collaboration. Collaborative meta-analysis of randomised trials of antiplatelet therapy for prevention of death, myocardial infarction, and stroke in high risk patients. BMJ 2002; 324(7329): 71-86 
26. Platten M, Youssef S, Hur EM, et al. Blocking angiotensin-converting enzyme induces potent regulatory T cells and modulates TH1- and TH17-mediated autoimmunity. Proc Natl Acad Sci U S A 2009; 106(35): 14948-53.

27. Sukumaran V, Watanabe K, Veeraveedu PT, et al. Olmesartan, an AT1 antagonist, attenuates oxidative stress, endoplasmic reticulum stress and cardiac inflammatory mediators in rats with heart failure induced by experimental autoimmune myocarditis. Int J Biol Sci 2011; 7(2): 154-67.

28. Ruiz-Ortega M, Bustos C, Hernández-Presa MA, Lorenzo O, Plaza JJ, Egido J. Angiotensin II participates in mononuclear cell recruitment in experimental immune complex nephritis through nuclear factor-kappa B activation and monocyte chemoattractant protein-1 synthesis. J Immunol 1998; 161(1): 430-9

29. Schindler R, Dinarello CA, Koch KM. Angiotensin-converting-enzyme inhibitors suppress synthesis of tumour necrosis factor and interleukin 1 by human peripheral blood mononuclear cells. Cytokine 1995; 7(6): 526-33.

30. Fukuzawa M, Satoh J, Sagara M, et al. Angiotensin converting enzyme inhibitors suppress production of tumor necrosis factor-alpha in vitro and in vivo. Immunopharmacology 1997; 36(1): 49-55.

31. Constantinescu CS, Goodman DB, Ventura ES. Captopril and lisinopril suppress production of interleukin-12 by human peripheral blood mononuclear cells. Immunol Lett 1998; 62(1): 25-31.

32. Martin MF, Surrall KE, McKenna F, Dixon JS, Bird HA, Wright V. Captopril: a new treatment for rheumatoid arthritis? Lancet 1984; 1(8390): 1325-8.

33. Perry ME, Chee MM, Ferrell WR, Lockhart JC, Sturrock RD. Angiotensin receptor blockers reduce erythrocyte sedimentation rate levels in patients with rheumatoid arthritis. Ann Rheum Dis 2008; 67(11): 1646-7

34. Ridker PM, Danielson E, Fonseca FA, et al. Reduction in C-reactive protein and LDL cholesterol and cardiovascular event rates after initiation of rosuvastatin: a prospective study of the JUPITER trial. Lancet 2009; 373(9670): 1175-82

35. Weitz Schmidt G, Welzenbach K, Brinkmann V, et al. Statins selectively inhibit leukocyte function antigen-1 by binding to a novel regulatory integrin site. Nat Med 2001; 7(6): 687-92.

36. Youssef S, Stüve O, Patarroyo JC, et al. The HMG-CoA reductase inhibitor, atorvastatin, promotes a Th2 bias and reverses paralysis in central nervous system autoimmune disease. Nature 2002; 420(6911): 78-84.

37. Vollmer T, Key L, Durkalski V, et al. Oral simvastatin treatment in relapsing-remitting multiple sclerosis. Lancet 2004; 363(9421): 1607-8.

38. Neuhaus O, Stüve O, Zamvil SS, Hartung HP. Are statins a treatment option for multiple sclerosis? Lancet Neurol 2004; 3(6): 369-71.

39. McCarey DW, Mclnnes IB, Madhok R, et al. Trial of Atorvastatin in Rheumatoid Arthritis (TARA): double-blind, randomised placebo-controlled trial. Lancet 2004; 363(9426): 2015-21.

40. Leung BP, Sattar N, Crilly A, et al. A novel anti-inflammatory role for simvastatin in inflammatory arthritis. J Immunol 2003; 170(3): 1524-30.

41. Kwak BR, Mulhaupt F, Mach F. Atherosclerosis: anti-inflammatory and immunomodulatory activities of statins. Autoimmun Rev 2003; 2(6): 332-8.

42. Arnaud $\mathrm{C}$, Braunersreuther V, Mach F. Toward immunomodulatory and anti-inflammatory properties of statins. Trends Cardiovasc Med 2005; 15(6): 202-6.

43. López-Pedrera C, Aguirre MÃ, Barbarroja N, Cuadrado MJ. Accelerated atherosclerosis in systemic lupus erythematosus: role of proinflammatory cytokines and therapeutic approaches. J Biomed Biotechnol 2010; 2010: pii:607084.

44. Hazell L, Shakir SA. Under-reporting of adverse drug reactions: a systematic review. Drug Saf 2006; 29(5): 385-96.

45. Van Hunsel F, Härmark L, Pal S, Olsson S, van Grootheest K. Experiences with adverse drug reaction reporting by patients: an 11-country survey. Drug Saf 2012; 35(1): 45-60.

46. The European Commission (EC). Regulation (EC) No 726/2004 of the European Parliament and of the Council. March 2004; Available from: http://ec.europa.eu/health/human-use/pharmacovigilance/ index_en.htm [accessed 23 December, 2013]. 
47. Watson R. New rules will allow EU patients to report drug concerns directly. BMJ 2010; 341: c5344.

48. Borg JJ, Aislaitner G, Pirozynski M, Mifsud S. Strengthening and rationalizing pharmacovigilance in the EU: where is Europe heading to? A review of the new EU legislation on pharmacovigilance. Drug Saf 2011; 34(3): 187-97.

49. Netherlands Pharmacovigilance Centre. [Bijwerking? Melden!]. April 2014; Available from: http://www. lareb.nl/Meld-bijwerking/Werkwijze-Lareb [accessed 18 April, 2014] [website in Dutch].

50. Rolfes L, Van Hunsel F, Van Puijenbroek E, Van Grootheest K. ADR reports of patients and healthcare professionals; how different are they? Pharmacoepidemiol Drug Saf 2013; 22: S315.

51. Blenkinsopp A, Wilkie P, Wang M, Routledge PA. Patient reporting of suspected adverse drug reactions: a review of published literature and international experience. $\mathrm{Br} \mathrm{J}$ Clin Pharmacol 2007; 63(2): 148-56.

52. Alsheikh Ali A, Ambrose M, Kuvin J, Karas R. The safety of rosuvastatin as used in common clinical practice: a postmarketing analysis. Circulation 2005; 111(23): 3051-7.

53. Perk J, De Backer G, Gohlke H, et al. European Guidelines on Cardiovascular Disease Prevention in Clinical Practice (version 2012). The Fifth Joint Task Force of the European Society of Cardiology and other societies on cardiovascular disease prevention in clinical practice (constituted by representatives of nine societies and by invited experts). G Ital Cardiol (Rome) 2013; 14(5): 328-92.

54. Jellinger PS, Smith DA, Mehta AE, et al. American Association of Clinical Endocrinologists' Guidelines for Management of Dyslipidemia and Prevention of Atherosclerosis. Endocr Pract 2012; 18 Suppl 1: 1-78.

55. National Collaborating Centre for Primary Care. Lipid modification: cardiovascular risk assessment and the modification of blood lipids for the primary and secondary prevention of cardiovascular disease. NICE clinical guideline 67. London, UK: National Institute for Health and Clinical Excellence, 2008 (reissued 2010).

56. Goff DC Jr, Lloyd-Jones DM, Bennett G, et al. 2013 ACC/AHA Guideline on the Assessment of Cardiovascular Risk: A Report of the American College of Cardiology/American Heart Association Task Force on Practice Guidelines. Circulation 2013 Nov 12. [Epub ahead of print].

57. British Cardiac Society, British Hypertension Society, Diabetes UK, HEART UK, Primary Care Cardiovascular Society, Stroke Association. JBS 2: Joint British Societies' guidelines on prevention of cardiovascular disease in clinical practice. Heart 2005; 91 Suppl 5: v1-52.

58. National Cholesterol Education Program (NCEP) Expert Panel on Detection, Evaluation, and Treatment of High Blood Cholesterol in Adults (Adult Treatment Panel III). Third Report of the National Cholesterol Education Program (NCEP) Expert Panel on Detection, Evaluation, and Treatment of High Blood Cholesterol in Adults (Adult Treatment Panel III) final report. Circulation 2002; 106(25): 3143-421.

59. Wiersma T, Smulders YM, Stehouwer CD, Konings KT, Lanphen J. [Summary of the multidisciplinary guideline on cardiovascular risk management (revision 2011)]. Ned Tijdschr Geneeskd 2012; 156(36):A5104 [article in Dutch].

60. Chodick G, Amital H, Shalem Y, Kokia E, Heymann AD, Porath A, Shalev V. Persistence with statins and onset of rheumatoid arthritis: a population-based cohort study. PLoS Med 2010; 7(9): e1000336.

61. Jick SS, Choi H, Li L, Mclnnes IB, Sattar N. Hyperlipidaemia, statin use and the risk of developing rheumatoid arthritis. Ann Rheum Dis 2009; 68(4): 546-51.

62. Hippisley-Cox J, Coupland C. Unintended effects of statins in men and women in England and Wales: population based cohort study using the QResearch database. BMJ 2010; 340: c2197.

63. Smeeth L, Douglas I, Hall AJ, Hubbard R, Evans S. Effect of statins on a wide range of health outcomes: a cohort study validated by comparison with randomized trials. Br J Clin Pharmacol 2009; 67(1): 99-109.

64. Schmidt T, Battafarano DF, Mortensen EM, Frei CR, Mansi I. Frequency of development of connective tissue disease in statin-users versus nonusers. Am J Cardiol 2013; 112(6): 883-8.

65. Summary of product characteristics Advisory Group. A guideline on summary of product characteristics (SmPC). Brussels, Belgium: European Commission, Enterprise and Industry Directorate-General, 2009.

66. Mammen AL, Chung T, Christopher-Stine L, Rosen P, Rosen A, Doering KR, Casciola-Rosen LA. Autoantibodies against 3-hydroxy-3-methylglutaryl-coenzyme A reductase in patients with statin-associated autoimmune myopathy. Arthritis Rheum 2011; 63(3): 713-21. 
67. Mammen AL, Pak K, Williams EK, Brisson D, Coresh J, Selvin E, Gaudet D. Rarity of anti-3-hydroxy3-methylglutaryl-coenzyme A reductase antibodies in statin users, including those with self-limited musculoskeletal side effects. Arthritis Care Res (Hoboken) 2012; 64(2): 269-72 


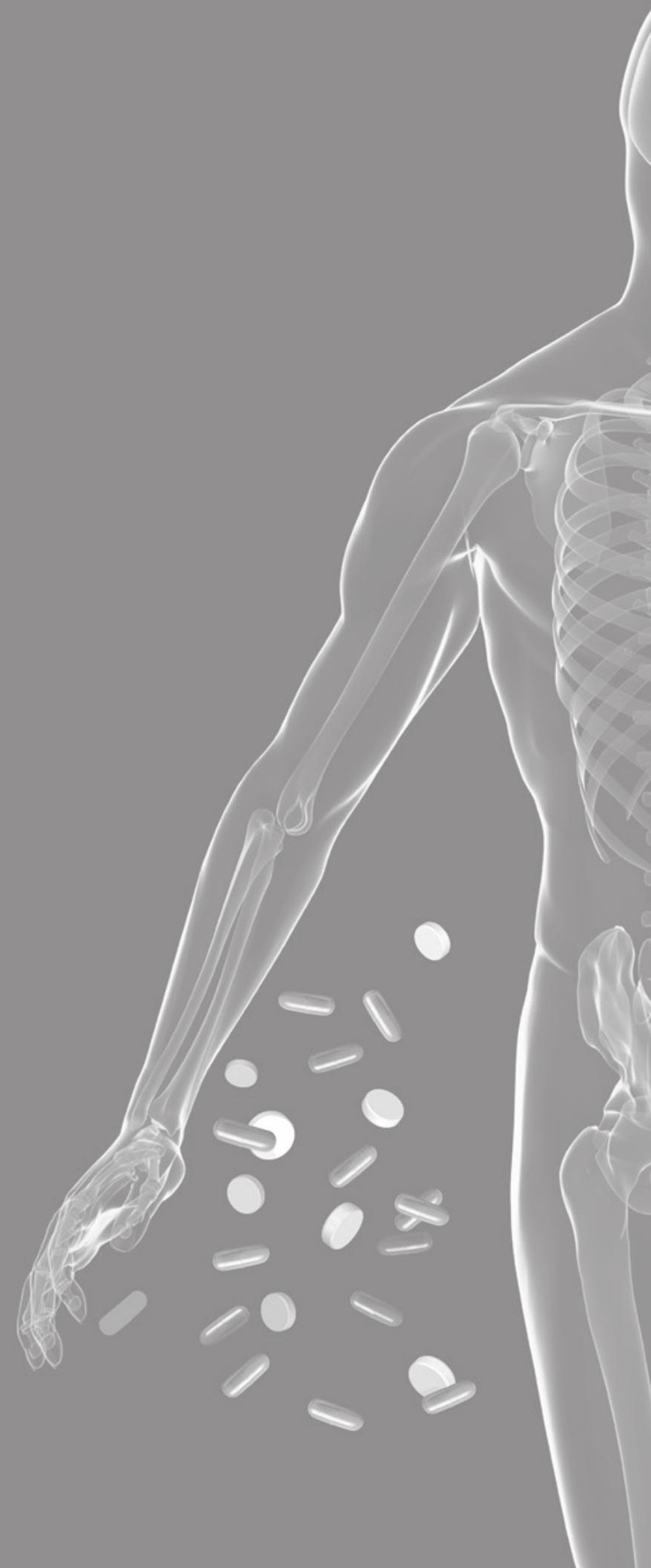




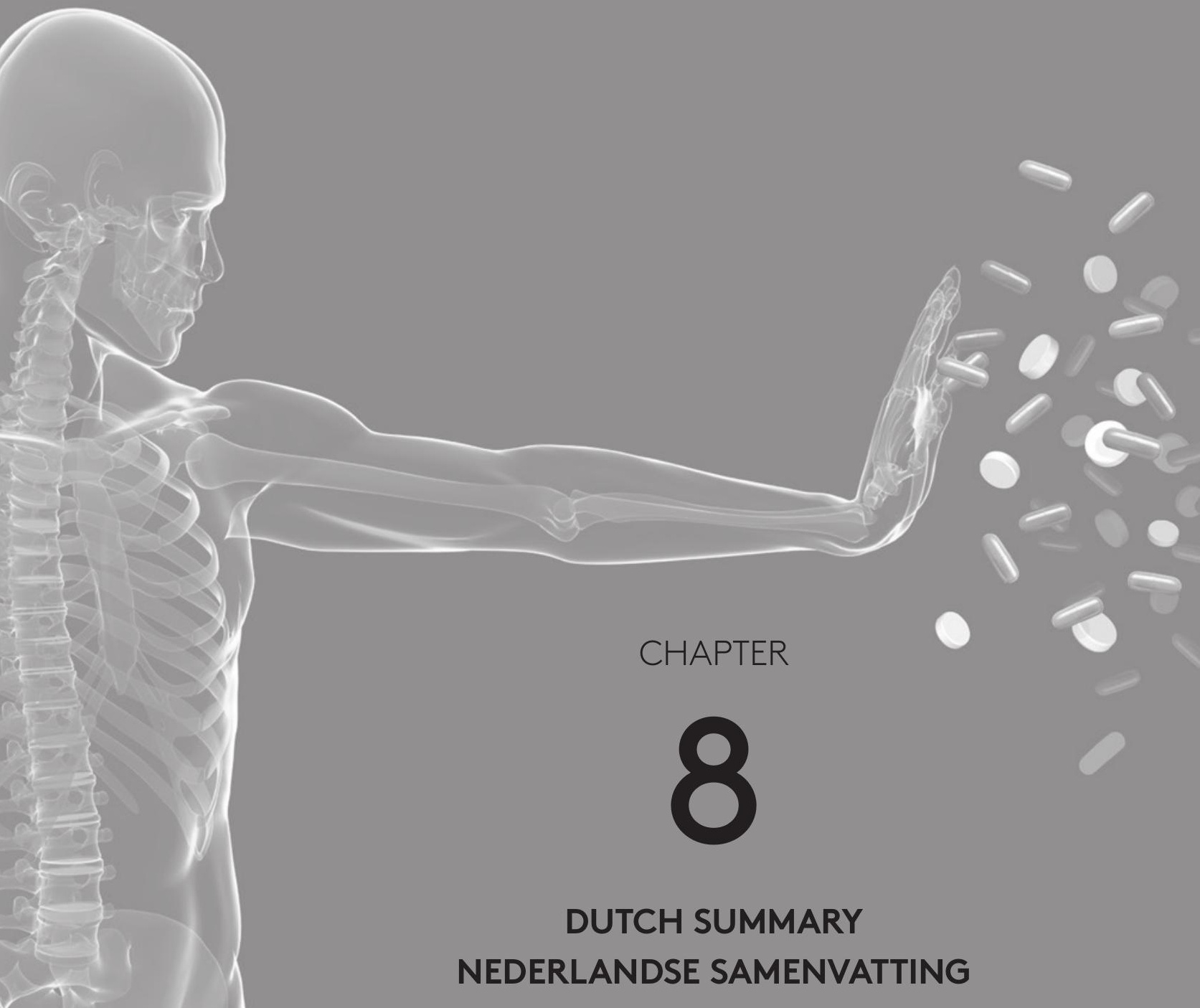





\section{NEDERLANDSE SAMENVATTING}

Het immuunsysteem is een verdedigingssysteem met als doel om indringers, zoals virussen, bacteriën, parasieten, tumorcellen, of veranderde eigen cellen te bestrijden. Wanneer het immuunsysteem niet meer het verschil kan maken tussen de indringers en het lichaamseigen weefsel, valt het immuunsysteem de lichaamseigen cellen aan. We spreken dan van auto-immuniteit. De ontstekingsziekten die hierbij ontstaan noemt men auto-immuunziekten. Momenteel zijn er meer dan 80 ziekten die tot de familie van auto-immuunziekten behoren. Voorbeelden van auto-immuunziekten zijn reumatoïde artritis (RA), systemische lupus erythematodes (SLE), diabetes type I en auto-immuun schildklierziekten.

Het ontstaan van auto-immuniteit is een proces dat bestaat uit meerdere factoren, waaronder erfelijke- en omgevingsfactoren. Ondanks vele jaren van uitgebreid onderzoek is het exacte mechanisme dat verantwoordelijk is voor het ontstaan van auto-immuniteit nog niet opgehelderd. Meerdere onderzoeken laten zien dat de omgevingsfactoren zoals roken, voeding, blootstelling aan geneesmiddelen en/of chemische stoffen een belangrijke rol spelen in het ontstaan van auto-immuniteit. De omgevingsfactoren zijn mogelijk verantwoordelijk voor de grote aantallen autoimmuunziekten in de Westerse wereld. Uit verscheidene onderzoeken is gebleken dat bepaalde geneesmiddelen gerelateerd zijn aan het ontwikkelen van autoimmuunziekten. De cholesterol- en bloeddruk verlagende geneesmiddelen: statines, angiotensine I converterend enzym (ACE) remmers en angiotensine receptor blokkers (ARB's) worden wereldwijd voorgeschreven aan patiënten die nog geen hart- en vaatziekten hebben doorgemaakt, maar die wel een verhoogd risico op harten vaatziekten (primaire preventie) hebben. Ook worden deze medicijnen voorgeschreven aan patiënten die al bekend zijn met hart- en vaatziekten (secundaire preventie). Naast de cholesterol- en bloeddruk verlagende effecten, laten veel onderzoeken zien dat deze drie geneesmiddelen ook ontstekingsremmende en immuun-modulerende (effect op het immuunsysteem) eigenschappen hebben. Deze eigenschappen kunnen effectief zijn in het behandelen van bepaalde auto-immuunziekten. De behandelingseffecten van statines, ACE remmers of ARB's zijn uitvoerig in de literatuur beschreven. De immuun-modulerende eigenschappen van deze drie geneesmiddelen kunnen naast de behandelingseffecten ook een ongewenste invloed hebben op het immuunsysteem. Daarom veronderstellen we dat statines, ACE remmers en ARB's mogelijk de ontwikkeling van auto-immuniteit vergemakkelijken, wat zou kunnen resulteren in een auto-immuunziekte.

Op het moment van toelating van een geneesmiddel op de markt is niet alles bekend over de veiligheid van het geneesmiddel. Pas jaren nadat het geneesmiddel op de markt gebracht is, kan het volledige veiligheidsprofiel verkregen worden. Dit gebrek aan kennis kan mogelijk liggen aan de tekortkomingen van een klinische trial. Een 
klinische trial is een wetenschappelijke methode om te bepalen of een behandeling met bijvoorbeeld een geneesmiddel werkt. Mogelijke tekortkomingen van klinische trials kunnen zijn dat ze een te kleine groep patiënten bevatten of alleen patiënten bevatten die nagenoeg dezelfde karakteristieken en medische geschiedenis hebben. Een andere oorzaak kan zijn dat ze een beperkte duur hebben en er geen rekening wordt gehouden met hoe het geneesmiddel in de dagelijkse praktijk wordt gebruikt door de patiënten. In de praktijk blijkt het ook nog eens een uitdaging te zijn om een auto-immuun gerelateerde bijwerking op te sporen, aangezien deze bijwerkingen vrij zeldzaam zijn, ze pas na langdurig geneesmiddelen gebruik verschijnen en niet altijd verdwijnen na het stoppen met het geneesmiddel.

In dit proefschrift onderzoeken wij of er een relatie is tussen het gebruik van geneesmiddelen voor hart- en vaatziekten (statines, ACE remmers en ARB's ) en het risico op het ontwikkelen van auto-immuunziekten. Om onze hypothese kracht bij te zetten hebben we drie onderzoeken uitgevoerd met gegevens uit een database van spontaan gerapporteerde bijwerkingen tijdens het gebruik van statines in de dagelijkse praktijk (hoofdstuk 2). In hoofdstuk 2.1 hebben we door middel van een case (een specifieke bijwerking) /non-case (alle andere bijwerkingen) onderzoeksopzet, onderzocht of er een relatie is tussen statinegebruik en de aanwezigheid van lupusachtig syndroom. In dit onderzoek vonden we dat het statinegebruik vaker was gerapporteerd in patiënten met een lupusachtig syndroom dan in patiënten die een andere bijwerking hadden doorgemaakt. Deze bevinding ligt in lijn met een eerder uitgevoerd onderzoek, dat gebruik maakte van gegevens uit de Franse bijwerkingen database. Door dezelfde onderzoeksopzet te gebruiken, zijn we in hoofdstuk 2.2 nagegaan of er een relatie is tussen statinegebruik en de aanwezigheid van polymyalgia rheumatica (PMR). Ook in dit onderzoek vonden we dat statinegebruik vaker was gerapporteerd in patiënten met een PMR dan in patiënten die een andere bijwerking hadden doorgemaakt. Verder waren er zes individuele veiligheidsrapporten waarin PMR als een bijwerking na herhaald gebruik van een statine werd gerapporteerd. Deze bevindingen versterken de verdenking van een oorzakelijk verband tussen statinegebruik en de aanwezigheid van PMR. Onze bevindingen uit hoofdstuk 2.1 en 2.2 komen overeen met de casussen die staan beschreven in de literatuur, wat een aanwijzing suggereert (signaaldetectie) van statinegerelateerde lupusachtige syndroom en PMR. Om onze bevinding van statinegerelateerd PMR kracht bij te zetten, hebben we de gegevens van één patiënt opgevraagd en in detail in een rapport beschreven (hoofdstuk 2.3). Bij deze patiënt zagen we na het stoppen met statinegebruik dat PMR was verdwenen. Echter, toen de patiënt opnieuw werd behandeld met statines, verschenen de symptomen van PMR wederom met daarbij arteriitis temporalis (reuscelarteriitis), een ziekte die zeer nauw verbonden is met PMR. Op basis van dit patiëntenrapport hebben we mogelijk het oorzakelijke verband van statinegerelateerd PMR/arteriitis temporalis weergegeven. Het gebruik van een 
database van spontaan gerapporteerde bijwerkingen is voornamelijk gericht op het versterken van de hypothese en niet op het testen van een hypothese.

We hebben onze hypothese getest door gebruik te maken van databases met elektronische medische patiëntengegevens. In hoofdstuk 3 hebben we twee onderzoeken uitgevoerd om te bepalen of er een relatie is tussen statinegebruik en een verhoogd risico op het ontwikkelen van RA. In hoofdstuk 3.1 hebben we de gegevens over statinegebruik en de eerste diagnose van RA afgeleid van de gegevens uit het Landelijk Informatie Netwerk Huisartszorg (LINH). In deze database staan van de patiënten de voorgeschreven medicatie, ziekten en verwijzingen naar specialisten beschreven. Door het uitvoeren van een patiënt(case)-controle-onderzoek vonden we een verhoogd risico op RA in patiënten die zes maanden lang statines gebruikten. Daarnaast zagen we een kleine trend tussen een toegenomen potentie (kracht) van de statines en het risico op het ontwikkelen van RA. Er werd door ons geen trend gevonden in de duur van gebruik, aanbevolen dagelijkse dosis (ADD) en het aantal voorgeschreven recepten. Een patiënt-controle-onderzoek heeft een aantal tekortkomingen. Om deze tekortkomingen te beperken, hebben we een tweede onderzoek uitgevoerd naar de relatie tussen statinegebruik en het risico op het ontwikkelen van RA. In het tweede onderzoek, hoofdstuk 3.2, hebben we gebruik gemaakt van een tijdsafhankelijke analyse, wat betekent dat het statinegebruik in de tijd wordt uitgezet en gevolgd. In dit onderzoek hebben we niet alleen de onderzoeksresultaten uit hoofdstuk 3.1 willen bevestigen, maar ook willen uitbreiden. Dit is gedaan met behulp van een patroon-analyse van de risico's op het ontwikkelen van RA door de veranderingen in statinegebruik gedurende de tijd. De gegevens van het statinegebruik en de eerste diagnose van RA komen voort uit de recepten, diagnose van ziekten en verwijzingen naar specialisten. Deze patiëntengegevens zijn opgeslagen in de Engelse Clinical Practice Research Datalink (CPRD) database. Ook in een grote groep patiënten (cohort), waar we terug kijken naar het verleden van deze patiënten (retrospectief onderzoek), zien we opnieuw dat statinegebruik is gerelateerd aan een verhoogd risico op het ontwikkelen van RA. Daarnaast zien we dat het risico op RA aanzienlijk is verhoogd in het eerste jaar na de start van het statinegebruik en dat daarna de relatie tussen statinegebruik en het risico op het ontwikkelen van RA is verdwenen. Wanneer we de statine-gebruikers indelen in nieuwe gebruikers en voormalige gebruikers vinden we dezelfde resultaten. Omdat het risico op het ontwikkelen van RA kort na de start van het statinegebruik was verhoogd, kunnen statines mogelijk als een katalysator werken voor het ontstaan van RA in patiënten die gevoelig zijn voor het ontwikkelen van RA. We hebben dit aangetoond in een proefdieronderzoek, dat later zal worden besproken. In een onderzoek van verscheidene patiëntenrapporten van statinegerelateerde lupusachtig syndroom, laat het grootste deel van deze patiënten zien dat de periode tot het ontstaan van lupusachtige syndroom minder dan een jaar is. Hieruit voortvloeiend, 
denken wij dat het langdurig gebruik van statines in patiënten die niet gevoelig zijn voor het ontwikkelen van RA als veilig kan worden ervaren. Daarnaast zien we in dezelfde studie (hoofdstuk 3.2) alleen de relatie tussen statinegebruik en een verhoogd risico op het ontwikkelen van RA in hergebruikers van statines en vrouwen. Tot op heden zijn er vijf andere studies uitgevoerd die de relatie tussen statinegebruik en het ontwikkelen van RA hebben onderzocht. Deze vijf studies laten geen eenduidig resultaat zien, maar géén van deze studies toont een verhoogd risico op het ontwikkelen van RA na statinegebruik. Een mogelijke verklaring voor het verschil in de resultaten van onze studies (hoofdstuk 3.1 en 3.2) met de andere vijf studies kan zijn dat we geen latency (reactie)-tijd in ons onderzoek hebben meegenomen. Ook al gebruikten wij twee verschillende onderzoeksopzetten en databases in hoofdstuk 3.1 en 3.2, de verdeling van patiënten met hart- en vaatziekten en de daarbij behorende risicofactoren (bijvoorbeeld hyperlipidemie (een verzamelnaam voor uiteenlopende stoornissen in de vetstofwisseling)) waren niet gelijk verdeeld tussen de twee onderzochte groepen. In hoofdstuk 3.1 vergeleken we de patiënten met RA met de controles en in hoofdstuk 3.2 vergeleken we de statine-gebruikers met de niet-gebruikers.

We hebben onze hypothese verder getest door na te gaan of het gebruik van ACE remmers of ARB's gerelateerd is aan het risico op het ontwikkelen van RA. In dit patiënt-controle-onderzoek namen we alleen gebruikers van ACE remmers of ARB's en andere bloeddruk verlagende geneesmiddelen uit de Nederlandse huisartsen database (LINH) mee (hoofdstuk 3.3). Er werd geen relatie tussen ACE remmers of ARB's en het risico op het ontwikkelen van RA waargenomen. De resultaten waren hetzelfde wanneer we de blootstelling aan ACE remmers of ARB's hadden ingedeeld in huidig en voormalig gebruik, duur- of zelfs dosering van het gebruik. Deze resultaten ondersteunen niet de hypotheses dat ACE remmers of ARB's, RA op gang kunnen brengen of juist kunnen beschermen tegen RA. Voor zover wij weten is er, uitgezonderd ons onderzoek, geen enkel onderzoek dat de relatie tussen ACE remmers of ARB's en het ontwikkelen van RA heeft bestudeerd. Bewijs voor de relatie tussen deze twee geneesmiddelen en de aanwezigheid van auto-immuunziekten komt voort uit patiëntenrapporten, proefdierstudies en patiënten met een bepaalde auto-immuunziekte. Deze studies laten tegengestelde resultaten zien. In onze studie laten we het risico op RA zien in patiënten die geen medische geschiedenis hebben van RA maar die wel zijn behandeld met ACE remmers of ARB's. Hypertensie (een hoge bloeddruk) kan worden gezien als een aandoening waarbij het immuunsysteem een rol speelt en dit is meer aanwezig in RA patiënten dan in de algemene bevolking. Met betrekking tot de immuun-modulerende eigenschappen en de hoge aanwezigheid van hypertensie in RA patiënten, kunnen behandelingen met ACE remmers of ARB's effectief zijn in patiënten met hypertensie en RA. 
In de introductie van het proefschrift wordt beschreven dat de statines de ontwikkeling van auto-immuniteit kunnen vergemakkelijken. Er wordt algemeen gedacht dat statines, naast een ontstekingsremmende werking, ook een directe immuun-modulerende werking hebben op T-cellen. T-cellen zijn afweercellen die verantwoordelijk zijn voor de cellulaire immuniteit. Cellulaire immuniteit is een immuunreactie die gericht is op het onschadelijk maken van virussen en bacteriën. Eén van de typen T-cellen zijn de T-helper cellen die vervolgens weer onderverdeeld zijn in onder andere T-helper (Th) 1 en Th2 cellen. Statines kunnen ook een verschuiving van Th1 naar Th2 immuunreactie bevorderen, met als gevolg een ontregeling in de immuun homeostase. Homeostase is het in evenwicht zijn van allerlei functies (zoals temperatuur- en zuurgraad) en het vermogen van het lichaam om dit evenwicht te behouden, ondanks omgevingsinvloeden. In de voorgaande studies konden we de bovenstaande hypothese over het directe effect van de statines op T-cellen niet bestuderen, maar doordat we gebruik hebben kunnen maken van sera (het bloed zonder bloedcellen) uit de Doetinchem Cohort Studie in combinatie met de uitgiften van statines uit PHARMO-RLS, een database die alle uitgiften van geneesmiddelen van apothekers bijhoudt, kan het nu wel. In hoofdstuk 4 hebben we de effecten van statinegebruik op de aangeboren ("innate") en verworven ("adaptive") afweer (immuniteit) en zelfs de tolerantie van het immuunsysteem onderzocht. De effecten van het statinegebruik op het immuunsysteem werden bepaald door het meten van serum concentraties van het acute fase eiwit (C-reactieve protein (CRP)), neopterine en immunoglobuline (lg) E. Daarnaast werd er getest op de aanwezigheid van de autoantilichamen, antinucleaire antilichamen (ANA) en reumatoïde factor (RF). Het onderzoek werd uitgevoerd onder personen uit de algemene bevolking die al of niet waren blootgesteld aan statines. In verscheidene studies werd al aangetoond dat statinegebruik de concentratie CRP kan verlagen. In de lijn der verwachting, waren de concentraties CRP lager in de statine-gebruikers dan in de niet-gebruikers. Verder vonden we in de statine-gebruikers een trend naar een hogere concentratie IgE antilichamen, dat is een merkstof voor een Th2 immuunreactie. Er werden geen verschillen waargenomen in de concentraties neopterine, een merkstof voor een Th1 immuunreactie, en de aanwezigheid van de autoantilichamen tussen de statine-gebruikers en niet-gebruikers. Onze bevindingen suggereren dat de statines een ontstekingsremmende werking hebben, maar er is niet voldoende bewijs dat de statines een effect uitoefenen op een Th1 immuunreactie en/of het verlies van zelf-tolerantie van het immuunsysteem. Een immuun-modulerend effect op een Th2 immuunreactie kan echter niet worden uitgesloten. Onze bevinding van de Th2 reactie komt overeen met twee eerdere studies die beide aantonen dat statines een Th2 immuunreactie bevorderen. Aangezien we geen effect van het statinegebruik op de aanwezigheid van autoantilichamen hebben gevonden, lijkt het erop dat statines zelf geen defect in de immuuntolerantie veroorzaken, maar ze kunnen mogelijk een bestaande 
auto-immuun gevoelige conditie laten ontwikkelen tot het openbaren van een klinische ziekte.

Gebaseerd op de bevindingen die gepresenteerd zijn in hoofdstuk 3, vinden we een relatie tussen statinegebruik en een verhoogd risico op het ontwikkelen van RA. Echter, onze bevindingen zijn niet bevestigd in andere studies. Om het oorzakelijke verband tussen statinegebruik en het risico op het ontwikkelen van RA te onderzoeken, hebben we een proefdierstudie uitgevoerd (hoofdstuk 5). In deze proefdierstudie, is een specifiek muismodel gebruikt, dat karakteristiek is voor RA. De muizen werden dagelijks via het voedsel gedurende 4 weken behandeld met één van de twee typen statines (pravastatine of atorvastatine), of met een zoutoplossing, voor of na de opwekking van artritis. We vonden dat een behandeling met $40 \mathrm{mg} / \mathrm{kg}$ pravastatine of $10 \mathrm{mg} / \mathrm{kg}$ atorvastatine, voor of na het opwekken van artritis resulteerde in een vervroegde artritis. Dit in vergelijking met geen behandeling met statines. Daarnaast zagen we dat een behandeling met atorvastatine of pravastatine na de het opwekken van artritis, resulteerde in een vroegere aanvang van artritis dan behandeling met dezelfde statines vóór artritis opwekking. De bevinding van een vroegere aanvang van artritis na behandeling met statines vóór de opwekking van artritis, ondersteunt de bevindingen uit hoofdstuk 3.1 en 3.2. Vier andere proefdierstudies die hetzelfde muismodel hebben gebruikt, laten gunstige of geen effecten van behandelingen met statines op de aanvang van artritis zien. Deze studies gebruiken echter verschillende types, trajecten en doses, start en duur van het statinegebruik. Dit betekent dat het moeilijk is om de resultaten van deze vier studies met onze resultaten te vergelijken. Desondanks ondersteunen onze bevindingen een oorzakelijk verband tussen statinegebruik en het risico op het ontwikkelen van RA.

In hoofdstuk 6 zijn de belangrijkste bevindingen van de in dit proefschrift onderzochte relaties tussen statines, ACE remmers en ARB's en het risico op het ontwikkelen van een aantal auto-immuunziekten beschreven en bediscussieerd in het licht van de bestaande literatuur. Dit is zowel vanuit immunologisch als epidemiologisch perspectief uitgevoerd. We hebben verscheidene immunologische verklaringen gegeven voor onze bevindingen. Eén van de verklaringen is dat we geen effect van statinegebruik op de aanwezigheid van autoantilichamen hebben gevonden, wat er op lijkt te duiden dat statines zelf geen defect in de immuuntolerantie kunnen veroorzaken, maar mogelijk een bestaande auto-immuun gevoelige conditie kunnen laten ontwikkelen tot een klinische ziekte. Vervolgens zijn er tal van methodologische beschouwingen over de onderzoeksopzetten, de definitie van het gebruik van statines, ACE remmers en ARBs en auto-immuunziekten beschreven, en worden ook andere methodologische beperkingen in dit proefschrift bediscussieerd, waaronder 'protopathic bias', 'ascertainment bias', 'detectie bias', verschillen in het voorschrijfgedrag van huisartsen, 'confounding by indication' en het ontbreken van verstorende variabelen ("confounders"). Deze methodologische beperkingen 
hebben zeker invloed gehad op onze bevindingen. Eén van de discussiepunten is het gebruik van de juiste definitie voor auto-immuunziekten en de verstorende variabelen. Door het verkrijgen van een complete medische geschiedenis van de patiënt, door bijvoorbeeld het koppelen van verscheidene datasets, kan de misclassificatie van het lupusachtig syndroom, PMR of RA, of 'protopathic bias' worden verkleind. Een ander belangrijk punt is dat statines, ACE remmers of ARB's kunnen dienen als een soort maatstaf voor hyperlipidemie of hypertensie. Meer onderzoek zal moeten worden uitgevoerd om na te gaan of bijvoorbeeld hyperlipidemie of de immuun-modulerende eigenschappen van statines voornamelijk verantwoordelijk is voor de verhoogde kans op het ontwikkelen van auto-immuunziekten.

Ten slotte is er een eindconclusie getrokken over de bevindingen die in dit proefschrift zijn gepresenteerd. Samenvattend zagen we dat statinegebruik is gerelateerd aan een verhoogd risico op het ontwikkelen van auto-immuunziekten. Huidig bewijs laat zien dat statines zijn gerelateerd aan een verhoogde aanwezigheid van, of risico op het ontwikkelen van lupusachtig syndroom, PMR of RA. Het risico op het ontwikkelen van RA is verhoogd in het eerste jaar na de start van het gebruik. Bovendien is er een oorzakelijk verband tussen statinegebruik en het risico op auto-immuunziekten aangetoond. Anderzijds was er geen relatie tussen statinegebruik en het verlies van zelf-tolerantie van het immuunsysteem onder personen uit de algemene bevolking. In tegenstelling tot het gebruik van statines kan het gebruik van ACE remmers and ARB's als veilig worden ervaren, zelfs na langdurig gebruik. 


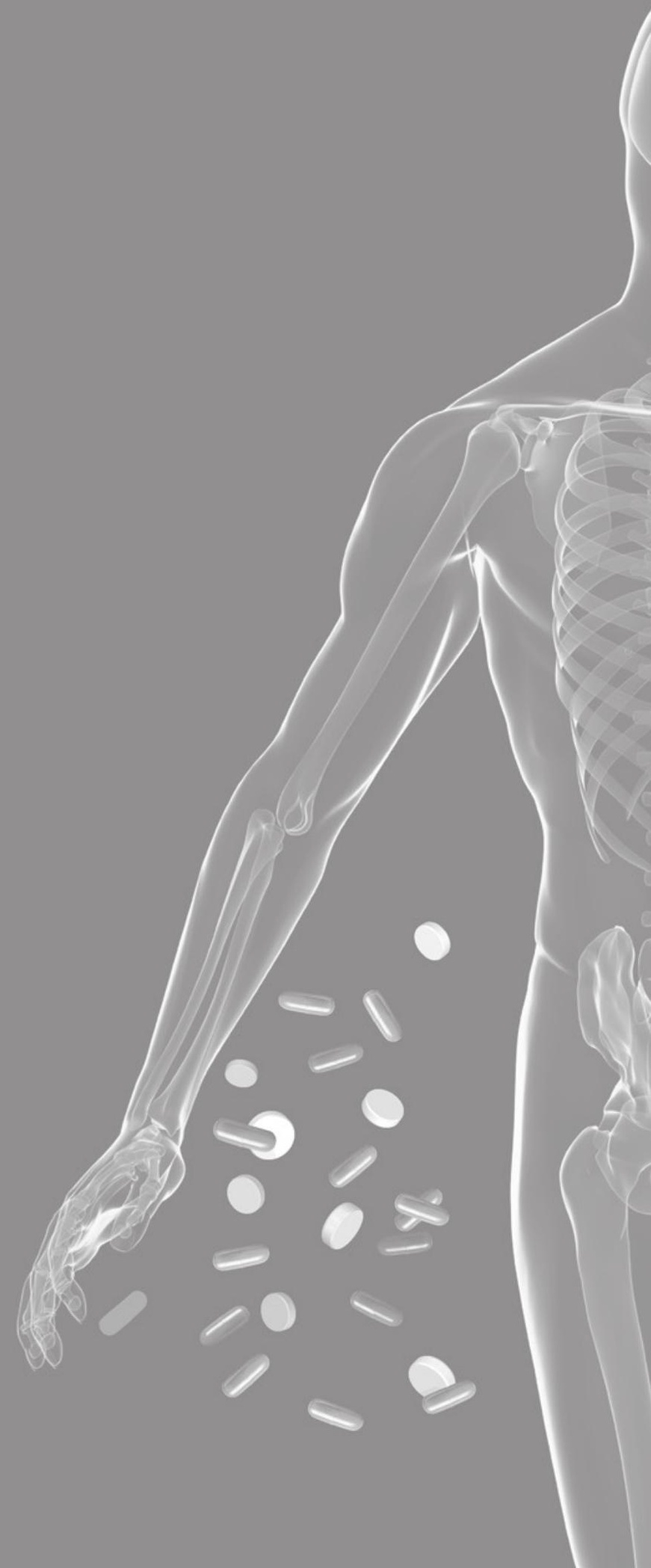




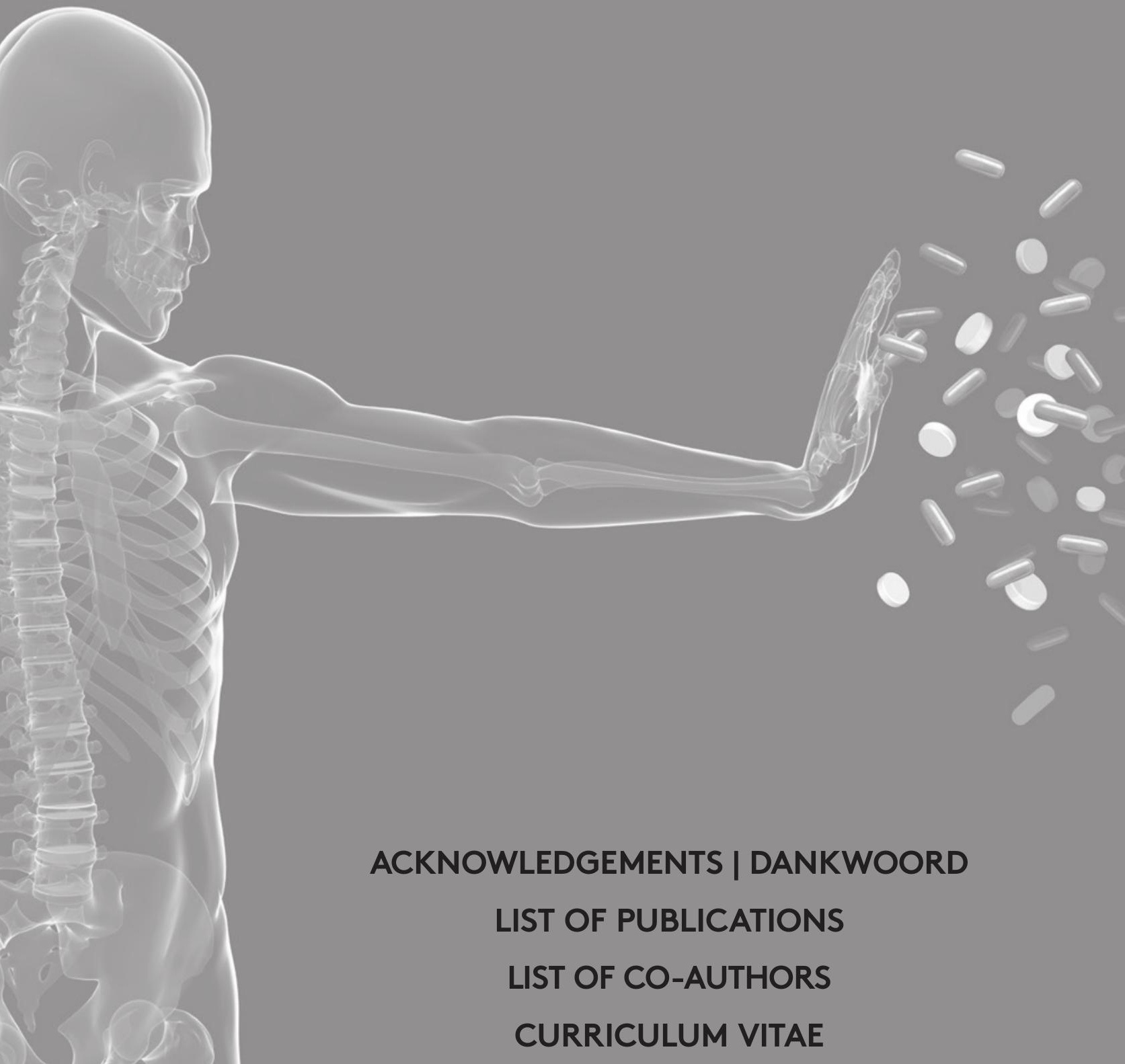





\section{DANKWOORD}

Het is dan eindelijk af! Een proefschrift schrijven doe je natuurlijk niet alleen. Velen hebben op verschillende wijzen een bijdrage geleverd aan de totstandkoming van dit proefschrift. In het bijzonder wil ik graag de volgende mensen bedanken.

Allereerst wil ik mijn promotoren, Prof. dr. H. van Loveren en Prof. dr. JW Cohen Tervaert, en mijn copromotoren, dr. R.J. Vandebriel en dr. O.H. Klungel, bedanken. Beste Henk, graag wil ik jou danken voor de kans die je me hebt geboden om mijn promotie te starten en te voltooien. In het bijzonder wil ik jou bedanken voor de vrijheid en de verantwoordelijkheden die ik van jou kreeg om in dit project nieuwe studies en de daarbij behorende samenwerkingen op te zetten. Ondanks deze mate van vrijheid hield je altijd wel de vinger aan de pols over de invulling van het proefschrift. Beste Jan Willem, jouw praktijkgerichte inbreng gaf vanaf het begin af aan, een enorme impuls aan het project. Jouw enthousiasme, bevlogenheid en positieve instelling heeft me altijd geboeid en gaf me veel energie. Samenwerken met jou heb ik als zeer inspirerend ervaren. Niet alleen tijdens het promotieonderzoek was je van onschatbare waarde, maar je hebt ook een grote rol gespeeld bij het tot stand brengen van de samenwerking tussen de Universiteit Maastricht en University of British Columbia $(\mathrm{UBC})$. Met deze samenwerking heb ik mijn postdoctorale positie bij UBC kunnen behouden. Daar ben ik jou ontzettend dankbaar voor. Ik ben erg blij dat we onze samenwerking verder kunnen voortzetten.

Beste Rob, ondanks onze verschillende wetenschappelijke achtergronden, heb ik jouw rol in mijn promotieonderzoek erg gewaardeerd. Voornamelijk bij het opzetten en het uitwerken van het proefdieronderzoek. Zonder jouw hulp was dit onderzoek niet tot stand gekomen. Verder kon ik altijd rekenen op je snelle en kritische reactie op mijn manuscripten.

Beste Olaf, al vrij snel in het promotieonderzoek kwamen we er achter dat de datasets niet toereikend waren voor het bestuderen van onze onderzoeksvraag. Door jouw netwerk heb je me kennnis laten maken met verschillende nationale en internationale onderzoeksgroepen. Deze onderzoeksgroepen konden ons voorzien van datasets die passend waren voor onze onderzoeksvraag. Daarnaast heb ik jouw kennis in de epidemiologie en je kritische blik op mijn manuscripten zeer gewaardeerd.

Naast mijn promotieteam heb ik de nodige hulp van anderen gehad, waarvoor mijn dank.

Prof. dr. H.M.G. Leufkens en dr. J-W. van der Laan, beste Bert en Jan-Willem, jullie stonden niet alleen aan de wieg van dit onderzoeksproject, maar bleven me tijdens het onderzoek ook met goede raad en advies bijstaan. Bedankt! 
Dr. R.H.B. Meijboom, beste Ron, bedankt voor je bijdrage aan de twee studies met de WHO database en bij het uitwerken van één specifieke melding uit de WHO database tot een casus. Het was een erg leerzaam proces om tot de resultaten te komen die in de hoofdstukken 2.1, 2.2 en 2.3 zijn beschreven. Door jouw samenwerking kreeg ik de kans om ook een kijkje te nemen in de keuken van de farmacovigilantie. Dank daarvoor! Ook dr. J.M.L. Passier, beste Anneke, bedankt voor het verstrekken van informatie ten behoeve van het Nederlandse registratiesysteem voor de bijwerkingen van geneesmiddelen. I would also like to thank Kristina Star for her help with the WHO database and her critical notes to the manuscripts presented in chapter 2.1 and 2.2 of this thesis. Furthermore, I would like to thank prof. dr. L. Niskanen and dr. Markku for providing me with patient information for the case report as described in chapter 2.3. Last but not least, with regards to the studies using WHO database, I would like to thank 'my student' Siti Rizny Saldi for her support in the data analyses, as presented in chapter 2.1 and 2.2. In addition, thank you for helping me out with the data analysis for chapter 3.3. Congratulations on your position as an epidemiologist at the Centre for Clinical Epidemiology and Evidence-Based Medicine in Jakarta, Indonesia!

Mijn speciale dank gaat uit naar Liset van Dijk. Beste Liset, jouw input op de twee studies met de LINH data (hoofdstuk 3.1 en 3.3) waren zeer waardevol voor mij. Jouw enthousiasme, doortastendheid en opbouwende feedback tijdens onze besprekingen zorgden ervoor dat ik weer met een frisse blik aan de slag kon gaan. Daarnaast volgde je op de voet de voortgang van mijn promotieonderzoek. Na mijn officiële promotietraject bood je mij een tijdelijke positie als farmaco-epidemioloog aan bij het NIVEL. Naast de werkzaamheden voor het NIVEL, gaf je me ook de ruimte om mijn promotieonderzoek af te ronden en maakte je het mogelijk om mijn droom te verwezenlijken. Met jouw creativiteit en vastberadenreid werd er een samenwerking opgezet met UBC waardoor ik voor de eerste keer de grote overstap kon maken naar Vancouver, Canada. Hier ben ik jou nog altijd heel erg dankbaar voor.

Mijn dank is groot aan prof. dr. T.P. van Staa. Beste Tjeerd, in de laatste fase van mijn promotieonderzoek kreeg ik zowaar de kans om met jou samen te werken. Ik ben je nog steeds dankbaar dat jij mijn uitkomsten van de auto-immuunziekten in jouw protocol opnam. Hierdoor mocht ik gebruik maken van jouw speciaal gecreëerde cohort van de CPRD data dat uiteindelijk heeft geresulteerd in een studie, zoals staat beschreven in hoofdstuk 3.2. Dr. A. Lalmohamed en dr. F. de Vries, beste Arief en Frank, bedankt voor jullie ondersteuning bij de interpretatie van de CPRD data en het programmeren in SAS. 
Dr. P.C. Souverein, beste Patrick, waar zou een farmaco-epidemiologische afdeling zijn zonder een goede datamanager? Vele malen was je bereid mij de helpende hand te bieden als ik weer eens met een verzoek voor data kwam of vastzat met het programmeren. Jouw inzet met betrekking tot de Doetinchem cohort is zeer waardevol voor mij.

Svetlana Belitser, beste Svetlana, op het moment dat ik, zeer ingewikkelde analyses met meerdere metingen in de tijd, uit moest voeren heb jij mij voorzien van goede statistische adviezen. Dank je wel!

Dr. W.M.M. Verschuren, beste Monique, bedankt dat jij mij het vertrouwen hebt gegeven om een onderzoek uit te voeren met de serum monsters van de Doetinchem cohort. Ook jouw kennis in de epidemiologie was heel erg waardevol voor het opzetten en analyseren van de studie, en het uiteindelijke manuscript (hoofdstuk 4). Bedankt!

Toen ik eenmaal groen licht van de Doetinchem onderzoeksgroep had gekregen, hadden Liset de la Fonteyne, Arja de Klerk, Bhawanie Nagarajah, Henny Verhagen, Bert Verlaan, Jolanda Vermeulen en Ilse Tonk, met alle ontberingen van dien, de monsters uit de vriezers gelicht. Daar ben ik jullie allen dankbaar voor!

Eenmaal met de serum monsters in Maastricht aangekomen, namen Mia Wolfs en Jos Austen mij gelijk onder hen hoede. Beste Mia en Jos, bedankt dat jullie mij wegwijs hebben gemaakt in het laboratorium van de klinische immunologie. Vanaf de eerste dag voelde ik mij daar erg welkom. Verder wil ik ook José Barron-Houben, Yvon Knapen-Portz, Jolanda Knops-Janssen, Carla Langejans, Kathleen Mallet, Joyce Niessen, Dionne van Opbergen, Marie-Paule Paulissen en Claire Wieczorek bedanken voor alle hulp die ik van jullie heb gekregen bij het bepalen van de (auto) immune markers. Met mijn beperkte ervaring in het laboratorium heb ik ontzettend veel van jullie allen geleerd. Ook was ik onder de indruk van jullie gastvrijheid. Bedaank!

Beste Eric Gremmer, ik wil jou in het bijzonder bedanken voor de grote rol die je hebt gespeeld in het bepalen van de (auto) immuun markers uit het serum van de deelnemers uit de Doetinchem cohort. Elke week bleef je één nacht over in Maastricht om zo vol gas zoveel mogelijk monsters door te meten. Maar na gedane arbeid is het goed rusten, en daarom wil ik jou ook bedanken voor de gezellige avonden in Maastricht. Beste Eugéne Jansen, bedankt dat je van alle monsters het CRP gehalte hebt bepaald.

Dr. J.G.M.C. Damoiseaux, beste Jan, al ben ik een 'groentje' in de immunologie, graag wil ik jou bedanken voor jouw immunologische input, de brainstorm sessies en jouw feedback op het manuscript (hoofdstuk 4).

Dank aan dr. M.P. Hoevenaar-Blom, de datamanagers A. Blokstra, J. van der Laan en RJ. de Klein en alle andere medewerkers van de Doetinchem cohort (ook de veldmedewerkers van de GGD). Beste Marieke, Anneke, Jan en Robert Jan, ook al werkten 
jullie aan vele projecten, er was altijd wel een gaatje in jullie drukke agenda's te vinden. Bedankt voor jullie ondersteuning in het verstrekken van de data, programmeren en de statistische suggesties, wat heeft bijgedragen aan de resultaten die zijn beschreven in hoofdstuk 4 van dit proefschrift.

Prof. dr. W. Slob, beste Wout, ook al was ik niet direct betrokken bij het proefdieronderzoek, jouw raad en daad met betrekking tot het programma 'PROAST' waren zeer waardevol en droegen bij aan het resultaat uit hoofdstuk 5.

De leden van de beoordelingscommissie, prof. dr. H. ten Cate, prof. dr. W. van den Berg, prof. dr. A.C.G. Egberts, prof. dr. J. Kleinjans, en prof. dr. P.W. de Leeuw ben ik zeer erkentelijk voor het doornemen van mijn proefschrift.

De vormgeving van dit proefschrift was in handen van Harald Pieper. Beste Harald, met jouw precisie en creatieve inbreng is het een mooi proefschrift geworden. Bedankt! Furthermore, I would like to thank Annette Spedding, Annette Herzog and Elaine Kingwell for editing my thesis. Your English is superb!

Als je al enige tijd in het buitenland vertoeft, merk je toch dat het Nederlands iets minder goed uit de verf komt. Tessa Dillerop, bedankt voor het doornemen van de Nederlandse samenvatting.

Tijdens mijn promotie heb ik op vier verschillende afdelingen gewerkt. Dank aan alle dames (Addy, Ineke, Suzanne en Anja van Farmacoepidemiologie, Universiteit Utrecht; Nanette en Anita van Klinische en Experimentele Immunologie, Universiteit Maastricht; Willy en Janet van het laboratorium voor gezondheidsbeschermend onderzoek (GBO), RIVM; en Marie Claire, Sandrien en Christa van Toxicogenomics, Universiteit Maastricht) van de secretariaten voor de ondersteuning en belangstelling voor mijn promotieonderzoek.

Al mijn collega's van de afdeling farmaco-epidemiologie, bedankt voor jullie ondersteuning, gezelligheid, de vrijdagnamiddag borrels, en de gezamenlijke lunches en dineetjes. Simone, Mira, Hans, Jacqueline, Renate, Miriam en Suzanne, ik vond het erg prettig jullie allen als kamergenoten te hebben gehad. Bedankt voor de gezelligheid! Mijn speciale dank gaat uit naar Ellen Koster. Ik heb jou niet alleen leren kennen als een fijne collega, maar in de loop der jaren is onze samenwerking uitgegroeid tot een bijzondere vriendschap. Bedankt dat ik met jou kon sparren over een aantal epidemiologische vraagstukken, je hulp in het programmeren en alle gezellige uitjes. Ik had je graag aan mijn zijde op de promotie gehad, maar op het moment dat de magische woorden 'hora est' worden uitgesproken, ben jij op rondreis in Australië. Heel veel plezier daar! 
Naast mijn collega's van de afdeling farmaco-epidemiologie wil ik ook de collega's (waaronder mijn kamergenoten Marten en Maria) van Toxicogenomics bedanken voor jullie gastvrijheid, warmte en behulpzaamheid. Thank you, Christina, Daneida, Maria, Marieke and Marlon for enjoying my company after work. In het bijzonder wil ik Anneloes Melman bedanken. Niet alleen was je er voor me om allerlei (administratieve) klusjes voor me af te handelen, maar je stond ook altijd voor me klaar. Dit heb ik zeer gewaardeerd! Daarnaast wil ik jou bedanken voor je gastvrijheid en alle leuke uitjes. Fijn dat je tijdens de promotie als paranimf naast me staat!

Ook wil ik de collega's van GBO onderzoek bedanken. Bedankt voor de prettige samenwerking en de gezamenlijke koffiebreaks en lunches. Margriet, Annemieke, Marianne en Erna, bedankt dat ik een tijdje jullie kamergenote mocht zijn. Al was het maar kort, bedankt voor jullie gezelligheid!

Na mijn officiële promotietraject ben ik aan de slag gegaan bij het NIVEL. Ik dank mijn voormalige collega's voor het sparren over epidemiologische vraagstukken en het programmeren in SAS. Furthermore, I would like to thank Christel, Susanne and Esther for having me as your roommate. Thank you very much for the dinners and drinks after work.

Currently, I am working at the department of Neurology, UBC. I would like to thank all my colleagues for providing me with epidemiological information for the manuscript as presented in chapter 3.2, and supporting me in the last phase of my PhD.

Prof. dr. Hoepelman en dr. Oldenburg, beste Andy en Bas, bedankt voor jullie zorg, de belangstelling in het onderzoek en de tomeloze inzet bij de oversteek naar Canada.

Naast promoveren is er natuurlijk meer in het leven. Uiteraard wil ik al mijn vrienden bedanken die het gehele promotietraject op een afstandje hebben gevolgd. Lieve Aline, Annette, Chantal, Daniëlle, Elly, Emilie, Liesbeth, Mayke, Marloes Janknegt, Marloes van Zwam, Mieke, Renate, Rianne, Rosanne, Sietske, en Tessa, ondanks de vele verhuizingen, emigraties en de gezinnetjes stichten, wil ik jullie allen danken voor de jarenlange vriendschappen. Bedankt voor jullie interesse in mijn onderzoek, de gezellige (nonsens) gesprekken, de etentjes en borrels, en natuurlijk de weekendjes weg. Ik vind het fijn dat jullie er altijd voor me zijn!

Lieve voedingsmiepjes, Eunice, Esther en Hilde, in al die jaren hebben jullie regelmatig voor de nodige ontspanning gezorgd. Bedankt voor jullie belangstelling, warmte, hilarische gesprekken, culinaire hoogstandjes en natuurlijk de niet te vergeten culturele uitjes. 
Lieve Sander, Angelique, Bart, Judith, Tom, Maaike, Geerten, Harriëtte, Carolien, Jan, dank voor jullie jarenlange vriendschap! Ik hoop dat we onze tradities van kroegavondjes, Cultus-inn avonden en bizarre verjaardagen in Dronten nog heel lang in stand kunnen houden! Ook lieve heer Giesen, heer Krol, Mark, Christian, Christina, Jantina, Sunita en Yvonne hebben voor de nodige afleiding gezorgd. Bedankt voor de gezellige dagjes/avondjes!

Jaarclub Zotz, oftewel, lieve Chantal, Geke, Jolanda, Lenny en Renske bedankt voor alle leuke uitstapjes, het kokkerellen van heerlijke maaltijden en de interessante gesprekken.

Dear Kingston friends (Chi, Claire, Rhonda and Sonya), after all these years you still support me in all the things I do. Thank you!

In het bijzonder wil ik Manon bedanken. Lieve Manon, sinds de middelbare school lopen we al samen op. Al die jaren heb je mij op de voet gevolgd. Bedankt voor je betrokkenheid, je 'pep talks' als ik het even niet zag zitten, je enthousiasme en inspiratie, de hilarische gesprekken, en alle leuke etentjes, borrels en dagjes weg. Ik vind het heel fijn dat jij mijn paranimf wilt zijn!

En natuurlijk wil ik ook mijn 'kleine' familie bedanken. Lieve Gerlof, Marie, Diana, Johan, Clara, Siebrand en Tineke, bedankt voor jullie interesse in het promotieonderzoek. Fijn dat jullie er op de belangrijke momenten voor me zijn!

Janet, lief zusje, Arne en Janaika bedankt voor jullie belangstelling in het onderzoek en de afleidingsmanoeuvres. Succes met de laatste loodjes van de zwangerschap! Hanna, mijn lieve zusje en huisgenote, afgelopen jaren waren voor ons beide, jaren van buffelen. Jij met je opleiding tot register-accountant en ik met het promotieonderzoek. Binnenkort gaan we ook op jouw afronding toosten! Lieve Hanna, bedankt voor je begrip als ik weer eens in de stress was, je luisterend oor en het bijhouden van de papierwinkel met betrekking tot ons huisje. Pas je nog een beetje goed op Huize 'Jantje van der Heijden'?

Lieve pap en mam, jullie hebben me altijd onvoorwaardelijk gesteund en aangemoedigd in alle keuzes die ik heb gemaakt. Dank je wel voor alle liefde en vertrouwen die jullie me in het leven hebben gegeven!

Allen bedankt!

Hilda 


\section{LIST OF PUBLICATIONS}

\section{PAPERS RELATED TO THE THESIS}

De Jong HJ, Cohen Tervaert JW, Saldi SR, Vandebriel RJ, Souverein PC, Meyboom $\mathrm{RH}$, Van Loveren $\mathrm{H}$, Klungel $\mathrm{OH}$. Association between statin use and lupus-like syndrome using spontaneous reports. Semin Arthritis Rheum. 2011; 41(3):373-81.

De Jong HJ, Saldi SR, Klungel OH Vandebriel RJ, Souverein PC, Meyboom RH, Passier JL, Van Loveren H, Cohen Tervaert JW. Statin-associated polymyalgia. An analysis using WHO global individual case safety database: a case/non-case approach. PLoS One. 2012; 7(7):e41289.

De Jong HJ, Meyboom RH, Klungel OH, Niskanen L, Cohen Tervaert JW. Giant cell arteritis/ polymyalgia rheumatica after re-exposure with a statin. Ann Intern Med. 2014; 161(8):614-5.

De Jong HJ, Klungel OH, Van Dijk L, Vandebriel RJ, Leufkens HG, Van der Laan JW, Cohen Tervaert JW, Van Loveren H. Use of statins is associated with an increased risk of rheumatoid arthritis. Ann Rheum Dis. 2012; 71(5):648-54.

De Jong HJ, Cohen Tervaert JW, Lalmohamed A, De Vries, F, Vandebriel RJ, Van Loveren $\mathrm{H}$, Klungel $\mathrm{OH}$, Van Staa TP. Pattern of risks of Rheumatoid Arthritis among patients using statins: a cohort study with the Clinical Practice Research Datalink. (submitted)

De Jong HJ, Vandebriel RJ, Saldi SR, Van Dijk L, Van Loveren H, Cohen Tervaert JW, Klungel $\mathrm{OH}$. Angiotensin-converting enzyme inhibitors or angiontensin II receptor blockers and the risk of developing rheumatoid arthritis in antihypertensive drug users. Pharmacoepidemiol Drug Saf. 2012; 21(8):835-43.

De Jong HJ, Damoiseaux JG, Vandebriel RJ, Souverein PC, Gremmer E, Wolfs M, Klungel OH, Van Loveren H, Cohen Tervaert JW, Verschuren WM. Statin use and markers of (auto) immunity in the Doetinchem Cohort Study. PLoS One. 2013; 8(10):e77587.

Vandebriel RJ, De Jong HJ, Gremmer ER, Klungel OH, Cohen Tervaert JW, Slob W, Van Der Laan JW, Van Loveren $\mathrm{H}$. Statins accelerate the onset of collagen type II-induced arthritis in mice. Arthritis Res Ther. 2012; 14(2):R90.

Vandebriel RJ, De Jong HJ, Gremmer ER, Klungel OH, Tervaert JW, Slob W, Van Der Laan JW, Van Loveren $\mathrm{H}$. Response to 'Statins accelerate the onset of collagen type II-induced arthritis in mice'-authors' reply. Arthritis Res Ther. 2013; 15(2): 403

De Jong HJ, Van Staa TP, Lalmohamed A, Vandebriel RJ, Van Loveren H, Klungel $\mathrm{OH}$, Cohen Tervaert JW. Pattern of risks of Systemic Lupus Erythematosus among statin users: a population-based study in the United Kingdom. (in preparation) 


\section{PAPERS NOT RELATED TO THE THESIS}

De Jong HJ, Korevaar JC, Van Dijk L, Voogd E, Van Dijk CE, Van Oijen MG. Suboptimal prescribing of proton pump inhibitors in low-dose aspirin users in general practice: a population based cohort study. BMJ Open. 2013; 3(7): doi:pii: e003044.

Van Dijk L, De Jong HJ, Voogd E, Van Dijk CE, Van Oijen MG, Korevaar JC. Discontinuation of Proton Pump Inhibitors in users of Low-Dose Aspirin: A cohort study in general practice. (submitted)

De Jong HJ, Korevaar JC, Voogd E, Van Dijk CE, Van Oijen MG, Van Dijk L. Incidence, prevalence and trends of low-dose aspirin and concomitant use of proton pump inhibitors in general practitioner patients, 2001 to 2010. (in preparation)

De Jong HJ, De Goede J, Oude Griep LM, Geleijnse JM. Alcohol consumption and blood lipids in elderly coronary patients. Metabolism. 2008; 57(9):1286-92.

\section{ABSTRACTS}

\section{RELATED TO THIS THESIS}

De Jong H, Saldi S, Souverein P, Meyboom R, Vandebriel R, Cohen Tervaert JW, Van Loveren $\mathrm{H}$, Klungel $\mathrm{O}$. Association between the use of statins and systemic lupus erythematosus. Drug Saf. 2010; 33(10):907.

Saldi S, De Jong H, Souverein P, Meyboom R, Vandebriel R, Cohen Tervaert JW, Van Loveren $\mathrm{H}$, Klungel $\mathrm{O}$. Association between the use of statins and systemic lupus erythematosus. Pharmacoepidemiol Drug Saf. 2010; 19(suppl1):S148.

De Jong H, Saldi S, Klungel O, Vandebriel R, Souverein P, Meyboom R, Van Loveren $\mathrm{H}$, Cohen Tervaert JW. The association between statin use and polymyalgia rheumatic/arteritis temporalis: demonstrated by spontaneous reports and self-described case-reports. Pharmacoepidemiol Drug Saf. 2011;20(suppl1):S68.

De Jong H, Klungel O, Van Dijk L, Vandebriel R, Leufkens H, Cohen Tervaert JW, Van Loveren $\mathrm{H}$. Use of statins and the risk of rheumatoid arthritis. Pharmacoepidemiol Drug Saf. 2009; 18(suppl1):S126.

De Jong HJ, Cohen Tervaert JW, Lalmohamed A, De Vries F, Vandebriel RJ, Van Loveren $\mathrm{H}$, Klungel $\mathrm{OH}$, Van Staa T. Pattern of risks of rheumatoid arthritis among patients using statins: a cohort study with the Clinical Practice Research Datalink. Pharmacoepidemiol Drug Saf. 2014; 23(suppl1):S2. 
De Jong H, Vandebriel R, Saldi S, Van Dijk L, Van Loveren H, Cohen Tervaert JW, Klungel $O$. Angiotensin-converting enzyme inhibitors or angiotensin II receptor blockers and the risk of developing rheumatoid arthritis in antihypertensive drug users. Pharmacoepidemiol Drug Saf. 2011; 20(suppl1):S67.

De Jong H, Cohen Tervaert JW, Klungel O, Souverein P, Vandebriel R, Damoiseaux $\mathrm{J}$, Van Loveren $\mathrm{H}$, Verschuren M. Statin use and the prevalence of autoantibodies in the Doetinchem cohort. Pharmacoepidemiol Drug Saf. 2010; 19(suppl1):S146.

\section{NOT RELATED TO THIS THESIS}

De Jong HJ, Korevaar JC, Van Dijk L, Voogd E, Van Dijk CE, Van Oijen MG. Suboptimal prescribing of proton pump inhibitors in low-dose aspirin users in general practice. Pharmacoepidemiol Drug Saf. 2013; 22(suppl1):S184

De Jong H, Van Dijk L, Voogd E, Korevaar J, Van Oijen M. Redefining gastroprotection in low-dose aspirin users: intentional gastroprotection is very low in general practice. Gut. 2012; 61(suppl3):A92.

De Jong H, De Goede J, Geleijnse JM. Alcohol consumption is inversely associated with high density lipoprotein cholesterol (HDLC) in coronary patients. Eur J Epid. 2006: 21(suppl1):95 


\section{LIST OF CO-AUTHORS PRESENTED IN THIS THESIS}

\section{Jan Willem Cohen Tervaert}

Internal Medicine and Immunology, Maastricht University Medical Centre, Maastricht, the Netherlands

Sint Franciscus Gasthuis, Rotterdam, the Netherlands

\section{Jan G.M.C. Damoiseaux}

Central Diagnostic Laboratory, Maastricht University Medical Centre, Maastricht, the Netherlands

\section{Liset van Dijk}

Netherlands Institute for Health Services Research (NIVEL), Utrecht, the Netherlands

\section{Eric R. Gremmer}

Centre for Health Protection, National Institute for Public Health and the Environment (RIVM), Bilthoven, the Netherlands

\section{Markku J. Helle}

Department of Pathology, Mikkeli Central Hospital, Mikkeli, Finland

\section{Olaf H. Klungel}

Division of Pharmacoepidemiology and Clinical Pharmacology, Department of Pharmaceutical Sciences, Faculty of Sciences, Utrecht Institute for Pharmaceutical Sciences, Utrecht University, Utrecht, the Netherlands

\section{Jan-Willem van der Laan}

Section on Pharmacology, Toxicology and Biotechnology, Medicines Evaluation Board, Utrecht, the Netherlands

\section{Arief Lalmohamed}

Department of Clinical Pharmacy, University Medical Centre Utrecht, Utrecht, the Netherlands

Division of Pharmacoepidemiology and Clinical Pharmacology, Department of Pharmaceutical Sciences, Faculty of Sciences, Utrecht Institute for Pharmaceutical Sciences, Utrecht University, Utrecht, the Netherlands

\section{H.G.M. (Bert) Leufkens}

Division of Pharmacoepidemiology and Clinical Pharmacology, Department of Pharmaceutical Sciences, Faculty of Sciences, Utrecht Institute for Pharmaceutical Sciences, Utrecht University, Utrecht, the Netherlands

Medicines Evaluation Board, Utrecht, the Netherlands 


\section{Henk van Loveren}

Centre for Health Protection, National Institute for Public Health and the Environment (RIVM), Bilthoven, the Netherlands

Department of Toxicogenomics, Maastricht University Medical Centre, Maastricht, the Netherlands

\section{Ronald H.B. Meyboom}

Division of Pharmacoepidemiology and Clinical Pharmacology, Department of Pharmaceutical Sciences, Faculty of Sciences, Utrecht Institute for Pharmaceutical Sciences, Utrecht University, Utrecht, the Netherlands

Uppsala Monitoring Centre, World Health Organisation Collaborating Centre for International Drug Monitoring, Uppsala, Sweden

\section{Leo Niskanen}

Pharmacovigilance, Finnish Medicines Agency Fimea, Helsinki, Finland

\section{J.L.M. (Anneke) Passier}

Netherlands Pharmacovigilance Centre Lareb,'s-Hertogenbosch, the Netherlands

\section{Siti R.F. Saldi}

Centre for Clinical Epidemiology and Evidence-Based Medicine, Cipto Mangunkusumo Hospital \& Faculty of Medicine University of Indonesia, Jakarta, Indonesia

\section{Wouter Slob}

Centre for Substances and Integrated Risk Assessment, National Institute for Public Health and the Environment (RIVM), Bilthoven, the Netherlands

\section{Patrick C. Souverein}

Division of Pharmacoepidemiology and Clinical Pharmacology, Department of Pharmaceutical Sciences, Faculty of Sciences, Utrecht Institute for Pharmaceutical Sciences, Utrecht University, Utrecht, the Netherlands

\section{Tjeerd P. van Staa}

Health eResearch Centre, Farr Institute for Health Informatics Research, University of Manchester, Manchester, United Kingdom

Department of non-communicable diseases, London School of Hygiene \& Tropical Medicine, London, United Kingdom

Division of Pharmacoepidemiology and Clinical Pharmacology, Department of Pharmaceutical Sciences, Faculty of Sciences, Utrecht Institute for Pharmaceutical Sciences, Utrecht University, Utrecht, the Netherlands 


\section{Rob J. Vandebriel}

Centre for Health Protection, National Institute for Public Health and the Environment (RIVM), Bilthoven, the Netherlands

\section{W.M. Monique Verschuren}

Centre for Nutrition, Prevention and Health Services, National Institute for Public Health and the Environment (RIVM), Bilthoven, the Netherlands

\section{Frank de Vries}

Division of Pharmacoepidemiology and Clinical Pharmacology, Department of Pharmaceutical Sciences, Faculty of Sciences, Utrecht Institute for Pharmaceutical Sciences, Utrecht University, Utrecht, the Netherlands

School for Public Health and Primary Care (CAPHRI), Maastricht University Medical Centre, Maastricht, the Netherlands

Department of Clinical Pharmacy and Toxicology, Maastricht University Medical Centre, Maastricht, the Netherlands

MRC Epidemiology Lifecourse Unit, Southampton General Hospital, Southampton, United Kingdom

\section{Mia Wolfs}

Central Diagnostic Laboratory, Maastricht University Medical Centre, Maastricht, the Netherlands 


\section{ABOUT THE AUTHOR}

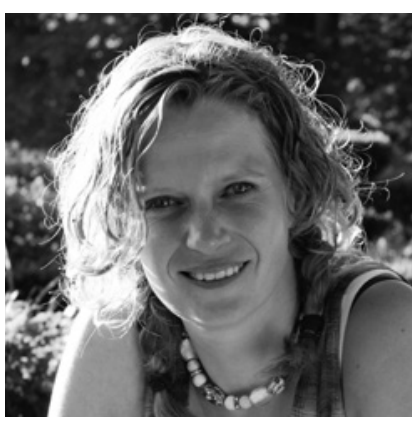

Hilda de Jong was born on July 13th, 1976 in Dronten, the Netherlands. In 1995, she completed secondary school at the Johannes Calvijn Lyceum in Kampen, the Netherlands. Afterwards, she started her study of Environmental Sciences at the Hogeschool IJselland in Deventer where she obtained her Bachelor's degree (BSc) in 1999. In the same year she started her study in Human Nutrition and Health at the Wageningen University, the Netherlands. During her study she completed a research traineeship at the Centre for

Primary Care Studies, Queen's University, Kingston, Canada, and her MSc thesis was entitled: "Alcohol consumption and blood lipids in elderly coronary patients". After obtaining her MSc degree in Human Nutrition (major in epidemiology) in 2006, she started working as a nutritionist at Unilever where she became interested in clinical research. With this specific interest in mind, she successfully gained a place in a joint PhD program in 2007 at the Maastricht University Medical Centre, Utrecht University and the National Institute for Public Health and the Environment (RIVM), the Netherlands. Her PhD research focused on the association between cardiovascular drugs and the risk of developing systemic autoimmune diseases, as has been described in this thesis. In 2011, she started working at NIVEL (Netherlands Institute for Health Services Research) where she conducted studies in the prescribing regimes of proton pump inhibitors in patients receiving low-dose aspirin in general practice. Subsequently, NIVEL offered her the possibility of pursuing her dream of becoming an international researcher. With this international collaboration, she started working as a postdoctoral research fellow at the division of Neurology, University of British Columbia (UBC), Vancouver, Canada, in September 2012. During her work, she was awarded two postdoctoral research fellowships from the MS society of Canada (2013-2015) and the Michael Smith Foundation for Health Research (2013-2016) to study: "New indications of old drugs: Do statins, angiotensin converting enzyme inhibitors or proton pump inhibitors impact long-term disease progression in multiple sclerosis?". Furthermore, she has been selected for the 2014-2015 EndMS Scholar Program for Researchers IN Training (SPRINT) from the EndMS Research and Training Network and MS Society of Canada. Currently, she is a postdoctoral research fellow at UBC and the school of Mental Health and Neuroscience, Maastricht University Medical Centre. 


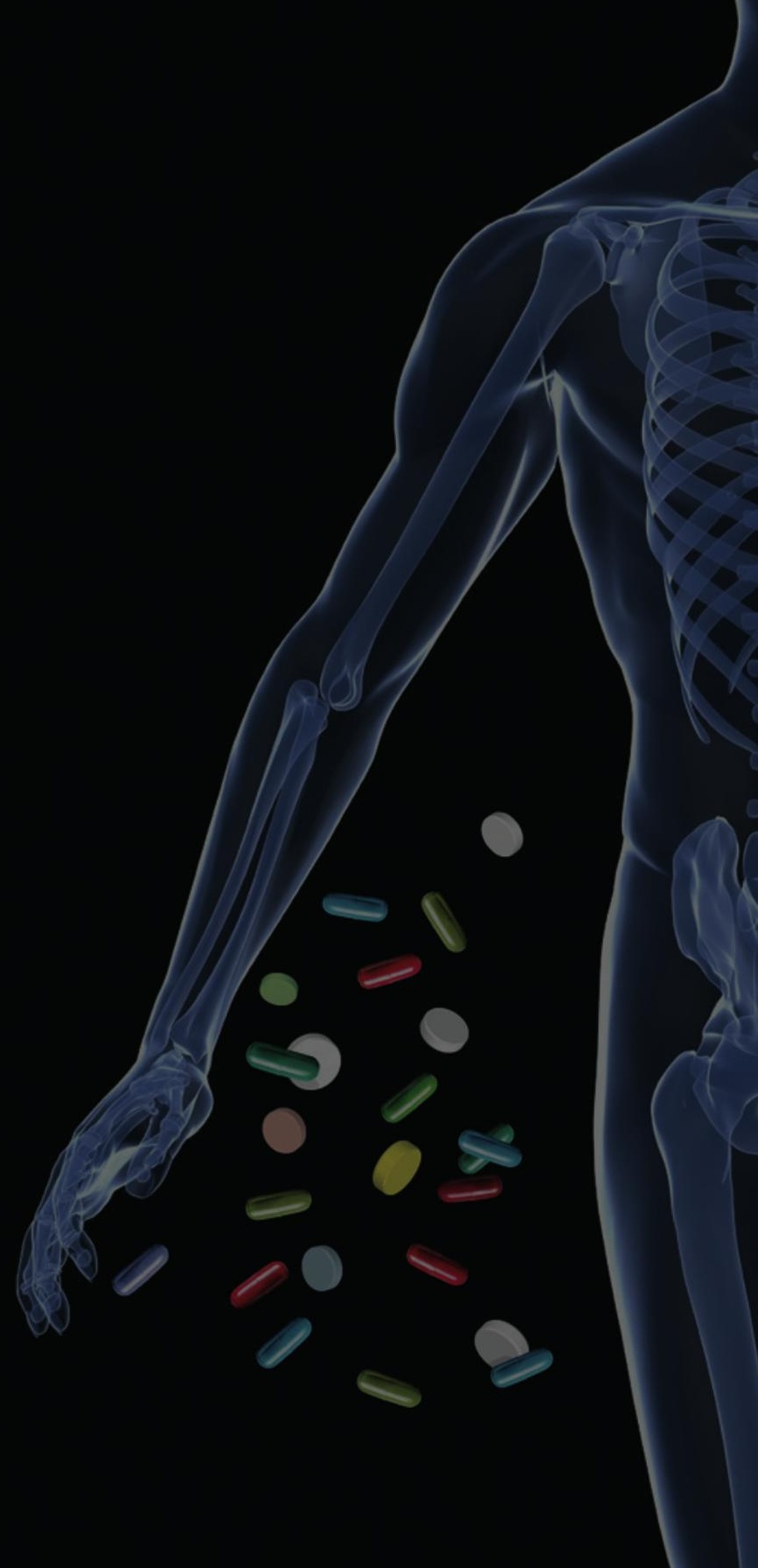

